\title{
Multiplicação escalar eficiente em curvas elípticas
}

\author{
Adão de Melo Neto
}

\author{
DISSERTAÇÃO APRESENTADA \\ AO \\ INSTITUTO DE MATEMÁTICA E ESTATÍSTICA \\ DA \\ UNIVERSIDADE DE SÃO PAULO \\ PARA \\ OBTENÇÃO DO TÍTULO DE MESTRE \\ EM \\ CIÊNCIAS
}

Área de Concentração: Ciência da Computação

Orientador: Prof. Dr. Routo Terada

São Paulo, dezembro de 2006 


\section{Multiplicação escalar eficiente em curvas elípticas}

Este exemplar corresponde à redação final da dissertação devidamente corrigida e defendida por Adão de Melo Neto e aprovada pela comissão Julgadora.

São Paulo, 02 de janeiro de 2007.

Banca Examinadora:

Prof. Dr. Routo Terada (orientador) - IME/USP

Profa. Dra. Liria Matsumoto Sato - EP/USP

Prof. Dr. Paulo Sérgio Licciardi Messeder Barreto - EP/USP 
Para minha esposa Marieta e minha filha Patrícia Lívia que compreenderam minha dedicação e abdicaram da minha atenção integral, em prol do desenvolvimento desta dissertação. 


\section{Agradecimentos}

A Deus, que me deu vida e saúde, permitindo que eu concluísse este trabalho.

A meu pai Jair, por seu espírito combativo e pelo seu amor ao trabalho e a minha mãe Ivone, pelo seu espírito de sacrifício e por seu imenso amor a todos os seus filhos. Vocês são um exemplo para todos nós.

A minha querida esposa Marieta e a minha filha Patrícia, que compreenderam a minha dedicação e abdicaram de minha atenção integral, em prol do desenvolvimento desta dissertação.

A meu orientador, Routo Terada, pelo tempo precioso que dedicou à minha orientação.

Ao professor Paulo Sérgio Licciardi Messeder Barreto, por suas preciosas dicas ao longo desse trabalho.

A professora Liria Matsumoto Sato, por sua colaboração.

A todos os professores e funcionários do Instituto de Matemática e Estatística.

Enfim, a todos aqueles que contribuiram para este momento.

O meu Deus, segundo as Suas riquezas, suprirá todas as vossas necessidades em glória, por Cristo Jesus.

(Paulo aos Filipenses, Cap. IV, versículo 19) 


\section{Resumo}

Em 1985, Koblitz e Miller propuseram independentemente usar o grupo de pontos sobre uma curva elíptica definida sobre um corpo finito em criptosistemas baseados no problema do logaritmo discreto. A vantagem principal que criptosistemas sobre curvas elípticas tem sobre os tradicionais criptosistemas baseados no problema do logaritmo discreto em um grupo multiplicativo de um corpo finito (e também sobre os criptosistemas baseados na intratabilidade da fatoração inteira) é o desconhecimento de um algoritmo de tempo subexponencial que poderia encontrar logaritmos discretos no grupo elíptico. Outra vantagem é o fato dos protocolos padrões em criptografia que fazem o uso do problema do logaritmo discreto no grupo multiplicativo de um corpo finito, tal como o protocolo Diffie-Hellman e os protocolos ElGamal, serem adaptáveis para curvas elípticas. Contudo, a multiplicação escalar de pontos da curva elíptica é mais custosa do que a operação equivalente daquele grupo. Este trabalho estuda os principais algoritmos que aceleram essa operação em curvas elípticas não-supersingulares definidas sobre corpos de característica igual a 2 (corpos binários) e de característica maior do que 3 (corpos primos). Note-se que os algoritmos não consideram possíveis otimizações nas operações aritméticas básicas. 


\begin{abstract}
In 1985, Koblitz and Miller independently proposed using the group of points on an elliptic curve defined over a finite field in discrete logarithm cryptosystems. The main advantage that elliptic curve cryptosystems have over cryptosystems based on the multiplicative group of a finite field (and also over cryptosystems based on the intractability of integer factorization) is the lack of a subexponencial-time algorithm that could find discrete logarithms in the elliptic group. Another advantage is the fact that the standard cryptographic protocols that use the discrete logarithm problem on the multiplicative group of a finite field, like the Diffie-Hellman and ElGamal protocols, are adaptable to elliptic curves. However, the scalar multiplication in elliptic curves is more expensive than the equivalent operation in that group. This work studies the main algorithms that speed up this operation in non-supersingular elliptic curves defined over finite fields of characteristic equal to 2 (binary fields) and of characteristic bigger than 3 (prime fields). Observe that possible optimizations of the basic arithmetic operations are not considered in the algorithms.
\end{abstract}




\section{Sumário}

Lista de Figuras $\quad$ xi

Lista de Tabelas $\quad$ xii

Notação $\quad$ xiv

1 Introdução 1

1.1. Organizaçào do documento . . . . . . . . . . . . 1

2 Curvas elípticas 3

2.1 Histórico . . . . . . . . . . . . . . . . 3

2.2 Problema do logaritmo discreto em curvas elípticas (PLDCE) 4

2.3 Aritnética em curvas elípticas . . . . . . . . . . . 4

2.4 Curvas elípticas isomorfas . . . . . . . . . . . 5

2.5 Equação de Weierstrass simplificada . . . . . . . . . . . 5

2.6 Lei de grupo . . . . . . . . . . . . . . . . . . . . 6

2.6 .1 Método geométrico . . . . . . . . . . . 6

2.6 .2 Método algébrico . . . . . . . . . . . . 6

2.7 Coordenadas projetivas . . . . . . . . . . . . 10

2.7.1 Curva elíptica $y^{2}=x^{3}+a x+b \ldots \ldots \ldots 11$

2.7.2 Curva elíptica $y^{2}+x y=x^{3}+a x^{2}+b \ldots \ldots 12$

2.8 Padrões . . . . . . . . . . . . . . . . . 14

2.9 Aspectos relativos a Segurança . . . . . . . . . . . 15

2.10 Conclusão . . . . . . . . . . . . . . . . . 16

3 Métodos gerais de multiplicação... 17

3.1 Cadeias aditivas . . . . . . . . . . . . . . . 17

3.2 Sequèncias aditivas . . . . . . . . . . . . . . 18

3.3 Nétodo binário . . . . . . . . . . . . . . . . . . . 19

3.4 Método s-ário . . . . . . . . . . . . . . 20

3.5 Método janela deslizante . . . . . . . . . . . . . . . 21

3.6 Método BGMW . . . . . . . . . . . . . . . . . . . 21

3.7 Adaptação dos métodos para curvas elípticas . . . . . . . . . 23

3.7 .1 Cadeias de adição e subtração . . . . . . . . . 23 
3.7 .2 Forna não-adjacente (NAF) . . . . . . . . 23

3.7.3 Método binário . . . . . . . . . . . . . 25

3.7 .4 Método NAF binário . . . . . . . . . . . . . . . 26

3.7.5 Forma não-adjacente de dimensão $w \ldots \ldots$. . . . . 26

3.7 .6 Método $2^{w}$-ário NAF . . . . . . . . . . . . . . . . 27

3.7 .7 Método janela deslizante . . . . . . . . . . 28

3.7 .8 Método BGMW . . . . . . . . . . . . . . . . 29

3.8 Conclusão . . . . . . . . . . . . . . . . . . . . . . . . 29

4 Curvas de Koblitz elípticas com boas propriedades criptográ-

ficas 31

4.1 Endomorfismo de Frobenius . . . . . . . . . . . . . . . 31

4.2 Multiplicação complexa . . . . . . . . . . . . . 31

4.3 Curva anômala . . . . . . . . . . . . . . . . 32

4.4 Multiplicação em curvas elípticas anômalas de característica 232

4.4 Números de Pontos . . . . . . . . . . . . . . . . 33

4.4.2 Exemplos definidos em $\mathbb{E}_{2} \ldots \ldots \ldots \ldots . \ldots 34$

4.4 .3 Exemplos definidos en $\mathbb{F}_{4} \ldots \ldots \ldots \ldots$

4.4.4 Exemplos definidos en $\mathbb{F}_{8} \ldots \ldots \ldots . \ldots . \ldots 35$

4.4.5 Exemplos definidos em $\mathbb{F}_{16} \ldots \ldots \ldots \ldots$

4.4 .6 Conchusăo . . . . . . . . . . . . 36

4.5 Alguns aspectos da implementação eficiente . . . . . . . . 36

4.5 .1 Expansão binária balanceada . . . . . . . . . 36

4.5.2 Domínio Euclidiano e função norma . . . . . . . 37

4.5 .3 Expansăo na hase $\varphi \ldots \ldots \ldots . \ldots . \ldots 37$

4.5.4 Expansão balanceada na base $\bullet . \ldots \ldots \ldots . \ldots 37$

4.6 Conclusão . . . . . . . . . . . . . . . . . . . . . . . 38

5 Método de Meier e Staffelbach para multiplicação em curvas definidas sobre $\mathbb{F}_{2} \quad 39$

5.1 Algoritmo para computar a expansão balanceada na base $\alpha \quad 45$

5.2 Conclusào . . . . . . . . . . . . . . . . . 45

6 Método de Solinas para multiplicação em curvas definidas $\begin{array}{ll}\text { sobre } \mathbb{F}_{2} & 47\end{array}$

6.1 Multiplicação escalar elíptica . . . . . . . . . . . 47

6.2 Expansão NAF $\tau$-ádica . . . . . . . . . . . . . . . . . 49

6.3 Divisão e redução modular em $\mathbb{Z}[\tau] \ldots \ldots . . \ldots . . .52$

6.4 Algoritmo de multiplicação . . . . . . . . . . . . . 54

6.5 Conclusão . . . . . . . . . . . . . . . . . 57

7 Método de Volker Müller $\quad 58$

7.1 Representando inteiros cono una soma de potências de $\varphi$. . 59

7.2 Multiplicarsão de pontos usando a expansão de Frobenius . . . 62 
7.3 Custo do algoritmo . . . . . . . . . . . . . . . . . . 64

7.4 Conclusão . . . . . . . . . . . . . . . 65

8 Forma Esparsa Conjunta de Solinas $\quad 66$

8.1 Métodos de Shamir . . . . . . . . . . . . . . 66

8.1.1 Método trivial . . . . . . . . . . . 67

8.1.2 Método simples de Shamir . . . . . . . . . . 67

8.1.3 Método rápido de Shamir . . . . . . . . . . . 67

8.2 Expansões binárias com sinal . . . . . . . . . . 68

8.3 Forma Esparsa Conjunta (FEC) . . . . . . . . . . . . . . 69

8.4 Computando a Forma Esparsa Conjunta . . . . . . . . . 72

8.5 Corretude do algoritmo . . . . . . . . . . 76

8.5.1 Corretude das regras de transição entre estados . . . 81

8.6 Otimalidade da FEC . . . . . . . . . . . . 83

8.7 Eficiéncia do método rápido de Shamir usando a FEC . . . 86

8.7 .1 Comprimento da FEC . . . . . . . . . . . . 86

8.8 Conclusão . . . . . . . . . . . . . . . . . . . 88

9 Método GLV $\quad 91$

9.1 Endomorfismos . . . . . . . . . . . . . . . . . . . . 91

9.2 A técnica empregada . . . . . . . . . . . . . . . 93

9.2 .1 Linhas gerais . . . . . . . . . . . . . . . . . 93

9.2 .2 Decomposição de $K$. . . . . . . . . . . . . . . 95

9.3 Conclusão . . . . . . . . . . . . . . . . . . . . . . . . 98

10 Métodos de Ciet, Lange, Sica e Quisquater 99

10.1 Extensão da expansão T-ádica das curvas de Koblitz para ceertas curvas definidas sobre corpos primos com endomorfismo eficiente . . . . . . . . . . . . . . . . . . . . 99 99

10.1.1 Decomposição chave: expansão $\phi \ldots . . . . . .100$

10.1.2 Expansão $\phi$-ádica de densidade $1 / 3 \ldots \ldots$. . . . 104

10.1.3 Comparação con o método NAF binário . . . . . . . 105

10.1.4 Comparaçấo con o método GLV . . . . . . . 105

10.2 Forma $\phi$-Esparsa Conjunta . . . . . . . . . . . . 105

10.3 Aplicações da $\phi$-FEC . . . . . . . . . . . . . . . . . 113

10.4 Comparações: endomorfismo de Frobenius . . . . . . . 114

10.5 Comparações: endomorfismo da curva do exemplo 10.1 . . . 114

10.6 Obtenção da $\phi$-FEC na curva do exemplo $10.2 \ldots \ldots . .715$

10.7 Conclusão . . . . . . . . . . . . . . . . . 116

11 Conclusões $\quad 118$

1.1.1. Multiplicação simples . . . . . . . . . . . . . . . . 119

11.1 .1 Métodos gerais de multiplicação . . . . . . . . . . 119

11.1.2 Multiplicação em curvas binárias anomalas . . . . 122 
11.1.3 Multiplicação em curvas definidas em pequenos corpos de característica $2 \ldots \ldots \ldots$. . . . . . . . . 122

11.1.4 Multiplicação em curvas definidas sobre corpos primos 123

11.2 Multiplicaçâo dupla . . . . . . . . . . . . . . . . 125

11.2.1 Multiplicação dupla en curvas binárias anômalas . . . 125

11.2.2 Multiplicação dupla em curvas definidas sobre corpos primos . . . . . . . . . . . . . . . 125

Apêndice A: Conceitos básicos $\quad 127$

3 Notação $O(n)$ e o(n) . . . . . . . . . . . . 127

4 Problema do logaritmo discreto . . . . . . . . . . . 127

5 Protocolo de troca de chaves Diffie-Helman . . . . . . . . . 128

6 Aritmética modular . . . . . . . . . . . . . . . 128

7 Pequeno teorema de Fermat . . . . . . . . . . . . . . . 128

8 Grupo . . . . . . . . . . . . . . . . . . . . . . 129

9 Anel . . . . . . . . . . . . . . . . . . . . . . . . . . . . . 129

10 Corpo . . . . . . . . . . . . . . . . . . 130

11 Corpo primo . . . . . . . . . . . . . . . . . . 130

12 Corpo binário . . . . . . . . . . . . . . . . . . 131

13 Corpo estendido . . . . . . . . . . . . . . . . . . 131

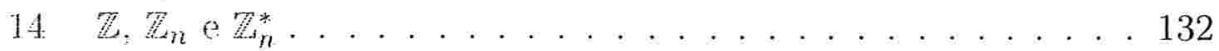

15 Bases normais de $\mathbb{F}_{2^{2 n}} \ldots \ldots \ldots \ldots \ldots \ldots \ldots$

16 Homomorfismo . . . . . . . . . . . . . . . 133

17 Endomorfismo . . . . . . . . . . . . . . . . 133

18 Relaçầo de equivalência . . . . . . . . . . . . 133

Apêndice B: Implementação 134

Referências bibliográficas 172 


\section{Lista de Figuras}

2.1. Soma de pontos en una curva elíptica $(P+Q=R) \ldots 6$

5.1 O reticulado $\mathbb{Z}[\alpha] \ldots \ldots \ldots \ldots \ldots \ldots \ldots$

5.2 Casos não-crítico, semi-crítico e crítico. . . . . . . . 42

8.1 Fluxo da transição entre os 8 estados do Algoritmo 8.2 . . . 80 


\section{Lista de Tabelas}

2.1 Contagen das operações de adição e duplicação de pontos em

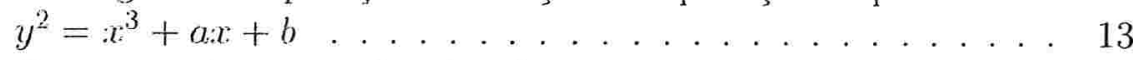

2.2 Contagem das operações de adição e duplicação de pontos em $y^{2}+x y=x^{3}+a x^{2}+b \ldots \ldots \ldots \ldots 14$

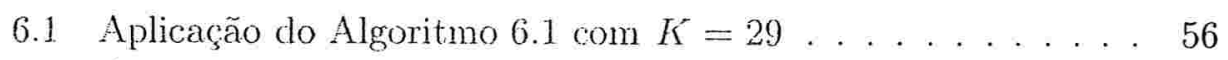

6.2 Algoritmo $6.3 \mathrm{com} a=1, x=9$ e $y=0 \ldots \ldots . \ldots 56$

8.2 Transição entre estados . . . . . . . . . . . . . . . 79

11.1 Métodos gerais de multiplicação . . . . . . . . . . . . . . 121

11.2 Multiplicação em curvas binárias anòmalas . . . . . . . . . 122

11.3 Multiplicação em curvas definidas em pequenos corpos de característica $2 \ldots \ldots \ldots \ldots \ldots \ldots$

11.4 Multiplicação em curvas definidas sobre corpos primos . . . . 124

11.5 Multiplicação dupla em curvas binárias anômalas . . . . . . 125

11.6 Multiplicação dupla em curvas definidas sobre corpos primos. 126 


\section{Notação}

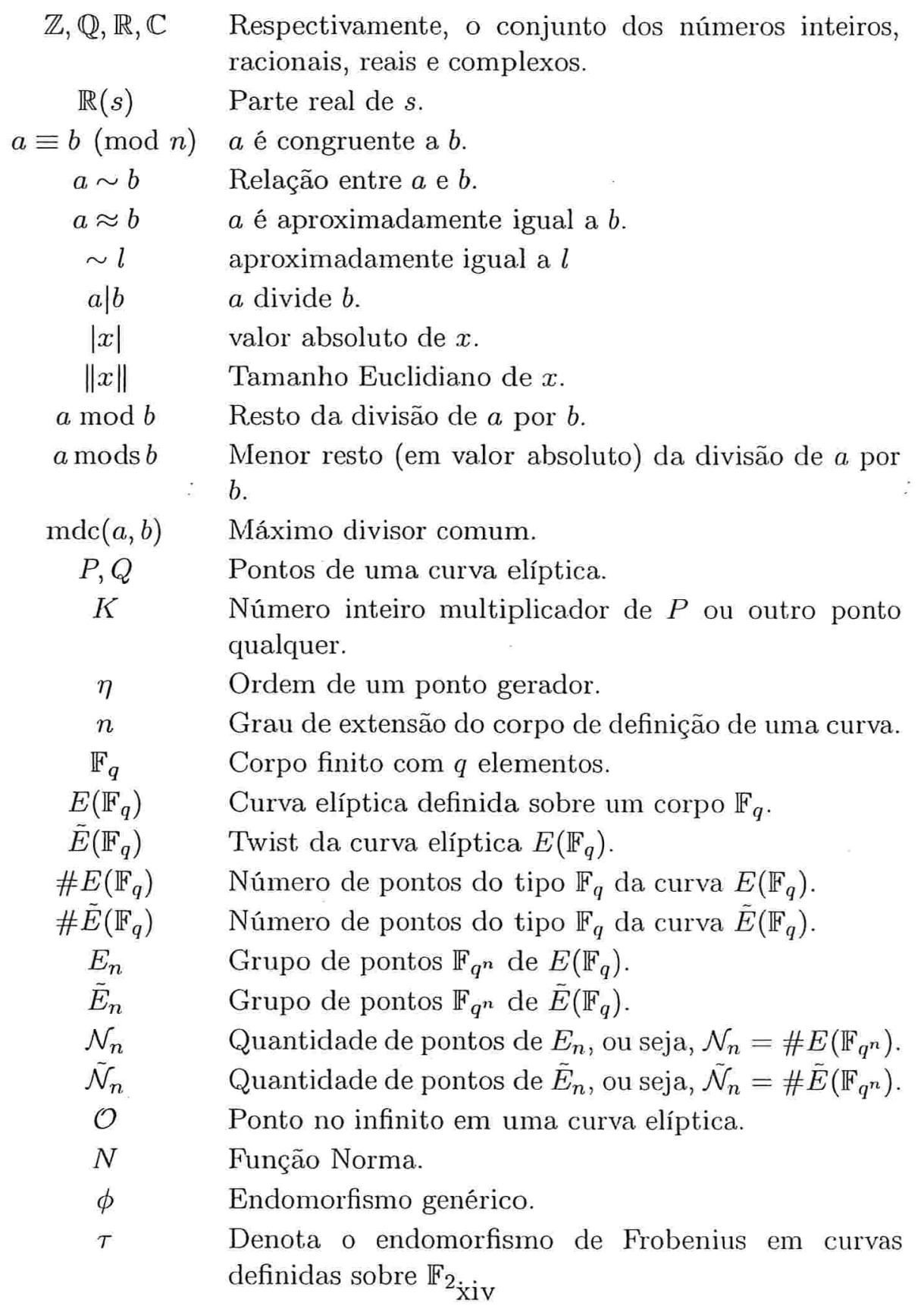


$\varphi \quad$ Endomorfismo de Frobenius.

$\alpha \quad$ Número contido no conjunto dos números complexos que é a solução da equação característica de um endomorfismo $\phi$. Muitos autores abusam na notação ao usar a notação $\phi$ em substituição a $\alpha$ em seus artigos.

$\bar{\alpha} \quad$ Conjugado de $\alpha$. Por exemplo, se $\alpha=a-b i(a, b \in \mathbb{Z})$, então $\bar{\alpha}=a-b i$.

$\operatorname{End}(E) \quad$ Anel endomórfico de $E$.

$\mathbb{Z}[\phi]$ Anel formado pelo conjunto de polinômios $a+b \alpha$, onde $a, b \in \mathbb{Z}$.

$\langle P\rangle \quad$ Grupo cíclico constituído por todos os produtos finitos de $P$.

$c \quad$ Traço do endomorfismo de Frobenius.

$\mathcal{K} \quad$ Corpo genérico.

$\mathcal{L} \quad$ Corpo estendido genérico.

NAF Non-Adjacent Form.

GLV Gallant-Lambert-Vanstone.

FEC Forma Esparsa Conjunta.

$\phi$-FEC Forma $\phi$-Esparsa Conjunta. 


\section{Capítulo 1}

\section{Introdução}

O objetivo do nosso trabalho é estudar os métodos de multiplicação de pontos em curvas elípticas culminando com a implementação das propostas que foram sugeridas em [3] para acelerar a multiplicação simples $K P$, bem como a multiplicação dupla $K_{0} P+K_{1} Q$, onde $K_{0}, K_{1}, K \in \mathbb{Z}$ e $P$ e $Q$ são pontos de uma curva elíptica.

\subsection{Organização do documento}

No capítulo 2 são apresentados conceitos fundamentais referentes a curvas elípticas.

No capítulo 3 são apresentados métodos de multiplicação em curvas elípticas que não fazem uso de qualquer característica particular da curva e portanto são aplicados a quaisquer curvas. São métodos derivados dos métodos de exponenciação em grupos multiplicativos [7].

No capítulo 4 é apresentado o trabalho de Koblitz [10] que propõe curvas elípticas com boas propriedades criptograficas. Este trabalho trata basicamente das curvas anômalas definidas sobre pequenos corpos de característica 2 (os corpos $\mathbb{F}_{2}, \mathbb{F}_{4}, \mathbb{F}_{8}, \mathbb{F}_{16}$ ). $\mathrm{O}$ autor propõe o uso do endomorfismo de Frobenius para a aceleração da multiplicação nas referidas curvas.

Nos capítulos 5 e 6 são apresentados métodos para multiplicação em curvas elípticas definidas em $\mathbb{F}_{2}$ (curvas binárias anômalas). São os métodos de Meier e Staffelbach [12], no capítulo 5 e o de Solinas [2]] no capítulo 6.

No capítulo 7 é apresentado o método de Volker Müller [18] para multiplicação em curvas elípticas definidas sobre pequenos corpos de característica 2 (os corpos $\mathbb{F}_{4}, \mathbb{F}_{8}, \mathbb{F}_{16}$ ).

No capítulo 8 é apresentada a Forma Esparsa Conjunta (FEC) de Solinas [22], uma expansão binária conjunta de dois inteiros positivos $K_{0}$ e $K_{1}$ que possui peso de Haming conjunto mínimo, o que permite a computação eficiente da multiplicação dupla $K_{0} P+K_{1} Q$ que é utilizado na verificação de assinaturas digitais bem como na computação de $K P$ utilizando o método 
GLV descrito no capítulo 9.

No capítulo 9 é apresentado o método Gallant-Lambert-Vanstone (GLV) para multiplicação em curvas elípticas definidas sobre corpos primos que possuam endomorfismos computáveis eficientemente.

O capítulo 10 descreve duas propostas. A primeira propõe uma técnica para estender a expansão NAF $\tau$-ádica das curvas de Koblitz [2]], como descrito no capítulo 6 para uma classe de curvas definidas sobre corpos primos que possuem endomorfismos computáveis eficientemente. Na realidade o método é aplicado em dois exemplos de curvas definidas sobre corpos primos, uma cujo endomorfismo satisfaz o polinômio mínimo $\phi^{2}-\phi+2=0$ e outra que satisfaz ao polinômio mínimo $\phi^{2}+2=0$. A segunda proposta é a Forma $\phi$-Esparsa Conjunta ( $\phi$-FEC), uma expansão conjunta na base $\phi$ de dois inteiros positivos $K_{0}$ e $K_{1}$ de peso de Haming conjunto mínimo, onde $\phi$ é um endomorfismo que possui polinômio mínimo na forma $\phi^{2}-r \phi+2=0$, onde $r \in\{0, \pm 1\}$. A $\phi$-FEC permite acelerar a computação de $K_{0} P+K_{1} Q$ bem como a computação de $K P$, tanto no caso das curvas definidas sobre $\mathbb{F}_{2}$, quanto nos dois exemplos de curvas citados anteriormente.

O capítulo 11 apresenta nossas conclusões.

No Apéndice A são descritos alguns conceitos básicos. O Apêndice B contém o código-fonte da implementação. 


\section{Capítulo 2}

\section{Curvas elípticas}

Todas as formas conhecidas de criptografia assimétrica baseiam-se em algum problema matemático de certa maneira intratável (mais precisamente, NP-completo), sendo assim natural classificar os algoritmos assimétricos de acordo com o problema matemático em que se baseiam.

Ao contrário do que ocorre com algoritmos simétricos, há poucas classes conhecidas de algoritmos assimétricos que tenham resistido a tentativas exaustivas de criptoanálise. Dentre essas classes, as de maior sucesso são a dos algoritmos baseados no problema da fatoração inteira e a dos algoritmos baseados no problema do logaritmo discreto. O algoritmo RSA é um exemplo da primeira classe. Exemplos da segunda classe são os sistemas criptográficos de curvas elípticas, considerados hoje o estado da arte em criptografia assimétrica.

\subsection{Histórico}

O trabalho pioneiro de Whitfield Diffie e Martin Hellman sobre criptografia assimétrica incluía um algoritmo para distribuição pública de chaves baseado na dificuldade de calcular logaritmos discretos sobre o grupo $\mathbb{Z}_{p}^{*}$ (ver Apêncice). Na época, os melhores algoritmos conhecidos para o cálculo desses logaritmos tinham complexidade $\mathcal{O}(\sqrt{p})$, ou seja, exponencial no número de bits de $p$.

Não decorreu muito tempo até que algoritmos mais eficientes fossem encontrados, em particular o algoritmo de cálculo de índices e variantes, que, embora ainda sejam inviáveis com parâmetros adequados do sistema criptográfico, possuem complexidade subexponencial, implicando um aumento considerável no tamanho das chaves empregadas e, em conseqüência, nos tempos de processamento do protocolo originais de Diffie e Hellman e derivados.

Contudo, o mesmo protocolo pode ser implementado sobre outros grupos além do $\mathbb{Z}_{p}^{*}$. A descoberta de algoritmos mais eficientes para o problema do logaritmo discreto inspirou a busca de grupos onde esse problema fosse mais 
difícil. O primeiro resultado promissor nessa direção foi encontrado em 1985 , independentemente por dois pesquisadores, Neil Koblitz e Victor Miller, e reside nas entidades matemáticas conhecidas como curvas elípticas.

\subsection{Problema do logaritmo discreto em curvas elíp- ticas (PLDCE)}

O PLDCE é: dado uma curva elíptica $E$ definida sobre um corpo finito $\mathbb{F}_{q}$, um ponto $P \in E\left(\mathbb{F}_{q}\right)$ de ordem $\eta$, e um ponto $Q \in\langle P\rangle$, encontrar o inteiro $K \in[0, \eta-1]$ tal que $Q=K P$. O inteiro $K$ é chamado o logaritmo discreto de $Q$ na base $P$, e é denotado $K=\log _{P} Q$.

\subsection{Aritmética em curvas elípticas}

Uma curva elíptica $E$ sobre um corpo $\mathcal{K}$ é definida pela equação

$$
E: y^{2}+a_{1} x y+a_{3} y=x^{3}+a_{2} x^{2}+a_{4} x+a_{6} . \quad \text { (Equação de Weierstrass) }
$$

onde $a_{1}, a_{2}, a_{3}, a_{4}, a_{6} \in \mathcal{K}$ e $\Delta \neq 0$, onde $\Delta$ é o discriminante de $E$ e é definido como se segue:

$$
\begin{aligned}
& \Delta=-d_{2}^{2} d_{8}-8 d_{4}^{3}-27 d_{6}^{2}+9 d_{2} d_{4} d_{6} \\
& d_{2}=a_{1}^{2}+4 a_{2} \\
& d_{4}=2 a_{4}+a_{1} a_{3} \\
& d_{6}=a_{3}^{2}+4 a_{6} \\
& d_{8}=a_{1}^{2} a_{6}+4 a_{2} a_{6}-a_{1} a_{3} a_{4}+a_{2} a_{3}^{2}+a_{4}^{3} .
\end{aligned}
$$

Se $\mathcal{L}$ é um corpo estendido de $\mathcal{K}$, então o conjunto de pontos $\mathcal{L}$ sobre $E$ é

$$
\begin{aligned}
E(\mathcal{L}):\left\{(x, y) \in \mathcal{L} \times \mathcal{L}: y^{2}+a_{1} x y+a_{3} y\right. & = \\
& \left.x^{3}+a_{2} x^{2}+a_{4} x+a_{6}\right\} \cup\{\mathcal{O}\} .
\end{aligned}
$$

onde $\mathcal{O}$ é o ponto no infinito.

\section{Observaçōes:}

1. Dizemos que $E$ é definida sobre o corpo $\mathcal{K}$ (corpo base) porque os coeficientes da equação que define $E$ são elementos de $\mathcal{K}$. Escrevemos $E(\mathcal{K})$ para enfatizar que $E$ é definida sobre $\mathcal{K}$.

2. A condição $\Delta \neq 0$ garante que cada ponto da curva possui uma e somente uma linha tangente. 
3. Os pontos $\mathcal{L}$ sobre $E$ são os pontos $(x, y)$ que satisfazem a equação da curva cujas coordenadas $x$ e $y$ pertencem a $\mathcal{L}$. O ponto $\mathcal{O}$ é considerado um ponto de $\mathcal{L}$ para todos os corpos estendidos $\mathcal{L}$ de $\mathcal{K}$.

\subsection{Curvas elípticas isomorfas}

Duas curvas elípticas $E_{1}$ e $E_{2}$ definidas sobre $\mathcal{K}$ e dadas pelas equações

$$
E_{1}: y^{2}+a_{1} x y+a_{3} y=x^{3}+a_{2} x^{2}+a_{4} x+a_{6}
$$

e

$$
E_{2}: y^{2}+b_{1} x y+b_{3} y=x^{3}+b_{2} x^{2}+b_{4} x+b_{6} .
$$

são isomorfas sobre $\mathcal{K}$ se existe $u, r, s, t \in \mathcal{K}(u \neq 0)$, tal que a troca de variáveis

$$
(x, y) \rightarrow\left(u^{2} x+r, u^{3} y+u^{2} s x+t\right)
$$

transformam a equação $E_{1}$ na equação $E_{2}$. A referida transformação é chamada uma troca admissível de variáveis.

\subsection{Equação de Weierstrass simplificada}

A Equação de Weierstrass, $E: y^{2}+a_{1} x y+a_{3} y=x^{3}+a_{2} x^{2}+a_{4} x+a_{6}$, definida sobre $\mathcal{K}$, pode ser simplificada considerando a aplicação de trocas admissiveis de variáveis. Iremos considerar dois casos: a característica de $\mathcal{K}$ é diferente de 2 e de 3 e a característica de $\mathcal{K}$ é igual a 2 . Nos dois casos que serão apresentados as curvas são não-supersingulares.

Curva definida sobre corpo de característica diferente de 2 ou 3:

$\mathcal{K}=\mathbb{F}_{q}$, onde $q=p$ e $p>3$ é um número primo. Nesse caso, a troca admissível de variáveis

$$
(x, y) \rightarrow\left(\frac{x-3 a_{1}^{2}-12 a_{2}}{36}, \frac{y-3 a_{1} x}{216}-\frac{a_{1}^{3}+4 a_{1} a_{2}-12 a_{3}}{24}\right)
$$

transforma $E$ na curva

$$
y^{2}=x^{3}+a x+b
$$

onde $a, b \in \mathcal{K}$. O discriminante desta curva é $\Delta=-16\left(4 a^{3}+27 b^{2}\right)$.

Curva definida sobre corpo de característica 2

$\mathcal{K}=\mathbb{F}_{q}$ e $q=2^{n}$ e $n \geq 1$. Se $a_{1} \neq 0$, então a troca admissível de variáveis

$$
(x, y) \rightarrow\left(a_{1}^{2} x+\left(\frac{a_{3}}{a_{1}}\right), a_{1}^{3} y+\left(\frac{a_{1}^{2} a_{4}+a_{3}^{2}}{a_{1}^{3}}\right)\right)
$$

transforma $E$ na curva

$$
y^{2}+x y=x^{3}+a x^{2}+b
$$

onde $a, b \in \mathcal{K}$. O discriminante desta curva é $\Delta=b$. 


\subsection{Lei de grupo}

Seja $E$ uma curva elíptica definida sobre $\mathcal{K}$. Existe uma regra para soma de dois pontos em $E$ para se obter um terceiro ponto em $E$. Junto com esta operação de adição, o conjunto de pontos $E$ forma um grupo abeliano com $\mathcal{O}$ servindo como elemento identidade. Este é o grupo que é usado na construção de sistemas criptográficos baseados em curvas elípticas.

\subsubsection{Método geométrico}

Para calcularmos geometricamente a soma dos pontos $P$ e $Q$, primeiramente, traçamos uma reta entre $P$ e $Q$, interceptando a curva em um terceiro ponto, que chamaremos de $-R$. O resultado de $P+Q$ é o ponto simétrico de $-R$ em relação ao eixo das abscissas, ou seja, $P+Q=R$.

Na Figura 2.1 podemos ver um exemplo de como calcular geometricamente a soma de pontos na curva elíptica $y^{2}=x^{3}-7 x+5$. Consideramos que os pontos da curva são números reais.

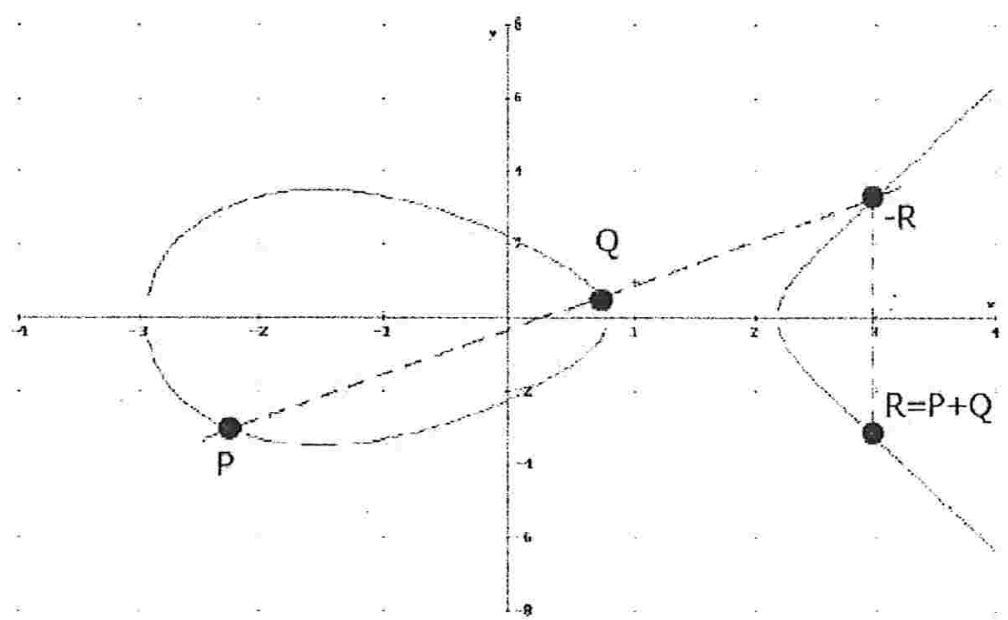

Figura 2.1: Soma de pontos em uma curva elíptica $(P+Q=R)$.

Para duplicarmos geometricamente um $P$, tomamos uma tangente da curva no ponto $P$. Tal linha intercepta a curva em um terceiro ponto, que chamaremos de $-R$. O resultado de $P+P$ é o ponto simétrico de $-R$ em relação ao eixo das abscissas, ou seja, $2 P=R$.

\subsubsection{Método algébrico}

Curva elíptica $E(\mathcal{K}): y^{2}=x^{3}+a x+b$

$\mathcal{K}=\mathbb{F}_{q}$, onde $q=p$ e $p>3$ é um número primo. 
- Identidade:

$P+\mathcal{O}=\mathcal{O}+P$ para todos os $P \in E(\mathcal{K}) ;$

- Negação:

Seja $P=(x, y)$. Então, $-P=(x,-y)$;

- Adição de pontos:

$x_{3}=t^{2}-x_{1}-x_{2}$ e $y_{3}=t\left(x_{1}-x_{3}\right)-y_{1}$, onde $t=\left(y_{2}-y_{1}\right) /\left(x_{2}-x_{1}\right)$;

- Duplicação:

$x_{3}=t^{2}-2 x_{1}$ e $y_{3}=t\left(x_{1}-x_{3}\right)-y_{1}$, onde $t=\left(3 x_{1}^{2}+a\right) /\left(2 y_{1}\right)$.

Curva elíptica $E(\mathcal{K}): y^{2}+x y=x^{3}+a x^{2}+b$

$\mathcal{K}=\mathbb{F}_{q}, q=2^{n}$ e $n \geq 1$.

- Identidade:

$P+\mathcal{O}=\mathcal{O}+P$ para todos os $P \in E(K) ;$

- Negação:

Seja $P=(x, y)$. Então, $-P=(x, x+y)$;

- Adição de pontos:

$x_{3}=\lambda^{2}+\lambda+x_{1}+x_{2}+a$ e $y_{3}=\lambda\left(x_{1}+x_{3}\right)+x_{3}+y_{1}$, onde $\lambda=\left(y_{2}+y_{1}\right) /\left(x_{2}+x_{1}\right)$;

- Duplicação de pontos:

$x_{3}=\lambda^{2}+\lambda+x_{1}+x_{2}+a$ e $y_{3}=\lambda\left(x_{1}+x_{3}\right)+x_{3}+y_{1}$, onde $y=\left(x_{1}^{2}+y_{1}\right) /\left(x_{1}\right)$.

Exemplo 2.1 (Curva elíptica $E\left(\mathbb{F}_{p}\right): y^{2}=x^{3}+a x+b$ onde $p>3$ ). Considere a curva elíptica $E: y^{2}=x^{3}+2 x+4$ definida sobre $\mathbb{F}_{11}$. Note que $\Delta=-16\left(4 a^{3}+27 b^{2}\right) \not \equiv-7424(\bmod 11) \neq 0$, tal que $E$ é de fato uma curva elíptica.

Determinando os pontos sobre $E$ :

Primeiro deve-se verificar para quais possíveis valores de $y \in \mathbb{F}_{11}$, o valor $z$ é um resíduo quadrático. Os valores de $z$ são $1,3,4,5,9$, pois

\begin{tabular}{c|ccccccccccc}
\hline$y$ & 0 & 1 & 2 & 3 & 4 & 5 & 6 & 7 & 8 & 9 & 10 \\
\hline$z=y^{2}(\bmod 11)$ & 0 & 1 & 4 & 9 & 5 & 3 & 3 & 5 & 9 & 4 & 1 \\
\hline
\end{tabular}

Como $p+1$ é divisível por 4 , se $z$ é um resíduo quadrático, é fácil calcular as raízes quadradas de $z$. De fato $\pm z^{(11+1) / 4}(\bmod 11)= \pm z^{3}(\bmod 11)$ são as duas raízes quadradas de $z$ [25], pois $\left(z^{(p+1) / 4}\right)^{2} \equiv z^{(p+1) / 2} \equiv z^{(p-1) / 2} \cdot z \equiv$ $z(\bmod p)$ pelo Teorema de Fermat (ver Apendice). 


\begin{tabular}{c|c|c}
\hline$x$ & $z=\left(x^{3}+2 x+4\right)(\bmod 11)$ & $y= \pm z^{3}(\bmod 11)$ \\
\hline 0 & 4 & 9 e 2 \\
1 & 7 & - \\
2 & 5 & 4 e 7 \\
3 & 4 & 9 e 2 \\
4 & 10 & - \\
5 & 7 & - \\
6 & 1 & e 10 \\
7 & 9 & 3 e 8 \\
8 & 4 & 9 e 2 \\
9 & 3 & 5 e 6 \\
10 & 1 & 1 e 10 \\
\hline
\end{tabular}

A segunda coluna representa o valor de $x^{3}+2 x+4$ reduzido ao módulo 11 , ou seja, o resto da divisão de $x^{3}+2 x+4$ por 11 e a terceira coluna representa o valor de $y$ tal que $y^{2}(\bmod 11)$ dá como resultado o valor correspondente na segunda coluna. Deste modo, temos que:

Se $y=9$, então $y^{2}(\bmod 11)=81(\bmod 11)=4$.

Se $y=2$, então $y^{2}(\bmod 11)=4(\bmod 11)=4$.

Se $y=4$, então $y^{2}(\bmod 11)=16(\bmod 11)=5$.

Se $y=7$, então $y^{2}(\bmod 11)=49(\bmod 11)=5$.

Se $y=1$, então $y^{2}(\bmod 11)=1(\bmod 11)=1$.

Se $y=10$, então $y^{2}(\bmod 11)=100(\bmod 11)=1$.

Se $y=3$, então $y^{2}(\bmod 11)=9(\bmod 11)=9$.

Se $y=8$, então $y^{2}(\bmod 11)=64(\bmod 11)=9$.

Se $y=5$, então $y^{2}(\bmod 11)=25(\bmod 11)=3$.

Se $y=6$, então $y^{2}(\bmod 11)=36(\bmod 11)=3$.

Os demais valores de $y^{2}$ não possuem um correspondente $y$ tal que $y^{2}$ ( $\bmod 11)$ dê como resultado o valor na segunda coluna da tabela.

Deste modo, a curva elíptica $E\left(\mathbb{F}_{11}\right): y^{2}=x^{3}+2 x+4$ tem 17 pontos, que são: $(0,9),(0,2),(7,3),(7,8),(2,4),(2,7),(8,9),(8,2),(3,9),(3,2)$, $(9,5),(9,6),(6,1),(6,10),(10,1),(10,10)$ e o ponto $\mathcal{O}$.

Temos um total de 17 pontos, isto é, a ordem do grupo é 17. Qualquer grupo de ordem prima é cíclico, logo os pontos de $E$ são isomórfos a $Z_{17}$. Ademais, qualquer ponto de $E$, distinto de $\mathcal{O}$ é o gerador dos pontos de $E$.

Vamos agora utilizar as equações do método algébrico, para calcular o ponto $R=P+Q$, sendo $P=(7,3)$ e $Q=(2,4)$ pontos da curva $E\left(\mathbb{F}_{11}\right)$ : $y^{2}=x^{3}+2 x+4$.

$$
\begin{aligned}
t & =\frac{y_{2}-y_{1}}{x_{2}-x_{1}} \equiv \frac{4-3}{2-7} \equiv-\frac{1}{5} \equiv-\frac{45}{5} \equiv-9 \quad(\bmod 11)=2 . \\
x_{3} & =t^{2}-x_{1}-x_{2} \equiv 4-9 \equiv-5 \quad(\bmod 11)=6 . \\
y_{3} & =t\left(x_{1}-x_{3}\right)-y_{1} \equiv 2(7-6)-3 \equiv-1 \quad(\bmod 11)=10 .
\end{aligned}
$$


Vamos agora utilizar as equações do método algébrico, para calcular o ponto $S=2 P$, sendo $P=(7,3)$ curva $E\left(\mathbb{F}_{11}\right): y^{2}=x^{3}+2 x+4$.

$$
\begin{aligned}
t & =\frac{3 x_{1}^{2}+a}{2 y_{1}} \equiv \frac{3 x_{1}^{2}+a}{2 y_{1}} \equiv \frac{149}{6} \equiv \frac{6}{6} \equiv 1 \quad(\bmod 11)=1 . \\
x_{3} & =t^{2}-2 x_{1} \equiv 1-14 \equiv-13 \quad(\bmod 11)=9 . \\
y_{3} & =t\left(x_{1}-x_{3}\right)-y_{1} \equiv 1(7-9)-3 \equiv-5 \quad(\bmod 11)=6 .
\end{aligned}
$$

Se quiséssemos, agora, calcular o ponto $3 P$, bastaria somarmos o ponto $S=2 P$ ao ponto $P$ que obteríamos o resultado desejado.

Calculando o ponto $T=3 P=S+P$, ou seja, $(9,6)+(7,3)$.

$$
\begin{aligned}
t & =\frac{y_{2}-y_{1}}{x_{2}-x_{1}} \equiv \frac{3-6}{7-9} \equiv \frac{3}{2} \equiv \frac{14}{2} \equiv 7 \quad(\bmod 11)=7 . \\
x_{3} & =t^{2}-x_{1}-x_{2} \equiv 49-9-7 \equiv 33 \quad(\bmod 11)=0 . \\
y_{3} & =t\left(x_{1}-x_{3}\right)-y_{1} \equiv 7(9-0)-6 \equiv 57 \quad(\bmod 11)=2 .
\end{aligned}
$$

Prosseguindo, analogamente, podemos calcular todos os múltiplos do ponto $P$.

Exemplo 2.2 (Curva $E\left(\mathbb{F}_{2^{n}}\right): y^{2}+x y=x^{3}+a x^{2}+b$, com $n \geq 1$ ). Considere o corpo finito $\mathbb{F}_{2^{4}}$ como que representado pelo polinômio redutor $F(z)=z^{4}+z+1$. Um elemento $a_{3} z^{3}+a_{2} z^{2}+a_{1} z+a_{0} \in \mathbb{F}_{2^{4}}$ é representado pela string de bits $a_{3} a_{2} a_{1} a_{0}$ de comprimento 4 ; por exemplo, (0101) representa $z^{2}+1$. Tomemos $a=z^{3}$ e $b=z^{3}+1$, e considere a curva elíptica $E: y^{2}+x y=x^{3}+z^{3} x^{2}+\left(z^{3}+1\right)$ definida sobre $\mathbb{F}_{2^{4}}$. Os pontos de $E\left(\mathbb{F}_{2^{4}}\right)$ são os seguintes:

$$
\begin{array}{cccc}
\mathcal{O} & (0011,1100) & (1000,0001) & (1100,0000) \\
(0000,1011) & (0011,1111) & (1000,1001) & (1100,1100) \\
(0001,0000) & (0101,0000) & (1001,0110) & (1111,0100) \\
(0001,0001) & (0101,0101) & (1001,1111) & (1111,1011) \\
(0010,1101) & (0111,1011) & (1011,0010) & \\
(0010,1111) & (0111,1100) & (1011,1001) &
\end{array}
$$

Vamos agora utilizar as equações do método algébrico, para cacular o ponto $R=P+Q$, sendo $P=(0001,0001)$ e $Q=(0010,1101)$ pontos da curva $E: y^{2}+x y=x^{3}+z^{3} x^{2}+\left(z^{3}+1\right)$.

$$
\begin{gathered}
\lambda=\frac{\left(y_{2}+y_{1}\right)}{\left(x_{2}+x_{1}\right)} \equiv \frac{(1100)}{(0011)} \equiv \frac{z^{3}+z^{2}}{z+1} \equiv z^{2}=(0100) . \\
x_{3}=\lambda^{2}+\lambda+x_{1}+x_{2}+a \equiv z^{4}+z^{3}+z^{2}+z+1 \\
\equiv z^{3}+z^{2}=(1100) . \\
y_{3}=\lambda\left(x_{1}+x_{3}\right)+x_{3}+y_{1} \equiv z^{2}\left(1+z^{3}+z^{2}\right)+z^{3}+z^{2}+1 \\
\equiv z^{5}+z^{4}+z^{3}+1 \equiv z^{3}-z^{2} \equiv z^{3}+z^{2}=(1100) .
\end{gathered}
$$


Teorema 2.1 (Teorema de Hasse). Seja $E\left(\mathbb{F}_{q}\right)$ uma curva elíptica definida sobre $\mathbb{F}_{q}$ e $\# E\left(\mathbb{F}_{q}\right)$ o número de pontos de $E\left(\mathbb{F}_{q}\right)$ (ou ordem de $E$ sobre $\mathbb{F}_{q}$ ). Então,

$$
q+1-2 \sqrt{q} \leq \# E\left(\mathbb{F}_{q}\right) \leq q+1+2 \sqrt{q}
$$

O intervalo $(q+1-2 \sqrt{2}, q+1+2 \sqrt{q})$ é conhecido como intervalo de Hasse. Uma formulação alternativa para o Teorema de Hasse é a seguinte: se $E$ é definida sobre $\mathbb{F}_{q}$, então $\# E\left(\mathbb{F}_{q}\right)=q+1-c$, com $|c| \leq 2 \sqrt{q}$, onde $c$ é o de traço de $E$ sobre $\mathbb{F}_{q}$. Desde que $2 \sqrt{q}$ é relativamente pequeno comparado com $q$, nós temos que $\# E\left(\mathbb{F}_{q}\right) \approx q$.

Note que se $E$ é uma curva definida sobre $\mathbb{F}_{q}$, então $E$ é também definida sobre qualquer extensão $\mathbb{F}_{q^{n}}$ de $\mathbb{F}_{q}$.

Teorema 2.2. Seja $E$ uma curva elíptica definida sobre $\mathbb{F}_{q}$, e seja $\# E\left(\mathbb{F}_{q}\right)=$ $q+1-c$. Então o número de pontos da curva $E$, considerada sobre o corpo estendido $\mathbb{F}_{q^{n}}$, é $\mathcal{N}_{n}=q^{n}+1-a_{n}$, para todos os $n \geq 2$, onde $a_{n}$ é a seqüência definida recursivamente por

$$
a_{0}=2, \quad a_{1}=c, \quad \ldots \quad a_{n}=a_{1} a_{n-1}-q a_{n-2}(\text { para } n \geq 2)
$$

Em aplicações criptográficas as operações devem ser executadas preferencialmente em um grupo cíclico de ordem prima (ver seção 2.9). Se $\mathcal{N}_{n}$ é primo, então o grupo cíclico é $E_{n}$. Caso contrário, $\mathcal{N}_{n}=f \eta$, onde $\eta$ é a ordem prima do subgrupo cíclico de $E_{n}$ e $f$ é o cofator. Qualquer ponto $P$ do subgrupo cíclico é um gerador do mesmo. Escolhe-se $P$ tal que a ordem de $P$ seja igual a $\eta$.

Definição 2.1 (curva elíptica não-supersingular). Seja $p$ a característica de $\mathbb{F}_{q}$. Uma curva elíptica $E$ definida sobre $\mathbb{F}_{q}$ é supersingular se $p$ divide $c$, onde $c$ é o traço de Frobenius. Se $p$ não divide $c$, então $E$ é nãosupersingular. Curvas elípticas supersingulares não são adequadas para uso criptográfico.

\subsection{Coordenadas projetivas}

As fórmulas para adição de pontos foram apresentadas em coordenadas afins, tanto para a curva de característica definida sobre um corpo de característica diferente de 2 e $3\left(y^{2}=x^{3}+a x+b\right)$, quanto para a curva definida para um corpo de característica $2\left(y^{2}+x y=x^{3}+a x^{2}+b\right)$. Para ambas as curvas, as fórmulas para adição e duplicação de pontos requerem uma inversão e muitas multiplicações no corpo. Se a inversão no corpo é significamente mais custosa do que a multiplicação, então é vantajoso o uso de coordenadas projetivas.

Seja $\mathcal{K}$ um corpo, e sejam $c$ e $d$ inteiros positivos. Pode-se definir uma relação de equivalência (ver Apenclice) no conjunto $\mathcal{K}^{3} \backslash\{(0,0,0)\}$ de triplas 
diferentes de zero sobre $\mathcal{K}$ por: $\left(X_{1}: Y_{1}: Z_{1}\right) \sim\left(X_{2}: Y_{2}: Z_{2}\right)$ se $X_{1}=\lambda^{c} X_{2}$, $Y_{1}=\lambda^{d} Y_{2}$ e $Z_{1}=\lambda Z_{2}$ para algum $\lambda \in \mathcal{K}^{*}$.

A classe de equivalência contendo $(X, Y, Z) \in \mathcal{K}^{3} \backslash\{(0,0,0)\}$ é

$$
(X: Y: Z)=\left\{\left(\lambda^{c} X, \lambda^{d} Y, \lambda Z\right): \lambda \in \mathcal{K}^{*}\right\} .
$$

$(X: Y: Z)$ é denominado ponto projetivo e $(X, Y, Z)$ é conhecido como o representante de $(X: Y: Z)$. O conjunto de todos os pontos projetivos é denotado $\mathbb{P}(\mathcal{K})$. Note que se $\left(X^{\prime}, Y^{\prime}, Z^{\prime}\right) \in(X: Y: Z)$ então $\left(X^{\prime}: Y^{\prime}: Z^{\prime}\right)=$ $(X: Y: Z)$, isto é, qualquer elemento de uma classe de equivalência pode servir como um representante do ponto projetivo. Em particular, se $Z \neq 0$ então $\left(X / Z^{c}, Y / Z^{d}, 1\right)$ é um representante do ponto projetivo $(X: Y: Z)$, e de fato é o único representante com coordenada $Z=1$.

Portanto temos uma correspondência $1: 1$ entre o conjunto de pontos projetivos

$$
\mathbb{P}(\mathcal{K})^{*}=\{(X: Y: Z): X, Y, Z \in \mathcal{K} ; Z \neq 0\}
$$

e o conjunto de pontos afins

$$
\mathbb{A}(\mathcal{K})=\{(x, y): x, y \in \mathcal{K}\} .
$$

Desta forma obtém-se a forma projetiva da equação de uma curva elíptica, bem como as fórmulas de adição e duplicação através da substituição de $x$ por $\frac{X}{Z^{c}}$ e $y$ por $\frac{Y}{Z^{d}}$.

O conjunto de pontos projetivos

$$
\mathbb{P}(\mathcal{K})^{0}=\{(X: Y: Z): X, Y, Z \in \mathcal{K} ; Z=0\}
$$

é chamado de ponto no infinito desde que não corresponde a qualquer ponto afim.

\subsubsection{Curva elíptica $y^{2}=x^{3}+a x+b$}

Muitos tipos de coordenadas projetivas tem sido propostos. Citaremos duas:

Coordenada projetiva padrão: Nesse caso o ponto projetivo $(X: Y: Z)$ corresponde ao ponto afim $(X / Z, Y / Z)$. A equação projetiva da curva é $Y^{2} Z=X^{3}+a X Z^{2}+b Z^{3}$. O ponto infinito corresponde ao ponto $(0: 1: 0)$, enquanto a negação de $(X: Y: Z)$ é $(X:-Y: Z)$.

Nota:

$$
\begin{aligned}
y^{2} & =x^{3}+a x+b \\
& \Rightarrow Y^{2} / Z^{2}=X^{3} / Z^{3}+a X / Z+b \\
& \Rightarrow Y^{2} Z=X^{3}+a X Z^{2}+b Z^{3} .
\end{aligned}
$$


Coordenada projetiva Jacobiana: Nesse caso o ponto projetivo ( $X$ : $Y: Z$ ) corresponde ao ponto afim $\left(X / Z^{2}, Y / Z^{3}\right)$. A equação projetiva da curva é $Y^{2}=X^{3}+a X Z^{4}+b Z^{6}$. O ponto infinito corresponde ao ponto $(1: 1: 0)$, enquanto a negação de $(X: Y: Z)$ é $(X:-Y: Z)$.

Nota:

$$
\begin{aligned}
y^{2} & =x^{3}+a x+b \\
& \Rightarrow Y^{2} / Z^{6}=X^{3} / Z^{6}+a X / Z^{2}+b \\
& \Rightarrow Y^{2}=X^{3}+a X Z^{4}+b Z^{6} .
\end{aligned}
$$

Exemplo 2.3 (Fórmulas para duplicação em coordenadas Jacobianas). Seja $P=\left(X_{1}: Y_{1}: Z_{1}\right) \in E$ e suponha que $P \neq-P$. Desde que $P=\left(X_{1} / Z_{1}^{2}: Y_{1} / Z_{1}^{3}: 1\right)$, podemos usar a fórmula de duplicação de $E$ em coordenadas afins para computar $2 P=\left(X_{3}^{\prime}: Y_{3}^{\prime}: 1\right)$, obtendo:

$$
\begin{aligned}
t & =\frac{3 x_{1}^{2}+a}{2 y_{1}}=\frac{3\left(X_{1}^{2} / Z_{1}^{4}\right)+a}{2\left(Y_{1} / Z_{1}^{3}\right)}=\frac{\left(3 X_{1}^{2}+a Z_{1}^{4}\right) / Z_{1}^{4}}{2\left(Y_{1} / Z_{1}^{3}\right)}=\frac{3 X_{1}^{2}+a Z_{1}^{4}}{2 Z_{1} Y_{1}} \\
X_{3}^{\prime} & =\left(\frac{3 X_{1}^{2}+a Z_{1}^{4}}{2 Z_{1} Y_{1}}\right)^{2}-2 \frac{X_{1}}{Z_{1}^{2}}=\frac{\left(3 X_{1}^{2}+a Z_{1}^{4}\right)^{2}-8 X_{1}^{2} Y_{1}^{2}}{4 Y_{1}^{2} Z_{1}^{2}} \\
Y_{3}^{\prime} & =\left(\frac{3 X_{1}^{2}+a Z_{1}^{4}}{2 Z_{1} Y_{1}}\right)\left(\frac{X_{1}}{Z_{1}^{2}}-X_{3}^{\prime}\right)-\frac{Y_{1}}{Z_{1}^{3}} .
\end{aligned}
$$

Para eliminar os denominadores na expressão para $X_{3}^{\prime}$ e $Y_{3}^{\prime}$, fazemos $X_{3}=X_{3}^{\prime} Z_{3}^{2}$ e $Y_{3}=Y_{3}^{\prime} Z_{3}^{3}$, onde $Z_{3}=2 Y_{1} Z_{1}$ e obtemos as seguintes fórmulas para computar $2 P=\left(X_{3}: Y_{3}: Z_{3}\right)$ em coordenadas Jacobianas ${ }^{1}$ :

$$
\begin{aligned}
X_{3} & =\left(3 X_{1}^{2}+a Z_{1}^{4}\right)^{2}-8 X_{1}^{2} Y_{1}^{2} \\
Y_{3} & =\left(3 X_{1}^{2}+a Z_{1}^{4}\right)\left(4 X_{1} Y_{1}^{2}-X_{3}\right)-8 Y_{1}^{4} \\
Z_{3} & =2 Y_{1} Z_{1} .
\end{aligned}
$$

Portanto, pelo armazenamento de alguns, valores intermediários pode-se calcular $X_{3}, Y_{3}$ e $Z_{3}$ com 6 elevações ao quadrado e 4 multiplicações.

A contagem das operações para adição de duplicação em vários sistemas de coordenadas são listados na Tabela 2.1. A notação $C 1+C 2 \rightarrow C 3$ significa que o primeiro ponto está na coordenada $C 1$ e o segundo na coordenada $C 2$, enquanto a soma é expressa na coordenada $C 3$.

\subsubsection{Curva elíptica $y^{2}+x y=x^{3}+a x^{2}+b$}

Muitos tipos de coordenadas projetivas tem sido propostos. Citaremos três:

\footnotetext{
${ }^{1}$ Note que $\left(X_{3}: Y_{3}: Z_{3}\right) \sim\left(X_{3}^{\prime}: Y_{3}^{\prime}: Z_{3}^{\prime}\right)$ pois $X_{3}=X_{3}^{\prime} \lambda^{2}, Y_{3}=Y_{3}^{\prime} \lambda^{3}$ e $Z_{3}=\lambda$, onde $\lambda=2 Y_{1} Z_{1}$
} 


\begin{tabular}{|c|c|c|}
\hline Duplicação & Adição geral & $\begin{array}{c}\text { Adição em } \\
\text { coordenadas mistas }\end{array}$ \\
\hline $2 \mathrm{~A} \rightarrow \mathrm{A}(1 \mathrm{I}, 2 \mathrm{M}, 2 \mathrm{~S})$ & $\mathrm{A}+\mathrm{A} \rightarrow \mathrm{A}(1 \mathrm{I}, 2 \mathrm{M}, 1 \mathrm{~S})$ & $\mathrm{J}+\mathrm{A} \rightarrow \mathrm{J}(8 \mathrm{M}, 3 \mathrm{~S})$ \\
$2 \mathrm{P} \rightarrow \mathrm{P}(7 \mathrm{M}, 4 \mathrm{~S})$ & $\mathrm{P}+\mathrm{P} \rightarrow \mathrm{P}(12 \mathrm{M}, 2 \mathrm{~S})$ & $\mathrm{J}+\mathrm{C} \rightarrow \mathrm{J}(11 \mathrm{M}, 3 \mathrm{~S})$ \\
$2 \mathrm{~J} \rightarrow \mathrm{J}(4 \mathrm{M}, 4 \mathrm{~S})$ & $\mathrm{J}+\mathrm{J} \rightarrow \mathrm{J}(12 \mathrm{M}, 4 \mathrm{~S})$ & $\mathrm{C}+\mathrm{A} \rightarrow \mathrm{C}(8 \mathrm{M}, 3 \mathrm{~S})$ \\
$2 \mathrm{C} \rightarrow \mathrm{C}(5 \mathrm{M}, 4 \mathrm{~S})$ & $\mathrm{C}+\mathrm{C} \rightarrow \mathrm{C}(11 \mathrm{M}, 3 \mathrm{~S})$ & \\
\hline
\end{tabular}

Tabela 2.1: Contagem das operações de adição e duplicação de pontos em $y^{2}=x^{3}+a x+b$, onde: $\mathrm{A}=$ afim, $\mathrm{P}=$ projetiva padrão, $\mathrm{J}=$ jacobiana, $\mathrm{C}=$ Chudnovsky, $\mathrm{I}=$ inversão, $\mathrm{M}=$ multiplicação e $\mathrm{S}=$ elevação ao quadrado (ver $[$ : $]$ ).

Coordenada projetiva padrão: $\mathrm{O}$ ponto projetivo $(X: Y: Z)$ corresponde ao ponto afim $(X / Z, Y / Z)$. A equação projetiva da curva é $Y^{2} Z+$ $X Y Z=X^{3}+a X^{2} Z+b Z^{3}$. O ponto infinito corresponde ao ponto $(0: 1: 0)$, enquanto a negação de $(X: Y: Z)$ é $(X: X+Y: Z)$.

Nota:

$$
\begin{aligned}
E: y^{2}+x y & =x^{3}+a x^{2}+b \\
& \Rightarrow Y^{2} / Z^{2}+X Y / Z^{2}=X^{3} / Z^{3}+a X^{2} / Z^{2}+b \\
& \Rightarrow Y^{2} Z+X Y Z=X^{3}+a X^{2} Z+b Z^{3} .
\end{aligned}
$$

Coordenada projetiva Jacobiana: O ponto projetivo $(X: Y: Z)$ corresponde ao ponto afim $\left(X / Z^{2}, Y / Z^{3}\right)$. A equação projetiva da curva é $Y^{2}+X Y Z=X^{3}+a X^{2} Z^{2}+b Z^{6}$. O ponto infinito corresponde ao ponto $(1: 1: 0)$, enquanto a negação de $(X: Y: Z)$ é $(X: X+Y: Z)$.

Nota:

$$
\begin{aligned}
y^{2}+x y & =x^{3}+a x^{2}+b \\
& \Rightarrow Y^{2} / Z^{6}+X Y / Z^{5}=X^{3} / Z^{6}+a X^{2} / Z^{4}+b \\
& \Rightarrow Y^{2}+X Y Z=X^{3}+a X^{2} Z^{2}+b Z^{6} .
\end{aligned}
$$

Coordenada projetiva López-Dahab: O ponto projetivo $(X: Y: Z)$ corresponde ao ponto afim $\left(X / Z, Y / Z^{2}\right)$. A equação projetiva da curva é $Y^{2}=X Y Z=X^{3} Z+a X^{2} Z^{2}+b Z^{4}$. O ponto infinito corresponde ao ponto $(1: 0: 0)$, enquanto a negação de $(X: Y: Z)$ é $(X: X+Y: Z)$.

Nota:

$$
\begin{aligned}
y^{2}+x y & =x^{3}+a x^{2}+b \\
& \Rightarrow Y^{2} / Z^{4}+X Y / Z^{3}=X^{3} / Z^{3}+a X^{2} / Z^{2}+b \\
& \Rightarrow Y^{2}+X Y Z=X^{3} Z+a X^{2} Z^{2}+b Z^{4} .
\end{aligned}
$$


A contagem das operações para adição e duplicação em vários sistemas de coordenadas são listados na Tabela 2.2.

\begin{tabular}{|c|c|c|c|}
\hline $\begin{array}{c}\text { Sistema de } \\
\text { Coordenadas }\end{array}$ & $\begin{array}{c}\text { Adição } \\
\text { geral }\end{array}$ & $\begin{array}{c}\text { Adição geral } \\
\text { (coordenadas } \\
\text { mistas) }\end{array}$ & Duplicação \\
\hline $\begin{array}{c}\text { Afim } \\
\text { Projetiva padrão }\end{array}$ & $\begin{array}{l}\mathrm{V}+\mathrm{M} \\
13 \mathrm{M}\end{array}$ & - & $\mathrm{V}+\mathrm{M}$ \\
Projetiva jacobiana & $14 \mathrm{M}$ & $10 \mathrm{M}$ & $7 \mathrm{M}$ \\
Projetiva López-Dahab & $14 \mathrm{M}$ & $8 \mathrm{M}$ & $5 \mathrm{M}$ \\
\hline
\end{tabular}

Tabela 2.2: Contagem das operações de adição e duplicação de pontos em $y^{2}+x y=x^{3}+a x^{2}+b$, onde: $\mathrm{M}=$ multiplicação e $\mathrm{V}=$ divisão (ver [s]).

\subsection{Padrões}

Dois objetivos primários dos padrões são promover a interoperabilidade e facilitar a difusão do uso de boas técnicas.

Padrões para curvas elípticas têm sido preparados por várias entidades ao redor do mundo. Alguns desses trabalhos estão sumarizados abaixo.

- ANSI X9.62 (1999): algoritmo de assinatura digital em curvas elíptịcas;

- FIPS 186.2 (2000): padrão de assinatura digital (DSS);

- IEEE 1363-2000 (2000): especificação padrão de criptografia de chave pública;

- SEC1 (2000): criptografia em curvas elípticas;

- SEC2 (2000): parâmetros recomendados para curvas elípticas;

- ISO/IEC 15946: conjunto de padrões de criptografia em curvas elípticas que inclui esquemas de assinatura, de estabelecimento de chaves e de assinatura digital com recuperação da mensagem.

Na seqüência apresentamos endereços de internet de algumas entidades de padronização e grupos de trabalhos:

- ANSI (American National Standards Institute)

http: www ansiorg

- X9 (Standards for the Financial Services Industry)

http: www xo.org 
- FIPS (Federal Information Processing Standards)

http:/ /www.itl.nist.gov/fipspubs

- P1363 (Specification for Public-Key Cryptography)

http:/grouper.iece.org/groups/1636

- SECG (Standards for Efficient Cryptography Group)

http://www.secg.org

- SEC (Standards for Efficient Cryptography Documents)

http:/www.secg.org/secg docs.htru

- WAP WTLS (Wireless Application Protocol Wireless Transport Layer Security Specification)

http://www.wapforum.org

- ISO (International Organization for Standardization)

http:/www.iso.ch

- IEC (International Electrotechnical Commission)

hitp: www.iec.ch

\subsection{Aspectos relativos a Segurança}

Os parâmetros de uma curva elíptica devem ser escolhidos de tal forma a resistir a todos os possíveis ataques no problema do logaritmo discreto em curvas elípticas (PLDCE). Esta seção resume as principais medidas a serem adotadas para contrapor a esses ataques. ${ }^{2}$

Não existe uma prova matemática de que o PLDCE seja intratável, isto é, não existe prova de que não existe um algoritmo eficiente para resolver o PLDCE. Contudo, existem evidências da sua intratabilidade em um computador clássico.

O algoritmo trivial para resolver o PLDCE é a pesquisa exaustiva, ou seja, computar a seqüência de pontos $P, 2 P, 3 P, 4 P \ldots$ até $Q$ ser encontrado. O tempo de execuçạ̃o no pior caso é $\eta$ passos e $\eta$ passos na média. Portanto, a pesquisa exaustiva pode ser evitada com um valor de $\eta$ suficientemente grande.

O melhor ataque de propósito geral conhecido no PLDCE é a combinação dos algoritmos de Pohlig-Hellman e Pollard's rho, com tempo de execução exponencial $O(p)$ onde $p$ é máximo divisor primo de $\mathcal{N}_{n}$. Para resistir a esse ataque deve-se escolher um valor de $\mathcal{N}_{n}$ que seja divisível por um número primo $p$ suficientemente grande, por exemplo $p>2^{160}$.

\footnotetext{
${ }^{2}$ Foge do escopo deste trabalho proteção contra certos tipos de ataques tais como o descrito em [2]
} 
Os ataques isomórficos tentam reduzir o PLDCE para o problema do logaritmo discreto (PLD) em um grupo isomórfico onde algoritmos de tempo subexponencial (ou mais rápidos) são conhecidos. Nesta classe estão os ataques de emparelhamento Weil e Tate (Weil and Tate pairing attacks), ataques em curvas elípticas anômalas definidas sobre um corpo primo ${ }^{3} \mathrm{e}$ o Weil descent. A matemática atrás desses ataques é bastante sofisticada, no entanto, existem medidas simples que tornam uma determinada curva elíptica imune a esses ataques.

$\mathrm{O}$ ataque em curvas elípticas anômalas definidas sobre um corpo primo pode ser facilmente contornado ao se garantir que $\not \equiv E\left(\mathbb{F}_{p}\right) \notin\{p, p+2\}$.

Para que uma curva elíptica $E$ definida sobre $\mathbb{F}_{q}$ seja imune ao ataque de emparelhamento Weil e Tate, é suficiente garantir que $\eta$, a ordem do ponto base $P \in E\left(\mathbb{F}_{q}\right)$ não divida $q^{r}-1$ para todos pequenos $r$ em que o PLD em $\mathbb{F}_{q^{r}}$ seja tratável. Se $\eta>2^{160}$, então é suficiente verificar essa condição para todos $r \in[1,20]$.

Para garantir proteção contra o Weil descent deve-se selecionar curvas definidas sobre um corpo $\mathbb{F}_{2^{r}}$ aleatório, onde $r \in[160,600]$. Entretanto, para se precaver contra futuros desenvolvimentos desta metodogia, é prudente evitar usar um $r$ que seja composto.

\subsection{Conclusão}

Neste capítulo foram apresentados os principais conceitos relacionados as curvas elípticas, tais como a história do seu surgimento, a dificuldade matemática que torna o seu uso adequado para aplicações criptográficas, sua definição, suas equações e fórmulas, os sistemas de coordenadas utilizados para representar pontos da curvas, os padrões atuais que fazem uso da mesma e as questões relacionadas ao seu emprego seguro.

Nossa contribuição foi organizar um vasto material disponível na literatura sobre o assunto, de forma compacta e objetiva, mas com a profundidade suficiente para a compreensão dos capítulos subseqüentes.

\footnotetext{
${ }^{3}$ Em uma curva anômala o módulo do endomorfismo de Frobenius é igual a 1.
} 


\section{Capítulo 3}

\section{Métodos gerais de multiplicação em curvas elípticas}

Em um sistema de criptografia de chave pública ocorre com grande freqüência a elevação de elementos de um grupo (por exemplo: $\mathbb{F}_{2^{m}}, \mathbb{Z}_{n}$ ) a grandes potências. Um ponto básico é a rapidez com que esta exponenciação pode ser feita, o que freqüentemente determina se um dado sistema é prático.

O melhor método de exponenciação depende do grupo que está sendo usado, do hardware sobre o qual está sendo implementado, e se um elemento está sendo repetidamente elevado a potências diferentes $\left(g^{r_{x}}\right)$, diferentes elementos estão sendo elevados a uma potência fixa $\left(g_{y}^{r}\right)$, ou ambos potências e elementos do grupo variam $\left(g_{y}^{r_{x}}\right)$.

Quando falamos em métodos de exponenciação estamos tratando de grupos multiplicativos: O grupo das curvas elípticas é aditivo, porém os dois pontos de vista são equivalentes.

Pretendemos fazer um levantamento de alguns métodos de exponenciação conhecidos, examinando suas forças e fraquezas, bem como mostrar sua versão adaptada ao grupo aditivo curvas elípticas. Veremos os métodos binário, $s$-ário, janela deslizante, BGMW .

\subsection{Cadeias aditivas}

Uma cadeia aditiva é útil no calculo $g^{r}$, onde $r$ é um expoente fixo e $g$ varia $\left(g_{y}^{r}\right)$. Como exemplo da aplicação de cadeias aditivas temos o RSA em que diferentes números podem ser elevados a uma potência fixa $\left(g_{y}^{r}\right)$.

A questão básica em cadeias aditivas é encontrar qual o menor número de multiplicações necessárias para computar $g^{r}$, dado que a única operação permitida é multiplicar duas potências já computadas. Esta questão é equivalente à seguinte: qual é o comprimento da menor cadeia aditiva de $r$ ? 
Uma cadeia aditiva para $r$ é a lista de inteiros positivos

$$
a_{1}=1, a_{2}, \ldots, a_{l}=r
$$

tal que para cada $i>1$, existe algum $j$ e $k(1 \leq j \leq k<i)$ tal que $a_{i}=a_{j}+a_{k}$. Uma cadeia aditiva curta para $r$ fornece um algoritmo rápido para computar $g^{r}$ : compute $g^{a_{2}}, g^{a_{3}}, \ldots, g^{a_{l-1}}, g^{r}$.

Exemplo 3.1. Uma cadeia aditiva para $r=10$ é 1,2, 4, 5, 10 pois podemos obter $g^{10}$ computando $g^{1}, g^{2}, g^{4}, g^{5}$ e $g^{10}$.

Seja $L(r)$ o comprimento da menor cadeia aditiva de $r$. O valor exato de $L(r)$ é conhecido apenas para pequenos valores de $r$. Para grandes $r$,

$$
\left(L(r)=\log _{2} r+(1+o(1)) \frac{\log _{2} r}{\log _{2} \log _{2} r}\right)
$$

O limite inferior foi mostrado por Erdös [5] usando um argumento de contagem, e o limite superior é dado pelo método $s$-ário que será descrito na seção 3.4 .

Encontrar a melhor cadeia aditiva é impraticável, mas podemos encontrar valores próximos do valor ótimo. Apresentaremos algoritmos eficientes que produzirão cadeias aditivas razoáveis.

\subsection{Seqüências aditivas}

Seqüências aditivas são usadas quando $g$ é elevado a múltiplas potências $\left(g^{r_{x}}\right)$. Elas podem ser usadas também para acelerar métodos, tais como o método janela deslizante. Nestes métodos, um certo número de potências $g^{r_{1}}, \ldots, g^{r_{t}}$ são primeiramente computados.

Em outro exemplo da aplicação de seqüências aditivas temos a troca de chaves Diffie-Hellman, onde um número fixo é elevado a diferentes potências $\left(g^{r_{x}}\right)$.

Uma sequência aditiva para $r_{1}, r_{2}, \ldots, r_{t}$ é uma cadeia aditiva

$$
a_{1}=1, a_{2}, \ldots, a_{l}
$$

que contém $r_{1}, r_{2}, \ldots, r_{t}$.

Yao [d] mostrou que o comprimento mínimo de uma seqüência aditiva para $r_{1}, r_{2}, \ldots, r_{t}$ é

$$
\left(L\left(r_{1}, \ldots, r_{t}\right)=\log _{2} r+(t+o(1)) \frac{\log _{2} r}{\log _{2} \log _{2} r}\right)
$$

onde $r=\max \left(r_{1}, r_{2}, \ldots, r_{t}\right)$. Bos e Coster [] apresentaram heurísticas para a construção de boas seqüências aditivas. 


\subsection{Método binário}

Este método é também conhecido como o método da elevação ao quadrado e da multiplicação e é baseado no conceito de cadeias aditivas. Tem mais ou menos 2000 anos; Knuth [0] discute a sua história e fornece referências. A idéia básica é computar $g^{r}$ usando a expansão binária de $r$.

Dada a expansão binária $r=\sum_{i=0}^{t} k_{i} 2^{i}, g^{r}$ pode ser computado através do algoritmo 3.1.

Algoritmo 3.1 (Método Binário: versão esquerda para direita):

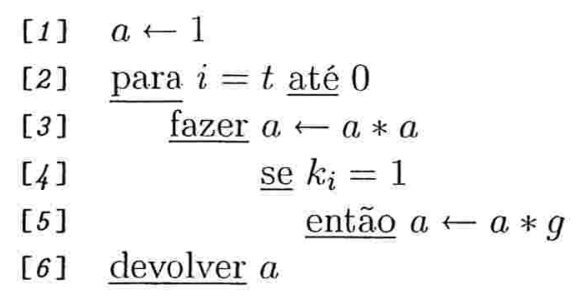

Exemplo 3.2. $r=10=\langle 1010\rangle_{2}$

\begin{tabular}{|l|l|l|l|}
\hline 1 & \multicolumn{1}{c}{0} & 1 & 0 \\
\hline$g$ & $g^{2}$ & $g^{5}$ & $g^{10}$ \\
\hline
\end{tabular}

A cada passo do loop a é igual a $g^{s}$, onde a representação binária de $s$ é o prefixo da representação binária de $r$. A elevação de $a$ ao quadrado tem o efeito de dobrar $s$, e a multiplicação por $g$ colocar 1 no último dígito.

A versão direita para esquerda do algoritmo tem a vantagem de não exigir o conhecimento de $t$ antes do tempo.

Este algoritmo consome no pior caso $2\left\lfloor\log _{2} r\right\rfloor$ multiplicações e $3\left\lfloor\log _{2} r\right\rfloor / 2$ multiplicações na média.

Algoritmo 3.2 (Método Binário: versão direita para esquerda):

[1] $a \leftarrow 1$

[2] para $i=0$ até $t$

[3] fazer se $k_{i}=1$

[4] $\quad$ então $a \leftarrow a * g$

[5] $\quad a \leftarrow a * a$

[6] devolver $a$.

Exemplo 3.3. $r=10=\langle 1010\rangle_{2}$

\begin{tabular}{|c|c|c|c|}
\hline 1 & \multicolumn{1}{c}{0} & 1 & 0 \\
\hline$g^{10}$ & $g^{4}$ & $g^{2}$ & 1 \\
\hline
\end{tabular}




\subsection{Método s-ário}

O método anterior possui uma generalização óbvia: usar uma base maior do que 2, o que diminui o comprimento da expansão de $r$ e implica em uma menor quantidade de multiplicações à custa de alguma pré-computação. O método $s$-ário é baseado no conceito de seqüências aditivas.

Seja a expansão $s$-ária $r=\sum_{i=0}^{t^{\prime}} k_{i} s^{i}$. O algoritmo 3.3 computa $g^{r}$ :

Algoritmo 3.3 (Método s-ário):

[1] Pré-compute $g^{2}, g^{3}, \ldots, g^{s-1}$

[2] $a \leftarrow 1$

[3] para $i=t^{\prime}$ até 0

[4] fazer $a \leftarrow a^{s}$

[5] $\quad a \leftarrow a * g^{k_{i}}$

[6] devolver $a$

Este método é particularmente atrativo quando $s=2^{w}$ tal que elevar $a$ à $s$-ésima potência envolve somente $w$ elevações ao quadrado. Neste caso, o número de multiplicações é de no máximo

$$
2^{w}-2+\left\lfloor\log _{2} r\right\rfloor+\left\lfloor\log _{2} r\right\rfloor / w
$$

sendo $\left(2^{w}-2\right)$ multiplicações na pré-computação, $\left\lfloor\log _{2} r\right\rfloor$ elevações ao quadrado, e no máximo $\left\lfloor\log _{2} r\right\rfloor / w$ multiplicações (na média um pouco menor, desde que alguns dos $k_{i}$ serão zero).

Fazendo $w=\log _{2} \log _{2} r-2 \log _{2} \log _{2} \log _{2} r$, obtemos o limite superior da equação (3.1).

Exemplo 3.4. $r=100=\langle 1210\rangle_{4}$

\begin{tabular}{|c|c|c|c|}
\hline 1 & 2 & 1 & 0 \\
\hline$g^{1}$ & $g^{6}$ & $g^{25}$ & $g^{100}$ \\
\hline
\end{tabular}

O método $2^{w}$-ário pode ser interpretado como se tomássemos janelas de $w$ bits na representação binária de $r$, calculássemos as potências na janela uma por uma, fazendo-se elevações ao quadrado $w$ vezes através do deslocamento cíclico sobre elas mesmas e então multiplicássemos pela potência na próxima janela.

Exemplo 3.5. $r=100, s=2^{w}$, onde $w=2$

$100=$\begin{tabular}{|c|c|c|c|}
1 & \multicolumn{1}{c}{10} & 01 & 00 \\
\hline$g^{1}$ & $g^{2}$ & $g^{1}$ & $g^{0}$ \\
\hline$g^{4}$ & $g^{6}$ & $g^{25}$ & $g^{100}$ \\
\hline & $g^{24}$ & $g^{100}$ & \\
\hline
\end{tabular}




\subsection{Método janela deslizante}

A sistemática do método $2^{w}$-ário implica algumas generalizações. Uma óbvia é que não há razão para forçar as janelas estarem uma seguida da outra de tal forma que seqüências de zeros não necessitam ser calculadas. Além disso, somente potências ímpares de $g$ necessitam ser pré-computadas.

A representação binária de $r=26235947428953663183191$ é

$1011000111001000000111010010100111010 \cdots$

$$
\cdots 10000001011110000011111001100101010111
$$

No método $s$-ário, a escolha ótima, no caso deste número de 75 bits, é $s=2^{3}=8$. O custo é de 102 multiplicações.

No método janela deslizante, com janelas de tamanho $\leq w=4$, o número de multiplicações é de somente 93: 8 multiplicações para computar as potências ímpares até $15\left(g^{1}, g^{2}, g^{3}, g^{5}, g^{7}, g^{9}, g^{11}, g^{13}, g^{15}\right)$, 71 elevações ao quadrado, e 14 multiplicações dos valores intermediários:

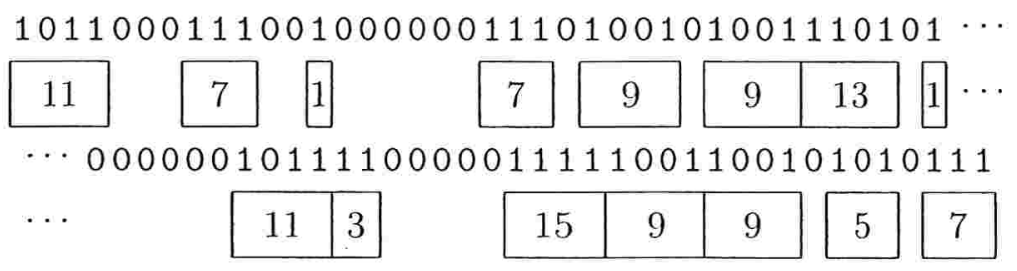

\subsection{Método BGMW}

Em casos como a troca de chaves Diffie-Hellman, onde um número fixo é elevado repetidamente a diferentes potências $\left(g^{r_{x}}\right)$, a pré-computação de algumas potências é uma opção para acelerar a exponenciação. Esta observação foi feita por Brickell, Gordon, McCurley e Wilson [?].

Os autores mostraram que pode ser economizado mais tempo multiplicando primeiro as potências com coeficientes iguais, para somente depois elevar os subprodutos de potências aos respectivos coeficientes.

Suponha que $r=\sum_{i=0}^{t^{\prime}-1} a_{i} x_{i}$, onde $0 \leq a_{i} \leq h$, então

$$
g^{r}=\prod_{d=1}^{h} c_{d}^{d}
$$

onde $c_{d}=\prod_{i: a_{i}=d} g^{x_{i}}$.

Esta equação pode ser computada com mais eficiência, da seguinte forma

$$
\prod_{d=1}^{h} c_{d}^{d}=c_{h}\left(c_{h} c_{h-1}\right)\left(c_{h} c_{h-1} c_{h-2}\right) \ldots\left(c_{h} c_{h-1} \ldots c_{1}\right)
$$


Exemplo 3.6. $s=4$ e $r=110=\langle 1232\rangle_{4}$.

$$
\begin{aligned}
& c_{1}=\prod_{i: a_{i}=1} g^{x_{i}}=g^{4^{3}} \\
& c_{2}=\prod_{i: a_{i}=2} g^{x_{i}}=g^{4^{2}} g^{4^{0}} \\
& c_{3}=\prod_{i: a_{i}=3} g^{x_{i}}=g^{4^{1}} \\
& g^{r}=\prod_{d=1}^{3} c_{d}^{d}=c_{1}^{1} c_{2}^{2} c_{3}^{3}=\left(g^{4^{3}}\right)^{1}\left(g^{4^{2}} g^{4^{0}}\right)^{2}\left(g^{4^{1}}\right)^{3}
\end{aligned}
$$

Pela equação 3.4 obtemos, de maneira mais eficiente, o mesmo resultado

$$
\prod_{d=1}^{3} c_{d}^{d}=c_{3}\left(c_{3} c_{2}\right)\left(c_{3} c_{2} c_{1}\right)=g^{4^{1}}\left(g^{4^{1}}\left(g^{4^{2}} g^{4^{0}}\right)\right)\left(g^{4^{1}}\left(g^{4^{2}} g^{4^{0}}\right) g^{4^{3}}\right)
$$

O próximo teorema é o Lema 01 de [2]:

Teorema 3.1. Suponha $r=\sum_{i=0}^{t^{\prime}-1} a_{i} x_{i}$, onde $0 \leq a_{i} \leq h$, e $g^{x_{i}}$ tenha sido pré-computado para cada $0 \leq i<t^{\prime}$. O algoritmo 3.4 computa $g^{r}$ com $t^{\prime}+h-2$ multiplicações.

Algoritmo 3.4 (Método BGMW):

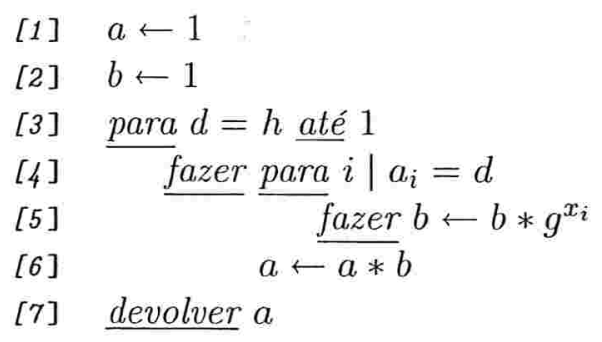

Fazendo $x_{i}=b^{i} \operatorname{com} b=\left\lfloor\left(\log _{2} r\right) / \log _{2}^{2} r \log _{2} r\right\rfloor$, este algoritmo computará $g^{r}$ em $(1+\mathcal{O}(1)) \log _{2} r / \log _{2} \log _{2} r$ multiplicações com $\mathcal{O}\left(\log _{2} r / \log _{2} \log _{2} r\right)$ pré-computações de potências.

Exemplo 3.7. $s=4$ e $r=110=\langle 1232\rangle_{4}$

$$
\begin{aligned}
& d=3 \quad b=g^{4^{1}} \quad a=g^{4^{1}} \\
& d=2 \quad b=g^{4^{1}}\left(g^{4^{1}} g^{4^{0}}\right) \quad a=g^{4^{1}}\left(g^{4^{1}}\left(g^{4^{2}} g^{4^{0}}\right)\right) \\
& d=1 \quad b=\left(g^{4^{1}}\left(g^{4^{0}}\right)\right)\left(g^{4^{3}}\right) \\
& a=\left(g^{4^{1}}\left(g^{4^{1}}\left(g^{4^{2}} g^{4^{0}}\right)\right)\right)\left(g^{4^{1}}\left(g^{4^{2}} g^{4^{0}}\right)\right)\left(g^{4^{3}}\right)
\end{aligned}
$$




\subsection{Adaptação dos métodos para curvas elípticas}

Em curvas elípticas, um grupo aditivo, computa-se $K P$, onde $K \in \mathbb{Z}$ e $P$ é um ponto da curva definida sobre $\mathbb{F}_{q}$. Esta operação é denominada de multiplicação escalar de um ponto da curva.

As operações de multiplicação e elevação ao quadrado em um grupo multiplicativo correspondem a soma e duplicação em um grupo aditivo.

Assumimos que os pontos $P$ e $Q$ tenham ordem $\eta \leq \mathcal{N}_{n}$, e portanto os multiplicadores $K$ são aleatoriamente selecionados de inteiros no intervalo $[1, \eta-1]$.

\subsubsection{Cadeias de adição e subtração}

Morais e Olivos [1]] observaram que as cadeias de adição-subtração podem ser úteis em curvas elípticas, uma vez que a negação de um ponto possui custo desprezível. Nas curvas $y^{2}=x^{3}+a x+b$ definidas sobre $\mathbb{F}_{p}$ com $p>3$, o negativo de $(x, y)$ é $(x,-y)$. Nas curvas $y^{2}+x y=x^{3}+a x^{2}+b$ definidas sobre $\mathbb{F}_{2^{r}}$, o negativo é $(x, x+y)$.

Uma maneira de reduzir o comprimento de uma cadeia aditiva é permitir outras operações, tais como a subtração. Por exemplo, a menor cadeia aditiva para 31 é

$$
1,2,3,5,10,11,21,31
$$

mas se a subtração é permitida obtemos uma cadeia menor:

$$
1,2,4,8,16,32,31 \text {. }
$$

A Maioria dos algoritmos de cadeia aditiva podem ser generalizados para. cadeias de adição-subtração.

\subsubsection{Forma não-adjacente (NAF)}

Como mencionado anteriormente, o negação de pontos em curvas elípticas possui custo desprezível, tal que cadeias de adição e subtração podem ser usadas, o que sugere o uso de uma representação que permita dígitos negativos. Seja a representação

$$
K=\sum_{i=0}^{l} k_{i} 2^{i}
$$

onde $k_{i} \in\{0, \pm 1\}$ para todos os $i$. Consideremos como peso de uma representação o número de $k_{i}$ 's diferentes de zero e $w(k)$ o peso mínimo de qualquer representação de $K$. A forma NAF é uma representação binária de $K \operatorname{com} k_{i} k_{i+1}=0$ para todos os $i \geq 0$.

A vantagem de se usar o NAF é que em geral possui menor número de coeficientes não-zero do que a representação binária comum, reduzindo o 
número de multiplicações. Morain e Olivos [1] mostraram que o número esperado de não-zeros em um NAF de comprimento $l$ é $l / 3$.

Exemplo 3.8. Seja $K=1122334455$ e $\overline{1}=-1$. Então,

$$
\begin{aligned}
\langle K\rangle_{2} & =1000010111001010111011011110111 \\
\mathrm{NAF}_{2}(K) & =100010 \overline{1} 00 \overline{1} 010 \overline{1} 0 \overline{1} 000 \overline{1} 00 \overline{1} 0000 \overline{1} 00 \overline{1}
\end{aligned}
$$

\section{Propriedades do $\operatorname{NAF}(K)$ :}

1. Um inteiro K possui uma única representação denotada $\operatorname{NAF}(K)$;

2. $\operatorname{NAF}(K)$ tem a menor quantidade de dígitos diferentes de zero de todas as representações digitais com sinal de $K$;

3. O comprimento do $\operatorname{NAF}(K)$ é no máximo o comprimento da representação binária de $K$ mais 1 [17];

4. Se o comprimento do $\operatorname{NAF}(K)$ é $l$, então $2^{l} / 3<K<2^{l+1} / 3$;

5. A densidade média de dígitos diferentes de zero entre todos os $\operatorname{NAF}(K)$ de comprimento $l$ é de aproximadamente $l / 3$.

$\mathrm{O} \operatorname{NAF}(K)$ pode ser eficientemente computado pelo algoritmo 3.5 . Os dígitos do $\operatorname{NAF}(K)$ são gerados pela repetição contínua da divisão de $K$ por 2 , permitindo restos em $\{0, \pm 1\}$. Se $K$ é ímpar, então o resto $r \in\{0, \pm 1\}$ é escolhido de tal forma que $K$ na próxima iteração seja par. Isto garante que o próximo dígito do $\mathrm{NAF}(K)$ seja 0 .

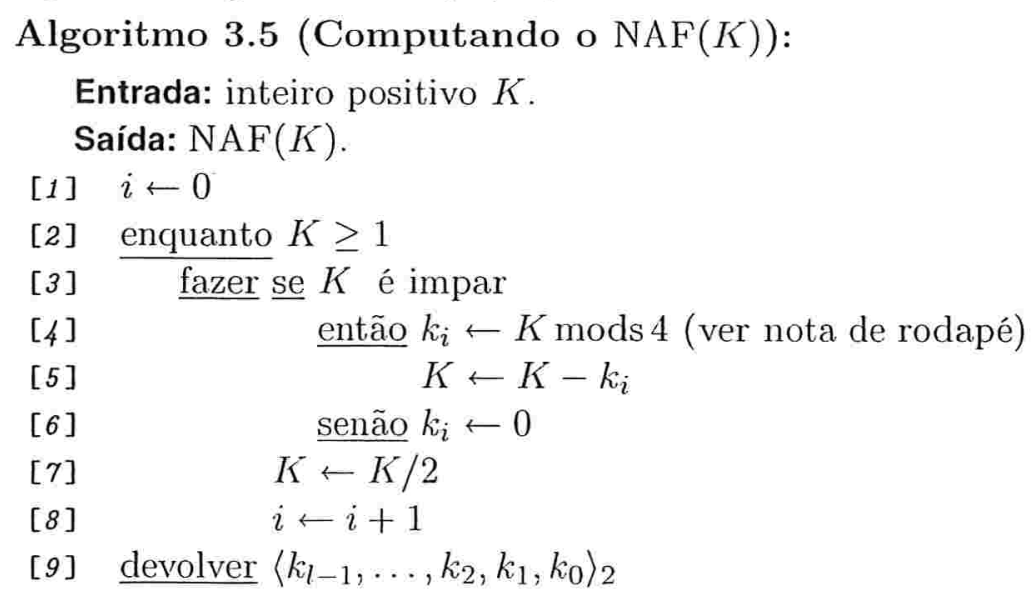

Neste algoritmo, a linha (4) decorre do fato que $K$ ser divisível por 4 se somente se $K \equiv 0 \bmod 4$. Assim sendo, o $k_{i}$ obtido nesta linha torna o valor de $K$ da linha (5) divisível por 4 . Portanto na próxima iteração $K$ será par.

\footnotetext{
${ }^{0}$ A notação "mods" indica que a redução modular retorna o menor resíduo em valor absoluto, ou seja, se $a=b \operatorname{mods} c$, então $-c / 2 \leq a<c / 2$.
} 
Exemplo 3.9. $r=7=\langle 111\rangle_{2}$.

\begin{tabular}{|l|l|l|l|}
1 & 2 & 4 & 7 \\
\hline 1 & 0 & 0 & $\overline{1}$ \\
\hline
\end{tabular}

\subsubsection{Método binário}

Os algoritmos 3.6 e 3.7 são versões aditivas do método da elevação ao quadrado e da multiplicação para exponenciação — algoritmo 3.1 (página 19) e algoritmo 3.2 (página 19).

O algoritmo 3.6 processa os bits de $K$ da esquerda para a direita, enquanto que o algoritmo 3.7 seguinte processa os bits da direita para a esquerda.

Algoritmo 3.6 (Método binário: versão esquerda para direita):

Entrada: $K=\left\langle k_{t-1}, \ldots, k_{2}, k_{1}, k_{0}\right\rangle_{2}, P \in E\left(\mathbb{F}_{q}\right)$.

Saída: $K P$.

[1] $Q \leftarrow \mathcal{O}$

[2] para $i=t-1$ até 0

[3] fazer $Q \leftarrow 2 Q$

[4] $\quad$ se $k_{i}=1$

[5] $\quad$ então $Q \leftarrow Q+P$

[6]

[7] devolver $Q$

Algoritmo 3.7 (Método binário: versão direita para esquerda):

Entrada: $K=\left\langle k_{t-1}, \ldots, k_{2}, k_{1}, k_{0}\right\rangle_{2}, P \in E\left(\mathbb{F}_{q}\right)$.

Saída: $K P$.

[1] $Q \leftarrow \mathcal{O}$

[2] para $i=0$ até $t-1$

[3] $\quad$ fazer se $k_{i}=1$

[4] $\quad$ então $Q \leftarrow Q+P$

[5] $\quad P \leftarrow 2 P$

[6] devolver $Q$

A densidade média da representação binária de $K$ é $1 / 2$, portanto o tempo de execução esperado dos algoritmos 3.4 e 3.5 é de aproximadamente $m / 2$ adições de pontos $(A)$ e $m$ duplicações de pontos $(D)$ denotado por

$$
(m / 2) A+(m) D .
$$

onde $m=\log _{2} \eta$ 


\subsubsection{Método NAF binário}

O algoritmo 3.8 usa o $\mathrm{NAF}(K)$ ao invés da representação binária de $K$. Segue das propriedades (3) e (5) do $\operatorname{NAF}(K)$ que o tempo de execução esperado deste algoritmo é aproximadamente

$$
(m / 3) A+(m) D
$$

onde $m=\log _{2} \eta$

\section{Algoritmo 3.8 (Método NAF binário):}

Entrada: inteiro positivo $K, P \in E\left(\mathbb{F}_{q}\right)$.

Saída: $K P$.

Use o algoritmo 3.5 para computar $\operatorname{NAF}(K)=\sum_{i=0}^{l-1} k_{i} 2^{i}$.

[1] $Q \leftarrow \mathcal{O}$

[2] para $i=l-1$ até 0

[3] $\underline{\text { fazer }} Q \leftarrow \overline{2 Q}$

[4] $\quad$ se $k_{i}=1$

[5] $\quad$ então $Q \leftarrow Q+P$

[6] se $k_{i}=-1$

[7] então $Q \leftarrow Q-P$

[8]

[9] devolver $Q$

Exemplo 3.10. $r=7=100 \overline{1}$.

\begin{tabular}{|c|c|c|c|}
\hline 1 & 0 & 0 & $\overline{1}$ \\
\hline$P$ & $2 P$ & $4 P$ & $7 P$ \\
\hline
\end{tabular}

\subsubsection{Forma não-adjacente de dimensão $w$}

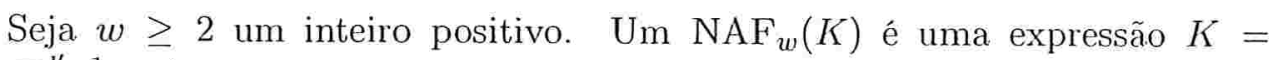
$\sum_{i=0}^{l^{\prime}-1} k_{i} 2^{i}$ onde cada coeficiente $k_{i}$ é ímpar $\left|k_{i}\right|<2^{w-1}, k_{l^{\prime}-1} \neq 0$, e no máximo um de quaisquer $w$ dígitos consecutivos é diferente de zero.

Exemplo 3.11. Seja $K=1122334455$.

$$
\begin{aligned}
K & =1000010111001010111011011110111 \\
\mathrm{NAF}_{2}(K) & =100010 \overline{1} 00 \overline{1} 010 \overline{1} 0 \overline{1} 000 \overline{1} 00 \overline{1} 0000 \overline{1} 00 \overline{1} \\
\mathrm{NAF}_{3}(K) & =100000300 \overline{1} 001003000 \overline{1} 00 \overline{1} 0000 \overline{1} 001
\end{aligned}
$$

Propriedades do $\mathrm{NAF}_{w}(K)$ :

1. Todo $K$ possui um único $\operatorname{NAF}_{w}(K)$;

2. $\operatorname{NAF}_{2}(K)=\operatorname{NAF}(K)$; 
3. O comprimento do $\mathrm{NAF}_{w}(K)$ é de no máximo o comprimento da representação binária de $K$ mais 1 ;

4. A densidade média de dígitos diferentes de zero entre todos os $\operatorname{NAF}_{w}(K)$ de comprimento $l^{\prime}$ é de aproximadamente $l^{\prime} /(w+1)$.

$\mathrm{O} \mathrm{NAF}_{w}(K)$ pode ser eficientemente computado usando o algoritmo 3.9 onde $K \operatorname{mods} 2^{w}$ são os inteiros $u$ satisfazendo $u \equiv K\left(\bmod 2^{w}\right) \mathrm{e}-2^{w-1} \leq$ $u \leq 2^{w-1}$. Os dígitos do $\operatorname{NAF}_{w}(K)$ são obtidos pela repetição continua da divisão de $K$ por 2 , permitindo restos $r$ em $\left[-2^{w-1}, 2^{w-1}-1\right]$. Se $K$ é ímpar, então o resto $r=K$ mods $2^{w}$ é escolhido, de forma que $(K-r) / 2$ seja. divisível por $2^{w-1}$, garantindo que os próximos $w-1$ dígitos sejam zero.

Algoritmo 3.9 (Computa o $\mathrm{NAF}_{w}(K)$ de um inteiro positivo):

Entrada: inteiro positivo $K$.

Saída: $\mathrm{NAF}_{w}(K)$.

[1] $i \leftarrow 0$

[2] enquanto $K \geq 1$

[3] fazer $\underline{\text { se }} K$ é impar

[4] então $k_{i} \leftarrow K\left(\operatorname{mods} 2^{w}\right)$

[5] $\quad K \leftarrow K-k_{i}$

[6] Senão $k_{i} \leftarrow 0$

[7] $\quad K \leftarrow K / 2$

[8] $\quad i \leftarrow i+1$

[9] devolver $\left(k_{l^{\prime}-1}, \ldots, k_{2}, k_{1}, k_{0}\right)$

\subsubsection{Método $2^{w}$-ário NAF}

O Algoritmo 3.10 é uma versão aditiva do método $2^{w}$-ário e generaliza o método NAF binário usando $\operatorname{NAF}_{w}(K)$ ao invés do $\operatorname{NAF}(K)$.

Algoritmo 3.10 (Método $2^{w}$-ário NAF):

Entrada: inteiro positivo $K$ e $P \in E\left(\mathbb{F}_{q}\right)$.

Saída: $K P$.

Use o algoritmo 3.9 para computar $\operatorname{NAF}_{w}(K)=\sum_{i=0}^{l^{\prime}-1} k_{i} 2^{i}$.

Compute $P_{i}=i P$ para $i \in\left\{1,3,5, \ldots, 2^{w-1}-1\right\}$.

[1] $Q \leftarrow \mathcal{O}$

[2] para $i=l^{\prime}-1$ até 0

[3] fazer $Q \leftarrow 2 Q$

[4] se $k_{i} \neq 0$

[5] $\quad$ então $\underline{\text { se }} k_{i}>0$

[6] $\quad$ então $Q \leftarrow Q+P_{k_{i}}$

[7] $\quad$ senão $Q \leftarrow Q-P_{-k_{i}}$

[8] devolver $Q$ 
Segue das propriedades (3) e (4) do $\mathrm{NAF}_{w}(K)$ que o tempo esperado do Algoritmo 3.10 é de aproximadamente:

$$
\left(\left(2^{w-2}-1\right)+m /(w+1)\right) A+(m+1) D
$$

onde $m=\log _{2} \eta$

\subsubsection{Método janela deslizante}

Uma janela deslizante $\leq w$ pode ser usada no NAF de $K$, conduzindo ao Algoritmo 3.11. A janela move da esquerda para a direita sobre os dígitos no $\operatorname{NAF}(K)$, com a certificação de que o valor na janela seja ímpar de forma a para reduzir a quantidade de pré-computação.

\section{Algoritmo 3.11 (Método Janela deslizante):}

Entrada: Janela de dimensão $w$, inteiro positivo $K, P \in E\left(\mathbb{F}_{\boldsymbol{q}}\right)$.

Saída: $K P$.

Use o Algoritmo 3.5 para computar $\operatorname{NAF}(K)=\sum_{i=0}^{l-1} k_{i} 2^{i}$.

Compute $P_{i}=i P$ para $i \in\left\{1,3, \ldots, \frac{2\left(2^{w}-(-1)^{w}\right)}{3}-1\right\}$.

[1] $Q \leftarrow \mathcal{O}$

[2] $i \leftarrow l-1$

[3] enquanto $i \geq 0$

[4] fazer $\underline{\text { se }} k_{i}=0$

[5] então $x \leftarrow 1$

[6] $u \leftarrow 0$

[7] Senão encontrar o máximo $x \leq w$

[8] tal que $u \leftarrow\left(k_{i}, \ldots, k_{i-x+1}\right)$ seja ímpar

[9] $Q \leftarrow 2^{x} Q$

[10] $\quad$ se $u>0$

[11] $\quad$ então $Q \leftarrow Q+P_{u}$

[12] senão se $u<0$

[13] então $Q \leftarrow Q-P_{-u}$

[14]

[15]

[16] devolver $Q$

Exemplo 3.12. $r=100, w=3$ e $N A F(100)=10 \overline{1} 00100$.

\begin{tabular}{|c|c|c|c|c|c|c|c|}
\hline & 6 & 5 & 4 & 3 & 2 & 1 & 0 \\
\hline 1 & 0 & -1 & 0 & 0 & 1 & 0 & 0 \\
\hline \multicolumn{3}{|c|}{$3 \mathrm{P}$} & $6 \mathrm{P}$ & $12 \mathrm{P}$ & $25 \mathrm{P}$ & $50 \mathrm{P}$ & $100 \mathrm{P}$ \\
\hline
\end{tabular}

O tamanho médio de uma seqüência de zeros entre janelas no método janela deslizante é

$$
v(w)=\frac{4}{3}-\frac{(-1)^{w}}{3 \cdot 2^{w-2}}
$$


Segue-se que o tempo de execução esperado do Algoritmo 3.11 é de aproximadamente

$$
\left[\left(\frac{2^{w}-(-1)^{w}}{3}-1\right)+\left(\frac{m}{w+v(w)}\right)\right] A+(m+1) D
$$

onde $m=\log _{2} \eta$

\subsubsection{Método BGMW}

O Algoritmo 3.12 é uma adaptação do Algoritmo 3.4. Seja $\left\langle k_{d-1}, \ldots, k_{1}, k_{0}\right\rangle_{2^{w}}$ a representação na base $2^{w}$ de $K$, onde $d=\lceil t / w\rceil$ e $t \leq m=\log _{2} \eta$ é o comprimento da expansão binária de $K$. Seja também $Q_{j}=\sum_{i: k_{i}=j} 2^{\text {wi } P}$ para cada $j, 1 \leq j \leq 2^{w}-1$.

$$
\begin{aligned}
& K P=\sum_{j=1}^{2^{w}-1} j Q_{j}, \quad \text { onde } Q_{j}=\sum_{i: k_{i}=j} 2^{w i} P \\
& K P=Q_{2^{w}-1}+\left(Q_{2^{w}-1}+Q_{2^{w}-2}\right)+\cdots+\left(Q_{2^{w}-1}+Q_{2^{w}-2}+\cdots+Q_{1}\right)
\end{aligned}
$$

Algoritmo 3.12 (Método BGMW):

Entrada: Janela $w, d=\lceil t / w\rceil, K=\left\langle k_{d-1}, \ldots, k_{1}, k_{0}\right\rangle_{2^{w}}, P \in E\left(\mathbb{F}_{q}\right)$.

Saída: $K P$.

Pré-computação: Compute $P_{i}=2^{w i} P$ para $1 \leq i \leq d-1$.

[1] $A \leftarrow \mathcal{O}, \quad B \leftarrow \mathcal{O}$

[2] para $j=2^{w}-1$ até 1

[3] fazer para $i \mid \overline{k_{i}}=j$

[4] $\underline{\text { fazer }} B \leftarrow B+P_{i}$

[5] $\quad A \leftarrow A+B$

[6] devolver $A$

O tempo esperado do algoritmo é de aproximadamente $\left(2^{w}+d-3\right) A$ onde $d=\lceil m / w\rceil$.

Exemplo 3.13. $w=2, l=7, d=4$ e $K=110=\langle 1232\rangle_{4}=\langle 1101110\rangle_{2}$. Pré-computação: $P_{1}=2^{2} P, P_{2}=2^{4} P, P_{3}=2^{16} P$.

$$
\begin{array}{rlrl}
j=3 & B & =4 P \quad A=4 P \\
j=2 & B & =4 P+(16 P+P) \quad A=4 P+(4 P+(16 P+P)) \\
j=1 & B & =64 P+(4 P+(16 P+P)) \\
& & A=4 P+(4 P+(16 P+P))+64 P+(4 P+(16 P+P))
\end{array}
$$

\subsection{Conclusão}

Neste capítulo foram apresentados os principais métodos gerais de multiplicação em curvas elípticas. São métodos gerais porque não fazem uso de 
qualquer característica particular das curvas elípticas e portanto podem ser utilizados em qualquer curva. Vimos o método binário, o método NAF Binário, o método $2^{w}$-ário NAF, o método janela deslizante e o método BGMW.

Nossa contribuição foi apresentar de maneira coerente os principais algoritmos deste tipo, permitindo que se verifique a evolução da lógica computacional de um para outro. Contribuímos também com a apresentação de exemplos de funcionamento de cada um deles. 


\section{Capítulo 4}

\section{Curvas elípticas com boas propriedades criptográficas}

O objetivo deste capítulo é descrever o artigo [10] de Koblitz que propõe o uso do endomorfismo de Frobenius para acelerar a multiplicação escalar em curvas elípticas anômalas definidas em corpos de característica 2.

$\mathrm{O}$ autor de maneira particular propõe métodos para acelerar a multiplicação em curvas de definidas sobre o corpo $\mathbb{F}_{2}$.

\subsection{Endomorfismo de Frobenius}

Seja uma curva elíptica $E$ definida sobre o corpo $\mathbb{F}_{q}$. O endomorfismo ${ }^{1}$ de Frobenius $\varphi$ é o mapeamento sobrejetivo $\varphi: E \rightarrow E$, tal que $\varphi(x, y)=$ $\left(x^{q}, y^{q}\right)$ e $\varphi(\mathcal{O})=\mathcal{O}$. Portanto, se $P_{1}=\left(x_{1}, y_{1}\right)$ e $P_{2}=\left(x_{2}, y_{2}\right)$, com $P_{1}, P_{2} \in E$, temos que $\varphi\left(P_{1}+P_{2}\right)=\varphi\left(P_{1}\right)+\varphi\left(P_{2}\right)$ e $\varphi\left(K P_{1}\right)=K \varphi\left(P_{1}\right)$, onde $K \in \mathbb{Z}$.

O endomorfismo de Frobenius $\varphi$ possui a propriedade de satisfazer a equação característica $\alpha^{2}-c \alpha+q=0$.

\subsection{Multiplicação complexa (CM)}

Toda curva elíptica definida sobre um corpo finito vem equipada com um conjunto de operações que pode ser visualizada como uma multiplicação por inteiros na álgebra complexa [1]. Essas operações podem ser usadas por certas famílias de curvas para aumentar a eficiência da multiplicação elíptica.

Considere o endomorfismo de Frobenius $\varphi$ (o mesmo vale para qualquer endomofismo $\phi$ de forma análoga). Devido ao fato do mesmo satisfazer a

\footnotetext{
${ }^{1}$ ver essa definição no âpendice A
} 
$\alpha^{2}-c \alpha+q=0$, pode-se dizer que $\alpha=\left(c+\sqrt{c^{2}-4 q}\right) / 2$. Se, por exemplo, $q=2$ e $c=1$, então $\alpha=(1+\sqrt{-7}) / 2$.

Existe um homomorfimo natural do anel $\mathbb{Z}[\alpha]=\{a+b \alpha \mid a, b \in \mathbb{Z}\} \subset \mathbb{C}$ para o anel endomórfico de $E(\operatorname{End}(E))$ que mapeia $\alpha$ para $\varphi$. Portanto se existe uma expansão $K=\sum_{j} k_{j} \alpha^{j}$ em $\mathbb{Z}[\alpha]$ imediatamente obtém-se uma expansão $K=\sum_{j} k_{j} \varphi^{j}$ em $\operatorname{End}(E)$, tal que $K P=\sum_{j} k_{j} \varphi^{j}(P)$.

\subsection{Curva anômala}

Segundo Koblitz [1] uma curva elíptica $E$ definida sobre $\mathbb{F}_{q}$ é anômala se o módulo do traço do endomorfismo de Frobenius for 1 . Como $\# E\left(\mathbb{F}_{q}\right)=$ $q+1-c$, temos que $\# E\left(\mathbb{F}_{q}\right)=q \quad$ (para $\left.c=1\right)$. Nesta curva anômala o endomorfismo de Frobenius $\varphi$ satisfaz a equação característica $\alpha^{2}-\alpha+q=0$.

É de interesse o twist ${ }^{2} \tilde{E}$ de $E$, cujo endomorfismo de Frobenius satisfaz a equação característica $\alpha^{2}+\alpha+q=0$. $\tilde{E}_{n}$ simboliza o twist da curva $E$ considerada sobre o corpo estendido $\mathbb{F}_{q^{n}}$. Quando $q$ é ímpar e $E$ possuir equação $y^{2}=x^{3}+a x+b, \quad \tilde{E}_{n}$ tem equação $\beta y^{2}=x^{3}+a x+b$, onde $\beta \in \mathbb{F}_{q^{n}}$ não é uma potência de 2 . Por outro lado, quando $q$ é par, as equações são como as descritas nos exemplos da seção 4.4 . Se $n=2^{r} n_{0}$ com $n_{0}$ ímpar, então $\tilde{E}_{n}$ pode ser definida sobre uma pequena extensão $\mathbb{F}_{q^{2^{r}}}$, e o mapeamento de Frobenius $(x, y) \rightarrow\left(x^{q^{q^{r}}}, y^{q^{2^{r}}}\right)$ satisfaz a equação $\alpha^{2}+c \alpha+q^{2^{r}}=0$, onde $c$ é o traço do número complexo $((1+\sqrt{1-4 q}) / 2)^{2^{r}}$.

\subsection{Multiplicação em curvas elípticas anômalas de característica 2}

O caso mais importante na prática é aquele em que $q=2^{r}$. Neste caso a computação de $\varphi:(x, y) \rightarrow\left(x^{2^{r}}, y^{2^{r}}\right)$ sobre um ponto $\mathbb{F}_{2^{r n}}$ da curva elíptica representado na base normal ${ }^{3}$ é obtido através de uma operação de deslocamento de tempo desprezível. Portanto, para multiplicar um ponto $P$ por $2^{r}$ em uma curva anômala, o caminho mais rápido é usar a identidade $\alpha^{2}-\alpha+2^{r}=0$, isto é, $2^{r} P=\varphi(P)-\varphi^{2}(P)$, desde que ao invés de $r$ adições de pontos (método binário) é necessário executar apenas uma. (Na curva $\tilde{E}$, tem-se $2^{r} P=-\varphi(P)-\varphi^{2}(P)$.)

Exemplo 4.1. Em $2^{4}=16=\langle 10000\rangle_{2}$, o método binário exige 4 adições de pontos.

A maior eficiência com este método foi conseguido por Menezes e Vanstone []], que estavam trabalhando sobre curvas elípticas definidas sobre $\mathbb{F}_{2}$

\footnotetext{
${ }^{2}$ Se em $E$ o valor do traço de Frobenius possui valor igual a $c$, então no seu twist $\tilde{E}$ o seu valor é igual a $-c$. Além disso, $E$ e $\tilde{E}$ são isomorfas.

${ }^{3}$ ver definição no apendice $\mathrm{A}$
} 
e com traço do endomorfismo de Frobenius igual a $0(c=0)$, isto é, satisfazendo a equação $\alpha^{2}+2=0$. Neste caso, desde que $2 P=-\varphi^{2}(P)$, nenhuma adição de pontos é necessária, isto é, a duplicação de um ponto não possui custo computacional. Contudo, curvas $\operatorname{com} c=0$ são supersingulares. Em [1:] foi mostrado que o problema do logaritmo discreto em curvas elípticas supersingulares reduz-se ao problema do logaritmo discreto em um grupo multiplicativo de um corpo finito de mais ou menos o mesmo tamanho. Isto é, criptosistemas baseados em curvas elípticas supersingulares não são mais seguros do que os criptosistemas originais Diffie-Hellman.

Teorema 4.1. Seja E uma curva elíptica anômala definida sobre $\mathbb{F}_{q}$, e seja $\tilde{E}$ o seu twist.

(a) Se $P$ é um ponto $\mathbb{F}_{q^{n}}$ sobre $E$ (ou Ẽ), então a múltiplicação $q P$ pode ser computada com uma única adição de pontos (junto com operações de deslocamento para a computação de $x \rightarrow x^{q}$ em uma base normal de $\mathbb{F}_{q^{n}}$ ).

(b) No caso especial em que $q=2$, qualquer dos múltiplos $2^{l} P$ para $l \leq 4$, ou seja, $2 P, 4 P, 8 P$ e $16 P$, pode ser computada com uma adição simples de pontos.

Demonstração. A parte (a) segue da discussão acima, ou seja, como $\alpha^{2}-$ $\alpha+q=0$, então $q P=\varphi(P)-\varphi^{2}(P)$.

No caso (b), à primeira vista pode parecer que utilizar uma curva anômala não possui nenhuma vantagem pois computar $2 P=P+P$ custa também uma única adição de pontos. Contudo, ao se usar a relação $\alpha-\alpha^{2}=2$ satisfeita por $\varphi$ (que é definida para os pontos $\mathbb{F}_{2^{n}}$ de $E$ por $(x, y) \rightarrow\left(x^{2}, y^{2}\right)$ ), temos que:

$$
\begin{aligned}
4 & =2 \cdot 2=2 \alpha-2 \alpha^{2}=\left(\alpha-\alpha^{2}\right) \alpha-2 \alpha^{2}=-\alpha^{3}-\alpha^{2} \\
8 & =4 \cdot 2=\left(-\alpha^{3}-\alpha^{2}\right)\left(\alpha-\alpha^{2}\right)=-\alpha^{3}+\alpha^{5} \\
16 & =4^{2}=\alpha^{6}+2 \alpha^{5}+\alpha^{4}=\alpha^{6}+\left(\alpha-\alpha^{2}\right) \alpha^{5}+\alpha^{4} \\
& =-\alpha^{7}+2 \alpha^{6}+\alpha^{4}=-\alpha^{7}+\left(\alpha-\alpha^{2}\right) \alpha^{6}+\alpha^{4}=\alpha^{4}-\alpha^{8}
\end{aligned}
$$

(Fórmulas análogas em $\tilde{E}$ são obtidas substituindo-se $\alpha$ por $-\alpha$, pois nesse caso $\alpha=(-1+\sqrt{-7}) / 2$.)

Portanto, na computação de $K P$, qualquer string com menos de 4 zeros pode ser tratada com uma única adição de pontos.

\subsubsection{Números de Pontos}

Seja $E\left(\mathbb{F}_{q}\right)$ uma curva elíptica anômala definida sobre $\mathbb{F}_{q}$ e $\tilde{E}\left(\mathbb{F}_{q}\right)$ o seu twist. Existe uma relação entre o número de pontos da curva $E\left(\mathbb{F}_{q}\right)$ (e também $\tilde{E}\left(\mathbb{F}_{q}\right)$ ) considerada sobre o corpo estendido $\mathbb{F}_{q^{n}}$ e a raiz $\alpha=$ $(1+\sqrt{1-4 q}) / 2$ do polinômio característico $\alpha^{2}-\alpha+q=0$, ou seja, $\mathcal{N}_{n}=$ 
$\left|\alpha^{n}-1\right|^{2}, \tilde{\mathcal{N}}_{n}=\left|\alpha^{n}+1\right|^{2}$. Isto conduz a um algoritmo para computar $\mathcal{N}_{n} \mathrm{e}$ $\tilde{\mathcal{N}}_{n}$ (ver Teorema 2.2):

$$
\begin{aligned}
& \mathcal{N}_{n}=q^{n}+1-a_{n} \\
& \tilde{\mathcal{N}}_{n}=q^{n}+1+a_{n} \quad \text { onde } a_{n+1}=a_{n}-q a_{n-1} \text { para } n \geq 1 .
\end{aligned}
$$

Deseja-se encontrar um corpo estendido $\mathbb{F}_{q^{n}}$ tal que o número $\mathcal{N}_{n}$ de pontos $\mathbb{F}_{q^{n}}$ sobre $E\left(\mathbb{F}_{q}\right)$ ou o número $\tilde{\mathcal{N}}_{n}$ de pontos $\mathbb{F}_{q^{n}}$ sobre $\tilde{E}\left(\mathbb{F}_{q}\right)$ seja múltiplo de um número primo grande (de no mínimo 30 dígitos) (ver aspectos relativos a segurança na seção 2.9 ).

Como $\mathcal{N}_{n}=\left|\alpha^{n}-1\right|^{2}$ e $\tilde{\mathcal{N}}_{n}=\left|\alpha^{n}+1\right|^{2}$, segue que $\mathcal{N}_{n_{1}} \mid \mathcal{N}_{n}$ quando $n_{1} \mid n$ e que $\tilde{\mathcal{N}}_{n_{1}} \mid \tilde{\mathcal{N}}_{n}$ quando $n / n_{1}$ for um inteiro ímpar. Tal que se $\mathcal{N}_{n}$ (respectivamente $\tilde{\mathcal{N}}_{n}$ ) é o produto de um pequeno fator e um número primo grande, devemos tomar $n$ como um primo (respectivamente igual a um número primo ou senão um número primo vezes uma pequena potência de 2).

Exemplo 4.2. $\operatorname{Em} E\left(\mathbb{F}_{q}\right)$, se $n_{1}=1,1 \mid n$ para qualquer $n$ primo. Portanto, como $\mathcal{N}_{1}=q$, temos que $\mathcal{N}_{n}=q \cdot$ primo. $\operatorname{Em} \tilde{E}\left(\mathbb{F}_{q}\right)$, se $n_{1}=1,1 \mid n$ para qualquer $n$ primo ou $n$ igual a um primo vezes uma pequena potência de 2 . Portanto, como $\tilde{\mathcal{N}}_{1}=q+2$, temos que $\tilde{\mathcal{N}}=(q+2) \cdot$ primo.

Mostraremos a seguir exemplos de curvas definidas em corpos de característica 2 que são anômalas. Isto pode ser verificado através do uso do MAGMA [1] (ver implementação no Apendice B).

\subsubsection{Exemplos definidos em $\mathbb{F}_{2}$}

Considere a curva anômala $E: y^{2}+x y=x^{3}+x^{2}+1$ sobre $\mathbb{F}_{2}$ e seu twist $\tilde{E}: y^{2}+x y=x^{3}+1$ que possui multiplicação complexa por $Q(\sqrt{-7})(\Delta=$ $1-4 \cdot 1 \cdot 1=-7$ ). ${ }^{4}$ Para um certo primo $n$ tem-se $\mathcal{N}_{n}=2 \cdot$ primo (ou $\tilde{\mathcal{N}}_{n}=4 \cdot$ primo). Foram listados todos os valores de $\mathcal{N}_{n} / 2$ e $\tilde{\mathcal{N}}_{n} / 4$ para $n<200{ }^{5}$ que são primos (segundo o autor primos prováveis, desde que foi verificado a primalidade usando Matemática) e que possuem no mínimo 30

\footnotetext{
${ }^{4} Q(\sqrt{\Delta})$ é um corpo imaginário quadrático, onde $\Delta$ é nesse caso o discriminante da equação característica da curva.

${ }^{5}$ Esses valores podem ser conferidos com o uso do software MAGMA | |
} 
dígitos.

$$
\begin{aligned}
& \mathcal{N}_{101} / 2=1267650600228230886142808508011 \\
& \mathcal{N}_{107} / 2=81129638414606692182851032212511 \\
& \mathcal{N}_{109} / 2=324518553658426701487448656461467 \\
& \mathcal{N}_{113} / 2=5192296858434827627896703833467507 \\
& \mathcal{N}_{163} / 2=5846006549323611672814741753598448348329118574063 \\
& \tilde{\mathcal{N}}_{103} / 4=2535301200456459535862530067069 \\
& \tilde{\mathcal{N}}_{107} / 4=40564819207303335604363489037809 \\
& \tilde{\mathcal{N}}_{131} / 4=680564733841876926932320129493409985129
\end{aligned}
$$

Portanto, por exemplo, o número de pontos na curva $y^{2}+x y=x^{3}+1$ sobre $\mathbb{F}_{2^{131}}$ (um corpo que de acordo com a tabela em [1!] possui uma base normal ótima) é divisível por primo provável de 39 dígitos. (O corpo $\mathbb{F}_{2^{113}}$ também possui base normal ótima.)

\subsubsection{Exemplos definidos em $\mathbb{F}_{4}$}

Considere a curva $E: y^{2}+x y=x^{3}+\gamma$, onde $\gamma \in \mathbb{F}_{4}$ satisfaz $\gamma^{2}=\gamma+1$, e seu twist $\tilde{E}: y^{2}+x y=x^{3}+\gamma x^{2}+\gamma$ que possuem multiplicação complexa. por $Q(\sqrt{-15})(\Delta=1-4 \cdot 1 \cdot 4=-15)$. Para um certo primo $n$ tem-se $\mathcal{N}_{n}=4 \cdot \operatorname{primo}\left(\right.$ ou $\tilde{\mathcal{N}}_{n}=6$. primo). Foram listados todos os valores de $\mathcal{N}_{n} / 4$ e $\tilde{\mathcal{N}}_{n} / 6$ para $n<100$ :

$$
\begin{aligned}
& \mathcal{N}_{67} / 4=5444517870735015415344659586094410599059 \\
& \mathcal{N}_{79} / 4=91343852333181432387730573045979447452365303319 \\
& \tilde{\mathcal{N}}_{59} / 6=55384499824371494566505749990887769
\end{aligned}
$$

Os corpos $\mathbb{F}_{44^{67}}$ e $\mathbb{F}_{4^{79}}$ possuem base normal ótima [1:].

\subsubsection{Exemplos definidos em $\mathbb{F}_{8}$}

Considere a curva $E: y^{2}+x y=x^{3}+\gamma$, onde $\gamma \in \mathbb{F}_{8}$ satisfaz $\gamma^{3}=\gamma+1$, e seu twist $\tilde{E}: y^{2}+x y=x^{3}+x^{2}+\gamma$ que possuem multiplicação complexa por $Q(\sqrt{-31})(\Delta=1-4 \cdot 1 \cdot 8=-31)$. Para um certo primo $n$ tem-se $\mathcal{N}_{n}=8 \cdot$ primo (ou $\tilde{\mathcal{N}}_{n}=10 \cdot$ primo). Foram listados todos os valores de $\mathcal{N}_{n} / 8$ e $\tilde{\mathcal{N}}_{n} / 10$ para $n<66$ :

$$
\begin{aligned}
\mathcal{N}_{37} / 8 & =324518553658426732114957572335741 \\
\tilde{\mathcal{N}}_{47} / 10 & =278759314981632789268903181396173621874561 \\
\tilde{\mathcal{N}}_{59} / 10 & =19156194260823610729479337915747318375048137080701777
\end{aligned}
$$




\subsubsection{Exemplos definidos em $\mathbb{F}_{16}$}

Finalmente, foi considerado $\tilde{E}=y^{2}+x y=x^{3}+\gamma x^{2}+1$ de $E$, onde $\gamma \in \mathbb{F}_{16}$ é um elemento com traço absoluto igual a 1, que é uma curva anômala, pelo fato da quarta potência de $\alpha=(1+\sqrt{-7}) / 2$ ser $(1-3 \sqrt{-7}) / 2$. Para um certo $n$ igual a quatro vezes um número primo, tem-se que $\tilde{\mathcal{N}}_{n}=\tilde{\mathcal{N}}_{4} \cdot$ primo $=$ 18 primo. Existe um caso para $n<200$ quando $\tilde{\mathcal{N}}_{n} / 18$ é um primo de mais do que 30 dígitos.

$$
\tilde{\mathcal{N}}_{148} / 18=19822884620916109459140767798279811163792081
$$

$\mathrm{O}$ corpo $\mathbb{F}_{2^{148}}$ possue base normal ótima [?].

\subsubsection{Conclusão}

Todas as curvas elípticas acima promovem os criptosistemas do tipo DiffieHellman seguros.

Os exemplos da Seção 4.4.2 tem a característica adicional que, quando computamos um múltiplo $K P$, qualquer string de número de zeros menor ou igual a 4 na representação binária de $K$ pode ser tratada com somente uma adição única de pontos.

No caso dos exemplos das Seções 4.4.3, 4.4.4 e 4.4 .5 uma string, respectivamente de 2, 3 e 4 zeros na representação binária de $K$ pode ser tratada com uma única adição de pontos (note que $q=\alpha-\alpha^{2}$ ).

A próxima seção tratará apenas dảs curvas definidas sobre $\mathbb{F}_{2}$.

\subsection{Alguns aspectos da implementação eficiente}

\subsubsection{Expansão binária balanceada}

Uma expansão binária balanceada (por exemplo a expansão NAF) de um inteiro $K$ possui uma quantidade de zeros maior do que sua expansão binária. De fato, na média $2 / 3$ dos dígitos de uma expansão binária balanceada são zeros. Portanto, ao se usar as curvas anômalas da Seção 4.42 (curvas definidas sobre $\mathbb{F}_{2}$ ), a rápida conversão de $K$ para a expansão binária balanceada habilitará computar $K P$ de maneira mais rápida, devido ao fato de que qualquer string de número de zeros menor ou igual a 4 poder ser tratada com uma única adição de pontos.

Exemplo 4.3. Computar $15 P=16 P-1$ requer somente 2 adições de pontos 


\subsubsection{Domínio Euclidiano e função norma}

O anel $\mathbb{Z}[\alpha]=\{a+b \alpha \mid a, b \in \mathbb{Z}\}$ é um domínio Euclidiano em relação a sua norma, ou seja, em relação a $N(a+b \alpha)=\|a+b \alpha\|^{2}=a^{2}+a b+2 b^{2} \quad 6$. Por ser um domínio Euclidiano, temos que para qualquer $K, t \in \mathbb{Z}[\alpha]$ com $t \neq 0$, existe $q, K^{\prime} \in \mathbb{Z}[\alpha]$ tal que $K=q t+K^{\prime}, \operatorname{com} N\left(K^{\prime}\right) \leq N(t)$.

\subsubsection{Expansão na base $\varphi$}

Considere que estejamos trabalhando em um grupo de pontos $\mathbb{F}_{2^{n}}$ de uma curva anômala definida em $\mathbb{F}_{2}: E: y^{2}+x y=x^{3}+x^{2}+1$ ou $\tilde{E}: y^{2}+x y=$ $x^{3}+1$.

Sobre $E$ o endomorfismo de Frobenius $\varphi:(x, y) \rightarrow\left(x^{2}, y^{2}\right)$ é o elemento $\alpha=(1+\sqrt{-7}) / 2$ do anel endomórfico $\mathbb{Z}[\alpha]=\{a+b \alpha \mid a, b \in \mathbb{Z}\}$ (em $\tilde{E}$ é $\alpha=(-1+\sqrt{-7}) / 2$ ). Assim sendo, múltiplos $K P$, onde $K \in \mathbb{Z}$ poderiam ser computados, através da representação de $K$ na base $\varphi$. Formalmente, desde que $\alpha=(1+\sqrt{-7}) / 2$ é um elemento de norma 2 no domínio Euclidiano $\mathbb{Z}[\alpha]$ qualquer elemento do anel, em particular $K$, possui representação única na forma $\sum k_{j} \alpha^{j}$, onde $k_{i} \in\{0,1\}$, de forma que $K P=\sum k_{j} \varphi^{j}(P)$.

\subsubsection{Expansão balanceada na base $\varphi$}

Pode-se obter uma expansão balanceada na base $\alpha$ de $K$. Note que $\varphi$ satisfaz $\alpha(1+\alpha)=2$ em $E($ e $-\alpha(1-\alpha)=2$ em $\tilde{E})$. Vamos trabalhar sobre $E$ (o argumento para $\tilde{E}$ é análogo com a expansão $\varphi$ substituída pela expansão $-\varphi)$.

Faça $K=K_{0}+2 K_{1}+2 K_{2}$, onde $K_{0} \in\{0,1\}$ e $K_{2}$ é parte da expansão $\alpha$ de $\left(K-K_{0}\right) / 2$ consistindo de todas as seqüências de $\geq 2$ bits 1 consecutivos. Como $2\left(1+\alpha+\alpha^{2}+\cdots+\alpha^{j-1}\right)=\alpha-\alpha^{j+1}$, substitui-se cada seqüência de $j \geq 2$ bits 1 consecutivos (11..11) na expansão de $K_{2}$ por $\overline{1} 0 \ldots 10$.

Exemplo 4.4. Vamos supor que $K=1+2+2 \alpha+2 \alpha^{2}$, portanto temos que $K=1+2\left(1+\alpha+\alpha^{2}\right)$ e podemos simplificar $K$ obtendo $K=1+\alpha-\alpha^{4}$.

Infelizmente expressar um arbitrário $K$ como uma expansão balanceada em $\alpha$ não será necessariamente mais eficiente do que usar uma expansão binária balanceada. Isto porque a expansão balanceada em $\alpha$ de $K$ conter normalmente, segundo o autor, duas vezes mais bits do que a sua expansão binária balanceada.

\footnotetext{
${ }^{6}$ Considerando o endomorfismo de Frobenius, temos que $\alpha=\frac{\left(c+\sqrt{c^{2}-4 q}\right)}{2}$. Assim sendo, $a+b \alpha=a+(c / 2) b+\left(\sqrt{4 q-c^{2}} / 2\right) b i$, de tal forma que $N(a+b \alpha)=(a+(c b / 2))^{2}+$ $(1 / 4)\left(4 q-c^{2}\right) b^{2}$. No caso em que $q=2$ e $c=1$, obtem-se $N(a+b \alpha)=a^{2}+a b+2 b^{2}$.
} 


\subsection{Conclusão}

Neste capítulo foi descrita em linhas gerais uma maneira de se usar o endomorfismo de Frobenius para acelerar a multiplicação em curvas elípticas anômalas definidas em pequenos corpos de característica $2\left(\mathbb{F}_{2}, \mathbb{F}_{4}, \mathbb{F}_{8}, \mathbb{F}_{16}\right)$, bem como apresentar exemplos dessas curvas.

Nossa contribuição foi detalhar o artigo [10] (básico para os propósitos desta dissertação), bem como destacar conceitos, tais como endomorfismo de Frobenius, multiplicação complexa, equação característica de uma curva elíptica, curva anômala, twist de uma curva, função norma, domínio Euclidiano. Mostramos também, no Apêndice B, como utilizar o software MAGMA [1] para verificar as propriedades da curvas apresentadas, tais como o traço do endomorfismo de Frobenius das mesmas. 


\section{Capítulo 5}

\section{Método de Meier e Staffelbach para multiplicação em curvas definidas sobre $\mathbb{F}_{2}$}

O uso de curvas elípticas definidas sobre corpos finitos em criptosistemas do tipo Diffie-Hellman foi proposto por Koblitz [1] .

O objetivo deste capítulo é descrever um algoritmo apresentado por Meier e Staffelbach [] que é três vezes mais rápido do que o método binário, é fácil de implementar e não é baseado na pré-computação ou memória adicional.

Da mesma forma que Koblitz, os autores concentraram seus esforços na curva anômala definida sobre $\mathbb{F}_{2}$

$$
E: y^{2}+x y=x^{3}+x^{2}+1
$$

e o seu twist $\tilde{E}: y^{2}+x y=x^{3}+1$. Essas curvas serão consideradas sobre o corpo estendido $\mathbb{F}_{2^{n}}$. Portanto, tomemos $E_{n}$ como que denotando os pontos $\mathbb{F}_{2^{n}}$ sobre a curva $E$ e $\tilde{E}_{n}$ os pontos $\mathbb{F}_{2^{n}}$ sobre a curva $\tilde{E}$.

Koblitz sugeriu expressar multiplicações por $K$ como uma combinação linear de potências do mapeamento de Frobenius $(\varphi)$ devido ao fato de que os $\varphi^{j}$ podem ser facilmente computados através de elevações ao quadrado no corpo estendido $\mathbb{F}_{2^{n}}$ quando o mesmo é representado na base normal (ver apentice A). Expansões da forma

$$
K=\sum_{j} k_{j} \alpha^{j}
$$

são consideradas com $k_{j} \in\{0, \pm 1\}$. Com esta representação de $K$ a computação de $K P$ pode ser reduzida a $\left(l_{a}-1\right)$ adições, onde $l_{a}$ é o número de termos diferentes de zero na expansão de $K$. No entanto, as expansões obtidas por Koblitz são em média duas vezes o comprimento da expansão binária de $K$. 
Neste capítulo descreve-se a construção de expansões curtas da equação 5.2 . Foi provado que sempre existe uma expansão $K=\sum_{j=0}^{n-1} k_{j} \alpha^{j}$ de comprimento $n$, onde $n$ é o grau de extensão do corpo $\mathbb{F}_{2^{n}}$ (Teorema 5.1). A demonstração do Teorema 5.1 conduz a um algoritmo eficiente que produz expansões em que se espera metade dos coeficientes $k_{j}$ iguais a zero (Corolário 5.1).

Desde que $\varphi^{j}$ é obtido quase sem custo, a expansão em $\varphi$ de $K$ permite computar $K P$ de um arbitrário ponto $P$ em $E_{n} \operatorname{com} n / 2$ adições na média que é uma melhoria de fator 3 comparado ao método binário sem o uso de pré-computação ou memória adicional ${ }^{1}$.

Teorema 5.1. Seja a curva anômala $E: y^{2}+x y=x^{3}+x^{2}+1$ definida sobre $\mathbb{F}_{2}$ e consideremos $E_{n}$ como o grupo de pontos $\mathbb{F}_{2^{n}}$ sobre $E$. Então em $E_{n}$ a multiplicação por um inteiro $K$ pode ser expresso como

$$
K=\sum_{j=0}^{n-1} k_{j} \varphi^{j}
$$

com $k_{j} \in\{0, \pm 1\}$.

Este teorema se mantém para $\tilde{E}_{n}$. A prova se processa em muitos passos.

Primeiro deve-se observar que o endomorfismo de Frobenius satisfaz a equação $\alpha^{2}-\alpha+2=0$, e que existe um homomorfismo natural do anel $\mathbb{Z}[\alpha]=\{a+b \alpha \mid a, b \in \mathbb{Z}\} \subset \mathbb{C}$ para o anel endomórfico de $E(\operatorname{End}(E))$ que mapeia $\alpha=1+\sqrt{-7} / 2$ para $\varphi$. Portanto, se existe uma expansão $K=\sum_{j} k_{j} \alpha^{j}$ em $\mathbb{Z}[\alpha]$, temos uma expansão correspondente $K=\sum_{j} k_{j} \varphi^{j}$ em $\operatorname{End}(E)$, o que significa que $K P=\sum_{j} k_{j} \varphi^{j}(P)$ para todo ponto $P \mathrm{em}$ $E_{n}$ (ver Seção 4.2).

Para encontrar tal expansão em $\mathbb{Z}[\alpha]$ deve-se fazer o uso da estrutura algébrica do anel $\mathbb{Z}[\alpha]$, que é um domínio Euclidiano em relação a norma $N(a+b \alpha)=|a+b \alpha|^{2}=(a+b \alpha)(a+b \bar{\alpha})=a^{2}+a b+2 b^{2}$ (ver Seção 4.5.2).

Lema 5.1. Para qualquer $s, t \in \mathbb{Z}[\alpha]$, com $t \neq 0$, existe $q, r \in \mathbb{Z}[\alpha]$ tal que $s=q t+r \operatorname{com} N(r) \leq 4 / 7 N(t)$.

Demonstração. Os elementos do anel $\mathbb{Z}[\alpha]$ formam um reticulado em $\mathbb{C}$, e a totalidade de $\mathbb{C}$ pode ser envolvida por triângulos cujos vértices estão em $\mathbb{Z}[\alpha]$, como descrito na Figura 5.1. Considere o triângulo com vértices 0,1 e $\alpha$. O ponto $\tau=1 / 2+(3 / 2 \sqrt{7}) i$ é o centro do círculo circunscrito do triângulo, como é facilmente verificável computando as distâncias de $\tau$ para cada vértice, que é $|\tau-0|=|\tau-1|=|\tau-\alpha|=2 / \sqrt{7}$. Portanto qualquer outro ponto no triângulo possui distância menor do que $2 / \sqrt{7}$ para algum vértice.

\footnotetext{
${ }^{1}$ No método binário o custo médio é de $m$ duplicaçōes mais $m / 2$ adições, $o$ que dá um total $3 m / 2$ adiçōes onde $m=\log _{2} \eta \leq \log _{2} \mathcal{N}_{n}=\log _{2} 2^{n}=n$ (ver Seção 3. i).
} 


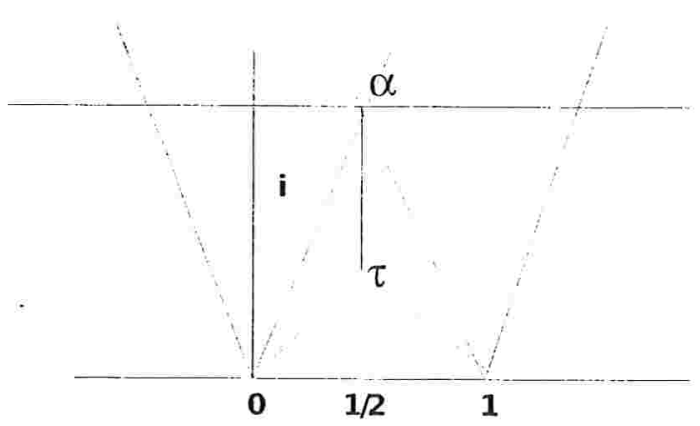

Figura 5.1: O reticulado $\mathbb{Z}[\alpha]$

Desde que qualquer ponto $z \in \mathbb{Z}[\alpha]$ repousa em algum triângulo, concluímos que para qualquer número complexo $z \in \mathbb{C}$ existe um elemento $u \in \mathbb{Z}[\alpha]$ $\operatorname{com} N(z-u) \leq(2 / \sqrt{7})^{2}=4 / 7$.

Sejam $s, t \neq 0 \in \mathbb{Z}[\alpha]$ e considere o quociente $v=s / t$ computado no corpo quociente de $\mathbb{Z}[\alpha]$, isto é, no corpo $\mathbb{Q}(\alpha)=\{a+b \alpha \mid a, b \in \mathbb{Q}\} \subset \mathbb{C}$. Como discutido anteriormente, existe um elemento $q \in \mathbb{Z}[\alpha]$ onde $N(v-q) \leq 4 / 7$. Como $r=s-q t=v t-q t=t(v-q)$, então $N(r)=N(v-q) N(t) \leq 4 / 7 N(t)$, e portanto $q, r \in \mathbb{Z}[\alpha]$ obedecem as propriedades do Lema 5.1 .

Lema 5.2. Para qualquer $s \in \mathbb{Z}[\alpha]$ com norma $N(s)<2^{n}, n \in \mathbb{N}$, existe uma expansão $s=\sum_{j=0}^{n-1} k_{j} \alpha^{j}$ de comprimento $n$ com $k_{j} \in\{0, \pm 1\}$.

Demonstração. A prova é por indução em $n$.

Para $n=1,2$, considere os elementos $s \in \mathbb{Z}[\alpha] \operatorname{com} N(s)<4$. São eles: $s=0$ (norma 0$), s= \pm 1$ (norma 1$), s= \pm \alpha$ (norma 2) e $s= \pm(1-\alpha)$ (norma 2). Para estes elementos a afirmação do Lema 5.2 se mantém, como pode ser visto através de uma inspeção direta.

Agora considere $s \in \mathbb{Z}[\alpha]$ com $N(s)<2^{n}, n>2$. Desde que $\mathbb{Z}[\alpha]$ é um domínio Euclidiano (ver Seção 4.5.2), s pode ser expresso como

$$
s=s^{\prime} \alpha+k
$$

onde $N(k)<N(\alpha)=2$, isto é, com $k \in\{0, \pm 1\}$. A idéia é reduzir o problema de encontrar uma expansão para $s$ ao problema de encontrar uma expansão para $s^{\prime}$. Se $k=0$, isto é, se $\alpha$ divide $s$, a redução (5.4) é única. Caso contrário, como $N(\alpha)=2$, sempre existe uma redução com $k=1 \mathrm{e}$ outra com $k=-1$.

Se a redução pudesse ser feita tal que $N\left(s^{\prime}\right) \leq N(s) / 2<2^{n-1}$, a prova seria facilmente completada por indução. No entanto, existem situações onde não existe nenhuma redução com $N\left(s^{\prime}\right) \leq N(s) / 2$, como veremos abaixo. Faremos distinção em 3 casos:

Caso não-crítico existe uma redução (5.4) $\operatorname{com} N\left(s^{\prime}\right)<N(s) / 2$; 


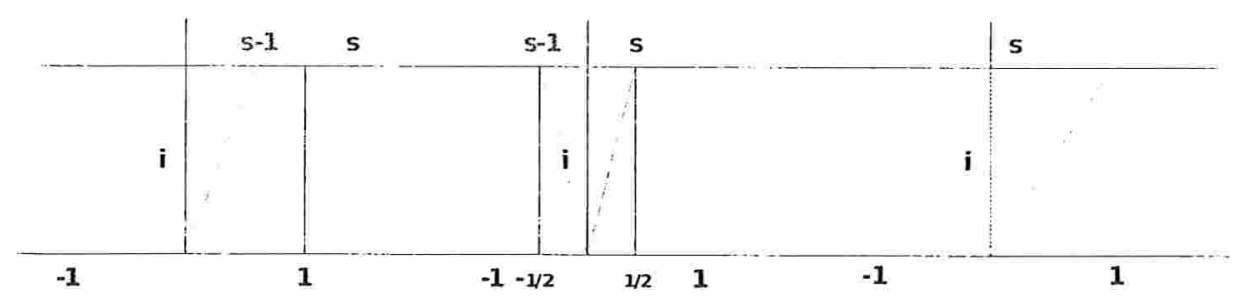

Figura 5.2: Casos não-crítico, semi-crítico e crítico.

Caso semi-crítico existe uma redução (5.4) $\operatorname{com} N\left(s^{\prime}\right)=N(s) / 2$;

Caso crítico existem somente reduções (5.4) com $N\left(s^{\prime}\right)>N(s) / 2$.

Se $\alpha$ divide $s$, temos a redução $s=s^{\prime} \alpha \operatorname{com} k=0$ e $N\left(s^{\prime}\right)=N(s) / 2$, isto é, $s$ é semi-crítico. Se $\alpha$ não divide $s$, então $\alpha$ divide tanto $s-1$ quanto $s+1$. Neste caso o tipo de redução depende do valor absoluto da parte real $\mathbb{R}(s)$ de $s^{2}$.

Caso não-crítico: $|\mathbb{R}(s)| \geq 1$.

Assuma que $\mathbb{R}(s) \geq 1$, como ilustrado na Figura 5.2. Então $N(s-$ 1) $<N(s)$, e temos a redução $s=s^{\prime} \alpha+1(s-1$ é divisível por $\alpha) \mathrm{com}$ $N\left(s^{\prime}\right)=N(s-1) / N(\alpha)<N(s) / 2$.

Similarmente, se $\mathbb{R}(s) \leq-1$, então $N(s+1)<N(s)$ e temos a redução $s=s^{\prime} \alpha-1(s+1$ é divisívèl por $\alpha) \operatorname{com} N\left(s^{\prime}\right)=N(s+1) / N(\alpha)<N(s) / 2$.

Caso semi-crítico: $|\mathbb{R}(s)|=1 / 2$

Assuma que $\mathbb{R}(s)=1 / 2$, como ilustrado na Figura 5.2. Então $N(s-1)=$ $N(s)$ (veja figura). Supondo agora $s$ não divisível por $\alpha$, temos a redução $s=s^{\prime} \alpha+1(s-1$ é divisível por $\alpha)$ com $N\left(s^{\prime}\right)=N(s-1) / N(\alpha)=N(s) / 2$ ( $\log s$ é divisível por $\alpha$ ).

Similarmente, se $\mathbb{R}(s)=-1 / 2$, então $N(s+1)=N(s)$ e temos a redução $s=s^{\prime} \alpha-1 \operatorname{com} N\left(s^{\prime}\right)=N(s+1) / N(\alpha)=N(s) / 2$.

Caso crítico: $\mathbb{R}(s)=0$

Pelo teorema de Pitágoras, $N(s-1)=N(s+1)=N(s)+1$ e temos a redução $s=s^{\prime} \alpha+1$ ( $s-1$ é divisível por $\left.\alpha\right)$ e $s=s^{\prime \prime} \alpha-1(s+1$ é divisível por $\alpha$ ) com

$$
N\left(s^{\prime}\right)=N\left(s^{\prime \prime}\right)=N(s-1) / N(\alpha)=N(s+1) / N(\alpha)=(N(s)+1) / 2
$$

Desde que $\left(s^{\prime \prime}-s^{\prime}\right)=2 / \alpha=1-\alpha, s^{\prime}$ ou $s^{\prime \prime}$ não é divisível por $\alpha$.

\footnotetext{
${ }^{2} s=a+b \alpha=a+(1 / 2) b+(\sqrt{-7} / 2) b$ e portanto $\mathbb{R}(s)=a+(1 / 2) b$.
} 
Se assumirmos que $s^{\prime}$ não seja divisível por $\alpha$, prova-se que $s^{\prime}$ possui uma redução não-crítica $s^{\prime}=s^{\prime \prime \prime} \alpha+k^{\prime}$, tal que $N\left(s^{\prime \prime \prime}\right)<N\left(s^{\prime}\right) / 2$. Para isso é suficiente mostrar que $\left|\mathbb{R}\left(s^{\prime}\right)\right| \geq 1$.

Desde que $\mathbb{R}(s)=0, s$ deve ser da forma $s=a \sqrt{-7}$ para algum inteiro ímpar $a \in \mathbb{Z} .^{3}$

Então $s^{\prime}$ pode ser computado em $\mathbb{Q}(\alpha)$ como:

$$
\begin{aligned}
s^{\prime} & =(s-1) \alpha^{-1}=(a \sqrt{-7}-1) \frac{1}{4}(1-\sqrt{-7}) \\
& =\frac{7 a-1}{4}+\frac{(1-a)}{4} \sqrt{-7}
\end{aligned}
$$

Segue que $\left|\mathbb{R}\left(s^{\prime}\right)\right|>3 / 2$ e portanto $s^{\prime}$ possui uma redução não-crítica, ou seja $N\left(s^{\prime \prime \prime}\right)<N\left(s^{\prime}\right) / 2$. Similarmente, $s^{\prime \prime}$ é não-crítico se $\alpha$ não divide $s^{\prime \prime}$.

Agora a prova do lema pode ser facilmente obtida.

No caso em que $s$ tem uma redução não-crítica ou semi-crítica $s=s^{\prime} \alpha+k$, tem-se $N\left(s^{\prime}\right) \leq N(s) / 2<2^{n-1}$. Por hipótese de indução, $s^{\prime}$ possui uma expansão em $\alpha$ de comprimento $n-1$, o que implica em uma expansão de $s$ em $\alpha$ de comprimento $n$.

No caso em que $s$ possui uma redução crítica $s=s^{\prime} \alpha+k$, tem-se, de acordo com a equação $5.5, N\left(s^{\prime}\right)=(N(s)+1) / 2 \leq 2^{n-1}$. Desde que a desigualdade $N\left(s^{\prime}\right) \leq 2^{n-1}$ não se mantém estritamente, não se pode aplicar a hipótese indutiva para $s^{\prime}$.

Contudo, como discutido acima, a redução pode ser feita tal que $s^{\prime}$ possua uma redução não-crítica $s^{\prime}=s^{\prime \prime \prime} \alpha+k^{\prime}$, isto é, $N\left(s^{\prime \prime \prime}\right)<N\left(s^{\prime}\right) / 2 \leq 2^{n-2}$. Portanto $s=s^{\prime} \alpha^{2}+k^{\prime} \alpha+k$, e por hipótese indutiva $s^{\prime \prime \prime}$ possui uma expansão em $\alpha$ de comprimento $n-2$ o que implica uma expansão de $s$ em $\alpha$ de comprimento $n$, completando a prova do lema.

Agora estamos em condições de provar o Teorema 5.1.

Demonstração do Teorema 5.1. Como $E_{n}$ é considerado sobre o corpo estendido $\mathbb{F}_{2^{n}}$, o mapeamento de Frobenius satisfaz a equação $\varphi^{n}(P)=P^{4}$ o que implica que $\alpha^{n}=1$. Segue que quaisquer duas expansões em $\alpha$ que sejam congruentes módulo $\alpha^{n}-1$, suas correspondentes expansões $\varphi$ correspondem o mesmo endomorfismo sobre $E_{n}$. Portanto computa-se a expansão $\alpha$ do resto $K^{\prime}$ da divisão de $K$ por $\alpha^{n}-1^{5}$ :

$$
K=q\left(\alpha^{n}-1\right)+K^{\prime}
$$

onde, de acordo com o Lema $5.1, N\left(K^{\prime}\right) \leq(4 / 7) N\left(\alpha^{n}-1\right)$.

\footnotetext{
${ }^{3} \mathbb{R}(s)=a+(1 / 2) b=0, \log 0 b=-2 a$. Como $s=a+(1 / 2) b+(\sqrt{-7} / 2) b$, temos que $s=-a \sqrt{-7}$. Se $a$ fosse par, $\alpha$ dividiria $s$ pois teríamos $a=2 \gamma, \gamma \in \mathbb{Z}$ e portanto, $N(s)=a^{2}+a b+2 b^{2}=a^{2}-2 a^{2}+8 a^{2}=7 a^{2}=28 \gamma^{2}$, que é sempre divisivel por $N(\alpha)=2$.

${ }^{4} \mathbb{F}_{2^{n}}$ possui $2^{n}$ elementos, portanto $x^{2^{n}}=x$ e $y^{2^{n}}=y$

${ }^{5} \mathrm{Na}$ seção 6.3 será explicado o algoritmo que obtém essa redução
} 
Contudo, para se obter um limite em $N\left(K^{\prime}\right)$ observa-se que

$$
N\left(\alpha^{n}-1\right)=q^{n}+1-\left(\alpha^{n}+\beta^{n}\right)=2^{n}+1-\left(\alpha^{n}+\beta^{n}\right)=\mathcal{N}_{n}
$$

onde $\alpha$ e $\beta$ em são raízes da equação característica $\alpha^{2}-\alpha+q=0$.

Pelo Teorema de Hasse (Teorema 2.1), $\mathcal{N}_{n}<f(n)=2^{n}+1+2^{\frac{n}{2}+1}$, e para $n \geq 4,(4 / 7) f(n)<2^{n} ; g(n)=2^{n}-(4 / 7) f(n)$ é estritamente crescente para $n \geq 1$ e estritamente positivo para $n=4$. Portanto, para $n \geq 4, N\left(K^{\prime}\right)<2^{n}$ e a prova do Teorema 5.1 segue do Lema 5.1, ou seja, $K=\sum_{j=0}^{n-1} k_{j} \varphi^{j}$ com $k_{j} \in\{0, \pm 1\}$.

Para $n \leq 3$ a afirmação do teorema pode ser verificada diretamente.

Corolário 5.1. $N a$ expansão $K=\sum_{j=0}^{n-1} k_{j} \varphi^{j}$ espera-se que metade dos coeficientes $k_{j}$ sejam iguais a zero.

Note que um elemento arbitrário $s=a+b \alpha$ em $\mathbb{Z}[\alpha]$ é divisível por $\alpha$ se e somente se $a$ é par. Portanto com probabilidade $1 / 2$ este elemento possui uma redução da forma $s=s^{\prime} \alpha$, isto é, com $k=0$. Continuando a redução, é de se esperar que resultados intermediários também possuam esta propriedade. Isto implicaria que metade dos coeficientes $k_{j}$ na expansão possam ser iguais a zero.

Note também que se $s$ é divisível por $\alpha$ então $s=a+b \alpha=s^{\prime} \alpha=$ $(A+B \alpha) \alpha$. Como $\alpha^{2}=\alpha-2$, temos que $a+b \alpha=A \alpha+B \alpha-2 B$. Portanto temos que $B=-a / 2$ e $A=b+a / 2$, ou seja, $a$ deve ser par. 


\subsection{Algoritmo para computar a expansão balanceada na base $\alpha$}

É fácil computar a expansão $\alpha$ de um elemento arbitrário $a+b \alpha \in \mathbb{Z}[\alpha]$. Da prova do Lema 5.2 pode-se derivar o seguinte procedimento simples e eficiente com saídas $k_{j}$ em ordem ascendente de $j$.

Algoritmo 5.1:

[1] enquanto $a \neq 0$ ou $b \neq 0$

[2] fazer se $a$ é par (ver nota contida no colorário)

[3] $\quad$ então $k \leftarrow 0$

[4] $\quad$ senão se $2 a+b \neq 0 \quad$ (casos não crítico e semi-crítico)

[5] então

$[6]$

[7]

[8]

[9]

[10]

$k \leftarrow \operatorname{sinal}(2 a+b) \quad(\mathbb{R}(s)=a+(1 / 2) b)$

se $2 a+b=0 \quad$ (caso crítico (a é impar))

então se $a \equiv 1(\bmod 4)$

então $k \leftarrow-1$

[11]

se $a \equiv 3(\bmod 4)$

[12]

então $k \leftarrow 1$

[13]

$$
x \leftarrow(a-k) / 2 ; a \leftarrow x+b ; b \leftarrow-x
$$

Exemplo 5.1. $s=10$.

\begin{tabular}{|c|rrrr|}
\hline interação & $\mathbf{x}$ & $\mathbf{a}$ & $\mathrm{b}$ & $\mathrm{k}$ \\
\hline 1 & & 10 & 0 & 0 \\
2 & 5 & 5 & -5 & 1 \\
3 & 2 & -3 & -2 & -1 \\
4 & -1 & -3 & 1 & -1 \\
5 & -1 & 0 & 1 & 0 \\
6 & 0 & 1 & 0 & 1 \\
7 & 0 & 0 & 0 & \\
\hline
\end{tabular}

Portanto: $10=\langle 10 \overline{1} \overline{1} 10\rangle_{\alpha}=\alpha^{5}-\alpha^{3}-\alpha^{2}+\alpha$.

Nota: Justificando a linha (12) do algoritmo temos que $s=a+b \alpha=s^{\prime} \alpha+$ $k=(A+B \alpha) \alpha+k$. Como $\alpha^{2}=\alpha-2$, temos que $a+b \alpha=A \alpha+B \alpha-2 B+k$. Portanto, $B=(k-a) / 2$ e $A=b+(a-k) / 2$.

\subsection{Conclusão}

Neste capítulo foi descrita a construção de um algoritmo aplicado a curvas elípticas anômalas definidas sobre o corpo $\mathbb{F}_{2}$ que é cerca de três vezes mais rápido do que o método binário, quando o corpo base é implementado em 
base normal. Essa eficiência não é devida à utilização de pré-computação ou memória adicional. Este algoritmo foi o primeiro a usar as idéias apresentadas no capítulo 4 (o uso do endomorfismo de Frobenius) de maneira eficiente.

Nossa contribuição foi detalhar o artigo [12], correlacionando-o com o capítulo anterior, como pode ser verificado no corpo do Teorema 5.1, Lema 5.1, Lema 5.2, Corolário 5.1 e suas notas, bem como nas observações contidas no referido algoritmo. 


\section{Capítulo 6}

\section{Método de Solinas para multiplicação em curvas definidas sobre $\mathbb{F}_{2}$}

Koblitz [ ] introduziu uma família de curvas que admite uma multiplicação escalar elíptica especialmente rápida. Meier e Staffelbach [1] apresentaram um algoritmo para computar a multiplicação em curvas definidas sobre o corpo $\mathbb{F}_{2}$ que é 3 vezes mais rápida do que o método binário.

Neste capítulo é apresentado um algoritmo de Solinas [2], 2 ] para curvas definidas sobre o corpo $\mathbb{F}_{2}$ que é baseado em um novo tipo de representação de inteiros, análogo as expansões na forma não-adjacente (NAF) (ver Seção 3.7.2). Este algoritmo é no mínimo $50 \%$ mais rápido do que o algoritmo de Meier e Staffelbach.

Utilizamos neste capítulo a notação $\tau$ que representa o endomorfismo de Frobenius $\varphi$ em curvas definidas sobre o corpo $\mathbb{F}_{2}$.

\subsection{Multiplicação escalar elíptica}

As curvas binária anômalas (ou ABC's) são extremamente convenientes para. aplicações criptográficas. Estas são as curvas $E^{0}$ e $E^{1}$ definidas sobre $\mathbb{F}_{2}$ por

$$
E^{a}: y^{2}+x y=x^{3}+a x^{2}+1
$$

Seja o escalar $K \in \mathbb{Z}$ e um ponto $P$ de ordem prima $\eta \leq \mathcal{N}_{n}$. A técnica básica para multiplicação escalar elíptica é o algoritmo $N A F$-Binário descrito no Capítulo 3, que utiliza a vantagem da expansão na forma não-adjacente de $K$ na computação de $K P$, onde $P$. Por exemplo:

$$
N A F(29)=(100 \overline{1} 01)
$$

uma vez que $29=32-4+1$. 
Apresentamos novamente o a algoritmo que computa a expansão NAF de um inteiro $K$.

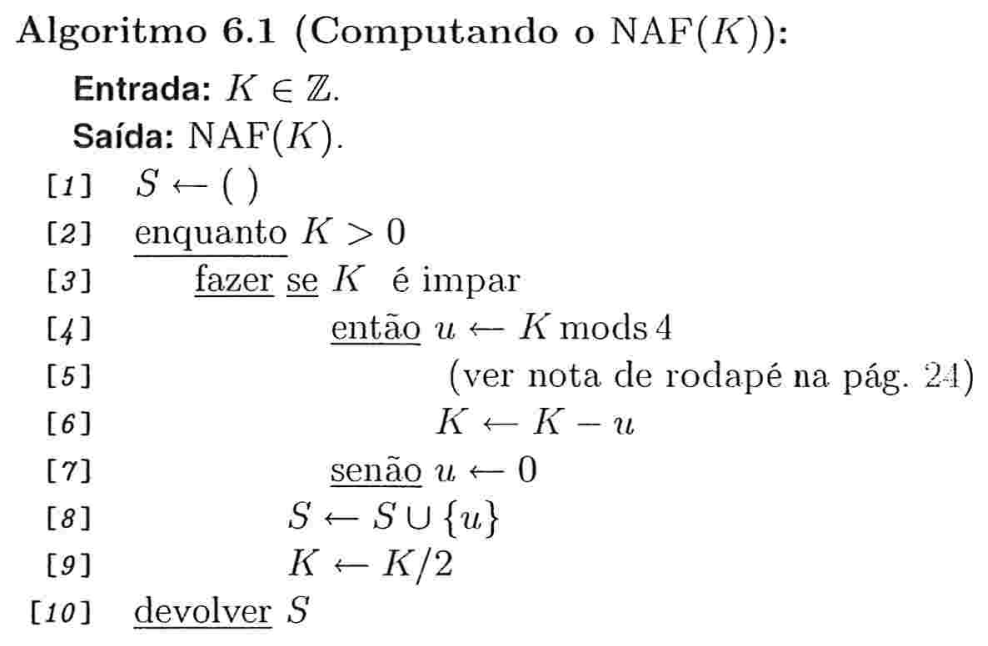

Neste algoritmo, a linha (4) decorre do fato que $K$ é divisível por 4 se somente se $K \equiv 0 \bmod 4$. Assim sendo, o $k_{i}$ obtido nesta linha torna o valor de $K$ da linha (6) divisível por 4. Portanto na próxima iteração $K$ será par.

A Tabela 6.1 ao final do capítulo (página 56) exemplifica uma aplicação do Algoritmo 6.I para derivar a equação 6.1.

Embora o algoritmo tenha sido expresso em termos da aritmética de inteiros, ele pode ser implementado em termos de operações de bits na expansão binária de $K$. Nenhuma operação aritmética é necessária além da adição inteira por 1 .

Apresentamos também, novamente, o algoritmo para multiplicação escalar usando $\operatorname{NAF}(K)$.

Seja a expansão $\operatorname{NAF}(K)=\sum_{i=0}^{l-1} k_{i} 2^{i}$, com $K \in \mathbb{Z}$, a multiplicação escalar elíptica é computada como se segue:

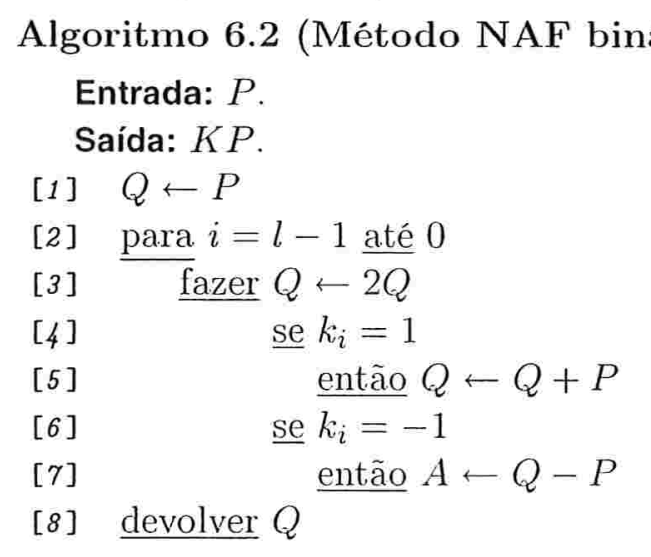


O comprimento de uma expansão NAF de um inteiro $K$ possui comprimento $\leq l+1$, onde $l$ é o comprimento da expansão binária de $K$ e $l \leq m=\log _{2}\left(\mathcal{N}_{n}\right) \approx \log _{2}\left(2^{n}\right)=n$. A densidade média de coeficientes não-zero nesta expansão é $1 / 3$ [ī]. Segue que o custo do Algoritmo 6.2 é de $\sim m$ duplicações e $\sim m / 3$ adições.

\subsection{Expansão NAF $\tau$-ádica}

Denota-se $E_{n}^{a}$ o grupo de pontos $\mathbb{F}_{2^{n}}$ sobre $E^{a}$. Este é o grupo em que os protocolos de chave pública serão executados. O grupo deve ser escolhido tal que o problema do logaritmo discreto de seus elementos seja computacionalmente difícil de ser computado . Portanto, $\mathcal{N}_{n}^{a}$ deveria ser divisível por um número primo grande [1]. Idealmente $\mathcal{N}_{n}^{a}$ deveria ser um primo ou o produto de um primo e um pequeno inteiro, o cofator ( ver seção 4.4.1).

O melhor resultado esperado é que $\mathcal{N}_{n}^{1}$ seja duas vezes um número primo. Isto acontece com uma freqüência relativa no caso de $E^{1}$. Os valores de $n<512$ em que $\mathcal{N}_{n}^{1}$ é duas vez um número primo são:

$$
n=3,5,7,17,19,23,101,109,113,163,283,311,331,347,359
$$

O melhor resultado esperado entre as curvas $E^{0}$ é aquele em que $\mathcal{N}_{n}^{0}$ é 4 vezes um primo. Os valores de $n<512 \mathrm{em}$ que isto acontece são:

$$
n=5,7,13,19,23,41,83,97,103,107,233,277,283,349,409
$$

Desde que as $\mathrm{ABC}$ 's são definidas sobre $\mathbb{F}_{2}$, elas tem a propriedade de que se $P=(x, y)$ é um ponto em $E^{a}$, então o ponto $\left(x^{2}, y^{2}\right)$ também é um ponto sobre $E^{a}$, devido ao endomorfismo de Frobenius para a curva $E^{a}: \tau(x, y)=\left(x^{2}, y^{2}\right)$.

Seja $\epsilon=(-1)^{1-a}$. O endomorfismo de Frobenius para $E^{a}$ obedece a equação característica $\tau^{2}-\epsilon \tau+2=0$ (portanto $\left(x^{4}, y^{4}\right)-\epsilon\left(x^{2}, y^{2}\right)+2(x, y)=$ 0 para todo $\left.(x, y) \in E_{n}^{a}\right)$.

Isto significa que o endomorfismo de Frobenius $\tau(x, y)=\left(x^{2}, y^{2}\right)$ pode ser considerado como uma multiplicação por um número complexo $\tau$ satisfazendo $a$

$$
\tau^{2}-\epsilon \tau+2=0 .
$$

Explicitamente este número é $\tau=(\epsilon+\sqrt{-7}) / 2$.

Combinando o mapeamento de Frobenius $\tau(x, y)=\left(x^{2}, y^{2}\right)$ com a multiplicação ordinária, pode-se multiplicar pontos em $E_{n}^{a}$ por qualquer elemento do do anel $\mathbb{Z}[\tau]$. Dizemos que $E^{a}$ possui multiplicações complexas por $\tau$.

A razão desta propriedade ser útil para multiplicação elíptica é que a multiplicação por $\tau$, sendo implementada por uma elevação ao quadrado, não possui custo computacional apreciável quando o corpo $\mathbb{F}_{2^{n}}$ é representado na base normal (ver apendire A). Portanto na computação de $K P$ é vantajoso 
considerar $K$ como um elemento de $\mathbb{Z}[\tau]$ ao invés de apenas um inteiro. Mais precisamente, substituir a expansão binária com sinal de $K$ pela expansão $\tau$-ádica, isto é, uma representação de $K$ como a soma e diferença de distintas potências de $\tau$.

Por exemplo, com $a=1$ temos:

$$
9=\tau^{5}-\tau^{3}+1
$$

onde $\tau=(1+\sqrt{-7}) / 2$ (basta fazer as contas). Portanto, se $P=(x, y)$ é um ponto de $E^{1}$, então

$$
9 P=\left(x^{32}, y^{32}\right)-\left(x^{8}, y^{8}\right)+(x, y)
$$

No exemplo 6.2 tem-se o que se denomina uma expansão NAF $\tau$-ádica do número 9, desde que não existem 2 termos consecutivos que são diferentes de zero $\left(k_{i} k_{i+1} \neq 0\right)$. Tanto Koblitz ([1]) quanto Meier e Staffelbach ([12]) usam expansões binárias $\tau$-ádica com sinal, mas nenhuma delas tem a propriedade de não-adjacência.

Como veremos, a expansão NAF $\tau$-ádica é uma melhoria em relação a expansão $\tau$-ádica, da mesma forma que o NAF é uma melhoria em relação a expansão binária. A NAF $\tau$-ádica possui propriedades análogas ao NAF de inteiros, sendo que todo elemento do anel $\mathbb{Z}[\tau]$ tem uma única NAF $\tau$-ádica.

Para a obtenção da NAF $\tau$-ádica é necessário identificar que $x+y \tau$ é divisível por $\tau$ se e somente se $x$ é par e que é divisível por $\tau^{2}$ se e somente se $(x-2 y) \equiv 0 \bmod 4$. Esta duas relações são utilizadas na construção do algoritmo que computa a expansão NAF $\tau$-ádica.

A primeira relação vem do fato de que se $x+y \tau$ é divisível por $\tau$, então

$$
x+y \tau=(u+v \tau) \tau=-2 v+(u+\epsilon v) \tau^{1},
$$

ou seja, $x$ deve ser par e do fato de que, se $x=2 v$, então

$$
(x+y \tau) / \tau=(y+(x / 2) \epsilon)-(x / 2) \tau .
$$

A segunda relação vem do fato de que todo $x+y \tau$, múltiplo de $\tau^{2}$, é da forma

$$
x+y \tau=(u+v \tau) \tau^{2}=(u+v \tau)(\epsilon \tau-2)
$$

e isto implica que $x=-2(u+\epsilon v)$ e $y=\epsilon u-v$. Ao computarmos $x-2 y$, obtemos que

$$
(x-2 y) \equiv(-(u+v)(2+2 \epsilon)) \equiv 0 \quad \bmod 4
$$

\footnotetext{
${ }^{1} \mathrm{O}$ Corolário 5.1, na página 41 contém explicação similar
} 
Apresentamos o algoritmo que computa a NAF $\tau$-ádica. Ele é análogo ao algoritmo 6.1 que gera a expansão NAF de um inteiro, com a diferença de que a divisão por 2 foi substituída pela divisão por $\tau$.

$\mathrm{O}$ anel $\mathbb{Z}[\tau]$ é um domínio Euclidiano em relação a função norma (ver Seção 4.5.2)

$$
N(x+y t)=x^{2}+\epsilon x y+2 y^{2}
$$

Desde que $\tau$ possui norma igual a 2 , os possíveis restos da divisão por $\tau$ são 0 e \pm 1 . O resto é escolhido tal que o novo quociente obtido seja divisível por $\tau$ (isto é, tendo a parte real par).

Algoritmo 6.3 (NAF $\tau$-ádica):

Entrada: $x_{0}, y_{0}$

[1] $x \leftarrow x_{0} ; \quad y \leftarrow y_{0}$
[2] $S \leftarrow()$
[3] enquanto $x \neq 0$ ou $y \neq 0$

[4] $\underline{\text { fazer }} \underline{\text { se } x}$ é impar

[5] então $u \leftarrow(x-2 y)$ mods 4

[6] (ver nota de rodapé na pág. 2⿺)

[7] $\quad x \leftarrow x-u$

[8] Senão $u \leftarrow 0$

[9] $\quad S \leftarrow S \cup\{u\}$

[10] $\quad(x, y) \leftarrow(y+\epsilon x / 2,-x / 2)$

[11]

[12] devolver $S$

Note que o valor de $u \in\{ \pm 1\}$ obtido na linha (5) torna $(x-u)+y \tau$ divisível por $\tau^{2}$ e $((x-u)+y \tau) / \tau$ obtido na linha (10) divisível por $\phi$. Asssim sendo, na próxima iteração $x+y \tau$ será divisível por $\tau$ ou, de maneira equivalente, $x$ será par.

Note também que as linhas (4),(5) e (10) do algoritmo 6.3 decorrem, respectivamente, de $x+y \tau$ ser divisível por $\tau$ se e somente se $x$ for par ; $x+y \tau$ ser divisível por $\tau^{2}$ se e somente se $(x-2 y) \equiv 0 \bmod 4$ e ainda da identidade

$$
(x+y \tau) / \tau=(y+(x / 2) \epsilon)-(x / 2) \tau .
$$

quando $x$ for par.

Uma exemplo da aplicação do algoritmo 6.3 foi a derivação da equação 6.2 que foi obtida considerando-se $a=1, x=9$ e $y=0$. Os resultados são mostrados na Tabela 6.2 (página 56).

Um argumento similar ao de [1] para o caso NAF prova que a densidade média de termos diferentes de zero das expansões NAF $\tau$-ádica é de $1 / 3$.

Uma desvantagem é que a NAF $\tau$-ádica de um inteiro $K$ é quase duas vezes maior do que o NAF ordinário. Isto é porque o Algoritmo 6.3 começa com $K$, que é um elemento de $\mathbb{Z}[\tau]$ com norma $K^{2}$, é repetidamente dividido 
por $\tau$ que possui norma 2 e portanto $\log _{2} K^{2}=2 \log _{2} K$, ou seja, duas vezes o comprimento da expansão NAF de $K$ que é $\log _{2} K$.

A solução é adotar a seguinte modificação de Meier e Staffelbach ([12]). Como a multiplicação por $\tau$ é implementada por um deslocamento circular de um bit de cada string de bits de tamanho $n$ que representa as coordenadas do ponto $P=(x, y)$, a multiplicação por $\tau^{n}$ envolve $n$ tais deslocamentos, retornando cada coordenada ao seu estado original. Em outras palavras $\tau^{n} P=P$ para todos os $P \in E_{n}^{a}$. Segue que, se $\alpha$ e $\beta$ são elementos de $\mathbb{Z}[\tau]$ $\operatorname{com} \alpha \equiv \beta\left(\bmod \left(\tau^{n}-1\right)\right)$, então $\alpha P=\beta P$ para todos os $P \in E_{n}^{a}{ }^{2}$.

Portanto, para multiplicar por $K$, não é necessário trabalhar com $K$, mas com o resto obtido da divisão por de $K$ por $\left(\tau^{n}-1\right)$. Desde que $\mathbb{Z}[\tau]$ é um domínio Euclidiano, este resto terá norma menor do que $N\left(\tau^{n}-1\right)$.

Mas $N\left(\tau^{n}-1\right)=\mathcal{N}_{n}^{a}<2^{n}$. Portanto o NAF $\tau$-ádica do resto terá comprimento máximo de $\sim n$, pois $\log _{2} 2^{n}=n$. Além disso, a densidade média é ainda somente $1 / 3$.

Para implementar esta melhoria, é necessário um algoritmo de divisão em $\mathbb{Z}[\tau]$.

\subsection{Divisão e redução modular em $\mathbb{Z}[\tau]$}

Dado um dividendo $\gamma=u+v \tau$, um divisor $\delta=r+s \tau$, deseja-se encontrar um quociente $\kappa=w+z \tau$ e um resto $\rho=x+y \tau$, tal que

$$
\gamma=\kappa \delta+\rho,
$$

onde $\rho$ possui norma mínima. Para se fazer isto, obtêm-se $\kappa$ através de uma operação de arredondamento de $\gamma / \delta$ encontrando posteriormente $\rho$. Isto é, faz-se

$$
\lambda=\frac{\gamma}{\delta}=\frac{\gamma \bar{\delta}}{\delta \bar{\delta}}=\frac{\gamma \bar{\delta}}{N(\delta)}=\frac{g_{0}+g_{1} \tau}{N} .
$$

Encontra-se $\kappa$ fazendo

$$
\kappa=\text { Arredondamento }\left(\frac{g_{0}}{N}+\frac{g_{1}}{N} \tau\right),
$$

e se obtém $\rho$ via

$$
\rho=\gamma-\kappa \delta .
$$

Note que

$$
\delta \bar{\delta}=\left(r+\frac{\epsilon s}{2}+\frac{\sqrt{7}}{2} s i\right)\left(r+\frac{\epsilon s}{2}-\frac{\sqrt{7}}{2} s i\right)=r^{2}+\epsilon r s+2 s^{2}=N(\delta)
$$

O Algoritmo 6.4 tem como entrada o dividendo $\gamma=u+v \tau$, o divisor $\delta=r+s \tau$ e como saída um quociente $\kappa=w+z \tau$ e um resto $\rho=x+y \tau$.

\footnotetext{
${ }^{2}$ ver explicação similar na parte final da demonstração do Teorema . ..1 na página 43
} 
Algoritmo 6.4 (Divisão no anel $\mathbb{Z}[\tau]$ ):

Entrada: $u, v, r, s$.

$$
\begin{array}{ll}
\text { [1] } & g_{0} \leftarrow u r+\epsilon u s+2 v s \\
{[2]} & g_{1} \leftarrow v r-u s \\
{[3]} & N \leftarrow r^{2}+\epsilon r s+2 s^{2} \\
{[4]} & \lambda_{0} \leftarrow g_{0} / N \\
{[5]} & \lambda_{1} \leftarrow g_{1} / N \\
{[6]} & (w, z) \leftarrow \text { Arredondamento }\left(\lambda_{0}, \lambda_{1}\right) \\
{[7]} & x \leftarrow u-r w+2 s v \\
{[8]} & y \leftarrow v-s w-r z-\epsilon s z \\
{[9]} & \text { devolver } w, z, x, y
\end{array}
$$

Note que

$$
\begin{gathered}
g_{0}+g_{1} \tau=\gamma \bar{\delta}= \\
=\left(u+\frac{\epsilon v}{2}+\frac{\sqrt{7}}{2} v i\right)\left(r+\frac{\epsilon s}{2}-\frac{\sqrt{7}}{2} s i\right)=(u r+\epsilon u s+2 v s)+(v r-u s) \tau
\end{gathered}
$$

Note também que as linhas (7) e (8) surgem de

$$
\begin{aligned}
u+v \tau & =(w+z \tau)(r+s \tau)+(x+y \tau) \\
& =w r+w s \tau+r z \tau+z s \tau^{2}+x+y \tau \\
& =w r+w s \tau+r z \tau+z s(-1)^{1-a} \tau-2 z s+x+y \tau \\
& =(w r-2 z s+x)+(y+w s+r z+z s \epsilon) \tau
\end{aligned}
$$

Portanto,

$$
\begin{aligned}
& x \leftarrow u-r w+2 s z \\
& y \leftarrow v-s w-r z-\epsilon s z .
\end{aligned}
$$

O Algoritmo 6.4 faz um arredondamento dos valores de $\left(\lambda_{0}, \lambda_{1}\right)$ em $\mathbb{Z}[\tau]$. A lógica do algoritmo é descrita por Solinas em [24]. Neste algoritmo $\operatorname{Round}(x)=\lfloor x+1 / 2\rfloor$, onde $x \in \mathbb{R}$. 
Algoritmo 6.5 (Arredondamento em $\mathbb{Z}[\tau]$ ):

Entrada: números reais $\lambda_{0}, \lambda_{1}$ especificando o número complexo $\lambda=$ $\lambda_{0}+\lambda_{1} \tau$.

Saída: números reais $w, z$ especificando $w+z \tau=\operatorname{Arredondamento}(\lambda)$.

[1] $f_{0} \leftarrow \operatorname{Round}\left(\lambda_{0}\right)$

[2] $f_{1} \leftarrow \operatorname{Round}\left(\lambda_{1}\right)$

[3] $d_{0} \leftarrow \lambda_{0}-f_{0}$

[4] $d_{1} \leftarrow \lambda_{1}-f_{1}$

[5] $h_{0} \leftarrow 0$

[6] $h_{1} \leftarrow 0$

[7] $d \leftarrow 2 d_{0}+\epsilon d_{1}$

[8] se $d \geq 1$

[9] então se $d_{0}-3 \epsilon d_{1}<-1$

[10] $\quad$ então $h_{1} \leftarrow \mu$

[11] $\quad \underline{\text { senão }} h_{0} \leftarrow 1$

[12] Senão se $d_{0}+4 \epsilon d_{1} \geq 2$

[13] $\quad$ então $h_{1} \leftarrow \mu$

[14] se $n<-1$

[15] $\quad$ então se $d_{0}-3 \epsilon d_{1} \geq 1$

[16] então $h_{1} \leftarrow-\mu$

[17] Senão $h_{0} \leftarrow-1$

[18] Senão se $d_{0}+4 \epsilon d_{1}<-2$

[19] $\quad$ então $h_{1} \leftarrow-\mu$

[20] $w \leftarrow f_{0}+h_{0}$

[21] $z \leftarrow f_{1}+h_{1}$

[22] devolver $q_{0}, q_{1}$

Para aplicar o Algoritmo 6.4 é necessário expressar $\left(\tau^{n}-1\right)$ na forma $r+s \tau$. Isto é feito via a seqüência de Lucas:

$$
\begin{aligned}
& U_{0}=0 \\
& U_{1}=1 \\
& U_{i}=\epsilon U_{i-1}-2 U_{i-2} .
\end{aligned}
$$

Para $i \geq 2$ é fácil provar por indução em $n$ que $\tau^{n}=U_{n} \tau-2 U_{n-1}$ e portanto $\tau^{n}-1=U_{n} \tau-\left(2 U_{n-1}+1\right)$

\subsection{Algoritmo de multiplicação}

O algoritmo 6.6 computa $K P$ em $E_{n}^{a}$. No passo (a), efetua-se a redução de $K$ obtendo-se $K^{\prime}$ (seção 6.3). No passo (b) computa-se a expansão NAF $\tau$-ádica de $K^{\prime}$ (seção 6.2). As linhas (1-9) efetuam a multiplicação escalar elíptica a partir da expansão NAF $\tau$-ádica de $K^{\prime}$. 
Algoritmo 6.6 (Multiplicação escalar em ABC's):

(a) Divida $K$ por $U_{n} \tau-\left(2 U_{n-1}+1\right)$ (algoritmo 6.4) obtendo $K^{\prime}$.

(b) Compute NAF $\tau$-ádica de $K^{\prime}$ obtendo $\left\langle e_{l}, e_{l-1}, \ldots, e_{1}, e_{0}\right\rangle$.

[1] $Q \leftarrow e_{l} P$

[2] para $i=l-1$ até 0

[3] fazer $Q \leftarrow \tau(Q)$

[4] $\quad \underline{\text { se }} e_{i}=1$

[5] $\quad$ então $Q \leftarrow Q+P$

[6] $\quad$ se $e_{i}=-1$

[7] $\quad$ então $Q \leftarrow Q-P$

[8]

[9] devolver $Q$

Desde que $l \leq m \approx n$, então o Algoritmo 6.6 requer $m / 3$ adições e nenhuma duplicação. É no mínimo $50 \%$ mais rápido do que o algoritmo de Meier-Staffelbach [12] apresentado no capítulo anterior.

Apresentamos abaixo um exemplo do funcionamento do algoritmo 6.6 para a curva $E_{n}^{1}$ considerando $K^{\prime}=9$.

\begin{tabular}{|c|c|c|}
\hline \multicolumn{1}{|c|}{5} & 0 & 4 \\
\hline 1 & $\varphi P$ & -1 \\
\hline$P$ & 2 & $\varphi^{2}(P)-P$ \\
\hline 3 & 0 & 1 \\
\hline 0 & $\varphi^{4}(P)-\varphi^{2}(P)$ & $\varphi^{5}(P)-\varphi^{3}(P)+P$ \\
\hline
\end{tabular}




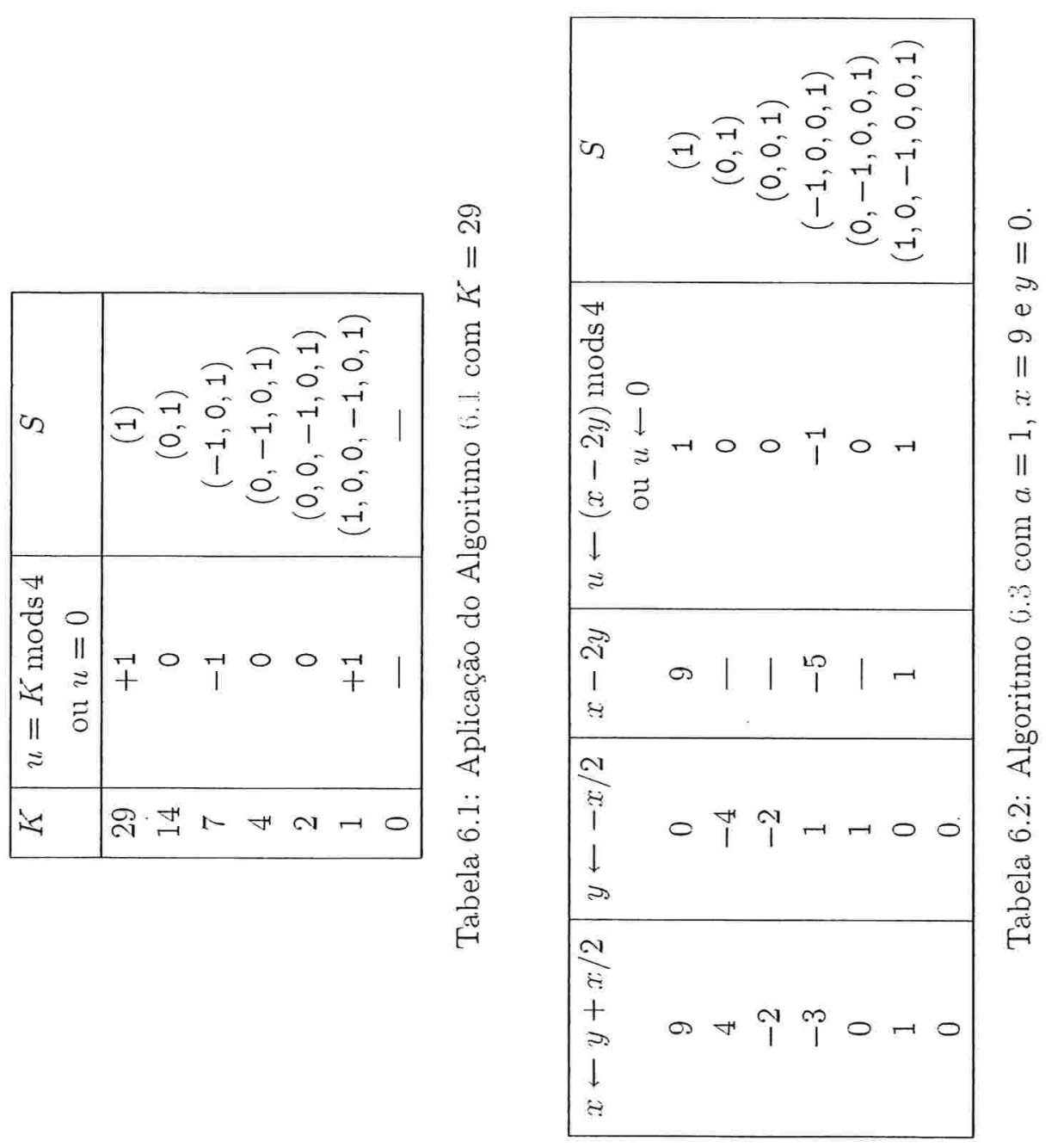




\subsection{Conclusão}

Neste capítulo foi descrito a construção de um algoritmo aplicado a curvas binárias anômalas que propõe usar a propriedade da não-adjacência para melhorar o custo computacional.

Nossa contribuição foi consolidar as informações contidas nos artigos [21, 24, correlacionando-os com os capítulos anteriores, bem como detalhar a construção do algoritmo, como pode ser observado nas notas que inserimos ao longo do capítulo.Explicamos também de maneira mais clara a obtenção do algoritmo que computa a divisão no anel $\mathbb{Z}[\tau]$ (Seção 6.3). 


\section{Capítulo 7}

\section{Método de Volker Müller}

Este capítulo discute um algoritmo para multiplicação de pontos em curvas elípticas definidas sobre pequenos corpos de característica 2 que é no mínimo duas vezes mais rápido do que o algoritmo binário.

Meier e Staffelbach [1] mostraram como aumentar a velocidade da multiplicação de pontos na curva anômala $y^{2}+y x=x^{3}+1$ definida sobre $\mathbb{F}_{2}$. Este capítulo descreve as idéias apresentadas por Volker Müller [ para acelerar a multiplicação em uma curva elíptica não supersingular definida sobre pequenos corpos de característica 2 , ou seja, $\mathbb{F}_{q}$, onde $q=4, q=8 \mathrm{e}$ $q=16$ (equação 7.1 ).

No método descrito neste capítulo exige-se $q / 2$ pré-computações.

Seja $\mathbb{F}_{q}$ um corpo finito com $q$ elementos, onde $q$ é uma pequena potência de 2. Uma curva elíptica (não supersingular) $E$ sobre $\mathbb{F}_{q}$ é definida pela. equação

$$
y^{2}+x y=x^{3}+a x^{2}+b,
$$

onde $a, b \in \mathbb{F}_{q}$ e $b \neq 0$. O conjunto de pontos $E_{n}$ sobre o corpo estendido $\mathbb{F}_{q^{n}}$ é dado pelo conjunto de soluções em $\mathbb{F}_{q^{n}}^{2}$ de ( $(\bar{r} .1)$ junto com o ponto no infinito denotado por $\mathcal{O}$.

O endomorfismo de Frobenius de $E$ é formulado como

$$
\begin{aligned}
\varphi: & E\left(\mathbb{F}_{q}\right) \rightarrow E\left(\mathbb{F}_{q}\right) \\
& (x, y) \mapsto\left(x^{q}, y^{q}\right) .
\end{aligned}
$$

Este endomorfismo satisfaz a equação

$$
\alpha^{2}-c \alpha+q=0
$$

onde $c \in \mathbb{Z}$ e $|c| \leq 2 \sqrt{q}$. Desde que $E$ é não supersingular, o traço $c$ deve ser ímpar, uma vez que a característica do corpo é 2 . 


\subsection{Representando inteiros como uma soma de potên- cias de $\varphi$}

O Lema 7.1 prova a existência de uma "divisão por $\alpha$ com resto" no anel $\mathbb{Z}[\alpha]$. O Teorema 7.1 prova que existe uma expansão definida conforme o Lema 7.1, bem como fornece o comprimento desta expansão.

Lema 7.1. Seja $s \in \mathbb{Z}[\alpha]$. Existe um inteiro $r \in \mathbb{Z},-q / 2 \leq r \leq q / 2$, e um elemento $t \in \mathbb{Z}[\alpha]$, tal que $s=t \alpha+r$. Se escolhermos $r \in\{-q / 2+1, q / 2\}$, então $r$ e t são únicos.

Demonstração. Seja $s=s_{2} \alpha+s_{1} \operatorname{com} s_{1}, s_{2} \in \mathbb{Z}$. Deseja-se encontrar inteiros $t_{1}, t_{2}, r \in \mathbb{Z}$, tal que

$$
s_{2} \alpha+s_{1}=\left(t_{2} \alpha+t_{1}\right) \alpha+r
$$

Usando o polinômio mínimo de $\alpha$, mostrado na equação (7.3), o lado direito da equação (7.4) é transformado em:

$$
\begin{aligned}
\left(t_{2} \alpha+t_{1}\right) \alpha+r & =t_{1} \alpha+t_{2} \alpha^{2}+r \\
& =t_{1} \alpha+t_{2}(c \alpha-q)+r \\
& =\left(t_{1}+c t_{2}\right) \alpha+\left(-t_{2} q+r\right) .
\end{aligned}
$$

Portanto:

$$
s_{2} \alpha+\dot{s}_{1}=\left(t_{1}+t_{2} c\right) \alpha+\left(-t_{2} q+r\right)
$$

e conclui-se que $s_{1}=-t_{2} q+r$. Como esta equação deve ser resolvida dentro dos números inteiros, temos que $r \equiv s_{1}(\bmod q)$.

Escolhendo $r$ como menor resíduo absoluto de $s_{1}(\bmod q)$, computa-se

$$
t_{2}=\left(r-s_{1}\right) / q \quad \text { e } \quad t_{1}=s_{2}-c t_{2}=s_{2}-c\left(r-s_{1}\right) / q
$$

A unicidade de $r$ e $t$ segue do fato que $\{-q / 2+1, q / 2\}$ forma um conjunto completo mínimo de resíduos módulo $q$.

Pode-se fazer uma iteração no processo de divisão por $\alpha$ com resto. Este procedimento conduz à expansão de Frobenius de um elemento $s \in \mathbb{Z}[\alpha]$.

Seja $\|s\|$ o tamanho Euclidiano de $s$. O próximo teorema fornece o comprimento da expansão de Frobenius de $s$.

Teorema 7.1. Seja $q \geq 4$ e $s \in \mathbb{Z}[\alpha]$. Se $l=\left\lceil 2 \log _{q}\|s\|\right\rceil+3$, ent.ão existe inteiros $r_{j} \in\{-q / 2, \ldots, q / 2\}, 0 \leq j \leq l$, tais que $s=\sum_{j=0}^{l} r_{j} \varphi^{j}$.

Demonstração - primeira parte. Faça $s_{0}=s$ e defina para $j \geq 0$ indutivamente os elementos $s_{j+1} \in \mathbb{Z}[\alpha]$ por

$$
s_{j}=s_{j+1} \alpha+r_{j}
$$


onde $r_{j} \in\{-q / 2, \ldots, q / 2\}$.

Pelo Lema 7.1, tais inteiros $r_{j}$ sempre existem. Os elementos $s_{j}, 0 \leq j \leq$ $i+1$, conduzem a uma expansão da forma

$$
\begin{aligned}
s_{0}= & s_{1} \alpha+r_{0} \\
= & \left(s_{2} \alpha+r_{1}\right) \alpha+r_{0} \\
= & \left(\left(s_{3} \alpha+r_{2}\right) \alpha+r_{1}\right) \alpha+r_{0} \\
& \vdots \\
= & \sum_{j=0}^{i} r_{j} \alpha^{j}+s_{i+1} \alpha^{i+1}
\end{aligned}
$$

Usando a desigualdade triangular na equação (7.5) e o fato de que $\left\|r_{i}\right\| \leq$ $q / 2$, então

$$
\left\|s_{i+1}\right\| \leq \frac{\left\|s_{i}\right\|+\left\|r_{i}\right\|}{\sqrt{q}} \leq \frac{\left\|s_{i}\right\|+q / 2}{\sqrt{q}} .
$$

Pode-se mostrar por indução que

$$
\left\|s_{i+1}\right\|<\frac{\left\|s_{0}\right\|}{q^{(i+1) / 2}}+\frac{1}{2} \sum_{u=-1}^{i-1} q^{-u / 2} .
$$

Para $q \geq 4$, essa expressão pode ser limitada a

$$
\left\|s_{i+1}\right\|<\frac{\left\|s_{0}\right\|}{q^{(i+1) / 2}}+\frac{1}{2}\left(\sqrt{q}+\frac{\sqrt{q}}{\sqrt{q}-1}\right) \leq \frac{\left\|s_{0}\right\|}{q^{(i+1) / 2}}+\sqrt{q} \quad 1
$$

$$
\begin{gathered}
\left\|s_{i+1}\right\|<\frac{\left\|s_{0}\right\|}{q^{(i+1) / 2}}+\frac{1}{2}\left(\sqrt{q}+1+\frac{1}{\sqrt{q}}+\frac{1}{q}+\frac{1}{q \sqrt{q}}\right. \\
\left.+\frac{1}{q^{2}}+\frac{1}{q^{2} \sqrt{q}}+\frac{1}{q^{3}}+\frac{1}{q^{3} \sqrt{q}}+\ldots\right) \\
\left\|s_{i+1}\right\|<\frac{\left\|s_{0}\right\|}{q^{(i+1) / 2}}+\frac{1}{2}\left(\sqrt{q}+\frac{1}{\sqrt{q}}\left(1+\frac{1}{q}+\frac{1}{q^{2}}+\frac{1}{q^{3}} \ldots\right)\right. \\
\left.+1+\frac{1}{q}+\frac{1}{q^{2}}+\frac{1}{q^{3}}+\ldots\right) \\
\left\|s_{i+1}\right\|<\frac{\left\|s_{0}\right\|}{q^{(i+1) / 2}}+\frac{1}{2}\left(\sqrt{q}+\left(\frac{\sqrt{q}+1}{\sqrt{q}}\right)\left(1+\frac{1}{q}+\frac{1}{q^{2}}+\frac{1}{q^{3}} \ldots\right)\right) \\
\cong \frac{\left\|s_{0}\right\|}{q^{(i+1) / 2}}+\frac{1}{2}\left(\sqrt{q}+\frac{\sqrt{q}}{\sqrt{q}-1}\right)
\end{gathered}
$$


Portanto, para $i \geq 2 \log _{q}\left\|s_{0}\right\|-1,\left\|s_{i+1}\right\|<\sqrt{q}+1 .^{2}$

Demonstração - segunda parte. Restrinja o comprimento da expansão para elementos de tamanho Euclidiano limitado por $\sqrt{q}+1$, ou seja, pare a computação quando $\left\|s_{i+1}\right\| \geq \sqrt{q}+1$.

Lembre que a norma de elemento arbitrário $a+b \alpha \in \mathbb{Z}[\alpha]$ é $N(a+b \alpha)=$ $\|a+b \alpha\|^{2}$ (ver Seção 4.5.2) e que

$$
N(a+b \alpha)=a^{2}+c a b+q b^{2}=(a+c b / 2)^{2}+(1 / 4)\left(4 q-c^{2}\right) b^{2}
$$

Portanto deve-se que examinar o comprimento de expansões para todos os elementos em $\mathbb{Z}[\alpha]$ de norma menor do que $(\sqrt{q}+1)^{2}$.

Assuma que $a+b \alpha$ seja um desses elementos, ou seja, $N(a+b \alpha)<$ $(\sqrt{q}+1)^{2}$. Desde que $c$ é ímpar, verifica-se na equação (7.6) que $|b| \leq 1^{3}$.

No caso em que $b=0$ temos que $|a|<\sqrt{q}+1$. Desde que $\sqrt{q}+1<q / 2$ para todos os $q \geq 8$, conclui-se que temos $|a| \leq q / 2$ para todos os $q$, e o comprimento da expansão é $1^{4}$.

No caso em que $|b|=1$, o limite superior $|a|<|c| / 2+\sqrt{q}+1 \leq 2 \sqrt{q}+1 \leq$ $(3 / 2) q$ é obtido. Se $|a|<q / 2$, então obviamente $a \pm \alpha$ é por si mesma uma expansão de Frobenius válida de comprimento 2. Se $|a|>q / 2$ usa-se o polinômio mínimo da equação (7.3) de $\varphi\left(\alpha^{2}=c \alpha-q\right)$ e se obtém

$$
a \pm \alpha=(a-q)+(c \pm 1) \alpha-\alpha^{2}=(a-q)+((c \pm 1)-\alpha) \alpha \quad 5
$$

Agora $(a-q)$ esta no intervalo correto $(|a| \leq(3 / 2) q$ e portanto $|a-q| \leq$ $q / 2)$, no entanto, $|c \pm 1|$ poderia ser maior do que $q / 2$. Perceba que há somente duas situações, abaixo discriminadas, em que isso pode acontecer: $q=4, c= \pm 3$ ou $q=8, c= \pm 5^{6}$.

Em todas as outras situações a expansão para $(a+\alpha)$ satisfaz todas as condições e o comprimento da expansão é exatamente 3 .

\footnotetext{
${ }^{2}$ Fazendo $\frac{\left\|s_{0}\right\|}{q^{(i+1) / 2}}<1$, então $(i+1) / 2 \geq \log _{q}\left\|s_{0}\right\|$ e portanto $i \geq 2 \log _{q}\left\|s_{0}\right\|-1$.

${ }^{3} N(a+b \alpha)=a^{2}+c a b+q b^{2} \leq 1+2 \sqrt{q}+q$

${ }^{4} \mathrm{O}$ caso $q=4$ só pode ocorrer quando $|a|<q / 2$

${ }^{5}$ Como $\alpha^{2}=c \alpha-q$, temos que $(a-q)+(c \pm 1) \alpha-\alpha^{2}=a-q+c \alpha \pm \alpha-c \alpha+q=a \pm \alpha$

${ }^{6}$ Como $|c|<2 \sqrt{q},|c|>q / 2$, e $c$ deve ser ímpar, esta restrição só é atendida por $q=4$ e $q=8$, senão vejamos:

$$
\begin{aligned}
& q=4 \Rightarrow 2<|c| \leq 4 \Rightarrow|c|=3 \\
& q=8 \Rightarrow 4<|c| \leq 5,66 \Rightarrow|c|=5 \\
& q=16 \Rightarrow 8<|c| \leq 8
\end{aligned}
$$
}


Para as duas situações especiais obtemos as seguintes expansões:

$$
\begin{array}{ll}
a \pm \alpha=(a-4)+2 \alpha^{2}-\alpha^{3} & \text { (e os casos simétricos) } \\
a \pm \alpha=(a-8)+2 \alpha+4 \alpha^{2}-\alpha^{3} & \text { (e os casos simétricos) }
\end{array}
$$

isto é, o comprimento dessas expansões é menor ou igual a 4

Portanto prova-se que todos os elementos de $\mathbb{Z}[\alpha]$ de tamanho Euclidiano menor que $\sqrt{q}+1$ possui uma expansão de Frobenius de comprimento de no máximo 4.

Conclusão:

Como se sabe que para $i \geq 2 \log _{q}\left\|s_{0}\right\|-1$ tem-se que $\left\|s_{i+1}\right\|<\sqrt{q}+1 \leq$ $q / 2$, então para $l \geq 2 \log _{q}\left\|s_{0}\right\|+3$ tem-se que $s_{i+1}=0$ e o teorema está provado.

O Teorema 7.1. afirma a existência de uma expansão de Frobenius de um dado comprimento, no entanto não está claro se é possível computar esta expansão em um tempo razoável. Um algoritmo para computar tal expansão baseia-se na divisão iterativa com um resto dado pela equação (7.5). Infelizmente, existem duas possibilidades para o resto $r_{j}$ se $r_{j} \equiv(q / 2)(\bmod q)$.

Relembrando a prova do Teorema 7.1 , conclui-se que não existe problema em escolher este ou aquele candidato, desde que o índice da iteração seja menor do que $2 \log _{q}\left\|s_{0}\right\|-1$. A expansão mais curta para o resto de $a+b \alpha$ com $|a| \leq(3 / 2) q$ e $|b| \leq 1$ pode ser pré-computada e armazenada, tal que é possível computar a expansão de Frobenius cujo comprimento seja limitado pelos valores dados no Teorema 7.].

\subsection{Multiplicação de pontos usando a expansão de Frobenius}

Seja $E$ uma curva elíptica definida sobre um corpo $\mathbb{F}_{q}$, onde $q$ é uma pequena potência de 2. Vamos supor que se deseja computar $K P$ para algum inteiro $K \in \mathbb{N}$ e um ponto $P \in E_{n}$ (consideramos $K$ reduzido a módulo $\mathcal{N}_{n}$ ). Seja $\varphi$ o endomorfismo de Frobenius de $E$. No Teorema 7.1 foi mostrado que é possível expandir o inteiro $K \in \mathbb{N}$ como uma soma de potência do endomorfismo de Frobenius $\varphi$ :

$$
K=k_{j} \sum_{j=0}^{l} \varphi^{j},
$$

onde os coeficientes satisfazem a condição $k_{j} \in\{-q / 2, \ldots, q / 2\}$.

A observação mais importante neste contexto é o fato de que $\varphi(P)$ pode ser computado $\operatorname{com} 2 \log q$ elevações ao quadrado (veja a equação $(7.2)$ (página 58), que é, para pequenos valores $q$, usualmente mais rápido do que a duplicação de um ponto.

O algoritmo consiste das três diferentes partes: 
1. Compute uma pequena tabela de múltiplos de $P$.

2. Compute e armazene uma expansão de Frobenius de $K$, isto é, compute e armazene os coeficientes $k_{0}, \ldots, k_{k}$ em ordem ascendente (direita para a esquerda).

$$
K=\left(\overleftarrow{k_{k}, \ldots, k_{0}}\right)
$$

3. Use a tabela e os coeficientes armazenados desta expansão em ordem descendente para computar $K P$ (esquerda para a direita)

$$
K=\left(\overrightarrow{k_{k}, \ldots, k_{0}}\right)
$$

\section{Algoritmo 7.1 (Multiplicação em curva definida em $\mathbb{F}_{q}$ ):}

Entrada: $K \in \mathbb{N}$ e um ponto $P \in E_{n}$.

Saída: $K P$.

[1] [TABELA PRÉ-COMPUTADA DE PONTOS]

[2] Compute e armazene $i P$ para todos os $1 \leq i \leq q / 2$.

[3] [COMPUTANDO A EXPANSÃO $K=\left(\overleftarrow{k_{k}, \ldots, k_{0}}\right)$ ]

[4] $s_{1} \leftarrow K ; s_{2} \leftarrow 0 ; i \leftarrow 0$

[5] enquanto $\left|s_{1}\right|>q / 2$ ou $\left|s_{2}\right|>1$

[6] fazer $k_{i} \leftarrow s_{1} \operatorname{mods} q$ (compute e armazene)

[7] (ver nota de rodapé na pág. 24)

[8] $\quad h \leftarrow\left(k_{i}-s_{1}\right) / q ; i \leftarrow i+1 ; s_{1} \leftarrow s_{2}-c h$ e $s_{2} \leftarrow h$

[9] [MultiplicaÇÃO $K=\left(\overrightarrow{k_{k}, \ldots, k_{0}}\right)$ ]

[10] $H \leftarrow s_{2} \varphi(P)+s_{1} P$

[11] para $j=i-1$ até 0

[12] $\quad$ fazer se $k_{j} \geq 0$

[13] então $H \leftarrow \varphi(H)+k_{j} P$

[14] $\quad \underline{\operatorname{senão}} H \leftarrow \varphi(H)-\left|k_{j}\right| P$

[15] devolver $H$

A corretude do algoritmo segue do fato de que o loop das linhas (5 a 8) é uma translação direta da sequência introduzida na prova do Teorema 7.1. Então observe que:

$$
\begin{aligned}
K P & =\left(\sum_{j=0}^{l} k_{j} \varphi^{j}\right)(P) \\
& =\varphi\left(\cdots \varphi\left(\varphi\left(k_{l} \varphi(P)+k_{l-1} P\right)+k_{l-2} P\right) \cdots+k_{1} P\right)+k_{0} P
\end{aligned}
$$

O parte crítica no Algoritmo 7.1 é a pré-computação da tabela da linha (2). Todos os inteiros $k_{j}$, nas linhas (13 a 14), e $s_{1}$ na linha (10) são absolutamente limitados por $q / 2$. Portanto não há necessidade de computar os pontos $s_{1} P$ e $\left|k_{j}\right| P$, mas pode-se tomar esses pontos da tabela pré-computada. 
Como já mencionado, existe uma ambiguidade na computação da expansão de Frobenius de $K$. Na linha (6) do Algoritmo 7.1, temos duas escolhas para $k_{i}$ se $k_{i}=(q / 2)(\bmod q)$.

Uma possibilidade para superar este problema é escolher o valor de $k_{i}$ que minimiza a norma do novo elemento $s_{1}+s_{2} \varphi$.

Exemplo 7.1. Seja a curva $E$ definida sobre $\mathbb{F}_{q}$, onde $q=16$ e $c=7$. O endomorfismo de Frobenius $\varphi$ para $E$ satisfaz $\alpha^{2}-7 \alpha+16=0$.

Entrada: $K=100 \in \mathbb{N}$ e um ponto $P \in E_{n}$.

Saída: $K P$.

Pré-computação de pontos:

$P_{1}=P, P_{2}=2 P, P_{3}=3 P, P_{4}=4 P, P_{5}=5 P, P_{6}=6 P, P_{7}=7 P$ e $P_{8}=8 P$.

\begin{tabular}{|c|c|c|c|c|}
\hline \multicolumn{5}{|c|}{ Computação da expansão } \\
\hline \hline $\mathbf{i}$ & $\mathbf{s}_{\mathbf{1}}=\mathbf{s}_{\mathbf{2}}-\mathbf{c} \cdot \mathbf{h}$ & $\mathbf{s}_{\mathbf{2}}=\mathbf{h}$ & $\mathbf{k}_{\mathbf{i}}$ & $\mathbf{h}=\left(\mathbf{k}_{\mathbf{i}}-\mathbf{s}_{\mathbf{1}}\right) / \mathbf{q}$ \\
\hline 0 & 100 & 0 & $100(\bmod 16)=4$ & $(4-100) / 16=-6$ \\
1 & $0-(7)(-6)=42$ & -6 & $42(\bmod 16)=-6$ & $(-6-42) / 16=-3$ \\
2 & $-6-(7)(-3)=15$ & -3 & $15(\bmod 16)=-1$ & $(-1-15) / 16=-1$ \\
3 & $-3-(7)(-1)=4$ & -1 & - & - \\
\hline
\end{tabular}

\begin{tabular}{|c|c|}
\hline \multicolumn{2}{|c|}{ Multiplicação da esquerda para a direita } \\
\hline \hline & $H=4 P-\varphi(P)$ \\
2 & $H=-1 P+\varphi(4 P-\varphi(P))$ \\
1 & $H=-6 P+\varphi(-1 P+\varphi(4 P-\varphi(P)))$ \\
0 & $h=4 P+\varphi(-6 P+\varphi(-1 P+\varphi(4 P-\varphi(P))))$ \\
\hline
\end{tabular}

\subsection{Custo do algoritmo}

Assuma que o multiplicador $K \in \mathbb{N}$ é reduzido a módulo da ordem do grupo da curva usada ${ }^{7}{ }^{8}$.

A parte mais importante no tempo de execução do Algoritmo 7.1 é o número de adições de pontos e o número de execuções do endomorfismo de Frobenius. O número de adições de pontos executados pelo Algoritmo 7.1 com entrada $(K, P)$ é menor ou igual a $(q / 2+m-1)$ e o número de execuções do mapeamento de Frobenius é menor ou igual a $m$, onde $m \leq 2 \log _{q}\|K\|+$ $3=2 \log _{q} \sqrt{q^{n}}+3=n+3$.

\footnotetext{
${ }^{7}$ Ver essa explicação na parte final da demonstração do Teorema 5.1 na página 43

${ }^{8} \mathrm{Na}$ seção 6.3 foi explicado o algoritmo que obtém essa redução
} 


\subsection{Conclusão}

Neste capítulo foi descrito a construção de um algoritmo ([1] ]) aplicado a curvas elípticas definidas sobre pequenos corpos de característica 2 que é no mínimo duas vezes mais rápido do que o método binário. Este algoritmo usa q/2 pré-computações, e sua aplicação não exige que a curva seja anômala.

Nossa contribuição foi detalhar a construção do algoritmo, como pode ser observado nas notas que inserimos ao longo do capítulo, correlacionando-o com os capítulos anteriores. Cabe destacar que, da mesma forma que o algoritmo descrito no capítulo 5, este algoritmo produz uma expansão utilizando o endomorfismo de Frobenius, no entanto não existe aqui a propriedade de não-adjacência (o que ocorre no algoritmo descrito capítulo 6 ). Destaco também que mostramos como utilizar o software MAGMA [1!], para se obter o traço do endomorfismo de Frobenius das curvas $y^{2}+x y=x^{3}+x^{2}+1 \mathrm{e}$ $y^{2}+x y=x^{3}+x^{2}+1$ definidas sobre $\mathbb{F}_{4}, \mathbb{F}_{8}$ e $\mathbb{F}_{16}$ que foram utilizadas na implementação contida no apêndice B). Apresentamos também um exemplo de funcionamento do algoritmo. 


\section{Capítulo 8}

\section{Forma Esparsa Conjunta de Solinas}

O método de Shamir acelera a velocidade da computação de um produto de potências de dois elementos de um grupo multiplicativo $\left(g^{a} h^{b}\right)$, comum em criptografia de chave pública. É baseado na expansão binária e foi projetado para aritmética modular e de corpo finito.

A aritmética em curvas elípticas (um grupo aditivo) utiliza expansões binárias com sinal. Este capítulo mostra a extensão do método de Shamir para curvas elípticas através da especificação de uma representação binária com sinal para um par de inteiros positivos, a Forma Esparsa Conjunta $(\mathrm{FEC})^{1}$ proposta por Solinas [22].

\subsection{Métodos de Shamir}

Seja $G$ um subgrupo de um grupo multiplicativo de elementos diferentes de zero de um corpo finito $\mathbb{F}_{q}$. A operação básica de chave pública em $G$ é a exponenciação: computar $g^{a}$ para um elemento $g \in G$ e um inteiro positivo $a$ através da expansão binária $\bar{e}$ de $a$. Este método requer uma elevação ao quadrado para todo bit em $\bar{e}$ e uma multiplicação por $g$ para todo bit 1 que aparece em $\bar{e}$. Se $l$ é o comprimento de $\bar{e}$, então o método binário custa $\sim l$ elevações ao quadrado e $\sim l / 2$ multiplicações (na média).

É comum a necessidade de computar uma expressão na forma

$$
g^{a} h^{b} .
$$

Grande parte das assinaturas digitais são verificadas através da computação deste tipo de expressão.

\footnotetext{
${ }^{1}$ Joint Sparse Form (JSF) no artigo original
} 


\subsubsection{Método trivial}

O método trivial para calcular a expressão (8.1) é executar duas exponenciações e multiplicar os resultados. Esta abordagem custa $\sim 2 l$ elevações ao quadrado e $\sim l$ multiplicações (na média).

\subsubsection{Método simples de Shamir}

Shamir notou que é desnecessário computar as duas exponenciações separadamente, uma vez que somente o produto é necessário na verificação da assinatura. Portanto, pode-se aumentar a eficiência combinando as duas exponenciações em uma única operação. Abaixo está ilustrado a aplicação desta técnica na computação de $g^{37} h^{20}$.

\begin{tabular}{|c|c|c|c|c|c|c|}
\hline $37=\{$ & 1 & 0 & 0 & 1 & 0 & 1 \\
\hline $20=$ & 0 & 1 & 0 & 1 & 0 & 0 \\
\hline
\end{tabular}

\begin{tabular}{|c|c|c|c|c|c|c|}
\hline $\begin{array}{r}\text { Elevaçāo ao } \\
\text { quadrado }\end{array}$ & 1 & $g^{2}$ & $g^{4} h^{2}$ & $g^{8} h^{4}$ & $g^{18} h^{10}$ & $g^{36} h^{20}$ \\
\hline$g\{$ & $g$ & 一 & - & $g^{9} h^{4}$ & - & $g^{37} h^{20}$ \\
\hline$h\{$ & - & $g^{2} h$ & - & $g^{9} h^{5}$ & & \\
\hline
\end{tabular}

Este método, conhecido como método simples de Shamir custa $\sim l$ elevações ao quadrado e $\sim l$ multiplicações (na média) (número de multiplicações: $l / 2+l / 2=l)$.

\subsubsection{Método rápido de Shamir}

Shamir notou também que se obtém um aumento na velocidade pré-computando e armazenando previamente o produto $g h$. Desta forma, quando se encontra um bit 1 em ambas as expansões binárias, multiplica-se por $g h$ ao invés de se multiplicar por $g$ e $h$ separadamente. Desde que a probabilidade de que 2 bits aleatórios tenham o valor 1 é de $1 / 4$, obtém-se uma economia de $1 / 4$ na multiplicação em relação ao método simples de Shamir (número de multiplicações: $(l / 2)(3 / 4)+(l / 2)(3 / 4)=3 l / 4)$.

A técnica é ilustrada abaixo na computação de $g^{37} h^{20}$. 


\begin{tabular}{|c|c|c|c|c|c|c|}
\hline $37=\{$ & 1 & 0 & 0 & 1 & 0 & 1 \\
\hline $20=\{$ & 0 & 1 & 0 & 1 & 0 & 0 \\
\hline
\end{tabular}

\begin{tabular}{|c|c|c|c|c|c|c|}
\hline $\begin{array}{r}\text { Elevação ao } \\
\text { quadrado }\end{array}$ & 1 & $g^{2}$ & $g^{4} h^{2}$ & $g^{8} h^{4}$ & $g^{18} h^{10}$ & $g^{36} h^{20}$ \\
\hline$g\{$ & $g$ & - & - & - & - & $g^{37} h^{20}$ \\
\hline$h\{$ & - & $g^{2} h$ & - & - & & \\
\hline$g h\{$ & - & - & - & $g^{9} h^{5}$ & & \\
\hline
\end{tabular}

Esta técnica, denominada método rápido de Shamir custa $\sim l$ elevações ao quadrado e $\sim 3 l / 4$ multiplicações (na média). A pré-computação requer uma multiplicação e em seguida o armazenamento do resultado.

\subsection{Expansões binárias com sinal}

Em curvas elípticas ao invés da exponenciação, a operação de interesse é a multiplicação escalar: dado um elemento $P$ e um inteiro $K$, computar $K P$, o resultado da adição de $K$ cópias de $P(P+P+\cdots+P)$.

Uma vez que, encontrar o negativo de $P$ não possui custo computacional significativo, pode-se usar uma expansão binária com sinal (permitindo coeficientes 0 e \pm 1 ), o que é vantajosa se a mesma seja tal que possua peso de Hamming ${ }^{2}$ menor do que o da expansão binária comum para o mesmo número. Um tipo de expansão binária com sinal de um inteiro $K$ é a forma não adjacente, ou o $\operatorname{NAF}(K)$ (ver Seção 3.7.2). A forma não adjacente de um inteiro $K$ é uma expansão binária com sinal com a propriedade de que não existem dois termos consecutivos diferentes de zero e em que a densidade de Hamming é $1 / 3$.

Exemplo 8.1. $N A F(53)=010 \overline{1} 0101$.

A expansão binária com sinal de um inteiro $K$ será denotada por

$$
K=\left\langle u_{m}, \ldots, u_{1}, u_{0}\right\rangle
$$

e isto significa que

$$
K=\sum_{j=0}^{m} u_{j} 2^{j} \quad \text { e } \quad u_{j} \in\{0, \pm 1\} \text { para todos os } j
$$

\footnotetext{
${ }^{2}$ número de termos diferentes de 0
} 
Para se fazer a adaptação do método de Shamir para a configuração adição-subtração, usa-se a expansões binárias com sinal conjuntas, expressões da forma:

$$
\left[\begin{array}{l}
K_{0} \\
K_{1}
\end{array}\right]=\left[\begin{array}{l}
u_{0, m}, \ldots, u_{0,1}, u_{0,0} \\
u_{1, m}, \ldots, u_{1,1}, u_{1,0}
\end{array}\right]
$$

onde cada $u_{i, j} \in\{0, \pm 1\}$.

No primeiro caso, para se adaptar o método de Shamir simples, utiliza-se as duas expansões na forma NAF, como ilustrado no próximo exemplo.

Exemplo 8.2. Método simples de Shamir para computar $11 P+20 Q$

\begin{tabular}{|c|c|c|c|c|c|}
\hline $11=\{$ & 1 & 0 & -1 & 0 & -1 \\
\hline $20=\{$ & 1 & 0 & 1 & 0 & 0 \\
\hline
\end{tabular}

\begin{tabular}{|c|c|c|c|c|c|}
\hline Duplicação \{ & $\mathcal{O}$ & $2 P+2 Q$ & $4 P+4 Q$ & $6 P+10 Q$ & $12 P+20 Q$ \\
\hline$P\{$ & $P$ & - & $3 P+4 Q$ & - & $11 P+20 Q$ \\
\hline$Q\{$ & $P+Q$ & - & $3 P+5 Q$ & - & - \\
\hline
\end{tabular}

Esta abordagem custa $\sim l$ duplicações e $\sim 2 l / 3$ adições na média (adições: $l / 3+l / 3=2 l / 3$ ). Usar o NAF é uma ótima maneira de executar o método simples de Shamir, desde que minimiza o número de termos diferentes de zero em cada expansão binária com sinal.

Fazer a adaptação do método rápido de Shamir para números binários com sinal no entanto não é direto. Uma abordagem trivial é usar novamente o NAF dos dois coeficientes (Joint NAF). Esta técnica requer $\sim l$ duplicações e $5 l / 9$ adições (na média).

Embora eficiente, esta abordagem não é ótima. Em outras palavras, existem outras expansões binárias com sinal conjuntas para pares de inteiros que trabalham melhor do que a Joint NAF, na computação do método rápido de Shammir.

\subsection{Forma Esparsa Conjunta (FEC)}

O número de adições requeridas pelo método rápido de Shamir é o peso de Hamming conjunto de uma expansão binária com sinal conjunta, isto é, o número de colunas diferentes de 0. Para otimizar método rápido de Shamir escolhe-se uma expansão binária com sinal conjunta com peso de Hamming conjunto mínimo (e comprimento mais ou menos mínimo). A Forma Esparsa Conjunta possui as seguintes propriedades:

JS-1 De qualquer três posições consecutivas, no mínimo uma é um zero duplo (uma coluna zero). Em outras palavras, para qualquer $i$ e $j$ positivo, tem-se $u_{i, j+k}=u_{1-i, j+k}=0$ para $k=0, k=1$ ou $k=-1$. 


\begin{tabular}{|c|c|c|}
\hline$u_{i, j+k}=0$ & $u_{i, j}$ & \\
\hline$u_{1-i, j+k}=0$ & & \\
\hline
\end{tabular}

JS-2 Termos adjacentes não possuem sinais opostos. Em outras palavras, nunca ocorre o caso em que $u_{i, j+1} u_{i, j}=-1$.

$$
\begin{aligned}
& \begin{array}{|l|l|l|l|l|}
\hline u_{i, j+1}=0 & u_{i, j}=0, \pm 1 & \\
\hline u_{i, j+1}=0, \pm 1 & u_{i, j}=0 & \\
\hline u_{i, j+1}=+1 & u_{i, j}=+1 & \\
\hline
\end{array} \\
& \begin{array}{|l|l|l|l|}
u_{i, j+1}=-1 & u_{i, j}=-1 & \\
\hline
\end{array}
\end{aligned}
$$

JS-3 Se $u_{i, j+1} u_{i, j} \neq 0$ então $u_{1-i, j+1}= \pm 1$ e $u_{1-i, j}=0$.

$$
\begin{array}{|c|c|}
\hline u_{i, j+1}=+1 & u_{i, j}=+1 \\
\hline u_{1-i, j+1}= \pm 1 & u_{1-i, j}=0 \\
\hline
\end{array} \quad \begin{array}{|c|c|}
\hline u_{i, j+1}=-1 & u_{i, j}=-1 \\
\hline u_{1-i, j+1}= \pm 1 & u_{1-i, j}=0 \\
\hline
\end{array}
$$

Note que o NAF de um inteiro $K_{0}$ é a FEC de $\left\langle K_{0}, K_{1}\right\rangle$, onde $K_{1}=0$ (desde que todas as expansões 0 são expansões binárias com sinal de 0 ).

O próximo exemplo ilustra a vantagem da FEC sobre a Joint NAF. Para

\begin{tabular}{|c|c|c|c|c|c|c|c|c|}
\hline & \multicolumn{8}{|c|}{ Joint NAF } \\
\hline $53=\{$ & 0 & 1 & 0 & -1 & 0 & 1 & 0 & 1 \\
\hline \multirow[t]{2}{*}{$102=\{$} & 1 & 0 & -1 & 0 & 1 & 0 & -1 & 0 \\
\hline & \multicolumn{8}{|c|}{ FEC } \\
\hline $53=\{$ & 0 & 1 & 0 & 0 & -1 & 0 & -1 & -1 \\
\hline $102=$ & 0 & 1 & 1 & 0 & 1 & 0 & -1 & 0 \\
\hline
\end{tabular}
$K_{0}=53$ e $K_{1}=102$, a Joint NAF possui peso de Hamming conjunto 08, enquanto a FEC possui peso de Hamming 5.

O próximo exemplo usa a FEC juntamente com o Método rápido de Shamir. 
Método rápido de Shamir para computação $13 P+10 Q$

\begin{tabular}{|c|c|c|c|c|c|}
\hline $13=\{$ & 1 & 0 & 0 & -1 & -1 \\
\hline $10=\{$ & 0 & 1 & 0 & 1 & 0 \\
\hline
\end{tabular}

\begin{tabular}{|c|c|c|c|c|c|}
\hline Duplicação \{ & $\mathcal{O}$ & $2 P$ & $4 P+2 Q$ & $8 P+4 Q$ & $14 P+10 Q$ \\
\hline$P\{$ & $P$ & & & & $13 P+10 Q$ \\
\hline$Q\{$ & & $2 P+Q$ & & & \\
\hline$P+Q\{$ & & & & & \\
\hline$P-Q\{$ & & & & $7 P+5 Q$ & \\
\hline
\end{tabular}

Este método custa $\sim l$ duplicações e $\sim w$ adições, onde $l$ é o comprimento e $w$ o peso de Hamming conjunto da expansão. O Teorema 8.4 mostra que $w=l / 2$.

A FEC satisfaz a um conjunto de propriedades. A primeira propriedade a ser provada é a unicidade.

Teorema 8.1 (Unicidade da Forma Esparsa Conjunta). Um par $K_{0}, K_{1}$ de inteiros positivos possue no máximo uma FEC.

Demonstração. Suponha, ao contrário, que existam duas FEC.

$$
\left[\begin{array}{l}
K_{0} \\
K_{1}
\end{array}\right]=\left[\begin{array}{l}
u_{0, m}, \ldots, u_{0,1}, u_{0,0} \\
u_{1, m}, \ldots, u_{1,1}, u_{1,0}
\end{array}\right]=\left[\begin{array}{l}
w_{0, m}, \ldots, w_{0,1}, w_{0,0} \\
w_{1, m}, \ldots, w_{1,1}, w_{1,0}
\end{array}\right]
$$

Desde que as representações são diferentes, então $u_{i, j} \neq w_{i, j}$, para algum $i, j$. Seja $g$ o valor mínimo de $j$ em que as formas diferem. Seja então:

$$
\left[\begin{array}{c}
K_{0}^{\prime} \\
K_{1}^{\prime}
\end{array}\right]=\left[\begin{array}{l}
u_{0, m}, \ldots, u_{0, g+1}, u_{0, g} \\
u_{1, m}, \ldots, u_{1, g+1}, u_{1, g}
\end{array}\right]=\left[\begin{array}{l}
w_{0, m}, \ldots, w_{0, g+1}, w_{0, g} \\
w_{1, m}, \ldots, w_{1, g+1}, w_{1, g}
\end{array}\right]
$$

Desde que as expansões diferem em $j=g$, podemos supor que $u_{0, g} \neq$ $w_{0, g}$. Segue que $K_{0}^{\prime}$ é ímpar, pois de outra forma $u_{0, g}=w_{0, g}=0$. Portanto $u_{0, g}$ e $w_{0, g}$ devem ter valores no conjunto $\{ \pm 1\}$ (sem perda de generalidade, assumimos que $u_{0, g}=1$ e $w_{0, g}=-1$ ).

$$
\left[\begin{array}{c}
K_{0}^{\prime} \\
K_{1}^{\prime}
\end{array}\right]=\left[\begin{array}{c}
u_{0, m}, \ldots, u_{0, g+1},+1 \\
u_{1, m}, \ldots, u_{1, g+1}, u_{1, g}
\end{array}\right]=\left[\begin{array}{c}
w_{0, m}, \ldots, w_{0, g+1},-1 \\
w_{1, m}, \ldots, w_{1, g+1}, w_{1, g}
\end{array}\right]
$$

Como $K_{0}^{\prime}$ é ímpar, suponha que $K_{0}^{\prime} \equiv 1(\bmod 4)^{3}$. Desde que $u_{0, g}=1$, então $u_{0, g+1}=0\left(K_{0}^{\prime} \equiv 1(\bmod 4)\right)$. Desde que $w_{0, g}=-1$ então $w_{0, g+1}$ deve

\footnotetext{
${ }^{3} \mathrm{O}$ caso $K_{0}^{\prime} \equiv 3(\bmod 4)$ é similar
} 
ser ímpar, ou seja, $w_{0, g+1}= \pm 1{ }^{4}$. Pela (JS-2) temos $w_{0, g+1}=-1$. Segue da (JS-3) que $w_{1, g}=0$ e $w_{1, g+1}= \pm 1$ e portanto $K_{1}^{\prime} \equiv 2(\bmod 4)$. Segue também que $u_{1, g}=0$ e $u_{1, g+1}= \pm 1$.

Pela (JS-1), ambas as expansões tem um zero duplo na posição $g+2$. Portanto $u_{0, g+2}=u_{1, g+2}=w_{0, g+2}=w_{1, g+2}=0 \mathrm{e}$

$$
\left[\begin{array}{c}
K_{0}^{\prime} \\
K_{1}^{\prime}
\end{array}\right]=\left[\begin{array}{c}
u_{0, m}, \ldots, 0,0,+1 \\
u_{1, m}, \ldots, 0, \pm 1,0
\end{array}\right]=\left[\begin{array}{c}
w_{0, m}, \ldots, 0,-1,-1 \\
w_{1, m}, \ldots, 0, \pm 1,0
\end{array}\right]
$$

Esse resultado significa que $K_{0}^{\prime} \equiv 1(\bmod 8)$ desde que $\left[u_{0, g+2}, u_{0, g+1}, u_{0, g}\right]=$ $[0,0,+1]$, enquanto que $\left[w_{0, g+2}, w_{0, g+1}, w_{0, g}\right]=[0,-1,-1]$ é tal que $K_{0}^{\prime} \equiv 5$ $(\bmod 8)$. Esta contradição mostra que a suposição inicial foi errônea.

Como resultado do Teorema 8.1, podemos falar na FEC de dois inteiros.

\subsection{Computando a Forma Esparsa Conjunta}

O objetivo desta seção é descrever o algoritmo 8.2 que computa a FEC de Solinas. Inicialmente, descreveremos o algoritmo 8.1 (didático) que computa a FEC a partir das entradas inteiras $K_{0}$ e $K_{1}$. Deduzimos que foi a partir das idéias apresentadas neste algoritmo que o autor produziu o algoritmo 8.2 que computa a FEC a partir da forma reduzida de $K_{0}$ e $K_{1}$.

\footnotetext{
${ }^{4}$ caso contrário, se $w_{0, g+1}=0$, o valor inteiro das duas expressões nunca seriam idênticas, o que contraria a suposição inicial
} 


\section{Algoritmo 8.1 (Forma Esparsa Conjunta):}

Entrada: Números inteiros $K_{0}$ e $K_{1}$.

Saída: Uma expressão para $K_{0}$ e $K_{1}$ na Forma Esparsa Conjunta.

[1] $j \leftarrow 0$

[2] $d_{0} \leftarrow 0$ e $d_{1} \leftarrow 0$

[3] enquanto $\left(K_{0}+d_{0}+K_{1}+d_{1}>0\right)$

[4] fazer $L_{0} \leftarrow d_{0}+K_{0}$

[5] $\quad L_{1} \leftarrow d_{1}+K_{1}$

[6] para $i$ de 0 a 1

[7] fazer se $L_{i}$ é par

[8] então $u \leftarrow 0$

[9] $\quad \underline{\text { senão }} u \leftarrow L_{i} \operatorname{mods} 4$ (ver nota de rodapé na pág. 24)

[10] se $L_{i} \equiv \pm 3(\bmod 8)$ e $L_{1-i} \equiv 2(\bmod 4)$

[11] então $u \leftarrow-u$

[12]

$[13]$

[14]

[15]

$[16]$

[17]

[18] $j \leftarrow j+1$

$$
\begin{aligned}
& u_{i, j} \leftarrow u \\
& i \leftarrow i+1 \\
& \frac{\text { para } i \text { de } 0}{\text { fazer }} d_{i} \leftarrow\left(d_{i}+a_{i}-u_{i, j}\right) / 2 \\
& K_{i} \leftarrow\left\lfloor K_{i} / 2\right\rfloor \\
& i \leftarrow i+1
\end{aligned}
$$

Note que este algoritmo contém a lógica do algoritmo 3.5, que computa a expansão NAF de $K$. A diferença existente entre este algoritmo e 03.5 é a de que este possui a linhas adicionais (10 e 11) e a mesma operação é executada duas vezes.

Observando por este ângulo fica fácil perceber que a variável $L_{i}=K_{i}+d_{i}$ corresponde a variável $K$ do algoritmo 3.5, bem como notar que as linhas (15 e 16) efetuam na verdade a operação $L_{i} \leftarrow\left(L_{i}-u_{i, j}\right) / 2=\left(K_{i}+d_{i}-u_{i, j}\right) / 2=$ $\left\lfloor K_{i} / 2\right\rfloor+\left(a_{i}+d_{i}-u_{i, j}\right) / 2$, onde $a_{i}$ é o valor do bit menos significativo da expansão NAF de $K_{i}{ }^{5}$.

Vamos supor que não existissem as linhas (10 e 11). Assim sendo, cada passo deste algoritmo em que $L_{i}$ é ímpar, $L_{i}-u_{i, j}$ tornaria divisível por 4 e portanto $L_{i} \leftarrow\left(L_{i}-u_{i, j}\right) / 2$, linhas $(15$ e 16$)$, divisivel por 2 . Em outras palavras estaríamos computando a expansão NAF de $L_{i}$. Note que $d_{i} \leftarrow\left(a_{i}+d_{i}-u_{i, j}\right) / 2$ sempre será 0 , pois sempre ocorrerá a igualdade $a_{i}=u_{i, j}$.

Concluímos, portanto que $d_{i}$ armazena a mudança que poderá ocorrer no valor de $u_{i, j}$ quando as linhas (10 e 11) são inseridas. Reiteramos que o $u_{i, j}$ que seria obtido, caso não existissem as linhas (10 e 11) é aquela advinda da computação da Expansão NAF da entrada.

Porque as condições contidas na linha (10) produzem a FEC de $K_{0}$ e $K_{1}$ ?

\footnotetext{
${ }^{5} a_{i}=0$ (se $K_{i}$ é par) e $a_{i}=K_{i} \operatorname{mods} 4$ (se $K_{i}$ é ímpar)
} 
Para facilitar esta explicação consideramos a notação $L_{i, j}$ e $u_{j}$ representando, respectivamente, o valor de $L_{i}$ e $u$ no passo $j$ do algoritmo. Vamos considerar também $\gamma$ e $\delta$ como variáveis pertencentes ao conjunto $\{ \pm 1\}$

Vamos supor que $L_{0, j} \equiv \pm 3(\bmod 8)$ e $L_{1, j} \equiv 2(\bmod 4)$ e verifiquemos as saída $u_{i, j}$ em cada passo:

- Estado $j$

- $(i=0): u_{0, j}=-\gamma$ e não $u_{0, j}=\gamma$, que deveria ocorrer para produzir a expansão NAF de $L_{0, j}$. Desta forma $L_{0, j}-u_{0, j}=$ $L_{0, j}+\gamma$ não será divisível por 4 e $L_{0, j+1} \leftarrow\left(L_{0, j}+\gamma\right) / 2$ será tal que $L_{0, j+1} \operatorname{mods} 4=-\gamma$.

- $(i=1): u_{1, j}=0$ e $L_{1, j+1} \leftarrow\left(L_{1, j}\right) / 2$ ímpar.

- Estado $j+1$

$-(i=0): u_{0, j+1}=-\gamma$ e $L_{0, j+2} \leftarrow\left(L_{0, j+1}+\gamma\right) / 2$ é par.

- $(i=1): u_{0, j+1}=\delta$, que deveria ocorrer para produzir a expansão NAF de $L_{1, j+1}$. Desta forma $L_{0, j+1}-u_{1, j+1}=L_{0, j}-\delta$ será divisível por 4 e $L_{1, j+2} \leftarrow\left(L_{0, j+1}-\delta\right) / 2$ par.

- Estado $j+2$

$-(i=0): u_{0, j+2}=0$

$-(i=1): u_{0, j+2}=0$

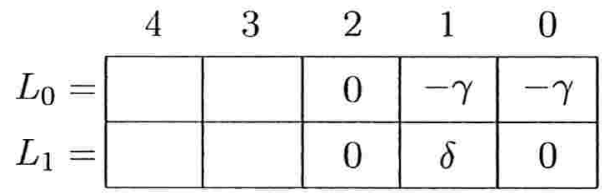

Portanto, a modificação introduzida pelas linhas (10 e 11 ) permite a obtenção da FEC da entrada.

O exemplo 8.3 contém a simulação do funcionamento do algoritmo $8.1 \mathrm{se}$ visualizarmos como entrada os inteiros $K_{0}$ e $K_{1}$.

No algoritmo 8.1 as operações que são executadas sobre os inteiros $K_{i} \mathrm{e}$ $L_{i}(i \in\{0,1\})$ provém da análise dos 3 bits menos significativos, sendo que a operação $\left\lfloor K_{i} / 2\right\rfloor$ desloca o bit menos significativo de $K_{i}$ para a esquerda. Assim sendo, embora o algoritmo seja expresso em termos da aritmética de inteiros, na verdade ele executa operações sobre as entradas binárias de $K_{i}$. Portanto, podemos modificar o algoritmo 8.1, produzindo o algoritmo 8.2 que tem como entrada uma expansão binária conjunta reduzida de $K_{0}$ e $K_{1}$. 
Uma expansão binária com sinal

$$
\left[K_{0}\right]=\left[u_{0, m}, \ldots, u_{0,1}, u_{0,0}\right]
$$

é dita ser reduzida se $u_{j-1} u_{j} \neq-1$ para todo $j>0$. Uma expressão binária com sinal não reduzida contém mais bits do que o necessário para expressálo. A expressão binária simples e a expressão NAF binária são expressões binárias reduzidas.

Uma expansão binária conjunta com sinal (8.2) é dita ser reduzida se consiste de duas expansões binárias com sinal reduzidas. Portanto a FEC é reduzida pela (JS-2).

\section{Algoritmo 8.2 (Forma Esparsa Conjunta):}

Entrada: Uma expansão binária com sinal conjunta reduzida

$$
\left[\begin{array}{c}
K_{0} \\
K_{1}
\end{array}\right]=\left[\begin{array}{l}
e_{0, m-1}, \ldots, e_{0,2}, e_{0,1}, e_{0,0} \\
e_{1, m-1}, \ldots, e_{1,2}, e_{1,1}, e_{1,0}
\end{array}\right] .
$$

Saída: Uma expressão (8.2) para $K_{0}, K_{1}$ na FEC.

$$
\begin{aligned}
& \text { [1] } a_{0} \leftarrow e_{0,0} ; b_{0} \leftarrow e_{0,1} ; c_{0} \leftarrow e_{0,2} ; d_{0} \leftarrow 0 \\
& \text { [2] } a_{1} \leftarrow e_{1,0} ; b_{1} \leftarrow e_{1,1} ; c_{1} \leftarrow e_{1,2} ; d_{1} \leftarrow 0 \\
& \text { [3] para } j=0 \text { até } m \\
& \text { [4] fazer para } i=0 \text { até } 1 \\
& \text { [5] } \quad \underline{\text { fazer }} \text { se } d_{i} \equiv a_{i}(\bmod 2) \\
& \text { [6] então } u \leftarrow 0 \\
& \text { [7] } \quad \underline{\text { senão }} u \leftarrow\left(d_{i}+2 b_{i}+a_{i}\right) \operatorname{mods} 4 \quad \text { (ver rodapé pág. 24) } \\
& \text { [8] } \quad \text { se } d_{i}+4 c_{i}+2 b_{i}+a_{i} \equiv \pm 3(\bmod 8) \\
& \text { [9] } \mathrm{e} \\
& \text { [10] } \quad d_{1-i}+2 b_{1-i}+a_{1-i} \equiv 2(\bmod 4) \\
& \text { [11] } \quad \text { então } u \leftarrow-u \\
& \begin{array}{ll}
\text { [12] } & u_{i, j} \leftarrow u \\
\text { [13] } & R_{i, j} \leftarrow\left(d_{i}, c_{i}, b_{i}, a_{i}\right)
\end{array} \\
& \text { [14] } S_{j} \leftarrow\left[\begin{array}{l}
R_{0, j}=d_{0}, c_{0}, b_{0}, a_{0} \\
R_{1, j}=d_{1}, c_{1}, b_{1}, a_{1}
\end{array}\right] \\
& \text { [15] } \quad \text { para } i=0 \text { até } 1 \\
& \text { [16] } \quad \text { fazer } d_{i} \leftarrow\left(d_{i}+a_{i}-u_{i, j}\right) / 2 \\
& \text { [17] } \quad a_{i} \leftarrow b_{i} ; b_{i} \leftarrow c_{i} ; c_{i} \leftarrow e_{i, j+3}
\end{aligned}
$$

Os valores $R_{i, j}$ e $S_{j}$ não fazem parte do algoritmo e foram incluídos somente para estabelecer notação para a prova que será descrita.

Por convenção $e_{i, j}=0$ para $j \geq m$.

Pode ser facilmente verificado que $d_{i} \in\{0, \pm 1\}$ e que a linha (5) do algoritmo verifica se $L_{i}$ é par.

O exemplo 8.3 também simula o funcionamento do algoritmo 8.2 se visualizarmos como entrada a expansão binária conjunta reduzida de $K_{0}$ e $K_{1}$. 
Exemplo 8.3. Funcionamento dos algoritmos 8.1 e 8.2.

\begin{tabular}{|c|c|c|c|c|c|c|c|c|c|}
\hline \multirow{3}{*}{$\begin{array}{r}K_{0}=53\{ \\
K_{1}=102\{\end{array}$} & & & & & \multicolumn{4}{|c|}{$J-1$} & \multirow{3}{*}{$\begin{array}{l}\} d_{0}=0 \rightarrow L_{0}=53 \\
\} d_{1}=0 \rightarrow L_{1}=102\end{array}$} \\
\hline & 0 & 1 & 0 & -1 & 0 & 1 & 0 & 1 & \\
\hline & 1 & 0 & -1 & 0 & 1 & 0 & -1 & 0 & \\
\hline & & & & & & & $J$ & & \\
\hline$K_{0}=26\{$ & 0 & 1 & 0 & -1 & 0 & 1 & 0 & -1 & \}$d_{0}=1 \rightarrow L_{0}=27$ \\
\hline$K_{1}=51\{$ & 1 & 0 & -1 & 0 & 1. & 0 & -1 & 0 & \}$d_{1}=0 \rightarrow L_{1}=51$ \\
\hline$K_{0}=13\{$ & 0 & 1 & 0 & -1 & 0 & 1 & -1 & -1 & \}$d_{0}=1 \rightarrow L_{0}=14$ \\
\hline$K_{1}=26\{$ & 1 & 0 & -1 & 0 & 1 & 0 & -1 & 0 & \}$d_{1}=0 \rightarrow L_{1}=26$ \\
\hline$K_{0}=6\{$ & 0 & 1 & 0 & -1 & 0 & 0 & -1 & -1 & \}$d_{0}=1 \rightarrow L_{0}=7$ \\
\hline$K_{1}=13\{$ & 1 & 0 & -1 & 0 & 1 & 0 & -1 & 0 & \}$d_{1}=0 \rightarrow L_{1}=13$ \\
\hline$K_{0}=3\{$ & 0 & 1 & 0. & -1 & -1 & 0 & -1 & -1 & \}$d_{0}=1 \rightarrow L_{0}=4$ \\
\hline$K_{1}=6\{$ & 1 & 0 & -1 & 0 & 1 & 0 & -1 & 0 & \}$d_{1}=0 \rightarrow L_{1}=6$ \\
\hline$K_{0}=2\{$ & 0 & 1 & 0 & 0 & -1 & 0 & -1 & -1 & \}$d_{0}=0 \rightarrow L_{0}=2$ \\
\hline$K_{1}=3\{$ & 1 & 0 & -1 & 0 & 1 & 0 & -1 & 0 & \}$d_{1}=0 \rightarrow L_{1}=3$ \\
\hline$K_{0}=1\{$ & 0 & 1 & 0 & 0 & -1 & 0 & -1 & -1 & \}$d_{0}=0 \rightarrow L_{0}=1$ \\
\hline$K_{1}=2\{$ & 1 & $\mathbf{0}$ & 1 & 0 & 1 & 0 & -1 & 0 & \}$d_{1}=-1 \rightarrow L_{1}=1$ \\
\hline$K_{0}=0\{$ & $\mathbf{0}$ & 1 & 0 & 0 & -1 & 0 & -1 & -1 & \}$d_{0}=0 \rightarrow L_{0}=0$ \\
\hline$K_{1}=1\{$ & 1. & 1 & 1 & 0 & 1 & 0 & -1 & 0 & \}$d_{1}=-1 \rightarrow L_{1}=0$ \\
\hline$K_{0}=0\{$ & 0 & 1 & 0 & 0 & -1 & 0 & -1 & -1 & \}$d_{0}=0 \rightarrow L_{0}=0$ \\
\hline$K_{1}=0\{$ & 0 & 1 & 1 & 0 & 1 & 0 & -1 & 0 & \}$d_{1}=0 \rightarrow L_{1}=0$ \\
\hline
\end{tabular}

\subsection{Corretude do algoritmo}

Descreveremos a prova de que o algoritmo 8.2 produz a FEC da entrada.

Como as operações são executadas sobre os 3 últimos bits da expansão de entrada, vamos considerar $K_{i, j}=4 c_{i}+2 b_{i}+a_{i}$ e $L_{i, j}=d_{i}+K_{i, j}$

Os vetores $S_{j}$ são denominados estados do algoritmo. A $j$-ésima operação do loop tem como entrada o estado $S_{j-1}$ ( que produz saída $\left(u_{0, j-1}, u_{1, j-1}\right)$ ) e troca o estado para $S_{j}$.

Existem 51 possíveis valores para cada $R_{i, j} \leftarrow\left(d_{i}, c_{i}, b_{i}, a_{i}\right)$, isto porque algumas combinações não ocorrem desde que $e_{i, j}$ é reduzida, ou seja, $u_{j-1}, u_{j} \neq$ -1 .

Para cada $R_{i, j}$, temos:

$$
\begin{aligned}
K_{i, j} & =4 c_{i}+2 b_{i}+a_{i} \\
L_{i, j} & =d_{i}+K_{i, j} .
\end{aligned}
$$

Os 51 possíveis valores de $R_{i, j}$ são 


\begin{tabular}{lllll}
\hline \multicolumn{5}{c}{$R_{i, j}$} \\
\hline$(0000)$ & $(\overline{1} 001)$ & $(1111)$ & $(100 \overline{1})$ & $(\overline{1} \overline{1} \overline{1} \overline{1})$ \\
$(0001)$ & $(1000)$ & $(\overline{1} 010)$ & & \\
$(0010)$ & $(1001)$ & $(\overline{1} 011)$ & $(\overline{1} 10 \overline{1})$ & \\
$(0011)$ & $(1010)$ & $(010 \overline{1})$ & $(\overline{1} 100)$ & \\
$(0100)$ & $(1011)$ & $(\overline{1} 101)$ & $(110 \overline{1})$ & \\
$(0101)$ & $(1100)$ & $(\overline{1} 110)$ & & \\
$(0110)$ & $(1101)$ & $(\overline{1} 111)$ & & \\
$(0111)$ & $(1110)$ & & & \\
$(000 \overline{1})$ & $(\overline{1} 000)$ & $(10 \overline{1} 0)$ & & \\
$(00 \overline{1} 0)$ & $(\overline{1} 00 \overline{1})$ & $(10 \overline{1} \overline{1})$ & $(1 \overline{1} 01)$ & \\
$(00 \overline{1} \overline{1})$ & $(\overline{1} 0 \overline{1} 0)$ & $(1 \overline{1} 00)$ & $(0 \overline{1} 01)$ & \\
$(0 \overline{1} 00)$ & $(\overline{1} 0 \overline{1} \overline{1})$ & $(1 \overline{1} 0 \overline{1})$ & $(\overline{1} \overline{1} 01)$ & \\
$(0 \overline{1} 0 \overline{1})$ & $(\overline{1} \overline{1} 00)$ & $(1 \overline{1} \overline{1} 0)$ & & \\
$(0 \overline{1} \overline{1} 0)$ & $(\overline{1} \overline{1} 0 \overline{1})$ & $(1 \overline{1} \overline{1} \overline{1})$ & & \\
$(0 \overline{1} \overline{1} \overline{1})$ & $(\overline{1} \overline{1} \overline{1} 0)$ & & & \\
\hline
\end{tabular}

Da tabela acima, concluímos que $L_{i, j}$ pode assumir valores inteiros no intervalo $(-8$ a 8$)$.

Em seguida os estados são agrupados de acordo com os valores de $L_{i, j}$

\begin{tabular}{|c|c|}
\hline \multirow{2}{*}{$\mathbf{S}_{\mathbf{j}}$} & $R_{\mathbf{0}, j}$ \\
& $R_{1, j}$ \\
\hline \multirow{2}{*}{$\mathbf{C}$} & $L_{i, j} \operatorname{par}$ \\
\cline { 2 - 2 } & $L_{i, j} \operatorname{par}$ \\
\hline \multirow{2}{*}{$\mathbf{D}$} & $L_{i, j} \equiv 0(\bmod 4)$ \\
\cline { 2 - 2 } & $L_{i, j} \operatorname{mpar}$ \\
\hline \multirow{2}{*}{$\mathbf{E}$} & $L_{i, j} \equiv 2(\bmod 4)$ \\
\cline { 2 - 2 } & $L_{i, j} \equiv \pm 1(\bmod 8)$ \\
\hline \multirow{2}{*}{$\mathbf{F}$} & $L_{i, j} \equiv 2(\bmod 4)$ \\
\cline { 2 - 2 } & $L_{i, j} \equiv \pm 3(\bmod 8)$ \\
\hline \multirow{2}{*}{$\mathbf{G}$} & $L_{i, j} \operatorname{mpar}$ \\
\cline { 2 - 2 } & $L_{i, j} \equiv 0(\bmod 4)$ \\
\hline \multirow{2}{*}{$\mathbf{H}$} & $L_{i, j} \equiv \pm 1(\bmod 8)$ \\
\cline { 2 - 2 } & $L_{i, j} \equiv 2(\bmod 4)$ \\
\hline \multirow{2}{*}{$\mathbf{J}$} & $L_{i, j} \equiv \pm 3(\bmod 8)$ \\
\cline { 2 - 2 } & $L_{i, j} \equiv 2(\bmod 4)$ \\
\hline \multirow{2}{*}{$\mathbf{K}$} & $L_{i, j} \operatorname{mpar}$ \\
\cline { 2 - 2 } & $L_{i, j} \operatorname{mpar}$ \\
\hline
\end{tabular}

Apresentamos, como ilustração, estados obtidos para o exemplo 8.3: 


$$
\begin{array}{cccccccc}
\mathbf{J} & \mathbf{K} & \mathbf{C} & \mathbf{K} & \mathbf{C} & \mathbf{F} & \mathbf{K} & \mathbf{C} \\
{\left[\begin{array}{c}
5 \\
-2
\end{array}\right]} & {\left[\begin{array}{l}
3 \\
3
\end{array}\right]} & {\left[\begin{array}{c}
-2 \\
2
\end{array}\right]} & {\left[\begin{array}{c}
-1 \\
-3
\end{array}\right]} & {\left[\begin{array}{c}
4 \\
-2
\end{array}\right]} & {\left[\begin{array}{l}
2 \\
3
\end{array}\right]} & {\left[\begin{array}{l}
1 \\
1
\end{array}\right]} & {\left[\begin{array}{l}
0 \\
0
\end{array}\right]}
\end{array}
$$

Existe um conjunto de regras de transição entres estados, abaixo discriminadas. Na seção 8.5.1 forneceremos a prova da corretude das mesmas.

1. Se $\left(\begin{array}{c}L_{i, j} \equiv 0(\bmod 4) \\ L_{1-i . j} \text { impar }\end{array}\right)$ então $\left(\begin{array}{c}L_{i, j+1} \operatorname{par} \\ -\end{array}\right)$

2. Se $\left(\begin{array}{c}L_{i, j} \equiv 2(\bmod 4) \\ L_{1-i, j} \text { ímpar }\end{array}\right)$ então $\left(\begin{array}{c}L_{i, j+1} \text { ímpar } \\ -\end{array}\right)$

3. Se $\left(\begin{array}{c}L_{i, j} \text { ímpar } \\ L_{1-i, j} \not \equiv 2(\bmod 4)\end{array}\right)$ então $\left(\begin{array}{c}L_{i, j+1} \text { par } \\ -\end{array}\right)$

4. Se $\left(\begin{array}{c}L_{i, j} \equiv \pm 1(\bmod 4) \\ L_{1-i, j} \equiv 2(\bmod 4)\end{array}\right) \operatorname{então}\left(\begin{array}{c}L_{i, j+1} \equiv 0(\bmod 4) \\ -\end{array}\right)$

5. Se $\left(\begin{array}{c}L_{i, j} \equiv \pm 3(\bmod 4) \\ L_{1-i, j} \equiv 2(\bmod 4)\end{array}\right)$ então $\left(\begin{array}{c}L_{i, j+1} \text { ímpar } \\ -\end{array}\right)$

Como resultado destas regras, obtemos os valores de $S_{j+1}$ para cada $S_{j}$ dados na Tabela 8.2. As possíveis saídas $\left\langle u_{i, j}, u_{1-i, j}\right\rangle$ para cada estado são também fornecidos pois serão úteis.

A entrada $*$ indica que qualquer estado é um possível sucessor para o estado $C$. Em todos os outros casos, o estado $S_{j+1}$ é determinado por $S_{j}$. 


\begin{tabular}{|c|c|c|c|}
\hline$S_{j}$ & Regras & $u_{i, j}, u_{1-i, j}$ & $S_{j+1}$ \\
\hline \multirow{2}{*}{ C } & \multirow{2}{*}{ * } & 0 & \multirow{2}{*}{ * } \\
\hline & & 0 & \\
\hline \multirow{2}{*}{ D } & regra 1 & 0 & \multirow{2}{*}{ C } \\
\hline & regra 3 & \pm 1 & \\
\hline \multirow{2}{*}{$\mathrm{E}$} & regra 2 & 0 & \multirow{2}{*}{ G } \\
\hline & regra 4 & \pm 1 & \\
\hline \multirow{2}{*}{ F } & regra 2 & 0 & \multirow{2}{*}{ K } \\
\hline & regra 5 & \pm 1 & \\
\hline \multirow{2}{*}{ G } & regra 3 & \pm 1 & \multirow{2}{*}{ C } \\
\hline & regra1 & 0 & \\
\hline \multirow{2}{*}{$\mathbf{H}$} & regra 4 & \pm 1 & \multirow{2}{*}{ D } \\
\hline & regra 2 & 0 & \\
\hline \multirow{2}{*}{$\mathbf{J}$} & regra 5 & \pm 1 & \multirow{2}{*}{ K } \\
\hline & regra 2 & 0 & \\
\hline \multirow{2}{*}{ K } & regra 3 & \pm 1 & \multirow{2}{*}{ C } \\
\hline & regra 3 & \pm 1 & \\
\hline
\end{tabular}

Tabela 8.2: Transição entre estados

A figura 8.1 contém um diagrama de transição entre os estados.

Apresentaremos, novamente, como ilustração, a transição entre os estados no exemplo 8.3, juntamente com as saídas $\left\langle u_{i, j}, u_{1-i, j}\right\rangle$ para cada estado:

$$
\begin{aligned}
& \begin{array}{cccccccc}
\mathbf{J} & \mathbf{K} & \mathbf{C} & \mathbf{K} & \mathbf{C} & \mathbf{F} & \mathbf{K} & \mathbf{C} \\
{\left[\begin{array}{c}
5 \\
-2
\end{array}\right]} & {\left[\begin{array}{l}
3 \\
3
\end{array}\right]} & {\left[\begin{array}{c}
-2 \\
2
\end{array}\right]} & {\left[\begin{array}{c}
-1 \\
-3
\end{array}\right]} & {\left[\begin{array}{c}
4 \\
-2
\end{array}\right]} & {\left[\begin{array}{l}
2 \\
3
\end{array}\right]} & {\left[\begin{array}{l}
1 \\
1
\end{array}\right]} & {\left[\begin{array}{l}
0 \\
0
\end{array}\right]}
\end{array} \\
& {\left[\begin{array}{c}
a_{i} \\
a_{1-i}
\end{array}\right] \quad\left[\begin{array}{l}
1 \\
0
\end{array}\right] \quad\left[\begin{array}{c}
0 \\
-1
\end{array}\right] \quad\left[\begin{array}{l}
1 \\
0
\end{array}\right] \quad\left[\begin{array}{l}
0 \\
1
\end{array}\right] \quad\left[\begin{array}{c}
-1 \\
0
\end{array}\right] \quad\left[\begin{array}{c}
0 \\
-1
\end{array}\right] \quad\left[\begin{array}{l}
1 \\
0
\end{array}\right] \quad\left[\begin{array}{l}
0 \\
1
\end{array}\right]} \\
& {\left[\begin{array}{c}
u_{i, j-1} \\
u_{1-i, j-1}
\end{array}\right] \quad\left[\begin{array}{c}
-1 \\
0
\end{array}\right] \quad\left[\begin{array}{c}
-1 \\
-1
\end{array}\right] \quad\left[\begin{array}{l}
0 \\
0
\end{array}\right] \quad\left[\begin{array}{c}
-1 \\
1
\end{array}\right] \quad\left[\begin{array}{l}
0 \\
0
\end{array}\right] \quad\left[\begin{array}{l}
0 \\
1
\end{array}\right] \quad\left[\begin{array}{l}
1 \\
1
\end{array}\right] \quad\left[\begin{array}{l}
0 \\
0
\end{array}\right]}
\end{aligned}
$$

Finalmente, é útil combinar os 8 estados acima em três, como se segue 


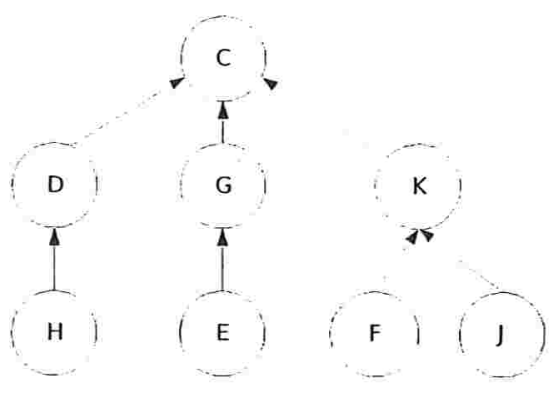

Figura 8.1: Fluxo da transição entre os 8 estados do Algoritmo 8.2

\begin{tabular}{|c|c|c|}
\multicolumn{1}{c|}{$S_{j} U_{0, j}=U_{1, j}=0$} & $S_{j+1}$ \\
\hline $\begin{array}{c}\mathrm{A} \\
(\mathrm{E}-\mathrm{F}-\mathrm{H} \text { ou J })\end{array}$ & Não & $\mathrm{B}$ \\
\hline $\begin{array}{c}\mathrm{B} \\
(\mathrm{D}-\mathrm{G} \text { ou K })\end{array}$ & Não & $\mathrm{C}$ \\
\hline $\begin{array}{c}\mathrm{C} \\
(\mathrm{C})\end{array}$ & Sim & $\mathrm{A}, \mathrm{B}$ ou C \\
\hline
\end{tabular}

Teorema 8.2. O Algoritmo 8.2 sempre tem como saída a FEC de sua entrada.

Demonstração. Verifica-se diretamente que a expansão (8.2) produzida pelo algoritmo é de fato uma expansão binária com sinal conjunta de $K_{0}$ e $K_{1}$. Resta provar que esta expansão satisfaz as propriedades (JS-1), (JS-2) e (JS-3).

(JS-1) De qualquer três posições consecutivas, no mínimo uma é um zero duplo (uma coluna zero). Em outras palavras, para qualquer $i$ e $j$ positivo, temos $U_{i, j+k}=U_{1-i, j+k}=0$ para $k=0$ ou $k= \pm 1$.

Esta condição é equivalente a asserção que, para todo $j$, no mínimo um dos estados $S_{j-1}, S_{j}$, e $S_{j+1}$ está no estado $C$. Suponha que $S_{j-1}$ não está no estado $C$; então o estado dele é $B$ (tal que $S_{j}$ está no estado $C$ ) ou $A$ (tal que $S_{j+1}$ está no estado $C$ ).

(JS-2) Termos adjacentes não possuem sinais opostos. Em outras palavras, nunca ocorre o caso em que $u_{i, j+1}, u_{i, j}=-1$.

Suponha que $u_{0, j} \neq 0$ e $u_{0, j+1} \neq 0$. Então segue da Tabela 8.2 que $S_{j}$ está no estado $J$ e que $S_{j+1}$ está no estado $K$. É direto computar $u_{0, j+1}$ e verificar que é igual a $u_{0, j}$ em cada dos possíveis casos para $R_{0, j}$ que compreende o estado $J$. Análogo procedimento obviamente aplica-se para $u_{1, j}$ e $u_{1, j+1}$. 

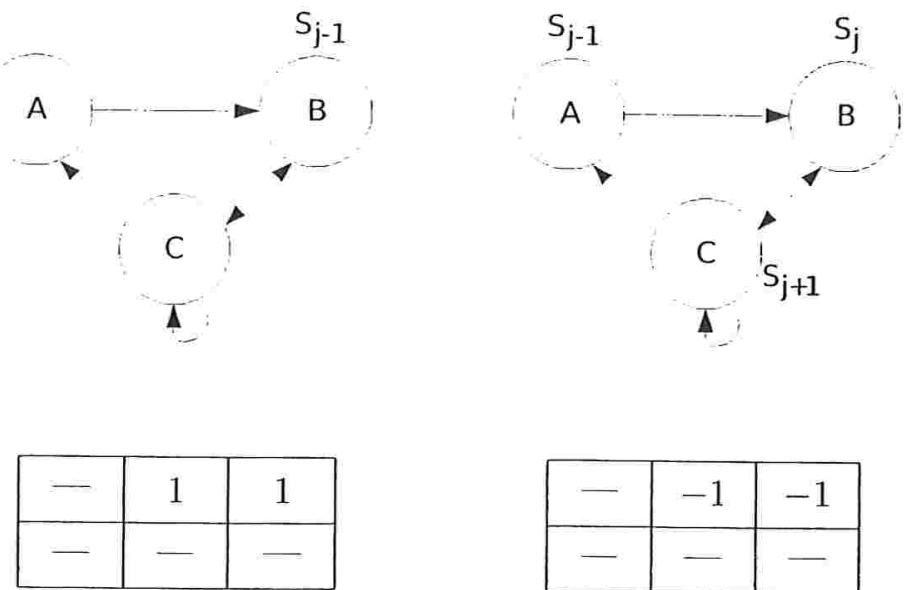

\begin{tabular}{|l|l|l|}
\hline- & -1 & -1 \\
\hline- & - & - \\
\hline
\end{tabular}

(JS-3) Se $u_{i, j+1} u_{i, j} \neq 0$ então $u_{1-i, j+1}= \pm 1$ e $u_{1-i, j}=0$.

Suponha que $u_{0, j+1} u_{0, j} \neq 0$, tal que $S_{j}$ está no estado $J$ e que $S_{j+1}$ está no estado $K$. Então a Tabela 8.2 mostra que $u_{1, j}=0$ e $u_{1, j+1}= \pm 1$. O caso $u_{1, j+1} u_{1, j} \neq 0$ é similar.

\begin{tabular}{|c|c|c|}
\hline- & -1 & -1 \\
\hline- & \pm 1 & 0 \\
\hline
\end{tabular}

ou

\begin{tabular}{|c|c|c|}
\hline- & 1 & 1 \\
\hline- & \pm 1 & 0 \\
\hline
\end{tabular}

\subsubsection{Corretude das regras de transição entre estados}

Nesta seção propomos demostrar a corretude das regras de transição que forma apresentadas referente ao algoritmo 8.2.

1. Se $\left(\begin{array}{c}L_{i, j} \equiv 0(\bmod 4) \\ L_{1-i, j} \text { ímpar }\end{array}\right)$ então $\left(\begin{array}{c}L_{i, j+1} \text { par } \\ -\end{array}\right)$.

Por hipótese, $\left(4 c_{i}+2 b_{i}+a_{i}+d_{i}\right) \equiv 0(\bmod 4) . \operatorname{Logo}\left(2 b_{i}+a_{i}+d_{i}\right) \equiv 0$ $(\bmod 4)$.

Se $b_{i}=0$, então $\left(a_{i}, d_{i}\right) \in\{(0,0) ;(-1,1) ;(1,-1)\} \Rightarrow a_{i} \equiv d_{i}(\bmod 2) \Rightarrow$ $u=u_{i, j}=0$ (linhas 6 e 12) e novo $\left(d_{i}\right)=0$ (linha 16).

Se $b_{i}=1$ ou $b_{i}=-1$, então $\left(a_{i}, d_{i}\right) \in\{(1,1) ;(-1,-1)\} \Rightarrow a_{i} \equiv d_{i}$ $(\bmod 2) \Rightarrow u=u_{i, j}=0($ linhas 6 e 12$)$ e novo $\left(d_{i}\right)=1$ ou -1 (linha $16)$.

Devemos provar que $\left(4 e_{i, j+3}+2 c_{i}+b_{i}+\operatorname{novo}\left(d_{i}\right)\right) \equiv 0(\bmod 2) . \operatorname{Logo}$ $\left(b_{i}+\operatorname{novo}\left(d_{i}\right)\right) \equiv 0(\bmod 2)$, que se mantém para todos os valores de $b_{i}$. 
2. Se $\left(\begin{array}{c}L_{i, j} \equiv 2(\bmod 4) \\ L_{1-i, j} \text { ímpar }\end{array}\right)$ então $\left(\begin{array}{c}L_{i, j+1} \text { ímpar } \\ -\end{array}\right)$.

Por hipótese $\left(4 c_{i}+2 b_{i}+a_{i}+d_{i}\right) \equiv 2(\bmod 4) . \operatorname{Logo}\left(2\left(b_{i}-1\right)+a_{i}+d_{i}\right) \equiv$ $0(\bmod 4)$.

Se $b_{i}=1$ ou $b_{i}=-1$, então $\left(a_{i}, d_{i}\right) \in\{(0,0) ;(-1,1) ;(1,-1)\} \Rightarrow a_{i} \equiv$ $d_{i}(\bmod 2) \Rightarrow u=u_{i, j}=0 \cdot(\operatorname{linhas} 6$ e 12$)$ e novo $\left(d_{i}\right)=0$ (linha 16$)$.

Se $b_{i}=0$, então $\left(a_{i}, d_{i}\right) \in\{(1,1) ;(-1,-1)\} \Rightarrow a_{i} \equiv d_{i}(\bmod 2) \Rightarrow u=$ $u_{i, j}=0$ (linhas 6 e 12) e novo $\left(d_{i}\right)=1$ ou -1 (linha 16$)$.

Devemos provar que $\left(4 e_{i, j+3}+2 c_{i}+b_{i}+\operatorname{novo}\left(d_{i}\right)\right) \equiv 1(\bmod 2)$. Logo $\left(b_{i}+\operatorname{novo}\left(d_{i}\right)\right) \equiv 1(\bmod 2)$, que se mantém para todos os valores de $b_{i}$.

3. Se $\left(\begin{array}{c}L_{i, j} \text { ímpar } \\ L_{1-i, j} \neq \equiv 2(\bmod 4)\end{array}\right)$, então $\left(\begin{array}{c}L_{i, j+1} \text { par } \\ -\end{array}\right)$.

Por hipótese $\left(4 c_{i}+2 b_{i}+a_{i}+d_{i}\right) \equiv 1(\bmod 2) . \quad$ Logo $\left(a_{i}+d_{i}\right) \equiv 1$ $(\bmod 2) \Rightarrow\left(a_{i}, d_{i}\right) \in\{(1,0) ;(-1,0) ;(0,1) ;(0,-1)\}$. Como $u=u_{i, j}=$ $\left(\left(a_{i}+d_{i}\right)+2 b_{i}\right)$ mods 4 (linhas 7 e 12$)$, temos que

Se $b_{i}=0$, então $u_{i, j}=a_{i}+d_{i} \in\{1,-1\}$ e novo $\left(d_{i}\right)=0$

Se $b_{i}=1$ ou $b_{i}=-1$, então $u_{i, j}=\left(a_{i}+d_{i} \pm 2\right) \operatorname{mods} 4=-\left(a_{i}+d_{i}\right) \mathrm{e}$ $\operatorname{novo}\left(d_{i}\right)=a_{i}+d_{i}$

Devemos provar que $\left(4 e_{i, j+3}+2 c_{i}+b_{i}+\operatorname{novo}\left(d_{i}\right)\right) \equiv 0(\bmod 2) . \operatorname{Logo}$ $\left(b_{i}+\operatorname{novo}\left(d_{i}\right)\right) \equiv 0(\bmod 2)$, que se mantém para todos os valores de $b_{i}$.

4. Se $\left(\begin{array}{c}L_{i, j} \equiv \pm 1(\bmod 4) \\ L_{1-i, j} \equiv 2(\bmod 4)\end{array}\right)$, então $\left(\begin{array}{c}L_{i, j+1} \equiv 0(\bmod 4) \\ -\end{array}\right)$.

Por hipótese $\left(4 c_{i}+2 b_{i}+a_{i}+d_{i}-1\right) \equiv 0(\bmod 4)$ ou $\left(4 c_{i}+2 b_{i}+a_{i}+\right.$ $\left.d_{i}+1\right) \equiv 0(\bmod 4)$. Portanto $\left(2 b_{i}+a_{i}+d_{i}-1\right) \equiv 0(\bmod 4)$ ou $\left(2 b_{i}+a_{i}+d_{i}+1\right) \equiv 0(\bmod 4)$.

Se $b_{i}=0$ então $\left(a_{i}+d_{i}-1\right) \equiv 0(\bmod 4)$ ou $\left(a_{i}+d_{i}+1\right) \equiv 0$ $(\bmod 4) \Rightarrow\left(a_{i}, d_{i}\right) \in\{(1,0) ;(0,1) ;(-1,0) ;(0,-1)\} \Rightarrow\left(a_{i}+d_{i}\right) \equiv 1$ $(\bmod 2) \Rightarrow u=u_{i, j}=a_{i}+d_{i} \in\{1,-1\}\left(\right.$ linhas 7 e 12) e novo $\left(d_{i}\right)=0$ (linha 16).

Se $b_{i}=1$ então $\left(a_{i}+d_{i}+1\right) \equiv 0(\bmod 4)$ ou $\left(a_{i}+d_{i}+3\right) \equiv 0$ $(\bmod 4) \Rightarrow\left(a_{i}, d_{i}\right) \in\{(0,-1) ;(-1,0)\} \Rightarrow u=u_{i, j}=d_{i}+2 b_{i}+a_{i}=1$ (linhas 7 e 12) e novo $\left(d_{i}\right)=-1$ (linha 16).

Se $b_{i}=-1$ então $\left(a_{i}+d_{i}-3\right) \equiv 0(\bmod 4)$ ou $\left(a_{i}+d_{i}-1\right) \equiv(\bmod 4) \Rightarrow$ $\left(a_{i}, d_{i}\right)=\{(0,1) ;(1,0)\} \Rightarrow u=u_{i j}=d_{i}+2 b_{i}+a_{i}=-1$ (linhas 7 e 12$)$ e novo $\left(d_{i}\right)=1$ (linha 16). 
Devemos provar que $\left(4 e_{i, j+3}+2 c_{i}+b_{i}+\operatorname{novo}\left(d_{i}\right)\right) \equiv 0(\bmod 4) \Rightarrow$ $\left(2 c_{i}+b_{i}+\operatorname{novo}\left(d_{i}\right)\right) \equiv 1(\bmod 4)$.

Podemos observar pela tese que nos casos $b_{i}=1$ e $b_{i}=-1$ não existe nenhum valor possível para $c_{i}$, logo eles não ocorrem na hipótese.

$\mathrm{O}$ caso em que $b_{i}=0$, temos, pela tese, que $c_{i}=0$, assim sendo somente este caso pode ocorrer na hipótese e a provạ se segue.

5. Se $\left(\begin{array}{c}L_{i, j} \equiv \pm 3(\bmod 4) \\ L_{1-i, j} \equiv 2(\bmod 4)\end{array}\right)$, então $\left(\begin{array}{c}L_{i, j+1} \text { ímpar } \\ -\end{array}\right)$.

Por hipótese $\left(4 c_{i}+2 b_{i}+a_{i}+d_{i}-3\right) \equiv 0(\bmod 4)$ ou $\left(4 c_{i}+2 b_{i}+\right.$ $\left.a_{i}+d_{i}+3\right) \equiv 0(\bmod 4) . \operatorname{Logo}\left(2 b_{i}+a_{i}+d_{i}-3\right) \equiv 0(\bmod 4)$ ou $\left(2 b_{i}+a_{i}+d_{i}+3\right) \equiv 0(\bmod 4)$.

Se $b_{i}=0$ então $\left(a_{i}+d_{i}-3\right) \equiv 0(\bmod 4)$ ou $\left(a_{i}+d_{i}+3\right) \equiv 0$ $(\bmod 4) \Rightarrow b_{i}=0 \Rightarrow$ não pode ocorrer.

Se $b_{i}=1$ então $\left(a_{i}+d_{i}-1\right) \equiv 0(\bmod 4)$ ou $\left(a_{i}+d_{i}+5\right) \equiv 0$ $(\bmod 4) \Rightarrow\left(a_{i}, d_{i}\right) \in\{(0,-1) ;(0,1) ;(-1,0) ;(1,0)\} \Rightarrow u=d_{i}+2 b_{i}+$ $a_{i}=-\left(a_{i}+d_{i}\right) \in\{1,-1\}$ (linha 7$)$ e $u_{i, j}=-u=\left(a_{i}+d_{i}\right) \in\{1,-1\}$ (linha 12) e novo $\left(d_{i}\right)=0$ (linha 16).

Se $b_{i}=-1$ então $\left(a_{i}+d_{i}-5\right) \equiv 0(\bmod 4)$ ou $\left(a_{i}+d_{i}+1\right) \equiv 0$ $(\bmod 4) \Rightarrow\left(a_{i}, d_{i}\right) \in\{(0,1) ;(0,-1) ;(-1,0) ;(1,0)\} \Rightarrow u=d_{i}+2 b_{i}+$ $a_{i}=-\left(a_{i}+d_{i}\right) \in\{1,-1\}$ (linha 7) e $u_{i, j}=-u=\left(a_{i}+d_{i}\right) \in\{1,-1\}$ (linha 12) e novo $\left(d_{i}\right)=0$ (linha 16).

Devemos provar que $\left(4 e_{i, j+3}+2 c_{i}+b_{i}+\operatorname{novo}\left(d_{i}\right)\right) \equiv 1(\bmod 2) \Rightarrow$ $\left(b_{i}+\operatorname{novo}\left(d_{i}\right)-1\right) \equiv 0(\bmod 2)$ que se mantém para todos os valores de $b_{i}$.

\subsection{Otimalidade da FEC}

Nesta seção descreveremos a prova apresentada por Solinas de que a FEC possui peso de Hamming conjunto mínimo dentre todas as expansões binárias conjuntas com sinal. Esta prova segue a seguinte abordagem: demonstrar que o peso de Hamming conjunto da saída do Algoritmo 8.2 é menor ou igual do que da sua entrada.

Consideremos $e_{i, j}$ a entrada do Algoritmo 8.2 e $u_{i, j}$ como sua saída ( $i \in$ $\{0,1\}$ e $0 \leq j \leq m)$. Para um determinado $k$, foi definido $W_{k}$ como

$$
\begin{gathered}
\left(w_{0, m}, \ldots, w_{0,1}, w_{0,0}\right) \\
\left(w_{1, m}, \ldots, w_{1,1}, w_{1,0}\right),
\end{gathered}
$$

onde

$$
\begin{array}{r}
w_{i, j}=e_{i, j} \text { para } j \geq k \\
u_{i, j} \text { para } j<k .
\end{array}
$$


Assim sendo $W_{0}$ é a següência de entrada e $W_{m+1}$ é a seqüência de saída. O passo $j$ do Algoritmo 8.2 substitui $W_{j}$ por $W_{j+1}$.

Se $e_{0, j}$ ou $e_{1, j}$ (entrada) é diferente de zero e $u_{0, j}=u_{1, j}=0$ (saída), então o peso de Hamming conjunto de $W_{j+1}$ é o de $W_{j}$ menos 1 e portanto ocorre uma perda no passo $j$.

Similarmente, se $u_{0, j}$ ou $u_{1, j}$ (saída) é diferente de zero e $e_{0, j}=e_{1, j}=0$ (entrada), então o peso de Hamming conjunto de $W_{j+1}$ é o de $W_{j}$ mais 1 e portanto ocorre um ganho no passo $j$.

Para provar que o Algoritmo 8.2 sempre produz uma saída cujo peso de Hamming conjunto é menor ou igual do que da entrada, deve-se demonstrar que o número de perdas é no mínimo igual ao número de ganhos.

Para formalizar a prova, foi apresentado um conjunto de estados $\left(S_{j}\right)$ que permite descrever em termos de transação de estados, quando ocorre perdas ou ganhos.

Distribui-se inicialmente os 51 casos de $R_{i, j}$ nos seguintes agrupamentos

(a) $L_{i, j}$ é ímpar, $d_{i}=0$

(b) $L_{i, j}$ é ímpar, $d_{i}= \pm 1, b_{i}=0$

(c) $L_{i, j}$ é ímpar, $d_{i}= \pm 1, b_{i}= \pm 1$

(d) $L_{i, j}$ é par, $d_{i}=0$

(e) $L_{i, j} \equiv 2(\bmod 4), d_{i}= \pm 1, b_{i}=0$

(f) Outros casos em que $L_{i, j}$ é par.

Os agrupamentos podem ser combinados obtendo-se, então, um novo conjunto de estados.

\begin{tabular}{|c|l|c|}
\hline$S_{j}$ & \multicolumn{1}{|c|}{$R_{0, j}$ e $R_{1, j}$} & $S_{j+1}$ \\
\hline \hline $\begin{array}{c}L \\
\text { (perda) }\end{array}$ & $\begin{array}{l}\text { • } R_{0, j} \text { e } R_{1, j} \text { podem conter (d), (e), ou (f), } \\
\text { porém não ocorre o caso ((d),(d)) }\end{array}$ & $L, M, N$, ou $P$ \\
\hline $\begin{array}{c}M \\
\text { (ganho) }\end{array}$ & $\begin{array}{l}\bullet \quad R_{0, j} \text { e } R_{1, j} \text { podem conter (b), (c), ou (d), } \\
\text { porém não ocorre o caso ((d),(d)) }\end{array}$ & $L$ ou $N$ \\
\hline$N$ & $\begin{array}{l}\bullet \quad \text { Um deles contém (a) e o outro (a), (c), (d), } \\
\text { ou (f); ou } \\
\text { ((d),(d)) }\end{array}$ & $L$ ou $N$ \\
\hline$P$ & outros 12 casos & $L, M, N$ ou $P$ \\
\hline
\end{tabular}

Pode ser verificado que um caso englobado pelos estados $M$ e $N$ é seguido por um caso englobado pelos estados $L$ e $N$. Também pode ser verificado que o estado $L$ consiste precisamente dos casos em que existe perda de peso, 
e o estado $M$ dos casos em que existe ganho de peso. Não existe ganho ou perda nos estados $N$ e $P$.

Desde que $d_{i}$, é inicialmente 0 no Algoritmo 8.2, $R_{i, j}$ inicia dentro dos agrupamentos (a) ou (d). Portanto o primeiro estado é sempre $N$.

Desde que no passo final do algoritmo, $a_{i}, b_{i}$ e $c_{i}$ são 0 , os valores finais de $R_{i, j}$ estão nos agrupamentos (b) ou (d). Portanto o estado final é $M$ ou $N$.

Segue que o fluxo do Algoritmo 8.2 é como se segue:

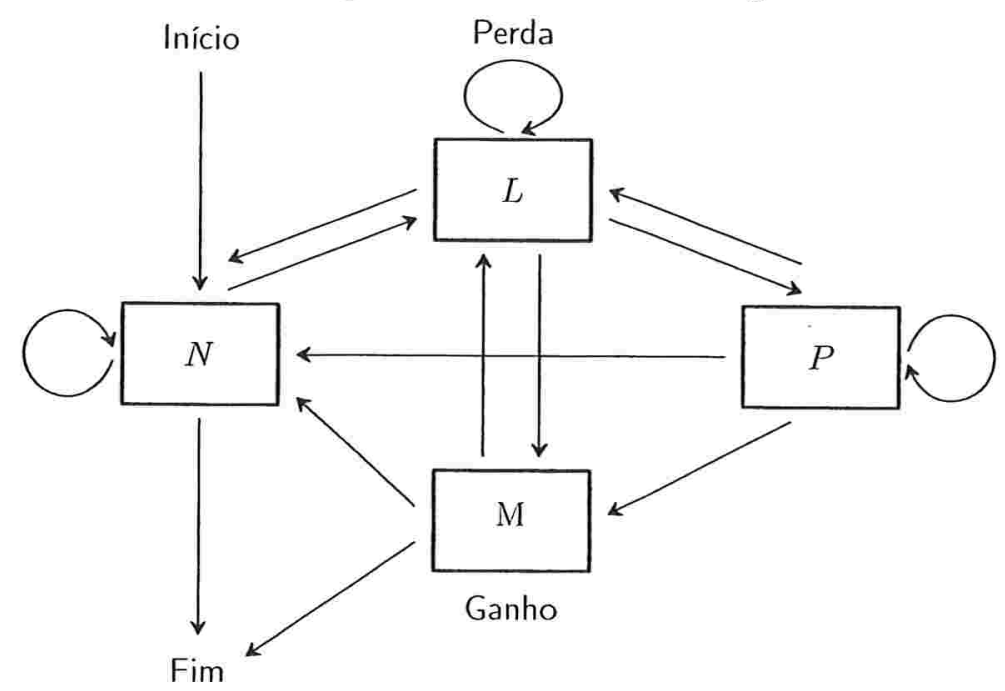

Lema 8.1. O peso de Hamming conjunto da saída do Algoritmo 8.2 não é maior do que a entrada.

Demonstração. Através do diagrama percebemos que toda visita ao estado $M$ deve ser precedida de uma visita ao estado $L$. Portanto o número total de perdas do peso é de no mínimo o número total de ganho de pesos.

Teorema 8.3. O peso de Hamming conjunto de qualquer expansão binária com sinal conjunta de inteiros positivos $K_{0}$ e $K_{1}$ é maior ou igual ao FEC de $K_{1}$ e $K_{0}$.

Demonstração. Dado qualquer expansão binária com sinal de $K$, pode-se fazer uma redução substituindo-se coeficientes adjacentes $\langle 1, \overline{1}\rangle$ por $\langle 0,1\rangle$ e $\langle\overline{1}, 1\rangle$ por $\langle 0, \overline{1}\rangle$. Por exemplo, pode-se reduzir $1 \overline{1} 00$ a 0100 e $\overline{1} 100$ a $0 \overline{1} 00$.

Desta forma, produz-se uma expansão reduzida de $K$ cujas posições diferentes 0 são um subconjunto da expansão original. Portanto qualquer expansão binária com sinal conjunta pode ser reduzida para produzir uma expansão reduzida cujo peso de Hamming conjunto é menor ou igual que a expansão original.

Aplicando o Algoritmo 8.2 para a expansão reduzida, obtém-se a FEC. Pelo Lema 8.1, o peso de Hamming conjunto da FEC não é maior do que a expansão reduzida e portanto não é maior do que a expansão original. 


\subsection{Eficiência do método rápido de Shamir usando a FEC}

Para se determinar o número de operações elípticas requeridas pelo método de Shamir, é necessário computar o comprimento e a densidade de Haming conjunto médio da FEC.

O comprimento fornecerá o número de duplicações requeridas, e a densidade o número de adições.

\subsubsection{Comprimento da FEC}

Se a expansão binária com sinal (8.3) é reduzida, então é fácil verificar que

$$
\left\lfloor\log _{2} \eta\right\rfloor \leq l \leq\left\lceil\log _{2} \eta\right\rceil
$$

onde $l$ é o comprimento da expansão binária com sinal.

Segue que a expansão binária com sinal reduzida é no máximo 1 bit maior do que a expansão binária ordinária. Com o resultado, a FEC é no máximo 1 bit maior do que a expansão binária do maior dos dois inteiros.

Teorema 8.4. A densidade de Hamming conjunto médio entre as representações Forma Esparsa Conjunta é 1/2.

Demonstração. Para cada $j \geq 0$, foi definido $\operatorname{Prob}_{j}(A)$ como a probabilidade de $S_{j}$ estar no estado $A$, considerando as entradas $K_{0}$ e $K_{1}$ de inteiros aleatórios e não-negativos menores que $2^{m}$ para algum $m \geq j+3$.

Desde que as saídas $u_{0, j}$ e $u_{1, j}$ do Algoritmo 8.2 são independentes das entradas $e_{0, k}$ e $e_{1, k}$ quando $k \geq j+3, \operatorname{Prob}_{j}(A)$ independe de $m$.

As probabilidades $\operatorname{Prob}_{j}(B)$ e $\operatorname{Prob}_{j}(C)$ são definidas analogamente.

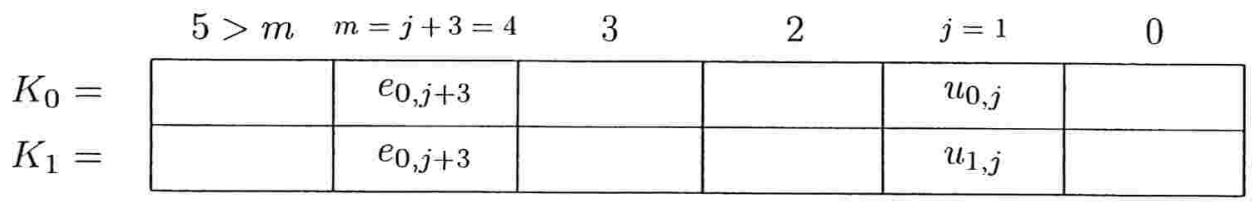

Para acomodar a restrição $m \geq j+3$ trabalha-se com a densidade de Hamining conjunta ajustada ao invés da verdadeira densidade de Hamming conjunta. A densidade de Hamming conjunta ajustada é a densidade considerando tudo menos as três posições mais à esquerda de $j$. Assintoticamente, a omissão dessas três posições não preocupa.

Desde que $u_{0, j}=u_{1, j}=0$ é uma saída somente para o estado $C$, a densidade de Hamming conjunta ajustada para entradas menores que $2^{m}$ é dada por

$$
\zeta_{m}=1-\frac{1}{m-2} \sum_{j=0}^{m-3} \operatorname{Prob}_{j}(C) .
$$


Por exemplo, para $m=5$ temos que

$$
\zeta_{5}=1-\frac{1}{3}\left(\operatorname{Prob}_{0}(C)+\operatorname{Prob}_{1}(C)+\operatorname{Prob}_{2}(C)\right) .
$$

A densidade de Hamming conjunta média é então

$$
\zeta=\lim _{m \rightarrow \infty} \zeta_{m}
$$

Para calcular $\zeta_{m}$ foi desenvolvido uma recursão.

Foi visto que se $S_{j-1}$ possui estado $A$, então $S_{j}$ possui estado $B$, e que se $S_{j-1}$ possui estado $B$, então $S_{j}$ possui estado $C$.

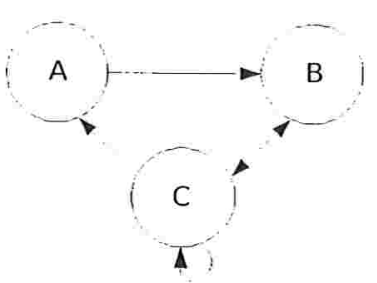

Se $S_{j-1}$ possui estado $C$, um argumento de contagem simples (ver Tabela 8.2), mostra que ${ }^{6}$ :

$$
\begin{array}{ll}
\operatorname{Prob}\left(\operatorname{estado}\left(S_{j}\right)=C\right)=1 / 4 & \operatorname{Prob}\left(\operatorname{estado}\left(S_{j}\right)=G\right)=1 / 8 \\
\operatorname{Prob}\left(\operatorname{estado}\left(S_{j}\right)=D\right)=1 / 8 & \operatorname{Prob}\left(\operatorname{estado}\left(S_{j}\right)=H\right)=1 / 16 \\
\operatorname{Prob}\left(\operatorname{estado}\left(S_{j}\right)=E\right)=1 / 16 & \operatorname{Prob}\left(\operatorname{estado}\left(S_{j}\right)=J\right)=1 / 16 \\
\operatorname{Prob}\left(\operatorname{estado}\left(S_{j}\right)=F\right)=1 / 16 & \operatorname{Prob}\left(\operatorname{estado}\left(S_{j}\right)=K\right)=1 / 4
\end{array}
$$

Tal que:

$$
\operatorname{Prob}\left(\operatorname{estado}\left(S_{j}\right)=A\right)=1 / 4 \quad \operatorname{Prob}\left(\operatorname{estado}\left(S_{j}\right)=B\right)=1 / 2
$$

Segue que

$$
\begin{aligned}
& \operatorname{Prob}_{j+1}(A)=\frac{1}{4} \operatorname{Prob}_{j}(C) \\
& \operatorname{Prob}_{j+1}(B)=\frac{1}{2}\left(\operatorname{Prob}_{j}(C)+\operatorname{Prob}_{j}(A)\right) \\
& \operatorname{Prob}_{j+1}(C)=\frac{1}{4}\left(\operatorname{Prob}_{j}(C)+\operatorname{Prob}_{j}(B)\right)
\end{aligned}
$$

A expressão para $\operatorname{Prob}_{j+1}(C)$ foi reescrita usando o fato de que

$$
\operatorname{Prob}_{j}(A)+\operatorname{Prob}_{j}(B)+\operatorname{Prob}_{j}(C)=1 .
$$

\footnotetext{
${ }^{6}$ Foi considerado, por conveniência numérica que a entrada $S_{j}=C$ tem como saída o estado $S_{j+1}=K$, e não $S_{j+1}=*$, o que nem sempre é uma realidade
} 
Desta forma obteve-se a recursão:

$$
\operatorname{Prob}_{j+1}(C)=1-\frac{3}{4} \operatorname{Prob}_{j}(C)-\frac{1}{8} \operatorname{Prob}_{j-1}(C) .^{7}
$$

Os valores iniciais para essa recursão são deduzidos de:

$$
\begin{aligned}
& \operatorname{Prob}_{0}(A)=\frac{1}{4} \\
& \operatorname{Prob}_{0}(B)=\frac{1}{2} \\
& \operatorname{Prob}_{0}(C)=\frac{1}{4} .
\end{aligned}
$$

Por exemplo:

$$
\begin{aligned}
& \operatorname{Prob}_{0}(C)=0,25 \\
& \operatorname{Prob}_{1}(C)=0,1875=0,5-0,3125 \\
& \operatorname{Prob}_{2}(C)=0,8281=0,5+0,3281 \\
& \operatorname{Prob}_{3}(C)=0,3556=0,5-0,1444 \\
& \operatorname{Prob}_{4}(C)=0,6298=0,5+0,1298 \\
& \operatorname{Prob}_{5}(C)=0,4832=0,5-0,0167 \\
& \operatorname{Prob}_{6}(C)=0,5589=0,5+0,0589
\end{aligned}
$$

Verifica-se que:

$$
\zeta_{m}=\frac{1}{2}+O\left(2^{-m}\right)
$$

tal que $\zeta=\frac{1}{2}$.

\subsection{Conclusão}

Neste capítulo foi mostrado como se obter a FEC de Solinas [ ], uma expansão binária conjunta com sinal, o que permite usar o método de rápido de Shamir para computar expressões do tipo $K_{0} P+K_{1} Q$ em curvas elípticas de maneira ótima.

Nossa contribuição foi detalhar a prova das características da FEC e a construção do respectivo algoritmo, da seguinte forma:

- Inserindo notas ao longo do capítulo.

$$
\begin{aligned}
\operatorname{Prob}_{j+1}(C) & =1-\operatorname{Prob}_{j+1}(A)-\operatorname{Prob}_{j+1}(B) \\
& =1-\frac{1}{4} \operatorname{Prob}_{j}(C)-\frac{1}{2}\left(\operatorname{Prob}_{j}(C)+\operatorname{Prob}_{j}(A)\right) \\
& =1-\frac{3}{4} \operatorname{Prob}_{j}(C)-\frac{1}{2} \operatorname{Prob}_{j}(A) \\
& =1-\frac{3}{4} \operatorname{Prob}_{j}(C)-\frac{1}{8} \operatorname{Prob}_{j-1}(C) .
\end{aligned}
$$


- Melhorando explicação do algoritmo 8.1(didático) ao mostrar que o mesmo é na verdade uma modificação do algoritmo 3.5 que computa a expansão NAF.

- Mostrando a transformação do algoritmo 8.1 no algoritmo \$.2.

- Inserindo o exemplo 8.3 que simula o funcionamento dos algoritmos 8.1 e 8.2 .

- Corrigindo um erro no sinal existente nas regras 4 e 5 de transição entre estados, ou seja, as regras estavam erroneamente escritas como:

$$
\begin{aligned}
& \text { Se }\left(\begin{array}{c}
L_{i, j} \equiv 3(\bmod 4) \\
L_{1-i, j} \equiv 2(\bmod 4)
\end{array}\right) \text { então }\left(\begin{array}{c}
L_{i, j+1} \text { ímpar } \\
-
\end{array}\right) \\
& \text { e } \operatorname{Se}\left(\begin{array}{c}
L_{i, j} \equiv 1(\bmod 4) \\
L_{1-i, j} \equiv 2(\bmod 4)
\end{array}\right) \text { então }\left(\begin{array}{c}
L_{i, j+1} \equiv 0(\bmod 4) \\
-
\end{array}\right)
\end{aligned}
$$

O correto é o seguinte:

$$
\begin{aligned}
& \text { Se }\left(\begin{array}{c}
L_{i, j} \equiv \pm 3(\bmod 4) \\
L_{1-i, j} \equiv 2(\bmod 4)
\end{array}\right) \text { então }\left(\begin{array}{c}
L_{i, j+1} \text { ímpar } \\
-
\end{array}\right) \\
& \text { e } \\
& \text { Se }\left(\begin{array}{c}
L_{i, j} \equiv \pm 1(\bmod 4) \\
L_{1-i, j} \equiv 2(\bmod 4)
\end{array}\right) \text { então }\left(\begin{array}{c}
L_{i, j+1} \equiv 0(\bmod 4) \\
-
\end{array}\right)
\end{aligned}
$$

- Provando, na seção 8.51, as regras de transição entre os estados do algoritmo 8.2.

- Melhorando a tabela 8.2 através da inserção das regras utilizadas na transição entre os estados.

- Construindo a tabela com os possíveis valores de $R_{i, j}$.

- Construindo diagramas de transição entre estados.

- Ressaltando que na prova do Teorema 8.4, o autor considerou que a entrada $S_{j}=C$ tem como saída $S_{j+1}=K$ e não $S_{j+1}=*$, o que nem sempre é uma realidade.

- Corrigindo um erro existente em um passo da prova do Teorema 84 , ou seja, no artigo está escrito:

Desta forma obteve-se a recursão:

$$
\operatorname{Prob}_{j+1}(C)=1-\frac{3}{4} \operatorname{Prob}_{j}(C)-\frac{1}{4} \operatorname{Prob}_{j-1}(C)
$$


quando o correto é

Desta forma obteve-se a recursão:

$$
\operatorname{Prob}_{j+1}(C)=1-\frac{3}{4} \operatorname{Prob}_{j}(C)-\frac{1}{8} \operatorname{Prob}_{j-1}(C)
$$




\section{Capítulo 9}

\section{Método GLV}

Curvas de Koblitz são curvas elípticas definidas sobre corpos de característica 2 e foram propostas pela primeira vez para uso criptográfico em [10]. A vantagem principal das curvas de Koblitz é que o endomorfismo de Frobenius pode ser explorado para conseguir algoritmos de multiplicação de pontos mais rápidos e sem o uso de qualquer duplicação de pontos [, 2$]$. Esta técnica pode ser generalizada para o uso em qualquer endomorfismo, particularmente para endomorfismos eficientes em curvas definidas sobre corpos primos, no entanto, geralmente é ineficiente. Smart ([]]) descreveu como aplicar o método da expansão de Frobenius em curvas definidas sobre pequenos corpos primos que é similar ao método de Müller [1], descrito no capítulo 7 . No referido artigo ([]]), Smart conclui, com base em suas simulações, que usar corpos de características ímpar é entre 10 a 100 vezes mais vagaroso do que usar um corpo de característica par da mesma ordem.

O objetivo deste capítulo é apresentar a técnica conhecida como GLV, devido a seus autores R. Gallant, R. Lambert, e S. Vanstone [1] para aumentar a velocidade da multiplicação de pontos em curvas elípticas que possuam um endomorfismo computável eficientemente. Embora a técnica não seja tão eficiente quanto o método de Solinas para curvas de Koblitz [21,2, 2], ela é útil para aumentar a velocidlade da multiplicação de pontos em uma classe mais ampla de curvas elípticas como, por exemplo, certas curvas definidas sobre corpos primos. Tais curvas elípticas foram incluídas no padrão WAP WTLS (Wireless Transport Layer Security) [

\subsection{Endomorfismos}

Seja $E$ uma curva elíptica definida sobre um corpo finito $\mathbb{F}_{q}$. Para qualquer $n \geq 1$, o grupo de pontos $\mathbb{F}_{q^{n}}$ sobre $E$ é denotado por $E_{n}$.

Um endomorfismo de $E$ é um mapeamento sobrejetivo definido por $\phi: E \rightarrow$ $E$ e satisfazendo $\phi(\mathcal{O})=\mathcal{O}$, tal que, se $P_{1}=\left(x_{1}, y_{1}\right), P_{2}=\left(x_{2}, y_{2}\right)$, onde $P_{1}, P_{2} \in E_{n}$, então $\phi\left(P_{1}+P_{2}\right)=\phi\left(P_{1}\right)+\phi\left(P_{2}\right)$ e $\phi\left(K P_{1}\right)=k \phi\left(P_{1}\right)$, onde 
$K \in \mathbb{Z}$ e $n \geq 1$.

Exemplo 9.1. Seja $E$ uma curva elíptica definida sobre $\mathbb{F}_{q}$. Para cada $K \in$ $\mathbb{Z}$ o mapeamento multiplicação por $K,[K]: E \rightarrow E$ definido por $P \mapsto K P$ é um endomorfismo definido sobre $\mathbb{F}_{q}$.

Exemplo 9.2. Seja $E$ uma curva elíptica definida sobre $\mathbb{F}_{q}$. O mapeamento $\phi: E \rightarrow E$ definido por $(x, y) \mapsto\left(x^{q}, y^{q}\right)$ e $\mathcal{O} \mapsto \mathcal{O}$ é um endomorfismo definido sobre $\mathbb{F}_{q}$, chamado endomorfismo de Frobenius. Se $q$ é uma potência de 2 e $P \in E_{n}$, então $\phi(P)$ pode ser computado eficientemente desde que a elevação ao quadrado é substancialmente mais rápida do que a multiplicação em um corpo finito de característica 2. O endomorfismo de Frobenius satisfaz a equação característica $\phi^{2}-c \phi+q=0$.

Exemplo 9.3. Seja $p \equiv 1(\bmod 4)$ um número primo, e considere a curva elíptica

$$
E_{1}: y^{2}=x^{3}+a x
$$

definida sobre $\mathbb{F}_{p}$. Seja $\alpha \in \mathbb{F}_{p}$ um elemento de ordem $4\left(\alpha^{4} \bmod p \equiv 1\right)$. O mapeamento $\phi: E_{1} \rightarrow E_{1}$ definido por $(x, y) \mapsto(-x, \alpha y)$ e $\mathcal{O} \mapsto \mathcal{O}$ é um endomorfismo definido sobre $\mathbb{F}_{p}$ que satisfaz a equação $\phi^{2}+1=0$.

Exemplo 9.4. Seja $p \equiv 1(\bmod 3)$ um número primo, e considere a curva elíptica

$$
E_{2}: y^{2}=x^{3}+b
$$

definida sobre $\mathbb{F}_{p}$. Seja $\beta \in \mathbb{F}_{p}$ um elemento de ordem $3\left(\beta^{3} \bmod p \equiv 1\right)$. $\mathrm{O}$ mapeamento $\phi: E_{2} \rightarrow E_{2}$ definido por $(x, y) \mapsto(\beta x, y)$ e $\mathcal{O} \mapsto \mathcal{O}$ é um endomorfismo definido sobre $\mathbb{F}_{p}$ que satisfaz a equação $\phi^{2}+\phi+1=0$.

Exemplo 9.5. Seja $p>3$ um primo tal que -7 é un resíduo quadrático módulo $p$, ou seja, $\exists x$ tal que $x^{2} \equiv-7 \bmod p$, e sejam $w=(1+\sqrt{-7}) / 2$ e $a=(w-3) / 4$. Considere a curva elíptica

$$
E_{3}: y^{2}=x^{3}-(3 / 4) x^{2}-2 x-1
$$

definida sobre $\mathbb{F}_{p}$. Então o mapeamento $\phi: E_{3} \rightarrow E_{3}$ definido por

$$
(x, y) \rightarrow\left(\frac{x^{2}-w}{w^{2}(x-a)}, y \frac{x^{2}-2 a x+w}{w^{3}(x-a)^{2}}\right)
$$

e $\mathcal{O} \mapsto \mathcal{O}$ é um endomorfismo definido sobre $\mathbb{F}_{p}$ que satisfaz a equação $\phi^{2}-\phi+2=0$. Computar este endomorfismo é um pouco mais difícil do que duplicar um ponto.

Exemplo 9.6. Seja $p>3$ um primo tal que -2 é um resíduo quadrático módulo $p$, ou seja, $\exists x$ tal que $x^{2} \equiv-2 \bmod p$. Considere a curva elíptica

$$
E_{4}: y^{2}=4 x^{3}-30 x-28
$$


Definida sobre $\mathbb{F}_{p}$. Então o mapeamento $\phi: E_{4} \rightarrow E_{4}$ definido por

$$
(x, y) \rightarrow\left(-\frac{2 x^{2}+4 x+9}{4(x+2)},-\frac{2 x^{2}+8 x-1}{4 \sqrt{-2}(x+2)^{2}} y\right)
$$

e $\mathcal{O} \mapsto \mathcal{O}$ é um endomorfismo definido sobre $\mathbb{F}_{p}$ que satisfaz a equação $\phi^{2}+2=0$. Computar este endomorfismo é um pouco mais difícil do que duplicar um ponto.

Os métodos para multiplicação de um ponto existentes em [11, $14,23,24]$ que exploram um endomorfismo eficiente usam o endomorfismo de Frobenius ${ }^{1}$. Seja $E$ uma curva elíptica definida sobre um pequeno corpo $\mathbb{F}_{q}$ de característica 2, e seja $\varphi$ o endomorfismo de Frobenius. Para computar $K P$, onde $P \in E_{n}$, estes métodos primeiro computam $K^{\prime} \equiv K \bmod \left(\varphi^{n}-1\right)$ no anel $Z[\varphi]$. Então, computa-se a expansão $\phi$-ádica $K^{\prime}=\sum_{i=0}^{l} k_{i} \varphi^{i}$, onde os $k_{i}$ são elementos de um pequeno conjunto $\{-q / 2, \ldots, q / 2\}$, e $l \leq n$. Finalmente, $K P$ pode ser eficientemente computado como se segue:

$$
K P=K^{\prime} P=\sum_{i=0}^{l} k_{i} \varphi^{i}(P)
$$

A vagarosa duplicação foi substituída pelo ligeiro cálculo do mapeamento de Frobenius.

Os métodos baseados na expansão do mapeamento de Frobenius podem ser a princípio ser estendidos para qualquer endomorfismo $\phi$. Contudo, essa técnicas não serão eficientes se a computação de $\phi$ for mais custosa do que a duplicação de um ponto. Além disso, pode-se não ter $\phi^{n}-1=0^{2}$, condição satisfeita pelo endomorfismo de Frobenius, tal que a expansão $\phi$ ádica de $K$ torna-se significativamente mais longa que a expansão binária de $K$. Finalmente, as técnicas existentes não se aplicam quando a norma de $\phi$ é igual a 1 (como é o caso dos exemplos 9.3 e 9.4) desde que estas técnicas requerem uma operação de divisão por $\phi$ que permita um resto tendo norma menor do que a norma de $\phi$.

Na próxima Seção 9.2 descreve o novo método que explora endomorfismos computáveis eficientemente tal como od dos exemplos 9.3, 9.4, 9.5 e 9.6 para aumentar a velocidade da multiplicação de pontos.

\subsection{A técnica empregada}

\subsubsection{Linhas gerais}

Seja $E$ um a curva elíptica definida sobre $\mathbb{F}_{q}$ e seja $\phi$ um endomorfismo computável eficientemente definido sobre $\mathbb{F}_{q}$. Seja $P \in E$ um ponto de ordem prima $\eta(\eta P \equiv \mathcal{O})$. O mapeamento $\phi$ atua em $\langle P\rangle^{3}$ como um mapeamento

\footnotetext{
${ }^{1}$ ver capítulos 5,6 , e 7

${ }^{2}$ ver explicação na parte final da demonstração do Teorema 5.1 na página 13

${ }^{3}$ Crupo constituído de todos os produtos finitos de $P$
} 
multiplicativo $[\lambda]$, onde $\lambda$ é uma raiz do polinômio característico de $\phi$ módulo $\eta$, ou seja, $\phi(Q)=\lambda Q$ para $Q \in\langle P\rangle{ }^{4}$

O problema em questão é computar $K Q$, onde $Q \in\langle P\rangle$, para um $K$ aleatoriamente selecionado do intervalo $[1, \eta-1]$. Suponha que se possa escrever de maneira eficiente $K \equiv z_{0}+z_{1} \lambda(\bmod \eta)$, onde $z_{0}, z_{1} \in[0,\lfloor\sqrt{\eta}\rfloor]$. Então temos

$$
K Q=\left(z_{0}+z_{1} \lambda\right) Q=z_{0} Q+z_{1}(\lambda Q)=z_{0} Q+z_{1} \phi(Q)
$$

Desta forma (9.4) pode ser computado usando o método da multiplicação múltipla simultânea com janela de tamanho $w$. Pode-se, alternativamente, aplicar a FEC em $z_{0}$ e $z_{1}$ antes da computação de $z_{0} P+z_{1} \phi(Q)$.

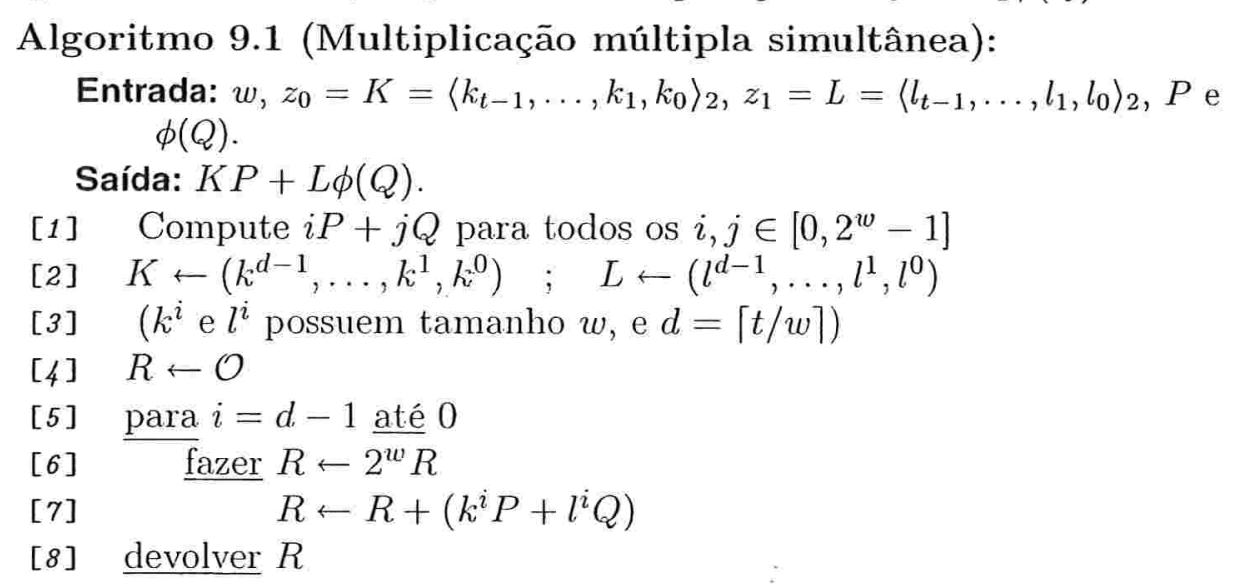

Desde que a quantidade de bits de $z_{0}$ e $z_{1}$ em (9.4) é metade da quantidade de bits de $K$, espera-se um significante aumento na velocidade, pois foi suprimido um grande número de duplicações de pontos a custa da supressão algumas adições ${ }^{5}$.

Como uma estimativa, para $l=160$ o método da multiplicação múltipla simultânea (com janela igual a 3) é 50\% mais rápido do que o método da janela deslizante com sinal ( com janela igual 4).

Exemplo 9.7. Um exemplo de curva elíptica em que o método é aplicável é $E: y^{2}=x^{3}+3$ sobre o corpo primo $\mathbb{F}_{p}$, onde

$$
p=1461501637330902918203684832716283019655932313743
$$

é um primo de 160 bits, e

$$
\# E\left(\mathbb{F}_{p}\right)=1461501637330902918203687013445034429194588307251
$$

é primo. Esta curva é incluída na especificação WAP do protocolo WTLS [2].

\footnotetext{
${ }^{4} \mathrm{O}$ valor de $\lambda$ pode ser obtido com o uso de [: ] (ver implementação no Apendice B).

${ }^{5} K \leq \eta$ e $z_{i} \leq \sqrt{\eta}$ produzem, respectivamente, $\log _{2} \eta$ e $\left(\log _{2} \eta\right) / 2$ bits
} 


\subsubsection{Decomposição de $K$}

Nesta seção descrevemos o algoritmo que tem como entrada $\eta, \lambda, K \in \mathbb{Z}$ $(K \in[1, \eta-1])$, e como saídas $z_{0}, z_{1} \in \mathbb{Z}$ tal que $K \equiv\left(z_{0}+z_{1} \lambda\right) \bmod \eta$. Os inteiros $z_{0}$ e $z_{1}$ são curtos, ou, de maneira equivalente, o vetor $\left(z_{0}, z_{1}\right) \in \mathbb{Z} \times \mathbb{Z}$ possui pequena norma Euclidiana.

Seja $G \in \mathbb{Z} \times \mathbb{Z}$ e considere o homomorfismo $f: G \rightarrow \mathbb{Z}_{n}$ (ver apêndice A) definido por $(i, j) \mapsto(i+\lambda j) \bmod \eta$. Deseja-se encontrar um vetor $u=$ $(i, j) \in \mathbb{Z}$ de pequena norma Euclidiana tal que $f(u)=K$ (ou seja, $(i, j)$ tal que $(i+\lambda j) \bmod \eta=K)$ de tal forma que os componentes de $u$ possam ser usados como os desejados $z_{0}$ e $z_{1}$. É fácil encontrar um vetor $u \in \mathbb{Z}$ tal que $f(u)=K ; u=(K, 0)$ é tal vetor. O problema em questão é encontrar um vetor $u$ de pequena norma Euclidiana.

A abordagem é a seguinte: encontrar os vetores $v_{0}, v_{1} \in \mathbb{Z}$ linearmente independentes tais que $f\left(v_{0}\right)=f\left(v_{1}\right)=0$ (primeira parte); em seguida encontrar um vetor $v$ no reticulado gerado por $v_{0}$ e $v_{1}$ que é fechado em $(K, 0)$ (segunda parte) e, desta forma obter $u=(K, 0)-v$, um vetor com pequena norma Euclidiana tal que $f(u)=f((K, 0))-f(v)=K$ (lema 9.1).

Primeira parte (encontrando $v_{0}$ e $v_{1}$ )

O problema de encontrar dois vetores $v_{0}$ e $v_{1}$ independentes de pequena norma Euclidiana tais que $f\left(v_{0}\right)=f\left(v_{1}\right)=0$ pode ser resolvido usando-se uma adaptação do Algoritmo de Euclides estendido (Algoritmo 9.2). Aplicase o algoritmo de Euclides estendido para encontrar o máximo dividor comum de $\eta$ e $\lambda$ (este mdc é 1 , desde que $\eta$ é primo).

\section{Algoritmo 9.2 (Algoritmo de Euclides estendido):}

Entrada: Dois inteiros não negativos de $\eta$ e $\lambda$ com de $\eta \geq \lambda$.

Saída: $r=\operatorname{mdc}(\eta, \lambda)$ e os inteiros $s$ e $t$ satisfazendo $s \eta+t \lambda=r$.

[1] $\underline{\text { se }} \lambda=0$

[2] $\quad$ então $r \leftarrow \eta ; s \leftarrow 1 ; t \leftarrow 0$

[3] devolver $(r, s, t)$

[4] $s_{2} \leftarrow 1 ; s_{1} \leftarrow 0 ; t_{2} \leftarrow 0 ; t_{1} \leftarrow 1$

[5] enquanto $\lambda>0$

[6] $\quad$ fazer $q \leftarrow\lfloor\eta / \lambda\rfloor ; r \leftarrow \eta-q \lambda ; s \leftarrow s_{2}-q s_{1} ; t \leftarrow t_{2}-q t_{1}$

[7] $\quad \eta \leftarrow \lambda ; \lambda \leftarrow r ; s_{2} \leftarrow s_{1} ; s_{1} \leftarrow s ; t_{2} \leftarrow t_{1} ; t_{1} \leftarrow t$

[8] $\quad r \leftarrow \eta ; s \leftarrow s_{2} ; t \leftarrow t_{2}$;

[9] devolver $(r, s, t)$

O algoritmo produz uma seqüência de equações

$$
s_{i} \eta+t_{i} \lambda=r_{i} \quad i=0,1,2, \ldots
$$

onde $\left(s_{0}=1, t_{0}=0, r_{0}=\eta\right)$ e $\left(s_{1}=0, t_{1}=1, r_{1}=\lambda\right)$ e $r_{i} \geq 0$ para todos $i$. As propriedades do algoritmo, que estão descritas no Lema 9.1 podem ser facilmente provadas por indução. 
Lema 9.1. Seja $s_{i}, t_{i}, r_{i}$ a seqüência de variáveis em (9.5) produzida pela aplicação do algoritmo de Euclides Estendido para inteiros positivos $\eta$ e $\lambda$. Então:

i) $r_{i}>r_{i+1} \geq 0$ para todos os $i \geq 0$;

j) $\left|s_{i}\right|<\left|s_{i+1}\right|$ para $i \geq 1$;

k) $\left|t_{i}\right|<\left|t_{i+1}\right|$ para $i \geq 0$;

1) $r_{i-1}\left|t_{i}\right|+r_{i}\left|t_{i-1}\right|=\eta$ para todos os $i \geq 1$.

Seja $m$ o maior índice tal que $r_{m} \geq \sqrt{\eta}$. Como $r_{m}\left|t_{m+1}\right|+r_{m+1}\left|t_{m}\right|=\eta$, então $\left|t_{m+1}\right|<\sqrt{\eta}$.

Toma-se

$$
v_{0}=\left(r_{m+1},-t_{m+1}\right) .
$$

Por (9.5) temos $f\left(v_{0}\right)=0 .{ }^{6}$ e desde que $\left|t_{m+1}\right|<\sqrt{\eta}$ e $\left|r_{m+1}\right|<\sqrt{\eta}$, temos que $\left\|v_{0}\right\|<2 \sqrt{\eta}$.

Também tomamos $v_{1}$ como o mais curto entre

$$
\left(r_{m},-t_{m}\right) \text { e }\left(r_{m+2},-t_{m+2}\right)
$$

Novamente, por (9.5), temos que $f\left(v_{1}\right)=0$. Heuristicamente, espera-se que $v_{1}$ possua pequena norma Euclidiana, mas isto não pode ser provado. ${ }^{7}$

Observa-se que $v_{0}$ e $v_{1}$ são linearmente independentes desde que de outra forma, considerando $v_{1}=\left(r_{m},-t_{m}\right)$, teríamos

$$
r_{m+1} / r_{m}=-t_{m+1} /-t_{m}=t_{m+1} / t_{m} .
$$

Entretanto $r_{m+1} / r_{m}<1$ pelo Lema 9.1 (i) e $\left|t_{m+1} / t_{m}\right|>1$ pelo Lema 9.1 (k). Note que $v_{0}$ e $v_{1}$ somente dependem de $\eta$ e $\lambda$ (e não de $K$ ).

Segunda parte (encontrando $v$ )

Um vetor $v$ no reticulado inteiro gerado por $v_{0}$ e $v_{1}$ que é fechado em $(K, 0)$ pode ser facilmente encontrado usando álgebra linear elementar. Considerando $(K, 0), v_{0}$ e $v_{1}$ como vetores em $\mathbb{Q} \times \mathbb{Q}$, pode-se escrever $(K, 0)=$ $\beta_{0} v_{0}+\beta_{1} v_{1}$, onde $\beta_{0}, \beta_{1} \in \mathbb{Q}$. Então arredondando $\beta_{0}$ e $\beta_{1}$ para os inteiros mais próximos, tem-se $b_{0}=\left\lfloor\beta_{0}\right\rceil$ e $b_{1}=\left\lfloor\beta_{1}\right\rceil$. Finalmente, toma-se $v=b_{0} v_{0}+b_{1} v_{1}$.

O Lema 9.2 prova que o vetor $u$ é de fato curto.

Lema 9.2. $O$ vetor $u=(K, 0)-v$, onde $v$ é construído como descrito acima, tem norma menor ou igual a $\max \left(\left\|v_{0}\right\|,\left\|v_{1}\right\|\right)$.

\footnotetext{
${ }^{6} f\left(v_{0}\right)=\left(r_{m+1}-\lambda t_{m+1}\right) \bmod \eta=\left(s_{m+1} \eta\right) \bmod \eta=0$

${ }^{7} \mathrm{Em}$ [ | os autores fizeram uma análise matemática mais detalhada.
} 
Demonstração.

$$
\begin{aligned}
u & =(K, 0)-v \\
& =(K, 0)-\left(b_{0} v_{0}+b_{1} v_{1}\right) \\
& =(K, 0)-\left[\left(\beta_{0} v_{0}+\beta_{1} v_{1}\right)+\left(b_{0}-\beta_{0}\right) v_{0}+\left(b_{1}-\beta_{1}\right) v_{1}\right] \\
& =\left(\beta_{0}-b_{0}\right) v_{0}+\left(\beta_{1}-b_{1}\right) v_{1},
\end{aligned}
$$

onde $(K, 0)=\beta_{0} v_{0}+\beta_{1} v_{1}$.

Finalmente, desde que $\left|\beta_{0}-b_{0}\right| \leq 1 / 2$ e $\left|\beta_{1}-b_{1}\right| \leq 1 / 2$, pela desigualdade triangular temos que

$$
\|u\| \leq 1 / 2\left\|v_{0}\right\|+1 / 2\left\|v_{1}\right\| \leq \max \left(\left\|v_{0}\right\|,\left\|v_{1}\right\|\right) .
$$

onde, como vimos anteriormente, $\left\|v_{0}\right\| \leq 2 \sqrt{\eta}$.

Exemplo 9.8. Suponhamos que: $\eta=4867, \lambda=333$ e $K=4860$. Nesse caso, $m=3$ (pois $\sqrt{\eta}=69,76$ ).

\begin{tabular}{|c|c|c|c|c|c|c|c|c|c|c|}
\hline$q$ & $r$ & $s$ & $t$ & $\eta$ & $\lambda$ & $s_{2}$ & $s_{1}$ & $t_{2}$ & $t_{1}$ & indice \\
\hline- & - & - & - & 4867 & 333 & 1 & 0 & 0 & 1 & 0 \\
14 & 205 & 1 & -14 & 333 & 205 & 0 & 1 & 1 & -14 & 1 \\
1 & 128 & -1 & 15 & 205 & 208 & 1 & -1 & -14 & 15 & 2 \\
1 & 77 & 2 & -29 & 128 & 77 & -1 & 2 & 15 & -29 & $3 \Leftarrow$ \\
1 & 51 & -3 & 44 & 77 & 51 & 2 & -3 & -29 & 44 & 4 \\
1 & 26 & 5 & -73 & 51 & 26 & -3 & 5 & 44 & -73 & 5 \\
1 & 25 & -8 & 117 & 26 & 25 & 5 & -8 & -73 & 117 & 6 \\
1 & 1 & 13 & -190 & 25 & 1 & -8 & 13 & 117 & -190 & 7 \\
25 & $\mathbf{0}$ & -333 & 4867 & 1 & 0 & $\mathbf{1 3}$ & -333 & $-\mathbf{1 9 0}$ & 4867 & 8 \\
\hline
\end{tabular}

A saída, portanto, é: $r=1, s=13$ e $t=-190$.

Encontrando $v_{0}$ e $v_{1}$ :

$$
v_{0}=(51,-44) \quad v_{1}=(26,73) .
$$

Encontrando v:

$$
(4860,0)=\beta_{0}(51,-44)+\beta_{1}(26,73),
$$

portanto

$$
\begin{array}{ll}
\beta_{0}=72,895 & \beta_{1}=43,936717 \\
b_{0}=73 & b_{1}=44 .
\end{array}
$$

Assim sendo,

$$
\begin{aligned}
& v=73 \times(51,-44)+44 \times(26,73)=(4867,0) \\
& u=(4860,0)-(4867,0)=(-7,0)
\end{aligned}
$$




\subsection{Conclusão}

Neste capítulo foi descrito o método devido a R. Gallant, R. Lambert, e S. Vanstone [1] que acelera a multiplicação de pontos em curvas elípticas definidas sobre corpos primos que possuam um endomorfismo computável eficientemente.

Nossa contribuição foi ressaltar ainda mais a utilidade do método destacando na introdução do capítulo que o uso dos métodos que fazem o uso do endomorfismo de Frobenius em curvas definidas sobre corpos primos não é eficiente.

Contribuímos também com a descrição do método da seguinte forma:

- Inserindo notas ao longo do capítulo

- Descrevendo o uso do software MAGMA [1] para obtenção da raiz do polinômio característico do endomorfismo eficiente da curva.

- Ressaltando que na computação da equação 9.4, pode se utilizar, de maneira alternativa, a FEC, descrita no capítulo \&, antes da multiplicação simultânea.

- Inserindo o algoritmo 9.2.

- Inserindo o exemplo 9.8 que simula a aplicação do método. 


\section{Capítulo 10}

\section{Métodos de Ciet, Lange, Sica e Quisquater}

Neste capítulo descrevemos o artigo [?] que mostra, primeiramente, como estender a expansão $\phi$-ádica das curvas de Koblitz [ ] para uma classe de curvas definidas sobre um corpo primo que possua um endomorfismo $\phi$ computável eficientemente obtendo nestas curvas uma eficiência similar na multiplicação. Em seguida, na mesma linha da FEC de Solinas [ ], apresenta a Forma $\phi$-Esparsa Conjunta ( $\phi$-FEC) ${ }^{1}$ que combina as vantagens da expansão $\phi$ e da FEC, o que possibilita que a computação de $K_{0} P+K_{1} Q$ possa. ser feita usando o endomorfismo $\phi$ ao invés de duplicações, produzindo uma significante aumento na velocidade desta computação quando $\phi$ é particularmente eficiente, como no caso das curvas de Koblitz.

\subsection{Extensão da expansão $\tau$-ádica das curvas de Koblitz para certas curvas definidas sobre cor- pos primos com endomorfismo eficiente}

Seja $\mathbb{F}_{q}$ um corpo finito. São de interesse os seguintes casos: $q=2^{r}$ ou $q=p>3$. Seja $E$ uma curva elíptica definida sobre $\mathbb{F}_{q}$ e $P \in E$ um ponto de ordem prima $\eta$. Em criptografia com curvas elípticas, é essencial poder computar facilmente $K P$ para qualquer $K \in[1, \eta-1]$. Alguns métodos utilizam o endomorfismo $\phi$ computável eficientemente $[, \ldots, 1,4, \cdots, \ldots]$. No caso das curvas de Koblitz [1, ], é padrão usar como $\phi$ o endomorfismo de Frobenius sobre $\mathbb{F}_{2}$ (frequentemente denotado por $\tau$ ), obtendo-se uma decomposição

$$
K P=k_{0} P+k_{1} \phi(P)+\cdots+k_{l} \phi^{l}(P)
$$

\footnotetext{
${ }^{1} \phi$-Joint Sparse Form no artigo original ( $\phi$-JSF)
} 
com $k_{i} \in\{0, \pm 1\}$, similar a decomposição binária de $K$, onde, pelo fato de que $\phi^{n}(P)=P$, se obtém $l \leq \log _{2} n$.

Em curvas definidas sobre corpos primos, usa-se um endomorfismo eficiente $\phi$ tal que seu polinômio característico $\phi^{2}+r \phi+s$ possua coeficientes pequenos: este é o método de Gallant-Lambert-Vanstone (GLV) [1, ]. A essência do método GLV é decompor $K P$ como

$$
K P=z_{0} P+z_{1} \phi(P) \quad \text { com } \max \left(\left\|z_{0}\right\|,\left\|z_{1}\right\|\right)=\mathcal{O}(\sqrt{\eta})
$$

e então computar $z_{0} P$ e $z_{1} \phi(P)$ simultaneamente .

Veremos que o uso da redução GLV possibilitará obter, nessas curvas, uma expansão na base $\phi$, como a expansão 10.1 , onde $k_{i} \in \mathfrak{R}=\{-u, \ldots, u\}$ e $u$ pequeno.

\subsubsection{Decomposição chave: expansão $\phi$}

O objetivo desta seção é descrever como se obtém uma decomposição na base $\phi$, onde $\phi$ é um endomorfismo da curva que possui $\phi^{2}+r \phi+s$ como polinômio característico. Vamos assumir que $s>1$. Dado $K \in \mathbb{Z}[\phi]$, desejase decompô-lo como

$$
K=k_{0}+k_{1} \phi+\cdots+k_{l} \phi^{l}
$$

onde $k_{i} \in \Re=\{-u, \ldots, u\}$.

Os coeficientes $k_{0}, \ldots, k_{l}$ são obtidos indutivamente.

Lema 10.1. Seja $z_{0}, z_{1} \in \mathbb{Z}$, então $\phi$ divide $z_{0}+z_{1} \phi$ em. $Z[\phi]$ se e somente se $s$ divide $z_{0}$. (Compare com o Lema \%.1.)

Isto implica que uma escolha de $\Re$ como conjunto completo de resíduos módulo $s$ é necessário e suficiente para garantir a existência e a unicidade de $k_{0}$. Como a negação de um ponto de uma curva possui custo computacional insignificante, escolhe-se um conjunto de restos simétricos a zero. Quando $s$ é ímpar, uma escolha completa é $\mathfrak{R}=\{-(s-1) / 2, \ldots,(s-1) / 2\}$. Quando $s$ é par, $\mathfrak{R}=\{-s / 2, \ldots, s / 2\}$.

Seja $z=z_{0}+z_{1} \phi \in \mathbb{Z}[\phi]$. Para decompor $z$ faz-se $k_{0} \equiv z_{0} \bmod s$ e usando-se o polinômio característico de $\phi$, isto é, $s=-r \phi-\phi^{2}=-\phi(r+\phi)$, obtém-se

$$
\begin{aligned}
z & =k_{0}+\frac{z_{0}-k_{0}}{s} s+z_{1} \phi \\
& =k_{0}+\frac{z_{0}-k_{0}}{s}\left(-r \phi-\phi^{2}\right)+z_{1} \phi \\
& =k_{0}+\phi\left[\frac{z_{0}-k_{0}}{s}(-r-\phi)+z_{1}\right] \\
& =k_{0}+\phi\left[\left(\frac{k_{0}-z_{0}}{s} r+z_{1}\right)+\frac{k_{0}-z_{0}}{s} \phi\right]
\end{aligned}
$$


Substitui-se $z$ por $\left(z-k_{0}\right) / \phi$ e encontra-se $k_{1}$. Em seguida substitui-se $z$ por $\left(z-k_{1}\right) / \phi$ e encontra-se $k_{2}$ ( e assim sucessivamente ... ). Desta forma computa-se todos os coeficientes.

A questão é mostrar que este processa termina após um finito número de passos e limitar o comprimento desta expansão.

Teorema 10.1. Seja $s>1$. Então $z=z_{0}+z_{1} \phi \in \mathbb{Z}[\phi]$ pode ser expandido como (10.3) com $l \leq\left[2 \log _{s} 2 \sqrt{z_{0}^{2}-r z_{0} z_{1}+s z_{1}^{2}}\right]+3$ exceto quando $(r, s) \in$ $\{( \pm 2,2),( \pm 3,3),( \pm 4,5),( \pm 5,7)\}$. Nesses casos tem que se permitir $k_{l-1}=$ $\lceil(s+1) / 2\rceil$. (Compare com o Teorema 7.1.)

Demonstração. A prova segue a mesma linha das correspondentes provas existentes em [1] e [1]. O fato de $\phi$ ser ou não ser o endomorfismo de Frobenius não é importante neste momento e sim o fato da norma complexa de $\phi$ ser igual a $s(N(\phi)=s)$.

Para $z=K \in \mathbb{Z}$, esse teorema fornece uma decomposição com comprimento de aproximadamente $2 \log _{s}|K| \leq 2 \log _{s} \eta$ para valores de $K$ usados em criptografia. Este resultado é duas vezes maior do que uma expansão $s$-ária. Se $\phi$ é o endomorfismo de Frobenius, este comprimento pode ser diminuído fazendo a redução de $K$ a módulo $\phi^{n}-1^{2}$ antes da expansão.

Obter uma redução similar no caso das curvas definidas sobre corpos primos é, portanto, necessário para se obter vantagem desta decomposição. Contudo, fica evidente de pesquisas anteriores (ver [2]) que de fato pode-se reduzir o multiplicador $K$ substituindo-o por $z_{0}+z_{1} \phi$, com $z_{0}, z_{1} \in \mathbb{Z}$, tal que $K P=z_{0} P+z_{1} \phi(P)$ e $\max \left(\left\|z_{0}\right\|,\left\|z_{1}\right\|\right)=O(\sqrt{\eta})$. Esta abordagem é essencialmente o método de Gallant-Lambert-Vanstone (GLV) para essas curvas [1]. Então uma aplicação direta de [1, Teorema 1] fornece o seguinte Teorema.

Teorema 10.2. Seja $P$ um ponto um grande primo de ordem $\eta$ em uma curva elíptica e $\phi$ um endomorfismo tal que $\phi^{2}+r \phi+s=0$. Então, para um arbitrário $1 \leq K \leq \eta$ o algoritmo contido no Lema 10.1, junto com a redução GLV produz a decomposição (10.1) onde $k_{i} \in \Re$ (com a exceções listadas no Teorema 10.1) e $l \leq\left\lceil 2 \log _{s} \sqrt{1+|r|+s}+\log _{s} \eta\right\rceil+3$.

O Teorema 1 de [ ] afirma que qualquer múltiplo $K$ pode ser decomposto como $K P=z_{0} P+z_{1} \phi(P)$, com $\max \left\{\left|z_{0}\right|,\left|z_{1}\right|\right\}<\sqrt{1+|r|+s} \sqrt{\eta}$. Isto

\footnotetext{
${ }^{2}$ ver explicação na parte final da demonstração do Teorema 5.1 na página 43
} 
implica que, pelo Teorema 10.1,

$$
\begin{aligned}
l & \leq\left\lceil 2 \log _{s} 2 \sqrt{z_{0}^{2}-r z_{0} z_{1}+s z_{1}^{2}}\right\rceil+3 \\
l & \leq\left[2 \log _{s} 2 \sqrt{(1+|r|+s) \eta-r(1+|r|+s) \eta+s(1+|r|+s) \eta} \mid+3\right. \\
l & \leq\left[2 \log _{s} 2 \sqrt{(1+|r|+s) \eta(1-r+s)}\right\rceil+3 \\
l & \leq\left\lceil 2 \log _{s} 2+\log _{s}(1+|r|+s)+\log _{s} \eta+\log _{s}(1-r+s)\right\rceil+3 .
\end{aligned}
$$

Para propósitos práticos obtemos uma decomposição de comprimento $\log _{s} \eta$.

Esquematicamente temos:

$$
\begin{gathered}
K \\
\Downarrow \\
\hline \text { Redução GLV } \\
\Downarrow \\
K=z_{0}+z_{1} \phi \\
\Downarrow \\
\hline \begin{array}{c}
\phi \text {-ádica de } z_{0}+z_{1} \phi \\
\text { (ver seção } 10.1 .2)
\end{array} \\
\qquad \\
\hline \text { Computar KP }
\end{gathered}
$$

Quando $\phi$ é o endomorfismo de Frobenius, a expansão 10.1 conduz a um grande aumento da velocidade.

Consideraremos dois exemplos (10.1 e 10.2) publicados em [1, de endomorfismos eficientes em curvas definidas sobre corpos primos. Nestes exemplos $s=2$. Para valores maiores de $s$ a expansão fica mais curta, contudo, os casos em que $s=2$ são de interesse especial. Serão apresentados nestes exemplos a comparação entre o número de operações para computar o endomorfismo daqueles necessários para a duplicação de pontos no mesmo conjunto de coordenadas.

Exemplo 10.1 (Exemplo 9.5 do capítulo 9). Seja $p>3$ um número primo tal que -7 é um resíduo quadrático módulo $p$, ou seja, $\exists x$ tal que $x^{2} \equiv-7(\bmod p)$. Defina uma curva elíptica $E_{3}$ sobre $\mathbb{F}_{p}$ por $y^{2}=x^{3}$ $(3 / 4) x^{2}-2 x-1$. Se $w=(1+\sqrt{-7}) / 2$ e $a=(w-3) / 4$, então o mapeamento $\phi$ definido no plano afim por

$$
\phi(x, y)=\left(\frac{x^{2}-w}{w^{2}(x-a)}, \frac{y\left(x^{2}-2 a x+w\right)}{w^{3}(x-a)^{2}}\right)
$$


é um endomorfismo de $E_{3}$ definido sobre $\mathbb{F}_{p}$ com $\mathbb{Z}[\phi]=\mathbb{Z}[(1+\sqrt{-7}) / 2]$. Além disso, $\phi$ satisfaz a equação $\phi^{2}-\phi+2=0$. Em coordenadas afins, as fórmulas fornecidas anteriormente são claramente mais custosas, como já observado em [1], do que a duplicação. Contudo em coordenadas projetivas $\phi(X, Y, Z)$ é dado por

$$
\phi(X, Y, Z)=\left(E F, Y(A-2 X D+C), F^{2} B\right)
$$

com $A=X^{2}, B=w Z, C=B Z, D=a Z, E=A-C$ e $F=(X-D) w$.

Então, dado um ponto $P=(X, Y, Z)$, sua imagem por $\phi$ é computado com 8 multiplicações e 2 elevações ao quadrado comparado as 7 multiplicações e 5 elevações ao quadrado para a duplicações de pontos.

Note que a troca admissível de variáveis $(x, y) \rightarrow(x+1 / 4, y)$ transforma a equação $y^{2}=x^{3}-(3 / 4) x^{2}-2 x-1$ na equação $y^{2}=x^{3}-\frac{35}{16} x^{2}-\frac{49}{32}$ (ver seção 2.4). Esta transformação é necessária para se utilizar o software MAGMA [1] na obtenção dos parâmetros da curva.

Exemplo 10.2 (Exemplo 9.6 do Capítulo 9). Seja $p>3$ um número primo tal que -2 é o resíduo quadrático módulo $p$, ou seja, $\exists x$ tal que $x^{2} \equiv$ $-2 \bmod p$. Defina uma curva elíptica $E_{4}$ sobre $\mathbb{F}_{p}$ por $y^{2}=4 x^{3}-30 x-28$. O mapeamento $\phi$ é definido no plano afim por

$$
\phi(x, y)=\left(-\frac{2 x^{2}+4 x+9}{4(x+2)},-\frac{2 x^{2}+8 x-1}{4 \sqrt{-2}(x+2)^{2}} y\right)
$$

é um endomorfismo de $E_{4}$ definido sobre $\mathbb{F}_{p}$ com $\mathbb{Z}[\phi]=\mathbb{Z}[\sqrt{-2}]$. Além disso, $\phi$ satisfaz a equação $\phi^{2}+2=0$. Como no exemplo anterior, a fórmulas endomórficas são mais custosas do que as para duplicação em coordenadas afins. Contudo, em coordenadas projetivas o endomorfismo pode ser computado como

$$
\phi(X, Y, Z)=\left(D\left(2 A+4 B+9 Z^{2}\right),\left(2 A+8 B-Z^{2}\right) Y,-4 D C Z\right)
$$

$\operatorname{com} A=X^{2}, B=X Z, C=X+2 Z$ e $D=\sqrt{-2} C$.

Portanto, este endomorfismo é significantemente mais rápido do que a duplicação desde que dado um ponto $P$ em coordenadas projetivas, $\phi(P)$ pode ser computado com somente 6 multiplicações e 2 elevações ao quadrado.

Note que se multiplicarmos a equação $y^{2}=4 x^{3}-30 x-28$ por $4 \mathrm{e}$ fizermos a transformação de variáveis $(4 x, 4 y) \rightarrow(x, y)$, a equação torna-se $y^{2}=x^{3}-120 x-448$. Esta transformação é necessária para se utilizar o software MAGMA [!] na obtenção dos parâmetros da curva.

Uma importante constatação é a de que nem todos os valores de $p$, obtidos conforme explicitado nos exemplos acima descritos (10.1 e 10.2), são úteis para aplicações criptográficas, pois na maioria das vezes é necessário fazer a aplicação do endomorfismo mais de uma vez, ou seja, temos que $\phi^{u}(P)=\lambda P$ $(u \geq 1)$ e não $\phi(P)=\lambda P$ como era de se esperar. No Apendice B mostramos um desses casos. 


\subsubsection{Expansão $\phi$-ádica de densidade $1 / 3$}

Nesta seção será descrito como se obteve uma expansão $\phi$-ádica com densidade $1 / 3$ nas curvas dos exemplos citados anteriormente.

Em [2] Solinas considerou expansões tendo como base o endomorfismo de Frobenius $\varphi$ para curvas de Koblitz definidas sobre $\mathbb{F}_{2}$. O polinômio característico de $\varphi$ para as curvas $y+x y=x^{3}+a x^{2}+1$ definidas sobre $\mathbb{F}_{2}$ é dado por $\varphi^{2}-(-1)^{1-a} \varphi+2$. Ele introduziu uma forma não adjacente $\varphi(\varphi$-NAF) (Algoritmo 6.3) e apresentou um algoritmo para computar $K P$ como $K P=\sum_{i} k_{i} \varphi^{i}(P)$ com $k_{i} \in\{0, \pm 1\}$ e $k_{i} k_{i+1}=0$ (Algoritmo (i.4). Tal expansão possui densidade média de $1 / 3$.

Como o polinômio característico do Exemplo 10.1 coincide com o acima descrito podemos computar uma expansão $\phi$-NAF de densidade $1 / 3$ através do Algoritmo 6.4. Note que $\max \left\{z_{0}, z_{1}\right\}<\sqrt{\eta}$ e portanto o comprimento da expansão é $\log _{2} \eta$ (ver seção 6.2 ).

No exemplo 10.2 o polinômio característico é $\phi$ é $\phi^{2}+2=0$ e para se obter a densidade $1 / 3$ é necessário considerar que

$$
(x+y \phi) / \phi=y-(x / 2) \phi
$$

e que $x+y \phi$ é divisível por $\phi^{2}$ se e somente se $x$ e $y$ forem divisíveis por 2 .

Assim sendo, para a entrada $z_{0}+z_{1} \phi$ deve-se fazer $k_{0}=0$ quando $z_{0}$ for par; caso contrário, fazer $k_{0} \equiv z_{0}$ mods 4 . Isto implica que $2 \mid\left(\left(z_{0}-k_{0}\right) / 2\right)$, o que torna o próximo coeficiente igual a zero; e portanto, há no mínimo um coeficiente zero para cada coeficiente não-zero $\left(k_{i} k_{i+2}=0\right)$, conduzindo a uma densidade de $1 / 3$.

Algoritmo 10.1 ( $\phi$-ádica):

Entrada: $z_{0}, z_{1}$.

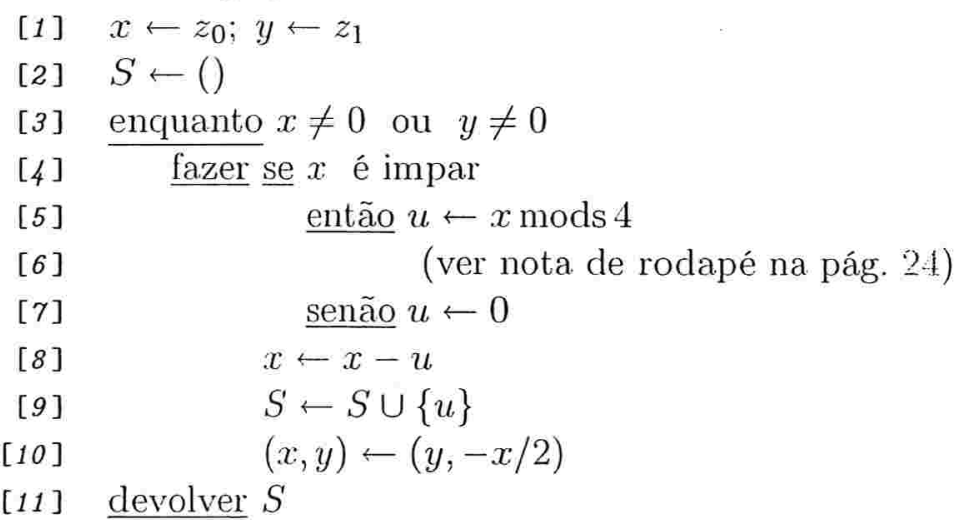

Embora o algoritmo 10.1 reduza a densidade para $1 / 3$, não produz uma expansão $\phi-\mathrm{NAF}$, ou seja, não garante que $k_{i} k_{i+1}=0$ e portanto não pode ser utilizado em métodos de multiplicação que fazem uso da $\phi$-FEC que será descrita na seção 10.2. De antemão adiantamos que isso deve-se a forma de construção do algoritmo 10.3 que computa a $\phi$-FEC. 
Para se obter uma expansão $\phi$-NAF de $z=z_{0}+z_{1} \phi$ com densidade $1 / 3$ deve-se observar que $\phi=\sqrt{-2}$ e portanto tal expansão pode ser obtida a partir da expansão NAF de $z_{0}$ e $z_{1}$. Por exemplo, se $z=11+11 \phi$, então como $\mathrm{NAF}(11)=\langle 10 \overline{1} 0 \overline{1}\rangle_{2}$ temos que $\phi-\mathrm{NAF}(11)=\langle 1000 \overline{1} 000 \overline{1}\rangle_{\phi}$. Assim sendo, $\phi-\operatorname{NAF}(z)=\langle 1000 \overline{1} 000 \overline{1}\rangle_{\phi}+\phi\langle 1000 \overline{1} 000 \overline{1}\rangle_{\phi}$.

\subsubsection{Comparação com o método NAF binário}

Nos exemplos supracitados, computar $K P$ usando expansões na base $\phi$ custa aproximadamente $l$ aplicações de $\phi$ e $l / 3$ adições, onde $l \leq m \approx \log _{2} \eta$. Essa abordagem é mais eficiente do que o método NAF binário, uma vez que computar $\phi(P)$ é menos custoso do que uma duplicação. Este resultado se mantém se o método NAF binário usa coordenadas Jacobianas.

\subsubsection{Comparação com o método GLV}

O método GLV conduz a uma decomposição $K=z_{0}+z_{1} \phi$, onde o comprimento binário de $z_{i}$ é $l / 2, \operatorname{com} l \leq m \approx \log _{2} \eta$. Considerando os $z_{i}$ na forma NAF, este método custa $l / 2$ duplicações e $l / 3$ adições $(\phi(P)$ é précomputado). O novo método que foi descrito para as curvas dos exemplos 10.1 e 10.2 trabalha com ou sem pré-computações e custa $l$ aplicações de $\phi$ e $l / 3$ adições, mas, infelizmente, nestas curvas, duas aplicações de $\phi$ é mais custoso do que uma duplicação e portanto o método GLV é melhor.

\begin{tabular}{|c|c|c|}
\hline NAF Binário & $\begin{array}{c}\text { Extensão da expansão } \\
\phi \text {-ádica das curvas de } \\
\text { Koblitz para curvas dos } \\
\text { exemplos } 10.1 \text { e } 10.2\end{array}$ & GLV c/ NAF \\
\hline$(m) D$ e $(m / 3) A$ & $(m) \phi$ e $(m / 3) A$ & $\begin{array}{c}(m / 2) D \text { e }(m / 3) A \\
(\phi(P) \text { é pré-computado })\end{array}$ \\
\hline
\end{tabular}

\subsection{Forma $\phi$-Esparsa Conjunta}

Essa seção tem como objetivo descrever a combinação da expansão $\phi$ com a FEC.

Se necessitamos computar $K_{0} P+K_{1} Q$ em um curva definida sobre corpo primo com endomorfismo eficiente, poderíamos pensar em decompor $K_{0}$ e $K_{1}$ na base $\phi$ e aplicar a FEC. No entanto, a FEC só é obtida a partir de expansões binárias.

Por outro lado, se considerarmos $K_{0}$ e $K_{1}$ na forma $\phi$-NAF, a computação de $K_{0} P+K_{1} Q$ tem um custo de $l$ aplicações de $\phi$ e $2 l / 3$ adições sem pré-computações (método simples de Shamir), onde $l \leq m \approx \log _{2} \eta$. 
Usando-se dois valores pré-computados $(P \pm Q)$ o número de adições reduz a $5 l / 9$ (método rápido de Shamir).

No mesmo espírito do trabalho de Solinas [22] os autores proporam a $\phi$ FEC, que permite a aplicação do método rápido de Shamir para as expansões $\phi$-ádica dos escalares $K_{0}$ e $K_{1}$.

Deste ponto do texto em diante, até a seção $10.5, \phi$ denotará, para efeito didático, qualquer endomorfismo tendo $\phi^{2}-\epsilon \phi+2$ como polinômio característico, com $\epsilon= \pm 1$ (o endomorfismo de Frobenius em curvas de Koblitz ou $\phi$ do exemplo 10.1).

A aplicação da $\phi$-FEC na curva do exemplo 10.2, que possui polinômio característico diferente $\left(\phi^{2}-2\right)$ será tratada na seção 10.6.

A translação da FEC de Solinas é a seguinte:

Definição 10.1 (Forma $\phi$-Esparsa Conjunta). Uma expansão com coeficientes no conjunto $\{0, \pm 1\}$ está na $\phi$-FEC se satisfaz as seguintes propriedades:

$\phi$-FEC 1 Entre três colunas consecutivas no mínimo uma é um zero duplo.

$\phi$-FEC 2 Nunca ocorre o caso em que $u_{i, j+1} u_{i, j}=\varepsilon$.

$\phi$-FEC 3 Se $u_{i, j+1} u_{i, j} \neq 0$ então $u_{i, j+1} u_{i, j}= \pm 1$ e $u_{1-i, j}=0$

$\mathrm{Na} \phi$-FEC 2 , ao invés de $u_{i, j+1} u_{i, j}=\varepsilon$, poderiamos considerar $u_{i, j+1} u_{i, j}=$ 1 ou $u_{i, j+1} u_{i, j}=-1$ e produziríamos uma $\phi$-FEC. O que corrobora esta assertiva é o fato de que a $\phi$-FEC poder também ser aplicada na curva do exemplo 10.2, cujo endomorfismo satisfaz a equação $\phi^{2}+2=0$.

Exemplo 10.3. Na esquerda damos a expansão $\phi$-NAF conjunta de $K_{0}$ e $K_{1}$ e no lado direito a $\phi$-FEC $(\varepsilon=1)$.

\begin{tabular}{ccc}
\multicolumn{1}{c}{$\phi-\mathrm{NAF}$} & \multicolumn{1}{c}{$\phi$-FEC } \\
\cline { 2 - 3 }$K_{0}$ & $\langle\overline{1} 0 \overline{1} 0 \overline{1} 0101\rangle$ & $\langle\overline{1} 00 \overline{1} 1001 \overline{1}\rangle$ \\
\cline { 2 - 3 }$K_{1}$ & $\langle 0 \overline{1} 0 \overline{1} 00010\rangle$ & $\langle 0 \overline{1} 0 \overline{1} 00010\rangle$ \\
\cline { 2 - 3 } & &
\end{tabular}

A $\phi$-FEC satisfaz propriedades análogas às propriedades da FEC.

Teorema 10.3. A $\phi-F E C$ de qualquer dois elementos $K_{0}$ e $K_{1}$ de $Z[\phi]$ existe e é única. Se a expansão $\phi-N A F$ de $K_{0}$ e $K_{1}$ tem comprimento máximo l então o algoritmo SOMA e APLICA $\phi$ SIMULTANEAMENTE computa $K_{0} P+$ $K_{1} Q$ da $\phi$-FEC com em média $(l+3)$ aplicações de $\phi$ e $(l+3) / 2$ adições de $\pm P, \pm Q, \pm(P+Q), \pm(P-Q)$.

Demonstração. A prova é similar a prova de Solinas [2]].

A minimalidade de FEC não é levada para a $\phi$-FEC, desde que $\phi$-FEC de 


\begin{tabular}{|c|c|c|}
\hline 1 & 0 & -1 \\
\hline 0 & $\varepsilon$ & 0 \\
\hline
\end{tabular}

é

\begin{tabular}{|c|c|c|c|c|c|}
\hline$-\varepsilon$ & 0 & $-\varepsilon$ & 0 & $-\varepsilon$ & 1 \\
\hline 0 & 0 & 0 & 0 & $\varepsilon$ & 0 \\
\hline
\end{tabular}

Contudo para um $l$ suficientemente grande, a $\phi$-FEC possui peso de Hamming conjunto diferindo do peso de Hamming mínimo de no máximo uma pequena constante.

O algoritmo 10.3 computa a $\phi$-FEC de dois inteiros positivos $K_{0}$ e $K_{1}$ em alguma expansão na base $\phi$.

De maneira diferente do autor, apresentamos primeiramente o algoritmo 10.2 (didático) que computa a $\phi$-FEC tendo como entrada $K_{i}=K_{i, 0}+$ $K_{i, 1} \phi$, onde $\left\langle K_{i, 0}, K_{i, 1}\right\rangle \in \mathbb{Z}^{+}$e $i \in\{0,1\}$. Fazemos isto para facilitar a compreensão do algoritmo 10.3 que possui como entrada uma expansão $\phi$ ádica de $K_{i}$, onde $i \in\{0,1\}$. No algoritmo $10.2 d_{i}=d_{i, 0}+d_{i, 1} \phi$, onde $i \in\{0,1\}$. 


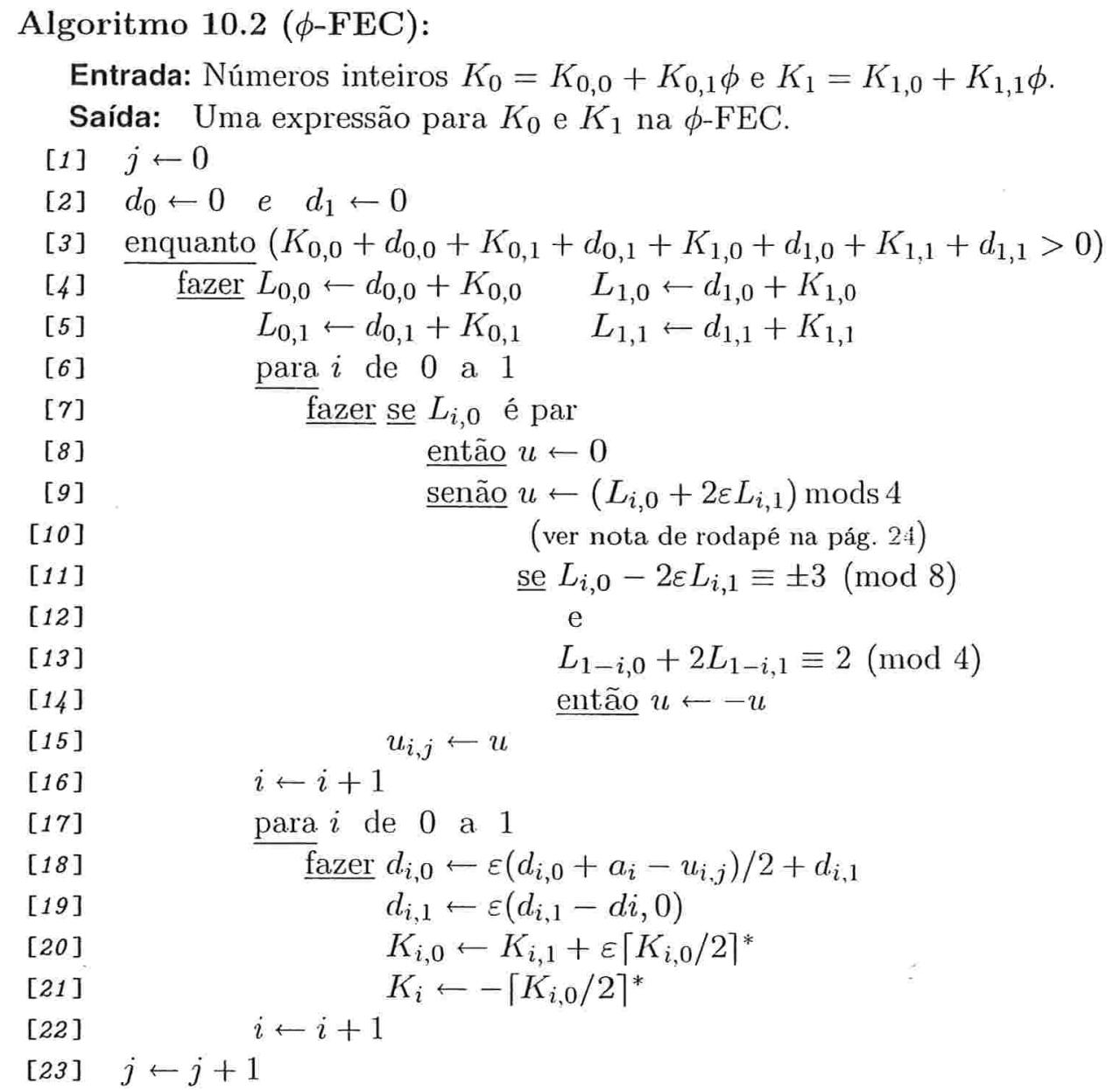

Este algoritmo ${ }^{3}$ contém a lógica do algoritmo 6.3, que computa a expansão $\phi$-NAF de $x+y \phi$. A diferença existente é a de que o algoritmo 10.2 possui a linhas adicionais (11 a 14) e a mesma operação é executada duas vezes.

Observando por este ângulo fica fácil perceber que as variáveis $L_{i, 0}=$ $K_{i, 0}+d_{i, 0}$ e $L_{i, 1}=K_{i, 1}+d_{i, 1}$ do algoritmo 10.2 correspondem, respectivamente, as variável $x$ e $y$ do algoritmo 6.3 , bem como notar que as linhas (18 a 21) efetuam na verdade a operação ${ }^{4}$

$$
\begin{gathered}
L_{i} \leftarrow\left(L_{i}-u_{i, j}\right) / \phi=\left(K_{i}+d_{i}-u_{i, j}\right) / \phi= \\
=\left\lfloor\left(K_{i, 0}+K_{i, 1} \phi\right) / \phi\right\rfloor+\left(d_{i, 0}+a_{i}-u_{i, j}+d_{i, 1} \phi\right) / \phi= \\
=\left(K_{i, 1}+\varepsilon\left\lceil K_{i, 0} / 2\right\rceil^{*}-\left\lceil K_{i, 0} / 2\right\rceil^{*} \phi\right)+\ldots
\end{gathered}
$$

\footnotetext{
${ }^{3}$ Neste algoritmo $\lceil x / 2\rceil^{*}=\lceil x / 2\rceil$ se $x \geq 0$ e $\lceil x / 2\rceil^{*}=-\lceil|x / 2|\rceil$ se $x<0$.

${ }^{4}$ Lembre-se que para $x$ par: $(x+y \phi) / \phi=(y+\varepsilon x / 2)-(x / 2) \phi$
} 


$$
\begin{gathered}
\cdots+\left(d_{i, 1}+\varepsilon\left(d_{i, 0}+a_{i}-u_{i, j}\right) / 2-\left(d_{i, 0}+a_{i}-u_{i, j}\right) \phi / 2\right)= \\
=\left(K_{i, 1}+\varepsilon\left\lceil K_{i, 0} / 2\right\rceil^{*}-\left\lceil K_{i, 0} / 2\right\rceil^{*} \phi\right)+\ldots \\
=\cdots+\left(d_{i, 1}+\varepsilon\left(d_{i, 0}+a_{i}-u_{i, j}\right) / 2+\varepsilon\left(d_{i, 1}-d_{i, 0}\right) \phi\right)
\end{gathered}
$$

onde $a_{i}$ é o valor do bit menos significativo da NAF $\phi$-ádica de $K_{i}{ }^{5}$

Vamos supor que não existissem as linhas (11 a 14). Assim sendo, em cada passo deste algoritmo em que $L_{i, 0}$ fosse ímpar, $L_{i}-u_{i, j}$ seria divisível por $\phi^{2}$ e portanto $L_{i} \leftarrow\left(L_{i}-u_{i j}\right) / \phi$, linhas (18 a 21$)$, divisível por $\phi$. Em outras palavras estaríamos computando a expansão $\phi$-NAF de $L_{i, 0}+L_{i, 1} \phi$. Note que $d_{i, 0} \leftarrow \varepsilon\left(d_{i, 0}+a_{i}-u_{i, j}\right) / 2$ e $d_{i, 0} \leftarrow \varepsilon\left(d_{i, 1}-d_{i, 0}\right)$ sempre serão 0 , pois sempre ocorrerá a igualdade $a_{i}=u_{i, j}$.

Concluímos, portanto que $d_{i}=d_{i, 0}+d_{i, 1} \phi$ armazena a mudança que poderá ocorrer no valor de $u_{i, j}$ quando as linhas (11 a 14) são inseridas. Reiteramos que o $u_{i, j}$ que seria obtido, caso não existissem as linhas (11 a 14) é aquela advinda da computação da Expansão $\phi$-NAF da entrada.

Porque as condições contidas nas linhas (11 a 14) produzem a $\phi$-FEC de $K_{0}$ e $K_{1}$ ?

As linhas (11 a 14) decorrem das translações das condições $\bmod 4 \mathrm{e}$ mod 8 contidas na linha (10) do algoritmo 8.1 que computa a FEC, ou seja,

$$
L_{i} \equiv \pm 3 \quad \bmod 8 \quad \text { e } \quad L_{i} \equiv \pm 2 \bmod 4
$$

correspondem respectivamente a

$$
L_{i} \equiv \pm(\phi-\varepsilon) \quad \bmod \phi^{3} \text { e } L_{i} \equiv \phi \bmod \phi^{2}
$$

As translações são corretas, pois as expansões binárias dos decimais 2 e $3,\langle 10\rangle_{2}$ e $\langle 11\rangle_{2}$, correspondem, respectivamente, as expansões $\langle\phi\rangle$ e $\langle\phi-\varepsilon\rangle$ na base $\phi$ (ver $\phi$-FEC 2 ).

Como $x+y \phi$ é divisível por $\phi^{3}$ se somente se $(x-2 \varepsilon y) \equiv 0 \bmod 8 \quad 6$

$$
\begin{gathered}
L_{i, 0}+L_{i, 1} \phi \equiv \pm(\phi-\varepsilon) \bmod \phi^{3} \\
\left(L_{i, 0} \pm \varepsilon\right)+\left(L_{i, 1} \mp 1\right) \phi \equiv 0 \bmod \phi^{3} \\
\left(L_{i, 0} \pm \varepsilon\right)-2 \varepsilon\left(L_{i, 1} \mp 1\right) \equiv 0 \bmod 8
\end{gathered}
$$

e portanto

$$
L_{i, 0}-2 \varepsilon L_{i, 1} \equiv \pm(3 \varepsilon) \equiv \pm 3 \quad \bmod 8
$$

\footnotetext{
${ }^{5} a_{i}=K_{i, 0}+2 \varepsilon K_{i, 1} \operatorname{mods} 4$ (se $K_{i, 0}$ ímpar) ou $a_{i}=0$ (se $K_{i, 0}$ é par).

${ }^{6} \mathrm{~A}$ demonstração é similar a contida na página
} 
Como $x+y \phi$ é divisível por $\phi^{2}$ se somente se $(x-2 \varepsilon y) \equiv 0 \bmod 4 \quad 7$

$$
\begin{gathered}
L_{i, 0}+L_{i, 1} \phi \equiv \phi \quad \bmod \phi^{2} \\
L_{i, 0}+\left(L_{i, 1}-1\right) \phi \equiv 0 \quad \bmod \phi^{2} \\
L_{i, 0}-2 \varepsilon\left(L_{i, 1}-1\right) \equiv 0 \quad \bmod 4
\end{gathered}
$$

e portanto

$$
L_{i, 0}-2 \varepsilon L_{i, 1} \equiv-2 \varepsilon \quad \bmod 4 \quad \rightarrow \quad L_{i, 0}+2 L_{i, 1} \equiv 2 \quad \bmod 4
$$

A linha (9) do algoritmo vem do fato de que se $(x-2 \varepsilon y) \equiv 0 \bmod 4$, então $(x+2 \varepsilon y) \equiv 0 \bmod 4$

O exemplo 10.4 simula a aplicação do algoritmo 10.2 se considerarmos como entrada os pares $K_{0}=3+(0) \phi$ e $K_{1}=2+(0) \phi$.

Exemplo 10.4.

\begin{tabular}{|c|c|}
\hline$K_{i, 0}$ & $K_{i, 1}$ \\
\hline \hline 3 & 0 \\
\hline 2 & 0 \\
\hline
\end{tabular}

\begin{tabular}{|c|c|c|c|c|c|}
\hline 1 & 0 & 0 & 1 & 0 & -1 \\
\hline 0 & 0 & -1 & 0 & -1 & 0 \\
\hline
\end{tabular}
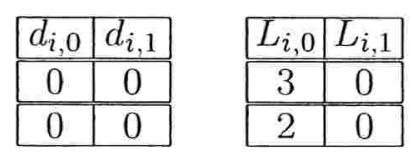

\begin{tabular}{|l|l|}
\hline 2 & -2 \\
\hline \hline 1 & -1 \\
\hline
\end{tabular}

\begin{tabular}{|c|c|c|c|c|c|}
\hline 1 & 0 & 0 & 1 & 0 & 1 \\
\hline 0 & 0 & -1 & 0 & -1 & 0 \\
\hline
\end{tabular}

\begin{tabular}{|c|c|}
\hline-1 & 1 \\
\hline \hline 0 & 0 \\
\hline
\end{tabular}

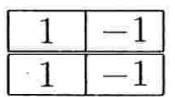

\begin{tabular}{|c|c|}
\hline-1 & -1 \\
\hline \hline 0 & -1 \\
\hline
\end{tabular}

\begin{tabular}{|l|c|c|c|c|c|}
\hline 1 & 0 & 0 & 1 & -1 & 1 \\
\hline 0 & 0 & -1 & 0 & -1 & 0 \\
\hline
\end{tabular}

\begin{tabular}{|l|l|}
\hline 1 & 0 \\
\hline \hline 0 & 0 \\
\hline
\end{tabular}

\begin{tabular}{|l|l|}
\hline 0 & -1 \\
\hline \hline 0 & -1 \\
\hline
\end{tabular}

\begin{tabular}{|l|l|}
\hline-2 & 1 \\
\hline \hline-1 & 0 \\
\hline
\end{tabular}

\begin{tabular}{|c|c|c|c|c|c|}
\hline 1 & 0 & 0 & 0 & -1 & 1 \\
\hline 0 & 0 & -1 & 0 & -1 & 0 \\
\hline
\end{tabular}

\begin{tabular}{|c|c|}
\hline 1 & -1 \\
\hline \hline 0 & 0 \\
\hline
\end{tabular}

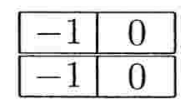

\begin{tabular}{|l|l|}
\hline 0 & 1 \\
\hline \hline 0 & 0 \\
\hline
\end{tabular}

\begin{tabular}{|l|l|l|l|l|l|}
\hline 1 & 0 & -1 & 0 & -1 & 1 \\
\hline \hline 0 & 0 & -1 & 0 & -1 & 0 \\
\hline
\end{tabular}

\begin{tabular}{|c|c|}
\hline 0 & -1 \\
\hline \hline 0 & 0 \\
\hline
\end{tabular}

\begin{tabular}{|l|l|}
\hline 0 & 0 \\
\hline 0 & 0 \\
\hline
\end{tabular}

\begin{tabular}{|l|l|}
\hline 1 & 0 \\
\hline \hline 0 & 0 \\
\hline
\end{tabular}

\begin{tabular}{|c|c|c|c|c|c|}
\hline 1 & 0 & -1 & 0 & -1 & 1 \\
\hline \hline 0 & 0 & -1 & 0 & -1 & 0 \\
\hline
\end{tabular}

\begin{tabular}{|c|c|}
\hline-1 & 0 \\
\hline \hline 0 & 0 \\
\hline
\end{tabular}

\begin{tabular}{|l|l|}
\hline 0 & 0 \\
\hline 0 & 0 \\
\hline
\end{tabular}

\begin{tabular}{|l|l|}
\hline 0 & 0 \\
\hline 0 & 0 \\
\hline
\end{tabular}

\begin{tabular}{|l|l|l|l|l|l|}
\hline 0 & 0 & -1 & 0 & -1 & 1 \\
\hline 0 & 0 & -1 & 0 & -1 & 0 \\
\hline
\end{tabular}

\begin{tabular}{|l|l|}
\hline 0 & 0 \\
\hline \hline 0 & 0 \\
\hline
\end{tabular}

\begin{tabular}{|l|l|}
\hline 0 & 0 \\
\hline \hline 0 & 0 \\
\hline
\end{tabular}

Simulação dos algoritmos 10.2 e 10.3

A partir deste ponto passamos a mostrar a conversão do algoritmo 10.2 no algoritmo 10.3

\footnotetext{
${ }^{7}$ Ver demonstração na página 50
} 


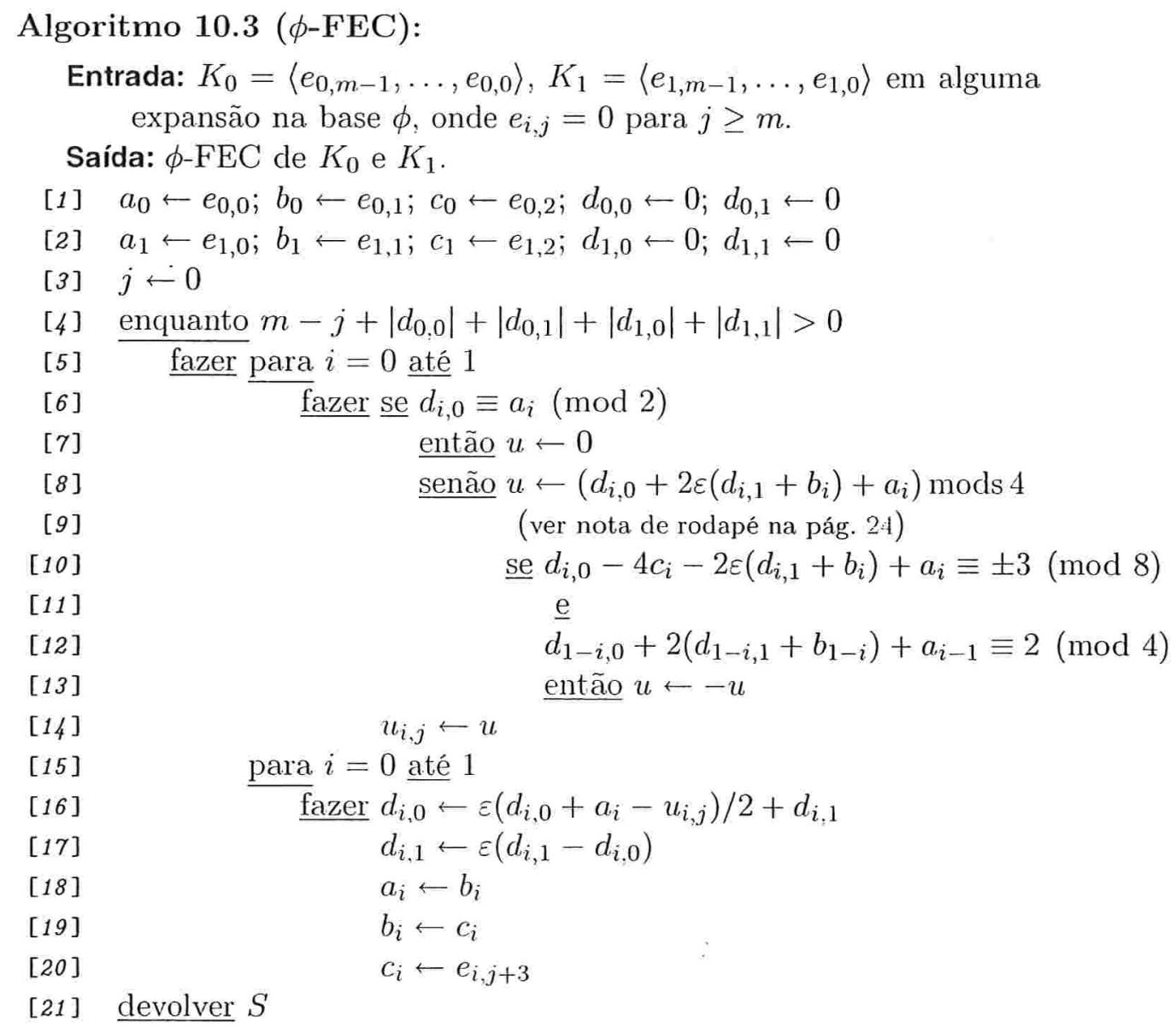

Visualize a entrada do algoritmo 10.2 como um array, onde somente a primeira e seguinda posiçōes estão ocupadas ${ }^{8}$ :

\begin{tabular}{|c|c|c|}
\hline & 1 & 0 \\
\hline$L_{0}=$ & $L_{0,1}$ & $L_{0,0}$ \\
\hline$L_{1}=$ & $L_{1,1}$ & $L_{1,0}$ \\
\hline
\end{tabular}

Se agora considerarmos agora para o algoritmo 10.3 a expansão $\phi$-ádica de $L_{i}=L_{i, 1} \phi+L_{i, 0}$ como

\begin{tabular}{|c|c|c|c|c|c|}
\hline & & & & & $L_{i, 0}$ \\
\hline & $m-1$ & $\cdots$ & $c_{i}$ & $b_{i}$ & $a_{i}$ \\
\hline$L_{0}=$ & $e_{0, m-1}$ & $\cdots$ & $e_{0,2}$ & $e_{0,1}$ & $e_{0,0}$ \\
\hline$L_{1}=$ & $e_{1, m-1}$ & $\cdots$ & $e_{1,2}$ & $e_{1,1}$ & $e_{1,0}$ \\
\hline
\end{tabular}

${ }^{3}$ Lembre-se que na entrada dos algoritmos 10.2 e 10.3 temos $d_{i}=d_{i, 0}+d_{i, 1} \phi=0$. 
e observarmos apenas os 3 últimos bits da expansão $\phi$-ádica de $L_{i}$ temos que:

$$
L_{i, 1}=\phi c_{i}+b_{i}+d_{i, 1} \quad \text { e } \quad L_{i, 0}=a_{i}+d_{i, 0}
$$

As linhas (6),(8),(10),(12) e (18 a 20) do algoritmo 10.3 equivalem as linhas $(7),(9),(11),(13)$ e (20 a 21) do algoritmo 10.2. Iremos demonstrar cada uma destas equivalências.

Linha $(7) \rightarrow$ Linha $(6)$

Como $L_{i, 0}=a_{i}+d_{i, 0}$, então $d_{i, 0} \equiv a_{i} \bmod 2$ torna $L_{i, 0}$ par.

Linha (9) $\rightarrow$ Linha (8)

$$
\left(L_{i, 0}+2 \varepsilon L_{i, 1}\right) \equiv\left(a_{i}+d_{i, 0}+2 \varepsilon\left(b_{i}+d_{i, 1}\right)\right) \bmod 4
$$

Linhas $(11) \rightarrow$ Linhas (10)

$$
\begin{gathered}
\left(L_{i, 0}-2 \varepsilon L_{i, 1}\right) \equiv \\
\equiv\left(a_{i}+d_{i, 0}-2 \varepsilon\left(\phi c_{i}+b_{i}+d_{i, 1}\right)\right) \equiv\left(a_{i}+d_{i, 0}-2 \varepsilon\left(b_{i}+d_{i, 1}-2 \varepsilon c_{i}\right)\right) \equiv \\
\equiv\left(a_{i}+d_{i, 0}-2 \varepsilon\left(b_{i}+d_{i, 1}\right)+4 c_{i}\right) \equiv\left(a_{i}+d_{i, 0}-2 \varepsilon\left(b_{i}+d_{i, 1}\right)-4 c_{i}\right) \equiv \\
\vdots \quad \\
\equiv \pm 3 \quad \bmod 8
\end{gathered}
$$

Linhas $(13) \rightarrow$ Linhas (12)

$$
\begin{gathered}
\left(L_{1-i, 0}+2 L_{1-i, 1}\right) \equiv \\
\equiv\left(a_{1-i}+d_{1-i, 0}+2\left(b_{1-i}+d_{1-i, 1}\right)\right) \equiv 2 \bmod 4
\end{gathered}
$$

Linhas (20 a 21) $\rightarrow$ Linhas (18 a 20)

A computação de $\left\lfloor\left(K_{i, 0}+K_{i, 1} \phi\right) / \phi\right\rfloor$ das linhas (18 a 20) não é nada mais do que o deslocamento dos bits da expansão $\phi$-ádica de $K_{i}$.

Pode-se adaptar o algoritmo 10.3 para aceitar como entrada pares $K_{i}=$ $z_{i, 0}+z_{i, 1} \phi$, onde $i \in\{0,1\}$, o que facilita a computação de $K_{0} P+K_{1} Q$ utilizado no processo de verificação de assinaturas digitais. 


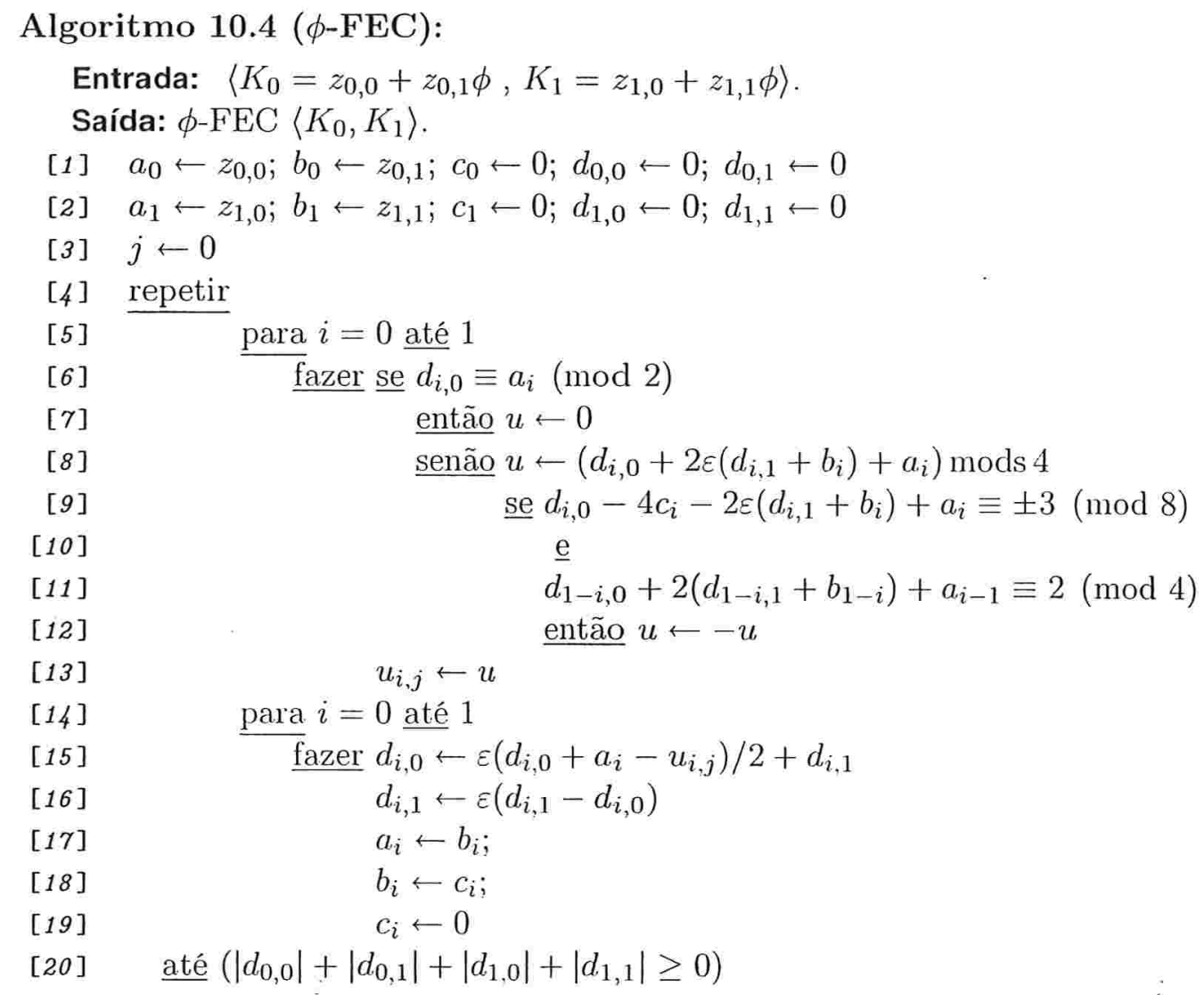

\subsection{Aplicações da $\phi$-FEC}

Existem dois casos em que a $\phi$-FEC pode ser aplicada com eficiência.

A primeira é na checagem de assinatura feita através da computação de expressões do tipo $K_{0} P+K_{1} Q$ com uso do Método rápido de Shamir

A segunda é na computação da multiplicação escalar simples $K P$

- Pré-compute $\phi^{l / 2}(P)$ e $P \pm Q$

- Obtenha a expansão $\phi$-ádica de $K$ dividindo-a em duas partes

$$
\overbrace{\sum_{i=0}^{l / 2-1} k_{i} \phi^{i}}^{K_{0}}+\phi^{l / 2} \underbrace{\sum_{i=0}^{l / 2-1} k_{i+l / 2} \phi^{i}}_{K_{1}} .
$$

- Compute, com o algoritmo $10.3, \phi$ - FEC $\left(K_{0}, K_{1}\right)$

- Método rápido de Shamir para se obter $K_{0} P+K_{1} \phi^{l / 2}(P)$. 


\subsection{Comparações: endomorfismo de Frobenius}

\section{Checagem de assinatura}

Como computar $K_{0} P+K_{1} Q$ usando a $\phi$-FEC custa $l$ aplicações de $\phi$ e $l / 2$ adições, onde $l \leq m \approx \log _{2} 2^{n}=n$ ao passo que fazer o mesmo com a $\phi$-NAF e o método rápido de Shamir custa $l$ aplicações de $\phi$ e $5 l / 9$ adições, usar a $\phi$-FEC é melhor do que usar um joint $\phi$-NAF.

Por outro lado, como a aplicação do endomorfismo é muito rápido do que uma duplicação, utilizar a $\phi$-FEC é muito mais rápido do que usar a FEC de $K_{0}, K_{1}$.

\begin{tabular}{|c|c|c|}
\hline \multicolumn{3}{|c|}{$\phi$ é Frobenius: checagem de assinatura } \\
\hline \hline FEC & joint $\phi$-NAF & $\phi$-FEC \\
\hline$(m) D$ e $(m / 2) A$ & $(m) \phi$ e $(5 m / 9) A$ & $(m) \phi$ e $(m / 2) A$ \\
\hline Pré-computação de & Pré-computação de & Pré-computação de \\
$P \pm Q$ & $P \pm Q$ & $P \pm Q$ \\
\hline
\end{tabular}

\section{Multiplicação simples}

Como computar $K P$ usando a $\phi$-FEC custa $l / 2$ vezes $\phi$ e $l / 4$ adições, onde $l \leq m \approx \log _{2} 2^{n}=n$, ao passo que fazer mesmo com a $\phi$-ádica de Solinas requer $l$ vezes $\phi$ e $l / 3$ adições, a aplicação da $\phi$-FEC é a abordagem mais rápida.

\begin{tabular}{|c|c|}
\hline \multicolumn{2}{|c|}{$\phi$ é Frobenius: multiplicação simples } \\
\hline \hline Expansão NAF $\phi$-ádica de Solinas & $\phi$-FEC \\
\hline$(m) \phi$ e $(m / 3) A$ & $(m / 2) \phi$ e $(m / 4) A$ \\
\hline & Pré-computação de $\phi^{l / 2}(P)$ e \\
& $P \pm \phi^{l / 2}(P)$ \\
\hline
\end{tabular}

\subsection{Comparações: endomorfismo da curva do ex- emplo 10.1}

\section{Checagem de assinatura}

Uma abordagem é aplicar a redução GLV em $K_{i}$ produzindo $\left\langle z_{i, 0}, z_{i, 1}\right\rangle$ $(i \in\{0,1\})$ que é usado como entrada do Algoritmo 10.4, resultando em um custo de $l \phi$ e $l / 2$ adições, onde $l \leq m \approx \log _{2} \eta$. A outra, mais custosa, é aplicar a FEC na expansão binária de $K_{i}$ que custa $l$ duplicações e $l / 2$ adições.

\begin{tabular}{|c|c|}
\hline \multicolumn{2}{|c|}{$\phi$ é endomorfismo dos exemplos: checagem de assinatura } \\
\hline \hline FEC & $\phi$-FEC \\
\hline$(m) \mathrm{D}$ e $(m / 2) \mathrm{A}$ & $(m) \phi$ e $(m / 2) \mathrm{A}$ \\
(pré-computação de $P \pm Q)$ & (pré-computação de $\phi(P), \phi(Q)$ e \\
& $P \pm Q)$ \\
\hline
\end{tabular}




\section{Multiplicação simples}

Usar a $\phi$-FEC custa $l / 2 \phi$ e $l / 4$ adições, onde $l \leq m \approx \log _{2} \eta$, ao passo que efetuar a redução GLV de $K$ obtendo $\left\langle z_{0}, z_{1}\right\rangle$, aplicar a FEC e em seguida aplicar o método rápido de Shamir, custa $l / 2$ duplicações e $l / 4$ adições. Como duplicações são mais custosas, a primeira abordagem é mais rápida.

\begin{tabular}{|c|c|}
\hline \multicolumn{2}{|c|}{$\phi$ é endomorfismo dos exemplos: multiplicação simples } \\
\hline \hline Método GLV & $\phi$-FEC \\
\hline$(m / 2) \mathrm{D}$ e $(m / 4) \mathrm{A}$ & $(m / 2) \phi$ e $(m / 4) \mathrm{A}$ \\
$($ pré-computação de $\phi(P)$ e & $\left(\right.$ pré-computação de $\phi^{l / 2}(P)$ e \\
$\left.P \pm \phi^{l / 2}(P)\right)$ & $\left.P \pm \phi^{l / 2}(P)\right)$ \\
\hline
\end{tabular}

\subsection{Obtenção da $\phi$-FEC na curva do exemplo 10.2}

Não se pode obter a $\phi$-FEC para a curva do exemplo 10.2, pela aplicação direta o algoritmo 10.3 pelo fato deste algoritmo ter sido construído a partir do algoritmo 6.3 que computa a expansão $\phi$-NAF em curvas com endomorfismo com equação característica $\phi^{2}-\varepsilon \phi+2=0$.

Assim sendo, na a curva do exemplo 10.2, a $\phi$-FEC é obtida, por partes, a partir do método de obtenção de uma expansão $\phi$-NAF nestas curvas, descrito na seção 10.1.2, ou seja:

Checagem de assinatura

- Faça a redução GLV de $K_{0}$ obtendo $K_{0} \equiv z_{0,0}+z_{0,1} \phi(\bmod \eta)$

- Obtenha a NAF $\phi$-ádica de $z_{0,0}=\sum_{i=0}^{l_{0}-1} u_{i} \phi^{i}$

- Obtenha a NAF $\phi$-ádica de $z_{0,1}=\sum_{i=0}^{l_{1}-1} v_{i} \phi^{i}$

- Aplique a $\phi$-FEC $\left(z_{0,0}, z_{0,1}\right)$ com algoritmo 10.3

- Pré-compute $\phi(P)$ e $P \pm \phi(P)$

- $R=z_{0,0} P+z_{0,1} \phi(P)$

- Faça a redução GLV de $K_{1}$ obtendo $K_{1} \equiv z_{1,0}+z_{1,1} \phi(\bmod \eta)$

- Obtenha a NAF $\phi$-ádica de $z_{1,0}=\sum_{i=0}^{l_{0}-1} u_{i}^{\prime} \phi^{i}$

- Obtenha a NAF $\phi$-ádica de $z_{1,1}=\sum_{i=0}^{l_{1}-1} v_{i}^{\prime} \phi^{i}$

- Aplique a $\phi$-FEC $\left(z_{1,0}, z_{1,1}\right)$ com algoritmo 10.3

- Pré-compute $\phi(Q)$ e $P \pm \phi(Q)$

- $S=z_{1,0} Q+z_{1,1} \phi(Q)$ 
- $K P=R+S$

- Custo : $(m / 2) A+(m) \phi$ onde $m \leq \log _{2} \eta$

\section{Multiplicação simples}

- Faça a redução GLV de $K$ obtendo $K \equiv z_{0}+z_{1} \phi(\bmod \eta)$

- Obtenha a NAF $\phi$-ádica de $z_{0}=\sum_{i=0}^{l_{0}-1} k_{i} \phi^{i}=\overbrace{\sum_{i=0}^{l_{0} / 2-1} k_{i} \phi^{i}}^{K_{0}}+\phi^{l_{0} / 2} \overbrace{\sum_{i=0}^{l_{0} / 2-1} k_{i+l_{0} / 2} \phi^{i}}^{K_{1}}$

- Pré-compute $\phi^{l_{0} / 2}(P)$ e $P \pm \phi^{l_{0} / 2}(P)$

- Aplique a $\phi$-FEC $\left(K_{0}, K_{1}\right)$ com algoritmo 10.3

- $R=K_{0} P+K_{1} \phi^{l_{0} / 2}(P)$

- Obtenha a NAF $\phi$-ádica de $z_{1}=\sum_{i=0}^{l_{1}-1} k_{i} \phi^{i}=\overbrace{\sum_{i=0}^{l_{1} / 2-1} k_{i} \phi^{i}}^{K_{0}}+\phi^{l_{1} / 2} \overbrace{\sum_{i=0}^{l_{1} / 2-1} k_{i+l_{1} / 2} \phi^{i}}^{K_{1}}$

- Pré-compute $\phi^{l_{1} / 2}(P)$ e $P \pm \phi^{l_{1} / 2}(P)$

- Aplique a $\phi$-FEC $\left(K_{0}, K_{1}\right)$ com algoritmo 10.3

- $S=K_{0} P+K_{1} \phi^{l_{1} / 2}(P)$

- $K P=R+S$

- Custo: $(m / 4) A+(m / 2) \phi$ onde $l_{0}, l_{1} \leq m \leq \log _{2} \eta$

\subsection{Conclusão}

Neste capítulo foi mostrado, em primeiro lugar, como os autores [ estenderam a expansão $\phi$-ádica das curvas de Koblitz, como descrito no capítulo (i para uma classe de curvas definidas sobre corpos primos que possuem endomorfismos computáveis eficientemente.

Em seguida, foi mostrado como obter a $\phi$-FEC, uma expansão na base $\phi$ conjunta com sinal de $K_{0}$ e $K_{1}$ que acelera a computação da multiplicação dupla $K_{0} P+K_{1} Q$ em algumas curvas elípticas definidas sobre corpos primos (exemplos 10.1 e 10.2) e curvas binárias anômalas, bem como acelerar a multiplicação simples $K P$ nas mesmas.

No que se refere às curvas dos exemplos 10.1 e 10.2, fizemos as seguintes contribuições: 
- Mostramos como transformá-las na forma simplificada de uma curva definida sobre um corpo primo, ou seja, mostramos como transformar

$$
y^{2}=x^{3}-(3 / 4) x^{2}-2 x-1 \text { em } y^{2}=x^{3}-\frac{35}{16} x^{2}-\frac{49}{32}
$$

bem como transformar

$$
y^{2}=4 x^{3}-30 x-28 \quad \text { em } \quad y^{2}=x^{3}-120 x-448
$$

Tal transformação tem como objetivo poder usá-las na implementação.

- Mostramos como obter parâmetros das referidas curvas utilizando o software MAGMA [1].

- Ressaltamos que nem todos os valores de $p$, obtidos conforme explicitado nos exemplos 10.1 e 10.2, são úteis para aplicações criptográficas, pois na maioria das vezes é necessário fazer a aplicação do endomorfismo mais de uma vez, ou seja, temos que $\phi^{u}(P)=\lambda P(u \geq 1)$ e não $\phi(P)=\lambda P$ como era de se esperar. No Apêndice B mostramos um desses casos.

- Apresentamos no referido apêndice alguns $p$ que requerem apenas uma aplicação do endomorfismo.

Nossa contribuição, dentro da primeira proposição de estender a expansão NAF $\phi$-ádica das curvas de Koblitz, foi detalhar a obtenção da expansão $\phi$-ádica das curvas definidas nos exemplos, da seguinte maneira:

- Inserimos notas e esquemas explicativos ao longo da seção 10.1.2.

- Detalhamos a obtenção de uma expansão $\phi$-ádica para a curva do exemplo 10.2, culminando com a apresentação do algoritmo 10.1 .

Contribuímos também ao detalhar a construção do algoritmo 10.3, que obtém a $\phi$-FEC, da seguinte forma:

- Inserindo notas ao longo da seção 10.2 .

- Explicando a obtenção da expansão NAF $\phi$-ádica de Solinas para a curva do exemplo 10.2 (seção 10.1.2).

- Apresentando o algoritmo 10.2 que computa $\phi$-FEC a partir dos inteiros $K_{0}$ e $K_{1}$. O referido algoritmo é uma modificação do algoritmo 6.3 que computa a $\phi$-NAF .

- Deduzindo o algoritmo 10.3 a partir do algoritmo 10.2 .

- Mostrando, na seção 10.6, como aplicar $\phi$-FEC na curva do exemplo 10.2 . 


\section{Capítulo 11}

\section{Conclusões}

Alcançamos nosso objetivo ao estudarmos os principais métodos que aceleram a multiplicação escalar elíptica. De modo subjacente apresentamos uma evolução histórica, o que torna esse trabalho proveitoso para os que pretendem pesquisar novas técnicas. Uniformizamos também a simbologia usada pelos autores.

Implementamos a maioria dos algoritmos ${ }^{1}$. Nessa implementação, utilizamos a biblioteca $\mathrm{C} / \mathrm{C}++$ MIRACL [1:], o que permitiu, em parte, confirmar os resultados esperados. A referida biblioteca, infelizmente, ainda não implementa bases normais, somente as polinomiais.

Na obtenção dos parâmetros das curvas dos exemplos 10.1 e 10.2 utilizamos o software MAGMA [ ] que possui uma interface web disponível na internet (ver Apendice $B$ ).

Assumimos que $P$ e $Q$ possuam ordem $\eta \leq \mathcal{N}_{n}$ e, portanto, os multiplicadores são aleatoriamente selecionados de inteiros no intervalo $[1, \eta]$. Utilizamos também a seguinte simbologia: $A=$ adição, $D=$ duplicação e $\phi$ e $\varphi$ que são, respectivamente, aplicações dos endomorfismos $\phi$ e $\varphi$ (Frobenius).

Fizemos uma comparação teórica dos algoritmos e nesta comparação consideramos que a implementação da aritmética dos corpos (primos e binários) são otimizadas e os corpos binários são implementados em base normal, de tal forma que o custo da aplicação do endomorfismo de Frobenius seja inexpressível.

Fizemos duas grandes divisões: multiplicação simples $(K P)$ e multiplicação dupla $\left(K_{0} P+K_{1} Q\right)$.

Na multiplicação simples fizemos a seguinte subdivisão de acordo com o tipo de curva usada: métodos gerais, métodos aplicados em curvas binárias anômalas, métodos aplicados em curvas definidas sobre pequenos corpos de característica 2 e métodos aplicados em curvas definidas sobre corpos primos.

Na multiplicação dupla fizemos a seguinte subdivisão, também de acordo

\footnotetext{
${ }^{1}$ Os que não foram implementados encontram-se no capítulo 3
} 
com o tipo de curva usada: métodos aplicados em curvas binárias anômalas e métodos aplicados em curvas definidas sobre corpos primos.

Dentro dessas divisões ( e subdivisões), comparamos os os métodos levando em consideração três grandes casos:

- Na computação da multiplicação simples $K P$, não se pode usar a vantagem de $P$ ser conhecido antecipadamente, por que não o é, e portanto qualquer pré-computação deve ser "on-line"2;

- Na computação da multiplicação simples $K P$, pode-se usar a vantagem de $P$ ser conhecido antecipadamente (uma vez que $P$ é fixo) e portanto qualquer pré-computação pode ser feita"off-line".

- Na computação da multiplicação dupla $K_{0} P+K_{1} Q$ pode-se usar somente a vantagem de $P$ se conhecido antecipadamente.

Os últimos dois casos são motivados por protocolos tais como ECDSA, onde a geração de uma assinatura requer a computação $K P$ onde $P$ é fixo, e a verificação de uma assinatura requer uma computação do tipo $K_{0} P+K_{1} Q$ onde $P$ é fixo e $Q$ não é conhecido "à priori".

\subsection{Multiplicação simples}

\subsubsection{Métodos gerais de multiplicação}

Apresentamos no capítulo 3 métodos que são aplicáveis em qualquer curva elíptica e não tomam proveito de qualquer característica da mesma.

Ao contrário dos outros métodos, a técnica embutida no método (5) considera que $P$ é fixo (conhecido "à priori") e portanto o custo da précomputação não foi embutida no custo do algoritmo ${ }^{3}$. Os métodos (1) e (2) usam o conceito de cadeia aditiva (seção 3.1) e os métodos (3) e (4) o de seqüência aditiva (seção 3.2).

Na tabela a seguir, $m \approx \log _{2} \eta$.

Para exemplificar, vamos considerar dois exemplos, um para uma curva definida sobre um corpo binário, e outra para uma curva definida sobre um corpo primo. Neste dois exemplos, a pré-computação é "off-line"; $w=3$ e usa-se o sistema de coordenada projetiva padrão (ver tabelas 2.1 e 2.2)

\footnotetext{
${ }^{2} \mathrm{O}$ fato da pré-computação ser "on-line"ou "off-line"está relacionada ao número de vezes que a mesma tem de ser efetuada. Se é "on-line"tem de ser executada toda vez que se fizer a multiplicação. Se é "off-line"é executada apenas uma vez, e portanto, não embutimos no custo do método

${ }^{3} \mathrm{O}$ método BGMW foi concebido para trabalhar com ponto $P$ fixo e, portanto, é ineficiente nos casos onde $P$ não seja conhecido "à priori"devido ao alto custo da précomputaçāo
} 
Seja uma curva definida sobre $\mathbb{F}_{2}$ e considerada sobre o corpo extendido $\mathbb{F}_{2^{163}}$, um ponto $P$ (fixo) e $K \in[0, \eta]$, onde

$$
\eta=5846006549323611672814741753598448348329118574063,
$$

e $m=\log _{2} \eta=162$ bits. Neste caso, o BMGW é o melhor método:

- Binário: $(m / 2)(13 M)+(m)(7 M)=13,5 m M=2187 M$

- NAF Binário: $(m / 3)(13 M)+(m)(7 M)=11,3 m M=1831 M$

- $2^{w}$-ário NAF: $(m / 4)(13 M)+(m)(7 M)=10,25 M=1660 M$

- Janela Deslizante: $(m / 4,5)(13 M)+(m)(7 M)=9,88 M=1601 M$

- BGMW: $(59)(13 M)=767 M$

Agora considere uma curva definida sobre um corpo primo, um ponto $P$ (fixo), $K \in[0, \eta], m=\log _{2} \eta=162$ bits e $S / M=0,8$. Também neste caso o BMGW é o melhor método :

- Binário: $(m / 2)(13,6 M)+(m)(10,2 M)=17 m M=2754 M$

- NAF Binário: $(m / 3)(13,6 M)+(m)(10,2 M)=14,7 m M=2381 M I$

- $2^{w}$-ário NAF: $(m / 4)(13,6 M)+(m)(10,2 M I)=13,6 M=2203 M$

- Janela Deslizante: $(m / 4,5)(13,6 M)+(m)(10,2 M)=13,22 M=2141 M$

- BGMW: $(59)(13,6 M)=802 M$ 


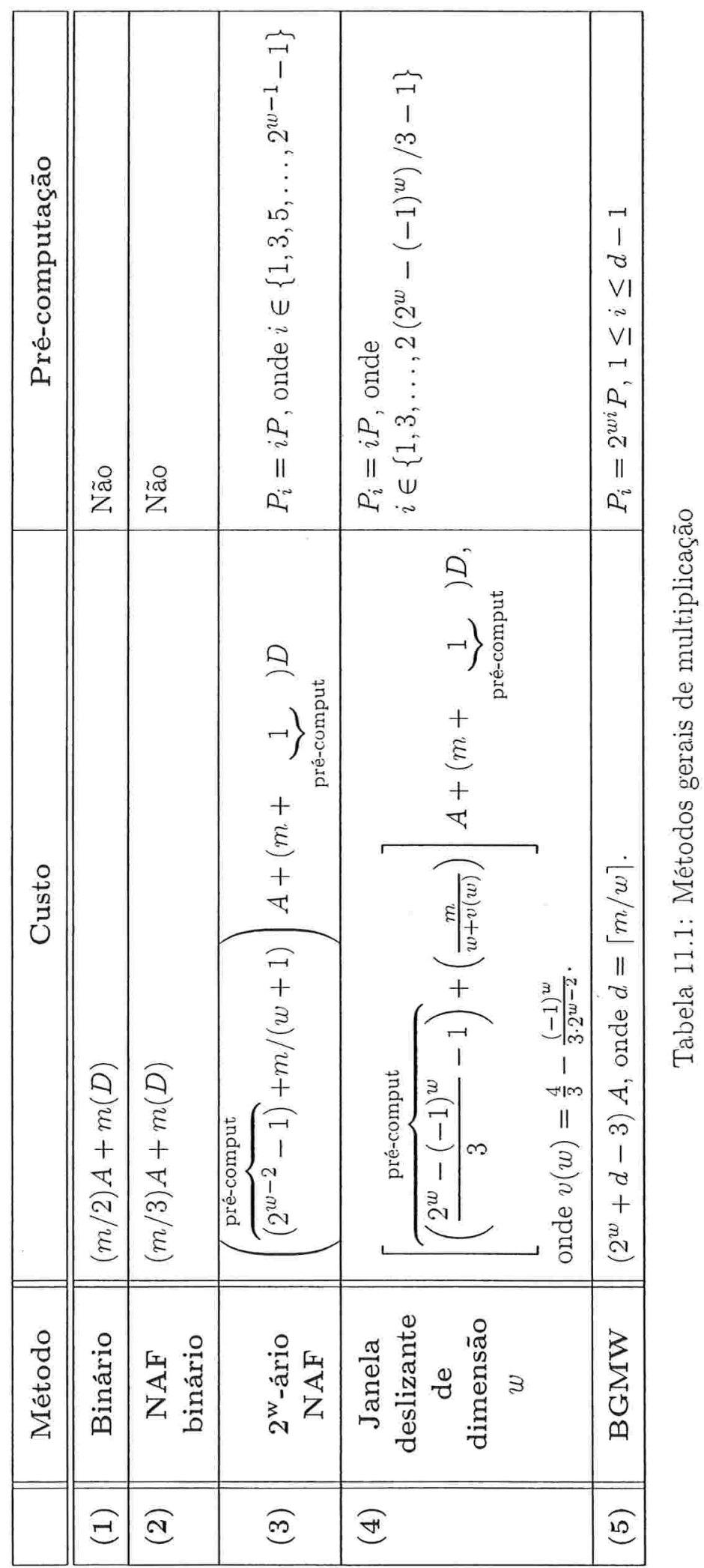




\subsubsection{Multiplicação em curvas binárias anômalas}

Seja a curva elíptica $E^{1}: y^{2}+x y=x^{3}+x^{2}+1$ e seu twist $E^{0}: y^{2}+x y=x^{3}+1$ definidas sobre $\mathbb{F}_{2}$.

Como esses métodos usam a propriedade de que, com o uso de bases normais, a computação do endomorfismo de Frobenius $(\varphi)$ nestas curvas possui um custo computacional desprezível, consideramos na tabela abaixo o custo de $\varphi$ aproximadamente igual a 0 . Portanto, os valores pré-computados não influem no custo do algoritmo. Nesta tabela $m=n \quad 4$.

Tomando por base estas considerações, o melhor método é o (8).

Os métodos (6) e (7) usam o conceito de cadeia aditiva.

Para exemplificar a vantagem do uso do endomorfismo de Frobenius, vamos considerar a curva definida na seção 11.1.1. Neste caso, o método (6) já é mais eficiente que o BGMW, pois possui um custo de 1059, $5 \mathrm{M}$.

\begin{tabular}{|c|c|c|c|}
\hline & Método & Custo & Pré-computação \\
\hline (9) & $\begin{array}{l}\text { Volker Müller com } \\
\qquad \mathbb{F}_{2}\end{array}$ & $\overbrace{m \varphi}^{\approx 0}+(m) A$ & Não \\
\hline (6) & Meier e Staffelbach & $\overbrace{m \varphi}^{\approx 0}+(m / 2) A$ & Não \\
\hline (7) & $\begin{array}{c}\text { Expansão NAF } \\
\phi \text {-ádica de Solinas }\end{array}$ & $\overbrace{m \varphi}^{\approx 0}+(m / 3) A$ & Não \\
\hline (8) & $\begin{array}{c}\text { Expansão } \varphi \text {-FEC de } \\
\text { Ciet, Lange, Sica e } \\
\text { Quisquater }\end{array}$ & $\underbrace{(m / 2) \varphi}_{\approx 0}+(m / 4) A$ & $\overbrace{\varphi^{1 / 2}(P)}^{\approx 0}$ e $P \pm \underbrace{\varphi^{l / 2}(P)}_{\approx 0}$ \\
\hline
\end{tabular}

Tabela 11.2: Multiplicação em curvas binárias anômalas

\subsubsection{Multiplicação em curvas definidas em pequenos cor- pos de característica 2}

Como esse método usa a propriedade de que, como o uso de bases normais, a computação do endomorfismo de Frobenius $(\varphi)$ nesta curva possui um custo computacional desprezível, consideramos na tabela abaixo o custo de $\varphi$ aproximadamente igual a 0 . Portanto, o valor pré-computado não influe no custo do algoritmo.

A grande vantagem deste método é permitir o uso do endomorfismo de Frobenius em uma classe mais ampla de curvas definidas sobre corpos de

\footnotetext{
${ }^{4}$ Vimos, nos capítulos correspondentes, que $K$ deve ser reduzido à módulo $\left(\varphi^{n}-1\right)$ antes de se aplicar os métodos desta seção e portanto $m=n$.
} 
característica 2, em não somente sobre o corpo $\mathbb{F}_{2}$.

Este método usa o conceito de seqüência aditiva.

\begin{tabular}{|c||c||c|c|}
\hline & Método & Custo & Pré-computação \\
\hline \hline \multirow{2}{*}{$(9)$} & $\begin{array}{c}\text { Volker } \\
\text { Müller }\end{array}$ & $(m) \varphi+\overbrace{(q / 2}^{\approx 0}+m-1) A$ onde $m \leq n+3$ & $\{1, \ldots, q / 2\}$ \\
\hline
\end{tabular}

Tabela 11.3: Multiplicação em curvas definidas em pequenos corpos de característica 2

\subsubsection{Multiplicação em curvas definidas sobre corpos pri- mos}

Se considerarmos que o ponto $P$ é fixo, então , como a aplicação do endomorfismo $\phi$ é mais rápido do que uma duplicação (se considerarmos o uso de coordenadas projetivas), mas não mais rápido do que duas duplicações, o método (12) é o que possui menor custo para a curva do exemplo 10.1 e o método (13) o menor custo para a curva do exemplo 10.2. Caso contrário, como a aplicação do endomorfismo de Frobenius nestas curvas não é desprezível, o custo envolvido com a pré-computação nos métodos (12) e (13) reduzem sua eficiência e o melhor método passa a ser o (11c).

Nas outras curvas definidas sobre corpos primos, o melhor método é o (11c) independentemente do ponto $P$ ser fixo ou não.

Na tabela abaixo $m=\log _{2} \eta \quad 5$.

Para exemplificar, sejam os métodos (11c), (12), (13) e consideremos a curva definida sobre um corpo primo descrita na seção $11.1 .1^{6}$, bem como a pré-computação "off-line":

- (11c): $(\mathrm{m} / 2)(10,2 \mathrm{M})+(\mathrm{m} / 4)(13,6 \mathrm{M})=8,5 \mathrm{mM}=1377 \mathrm{M}$

- $(12):(m / 2)(9,6 M)+(m / 4)(13,6 M)=8,2 m M=1328 M$

- (13): $(m / 2)(7,6 M)+(m / 4)(13,6 M)=7,2 m M=1166 M$

Este resultado mostra que, para este caso, os métodos supracitados só não são melhores que o BGMW ${ }^{7}$.

\footnotetext{
${ }^{5}$ Os métodos descritos nesta seção usam a redução GLV de $K$ par se obter $K \equiv$ $\left(z_{0}+z_{1} \lambda\right) \bmod \eta$, onde $z_{0}, z_{1} \in[0,[\sqrt{\eta}\rfloor]$ e portanto $m=\log _{2} \eta$.

${ }^{6}$ Supor que a mesma atenda, nos métodos (12) e (13), respectivamente, aos requisitos dos exemplos 10.1 e 10.2

${ }^{7}$ Ver na seção supracitada considerações relativas a esse método
} 


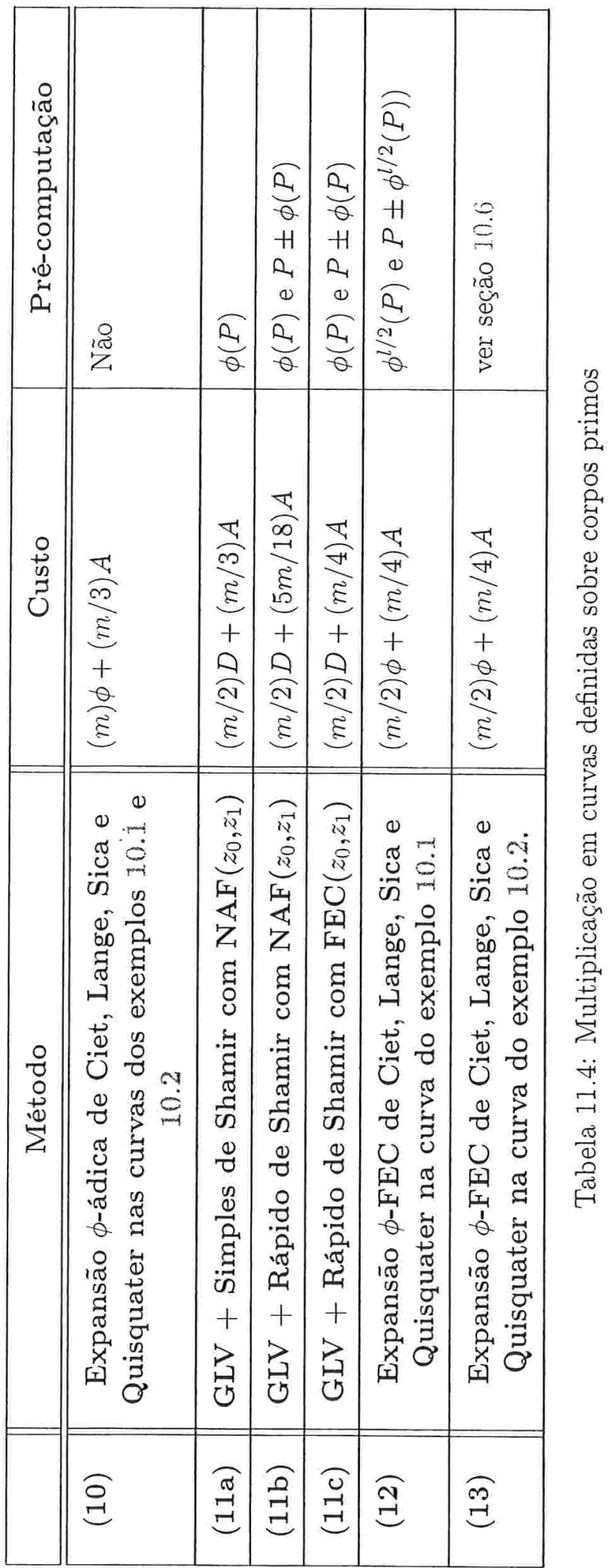




\subsection{Multiplicação dupla}

\subsubsection{Multiplicação dupla em curvas binárias anômalas}

Seja $E^{1}: y^{2}+x y=x^{3}+x^{2}+1$ e seu twist $E^{0}: y^{2}+x y=x^{3}+1$ definidas sobre $\mathbb{F}_{2}$. Deseja-se computar $K_{0} P+K_{1} Q$, que é usado principalmente na verificação de assinaturas digitais.

Como a aplicação de $\varphi$ possui custo desprezível, o método (14b) é o mais eficiente.

Na tabela abaixo, $m=n \quad 8$.

\begin{tabular}{|c||c||c|l|}
\hline & Método & Custo & Pré-computação \\
\hline \hline$(13)$ & $\begin{array}{c}\text { Simples de Shamir } \\
\text { com NAF }\left(K_{0}, K_{1}\right)\end{array}$ & $m D+(2 m / 3) A$ & Não \\
\hline (14a) & $\begin{array}{c}\text { Rápido de Shamir } \\
\text { com FEC }\left(K_{0}, K_{1}\right)\end{array}$ & $m D+(m / 2) A$ & $P \pm Q$ \\
\hline (14b) & $\begin{array}{c}\text { Rápido de Shamir } \\
\text { com } \varphi \text {-FEC }\left(K_{0}, K_{1}\right)\end{array}$ & $\overbrace{m \varphi}^{\approx 0}+(m / 2) A$ & $P \pm Q$ \\
\hline
\end{tabular}

Tabela 11.5: Multiplicação dupla em curvas binárias anômalas

\subsubsection{Multiplicação dupla em curvas definidas sobre corpos primos}

Deseja-se computar $K_{0} P+K_{1} Q$ que é utilizado principalmente na verificação de assinaturas digitais.

Como a aplicação do endomorfismo $\phi$ é mais rápido do que uma duplicação (se considerarmos o uso de coordenadas projetivas), o método (16b) é o que possui menor custo para a curva do exemplo 10.1 e o método (16c) o menor custo para a curva do exemplo 10.2.

Nas outras curvas definidas sobre corpos primos, o melhor método é o (16a).

Na tabela abaixo $m=\log _{2} \eta \quad 9$.

\footnotetext{
${ }^{8}$ Nos métodos (13) e (14a), $m=\log _{2} \eta \leq \log _{2} \mathcal{N}_{n} \approx \log _{2} 2^{n}=n$. No (14b), $K_{i}$ deve ser reduzido à módulo $\left(\varphi^{n}-1\right)$ antes da aplicação do método e portanto $m=n$.

${ }^{9}$ Os métodos (16b) e (16c) usam a redução GLV de $K_{i}$ para se obter $K_{i} \equiv\left(z_{i, 0}+\right.$ $\left.z_{i, 1} \lambda\right) \bmod \eta$, onde $\left\{z_{i, 0}, z_{i, 1}\right\} \in[0,\lfloor\sqrt{\eta}\rfloor]$ e portanto $m=\log _{2} \eta$.
} 


\begin{tabular}{|c||c||c|l|}
\hline & Método & Custo & Pré-computação \\
\hline \hline (16) & $\begin{array}{c}\text { Simples de Shamir } \\
\text { com NAF }\left(K_{0}, K_{1}\right)\end{array}$ & $m D+(2 m / 3) A$ & Não \\
\hline (16a) & $\begin{array}{c}\text { Rápido de Shamir } \\
\text { com FEC }\left(K_{0}, K_{1}\right)\end{array}$ & $m D+(m / 2) A$ & $P \pm Q$ \\
\hline (16b) & $\begin{array}{c}\text { Rápido de Shamir } \\
\text { na curva do } \\
\text { exemplo } 10.1 \text { com } \\
\phi \text {-FEC }\left(K_{0}, K_{1}\right)\end{array}$ & $m \phi+(m / 2) A$ & $\phi(P), \phi(Q)$ e $P \pm Q$ \\
\hline (16c) & $\begin{array}{c}\text { Rápido de Shamir } \\
\text { na curva do } \\
\text { exemplo } 10.2 \text { com } \\
\phi \text {-FEC }\left(K_{0}, K_{1}\right)\end{array}$ & $m \phi+(m / 2) A$ & ver seção 1.0 .6 \\
\hline
\end{tabular}

Tabela 11.6: Multiplicação dupla em curvas definidas sobre corpos primos 


\section{Apêndice A}

\section{Conceitos básicos}

Quando não citado explicitamente as definições foram extraídas de extraídas de [25].

\section{Notação $O(n)$ e $o(n)$}

Vamos considerar somente funções $f(n)$ definidas sobre inteiros $n>0$, com valores reais $f(n)>0$ para infinitos $n$.

Escrevemos $f(n)=O(n)$ se existe uma constante $c>0$ e um inteiros $n_{0}$ tais que $0 \leq f(n) \leq c g(n)$ para todo o $n>n_{0}$, ou seja, para $n$ suficientemente grande, $f(n)$ não cresce mais do que $g(n)$, a menos de um fator constante. Por exemplo, $12 n^{3}+300 n+21000=O\left(n^{3}\right)$ e $300 e^{3 n+5}+e^{n+400}+$ $n^{200}=O\left(e^{3 n+5}\right)$.

Escrevemos $f(n)=o(n)$ se para qualquer constante $c>0$ existe um inteiro $n_{0}>0$ tal que $0 \leq f(n)<c g(n)$ para todo o $n>n_{0}$, ou seja, para $n$ suficientemente grande, $f(n)$ é insignificante em relação a $g(n)$, menos de um fator constante. Em particular $f(n)=o(1)$ quando $f(n)$ tende a zero quando $n$ tende ao infinito.

\section{Problema do logaritmo discreto}

Alguns criptosistemas são baseados na dificuldade do problema computacional do problema do logaritmo discreto: dado um primo $p$ e inteiros $0<g, t<p$, calcular um inteiro $s$ tal que $t=g^{s} \bmod p$.

Por exemplo, vamos supor que $p=17, g=7$ e $t=10$. Uma solução, para este caso, será $s=9$. Quando $p$ é relativamente longo (por exemplo, 1024 bits), ninguém até hoje descobriu um algoritmo eficiente, isto é, de tempo polinomial, para resolver este problema, ou seja, este problema é computacionalmente inviável de ser resolvido. 


\section{Protocolo de troca de chaves Diffie-Hellman}

Este protocolo de combinação de chaves secretas, publicado antes do RSA, foi desenvolvido por W. Diffiee e M. Hellman e é baseado no Problema do Logaritmo Discreto. Ele estabelece uma chave secreta entre duas pessoas que pretendem se comunicar. Abaixo estão os passos do protocolo para a combinação de uma chave secreta entre $A$ e $B$ :

1. $A$ escolhe um número aleatório $S_{A}, \quad 1 \leq S_{A} \leq p-2$;

2. $B$ escolhe um número aleatório $S_{B}, \quad 1 \leq S_{B} \leq p-2$;

3. $A$ calcula $t_{A}=g^{S_{A}} \bmod p$ e envia para $B$;

4. $B$ calcula $t_{B}=g^{S_{B}} \bmod p$ e envia para $A$;

5. $A$ calcula $\left(t_{B}\right)^{S_{A}} \bmod p=K_{A B}$ $\left(\right.$ Note que $\left.\left(t_{B}\right)^{S_{A}}=\left[g^{S_{B}} \bmod p\right]^{S_{A}}=g^{S_{B} S_{A}} \bmod p\right)$;

6. $B$ calcula $\left(t_{A}\right)^{S_{B}} \bmod p=K_{A B}$

(Note que $\left(t_{A}\right)^{S_{B}}=\left[g^{S_{A}} \bmod p\right]^{S_{B}}=g^{S_{A} S_{B}} \bmod p$ )

Percebe-se que o Problema do Logaritmo Discreto tem a função de proteger as chaves secretas de $A$ e $B$. Após a execução deste protocolo, $A$ e $B$ poderiam se comunicar trocando informações através de um algoritmo de chave secreta.

\section{Aritmética modular}

Seja $a$ um inteiro e $n$ um inteiro positivo. Denota-se por $a \bmod n$ o resto que é obtido quando $a$ é dividido por $n$.

Segue desta definição que $a \bmod n$ é o inteiro $r$ tal que $a=q \cdot n+r$ e $r<n$.

Exemplo

$$
\begin{aligned}
17 \bmod 5 & =2 & & (17=3 \times 5+2) \\
-133 \bmod 9 & =2 & & (-133=-15 \times 9+2) \\
2001 \bmod 101 & =82 & & (2001=19 \times 101+82)
\end{aligned}
$$

\section{Pequeno teorema de Fermat}

Seja $p$ um número primo. Se o $\operatorname{mdc}(a, p)=1$, então $a^{p-1} \equiv 1(\bmod p) . \operatorname{Em}$ particular, $a^{p} \equiv a(\bmod p)$ para todos os inteiros $a$. 


\section{Grupo}

Um grupo $(G, \circ)$ é um conjunto $G$ com uma operação binária o definida sobre $G$, tal que:

1. A operação o é associativa, i.e.,

$$
\forall a, b, c \in G: a \circ(b \circ c)=(a \circ b) \circ c ;
$$

2. Existe um elemento $n \in G$, chamado identidade ou elemento neutro, tal que

$$
\forall a \in G: a \circ n=n \circ a=a
$$

3. Para cada elemento $a \in G$ existe um elemento $\bar{a} \in G$, o inverso de $a$, tal que

$$
a \circ \bar{a}=\bar{a} \circ a=n .
$$

Dizemos que $G$ é comutativo (ou abeliano) se $\forall a, b \in G: a \circ b=b \circ a$. Se a operação o for uma multiplicação (*). o grupo é chamado grupo multiplicativo, a identidade é representada por 1 e o inverso de $a$ é representado por $a^{-1}$. Se a operação o for uma soma $(+)$, o grupo é chamado grupo aditivo, a identidade é representada por 0 e o inverso de $a$ é representado por $-a$. Um exemplo de grupo aditivo é o conjunto $\mathbb{Z}$ dos inteiros com a operação de soma usual. Outro exemplo é o conjunto $\mathbb{Z}_{n}$ dos inteiros $\bmod n$, com a soma $\bmod n$.

Para as definições a seguir considere que estamos trabalhando com grupos a.ditivos.

Se o número de elementos de $G$ for finito, tal número é chamado de ordem de $G$. Se existe um elemento $g \in G$ tal que qualquer elemento $x \in G$ possa ser escrito como um múltiplo escalar de $G$, i.e., $x=r g$ para algum inteiro $r$, então dizemos que $G$ é cíclico e $g$ é o gerador de $G$.

A ordem de um elemento $x \in G$ é o menor inteiro $r$ tal que $r x=0$. Se não existir esse elemento, dizemos que a ordem de $x \in G$ é infinita.

Se $G$ tem ordem prima, $G$ é cíclico e qualquer $x \in G$ é um gerador de $G$ de ordem $r$.

\section{Anel}

Um anel $(A,+, *)$ é um conjunto $A$ com duas operações binárias: soma $(+)$ e multiplicação $(*)$ definidas sobre $G$, tais que:

1. $(A,+)$ é um grupo aditivo comutativo com identidade aditiva 0 ;

2. A operação * é associativa, i.e.,

$$
\forall a, b, c \in A: a *(b * c)=(a * b) * c ;
$$


3. Existe uma identidade multiplicativa $1 \in A, 1 \neq 0$, tal que

$$
\forall a \in A: a * 1=1 * a=a ;
$$

4. A operação $*$ é distributiva sobre + , i.e., para qualquer $a, b, c \in A$,

$$
a *(b+c)=(a * b)+(a * c) \text { e }(b+c) * a=(b * a)+(c * a)
$$

Dizemos que $A$ é comutativo se $\forall a, b \in A: a * b=b * a$.

\section{Corpo}

Um corpo $\mathcal{K}$ é um anel comutativo no qual todos os elementos não nulos possuem inverso multiplicativo, ou seja:

$$
\forall a \in \mathcal{K}, a \neq 0 \Rightarrow \exists b \in \mathcal{K} \mid a b=1 .
$$

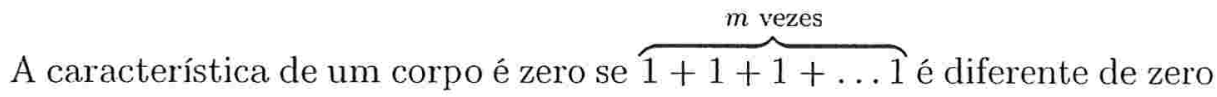
para qualquer $m>1$. Caso contrário, a característica do corpo é o menor $m$ para o qual esta soma é zero.

Se o número de elementos do corpo é finito, ele é chamado de corpo finito (finite field).

A ordem de um corpo finito é o número de elementos no corpo. Existe um corpo finito $\mathbb{F}_{q}$ de ordem $q$ se e somente se $q$ é uma potência prima, isto é, $q=p^{m}$, onde $p$ é um número primo denominado característica de $\mathbb{F}_{q}$, e $m$ é um inteiro positivo. Se $p=2$ então $\mathbb{F}$ é conhecido como corpo binário, ou corpo de característica 2. Se $p>2$ e $m=1$ então $\mathbb{F}_{q}$ é conhecido como um corpo primo ou corpo de característica prima $p$. Se $p \geq 2$ e $m \geq 2$, então $\mathbb{F}_{q}$ é um corpo estendido de característica $p$.

\section{Corpo primo}

Seja $p>2$ e $m=1$. Os inteiros módulo $p$, consistindo dos inteiros $\{0,1,2, \ldots, p-$ $1\}$ com adição e subtração módulo $p$, é um corpo finito de ordem $p$. Denominamos este corpo por $\mathbb{F}_{p}$.

Exemplo (corpo $\mathbb{F}_{29}$ ). Os elementos de $\mathbb{F}_{29}$ são $\{0,1,2, \ldots, 28\}$. Alguns exemplos de operações aritméticas em $\mathbb{F}_{29}$ são $\{0,1,2, \ldots, 28\}$. Alguns exemplos de operações aritméticas em $\mathbb{F}_{29}$ são:

Adição: $17+20=8$ desde que $37 \bmod 29=8$

Subtração: $17-20=26$ desde que $-3 \bmod 29=26$.

Multiplicação: $17 \cdot 20=21$ desde $340 \bmod 29=21$

Inversão: $17^{-1}=12$ desde $17 \cdot 12 \bmod 29=1$. 


\section{Corpo binário}

Corpos finitos de ordem $2^{m}$ são chamados de corpos binários, corpos de característica 2 ou Corpo Finito de Galois. Uma maneira de construir $\mathbb{F}_{2^{m}}$ é usar a representação na base polinomial. Nesta representação os elementos de $\mathbb{F}_{2^{m}}$ são polinômios binários (polinômios cujos coeficientes estão no corpo $\left.\mathbb{F}_{2}=\{0,1\}\right)$ de grau $\leq m-1$ :

$$
\mathbb{F}_{2^{m}}=\left\{a_{m-1} x^{m-1}+a_{m-2} x^{m-2}+\cdots+a_{2} x^{2}+a_{1} x^{1}+a_{0}: a_{i} \in\{0,1\}\right\}
$$

Um polinômio binário irredutível $f(x)$ de grau $m$ é escolhido (tal polinômio existe para $m$ e pode ser eficientemente computado). A irredutividade de $f(x)$ significa que $f(x)$ não pode ser fatorado como um produto de polinômios binários de grau menor do que $m$. A adição de elementos do corpo é a adição usual de polinômios, com os coeficientes reduzidos a módulo 2. A multiplicação de elementos do corpo é feita pela redução polinomial $f(x)$. Exemplo $\left(\mathbb{F}_{2^{4}}\right)$. Neste caso o polinômio irredutível é $f(x)=x^{4}+x+1$. Vamos representar o polinômio $b_{3} x^{3}+b_{2} x^{2}+b_{1} x^{1}+b_{0}$ pelo vetor $\left(b_{3}, b_{2}, b_{1}, b_{0}\right)$. Por exemplo:

$$
\begin{aligned}
& (1011) \oplus(1001)=(0010) \\
& (1101) \otimes(1001)=(1111),
\end{aligned}
$$

pois $\left(x^{3}+x^{2}+1\right)\left(x^{3}+1\right)=x^{6}+x^{5}+x^{2}+1=\left(x^{3}+x^{2}+x+1\right) \bmod f(x)$.

$\mathbb{F}_{2^{4}}-\{0\}$ forma um grupo multiplicativo de ordem 15: $g=(0010)$ é o gerador deste grupo: $g^{1}=(0010), g^{2}=(0100), g^{3}=(1000), g^{4}=$ (0011), $g^{5}=(0110), g^{6}=(1100), g^{7}=(1011), g^{8}=(0101), g^{9}=(1010)$, $g^{10}=(0111), g^{11}=(1110), g^{12}=(1111), g^{13}=(1101), g^{14}=(1001)$, $g^{15}=(0001)$.

\section{Corpo estendido}

A representação em base polinomial para corpos binários pode ser generalizada para todos os corpos estendidos, como se segue. Seja $p \geq 2$ um primo e $m \geq 2$. Se $\mathbb{F}_{p}[x]$ denota o conjunto de todos os polinômios na variável $x$ com coeficientes em $\mathbb{F}_{p}$. Seja $f(x)$, o polinômio redutor de grau $m$ em $\mathbb{F}_{p}[x]$. Os elementos de $\mathbb{F}_{p^{m}}$ são polinômios em $\mathbb{F}_{p}[x]$ de grau $\leq m-1$ :

$$
\mathbb{F}_{p^{m}}=\left\{a_{m-1} x^{m-1}+a_{m-2} x^{m-2}+\cdots+a_{2} x^{2}+a_{1} x^{1}+a_{0}: a_{i} \in \mathbb{F}_{p}\right\}
$$

A adição de elementos do corpo é a adição usual de polinômios, com coeficientes em $\mathbb{F}_{p}$. A multiplicação de elementos do corpo é feita pela redução polinomial $f(x)$. 


\section{$14 \mathbb{Z}, \mathbb{Z}_{n}$ e $\mathbb{Z}_{n}^{*}$}

$\mathbb{Z}$ é o conjunto de todos os inteiros com operação de soma e multiplicação usuais.

$\mathbb{Z}_{n}$ é o conjunto de todos os inteiros modn com operações de soma e multiplicação modn. Exemplo: $Z_{11}=\{0,1, \ldots, 10\}$.

$\mathbb{Z}_{n}^{*}$ é o conjunto de todos os inteiros modn relativamente primos a $n$. Exemplo $\mathbb{Z}_{10}^{*}=\{1,3,7,9\}$.

$\mathbb{Z}_{n}$ é um anel. Se $n$ é primo, então $\mathbb{Z}_{n}$ é um corpo.

\section{Bases normais de $\mathbb{F}_{2^{m}}$}

O corpo $\mathbb{F}_{2^{m}}$ pode ser visto como um espaço vetorial de dimensão $m$ sobre $\mathbb{F}_{2}$. Existem muitas bases para esse espaço vetorial, e há uma representação dos elementos do corpo para cada base adotada. Algumas bases podem propiciar implementações das operações de forma mais eficiente do que outras. A dificuldade computacional do problema do logaritmo discreto sobre $\mathbb{F}_{2^{m}}$ não depende da base adotada.

Uma base normal para $\mathbb{F}_{2^{m}}$ sobre $\mathbb{Z}_{2}$ é uma base da forma

$$
\left\{\beta, \beta^{2}, \beta^{2^{2}}, \ldots, \beta^{2^{m}-1}\right\}
$$

onde $\beta \in \mathbb{F}_{2^{m}}$. Notamos que $\beta^{2 m}=\beta$.

Seja $\alpha=\sum_{j=0}^{m-1} b_{j} \beta^{2^{j}} \in \mathbb{F}_{2^{m}}$. A representação vetorial de $\alpha$ é

$$
\left(b_{0}, b_{1}, \ldots, b_{m-1}\right) \text {. }
$$

Como a elevação ao quadrado é linear em $\mathbb{F}_{2^{m}}$, tem-se que

$$
\alpha^{2}=\sum_{j=0}^{m-1} b_{j} \beta^{2^{j+1}}=\sum_{j=0}^{m-1} b_{j-1} \beta^{2^{j}}
$$

ou seja, a elevação ao quadrado é um simples deslocamento circular de uma posição para a direita .

Exemplo. Vamos considerar novamente $\mathbb{F}_{2^{4}}$ e $f(x)=x^{4}+x+1$. Existem duas bases normais para $\mathbb{F}_{2^{4}}$ :

$$
A=\left\{x^{3}, x^{6}, x^{12}, x^{9}\right\} \quad \text { e } \quad B=\left\{x^{7}, x^{14}, x^{13}, x^{11}\right\} .
$$

Vamos considerar um elemento $\alpha=x^{6}+x^{3}=(1,1,0,0)$ representado na base $A$ (que equivale a $\alpha=x^{2}$ na base polinomial). Então, $\alpha^{2}=x^{12}+2 x^{9}+$ $x^{6}=x^{12}+x^{6}=(0,1,1,0)$ (equivalente a $\alpha^{2}=x+1$ na base polinomial). Portanto, conforme mostrado, a elevação ao quadrado na base normal de $\alpha$ foi apenas um deslocamento circular de uma posição a direita da representação vetorial de $\alpha$. 


\section{Homomorfismo}

Um mapeamento $f: G \rightarrow H$ de um grupo $G$ em um grupo $H$ é chamado um homomorfismo se $f$ preserva as operações de $G$. Isto é, se $*$ e $\bullet$ são operações $G$ e $H$, repectivamente, então $f$ preserva as operações de $G$ se para todos $a, b \in G$ termos $f(a * b)=f(a) \bullet f(b)$.

\section{Endomorfismo}

Um homomorfismo de $G$ em $G$ é chamado de endomorfismo.

\section{Relação de equivalência}

Uma relação de equivalência é uma relação ao mesmo tempo reflexiva, simétrica e transitiva.

Seja $G$ um grupo. Uma relação de equivalência em $G$ induz a partição de $G$ em subconjuntos não-vazios mutuamente disjuntos. Esses conjuntos são denominados classes de equivalência. 


\section{Apêndice B}

\section{Implementação}

Esta implementação está dividida nos seguintes tópicos: multiplicação em curvas definidas sobre corpos binários (página 134); Multiplicação em curvas definidas sobre corpos primos (página 146) e obtenção de parâmetros com o MAGMA (página 166).

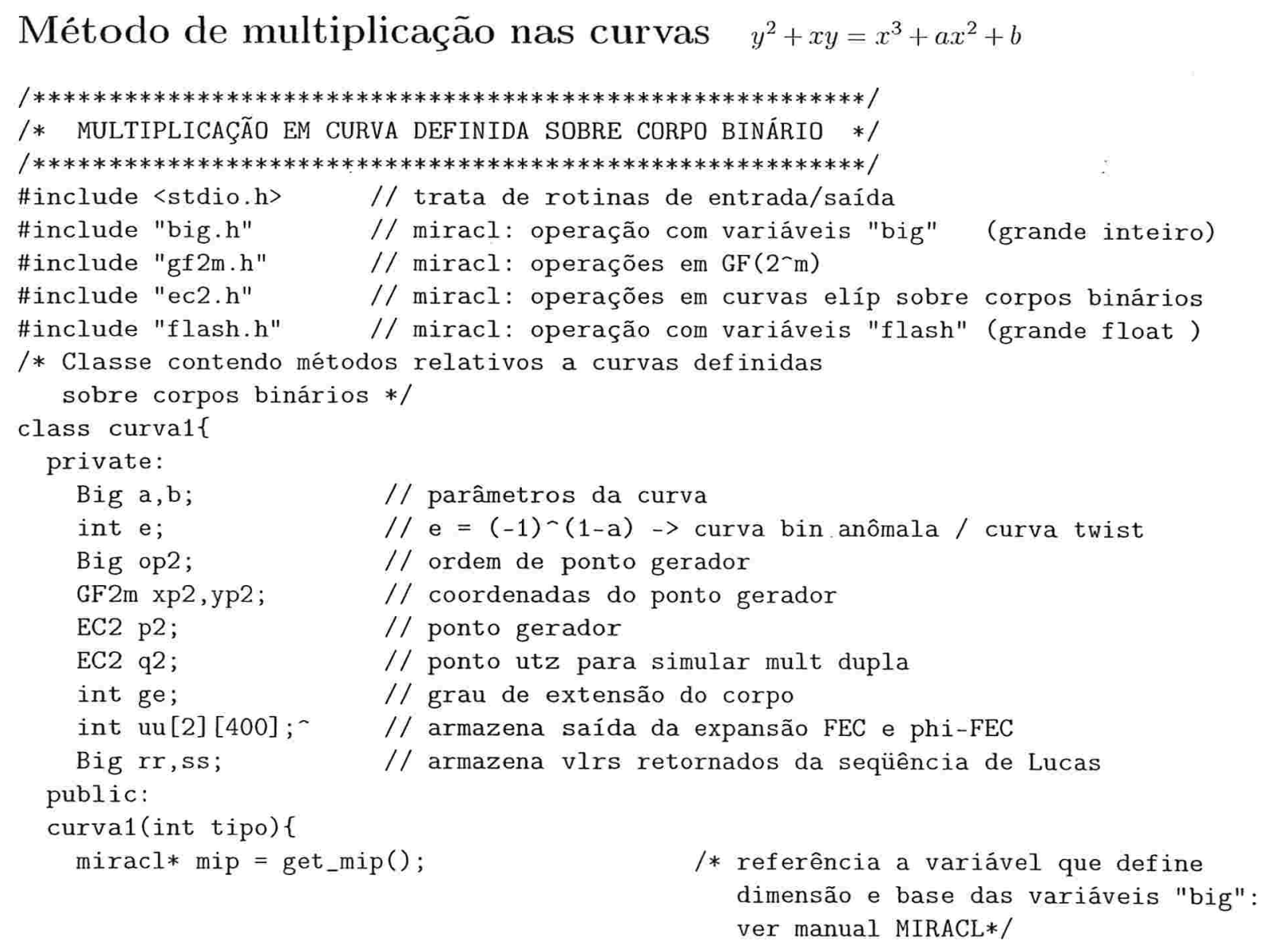




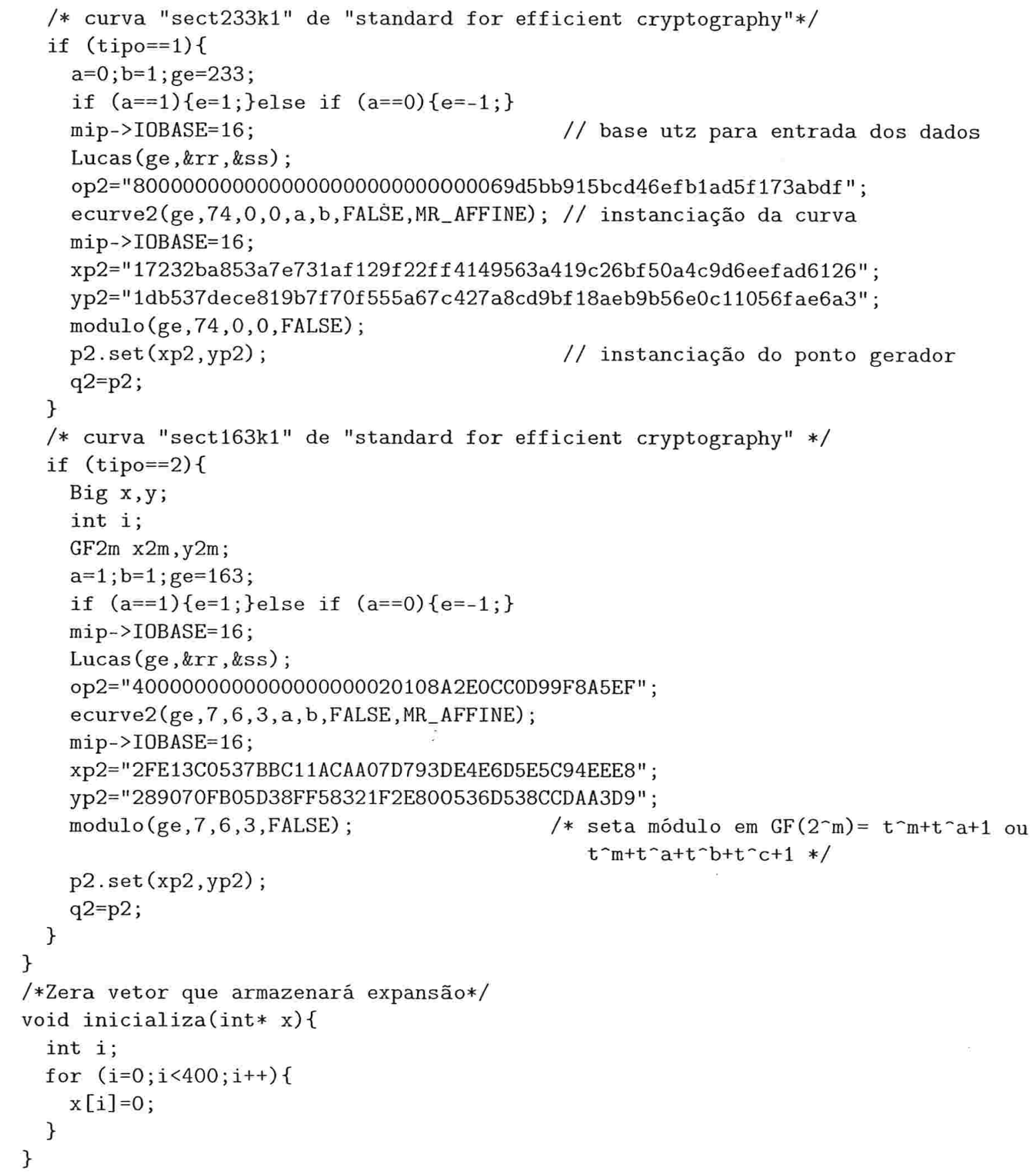

/*Sequência de Lucas (ver página 54)*/

/*n=grau de extensão do corpo da definição da curva*/

void Lucas (int $\mathrm{n}$, Big* $r$, Big* $s$ ) \{

int $i$;

Big un_1,un_2, un; 


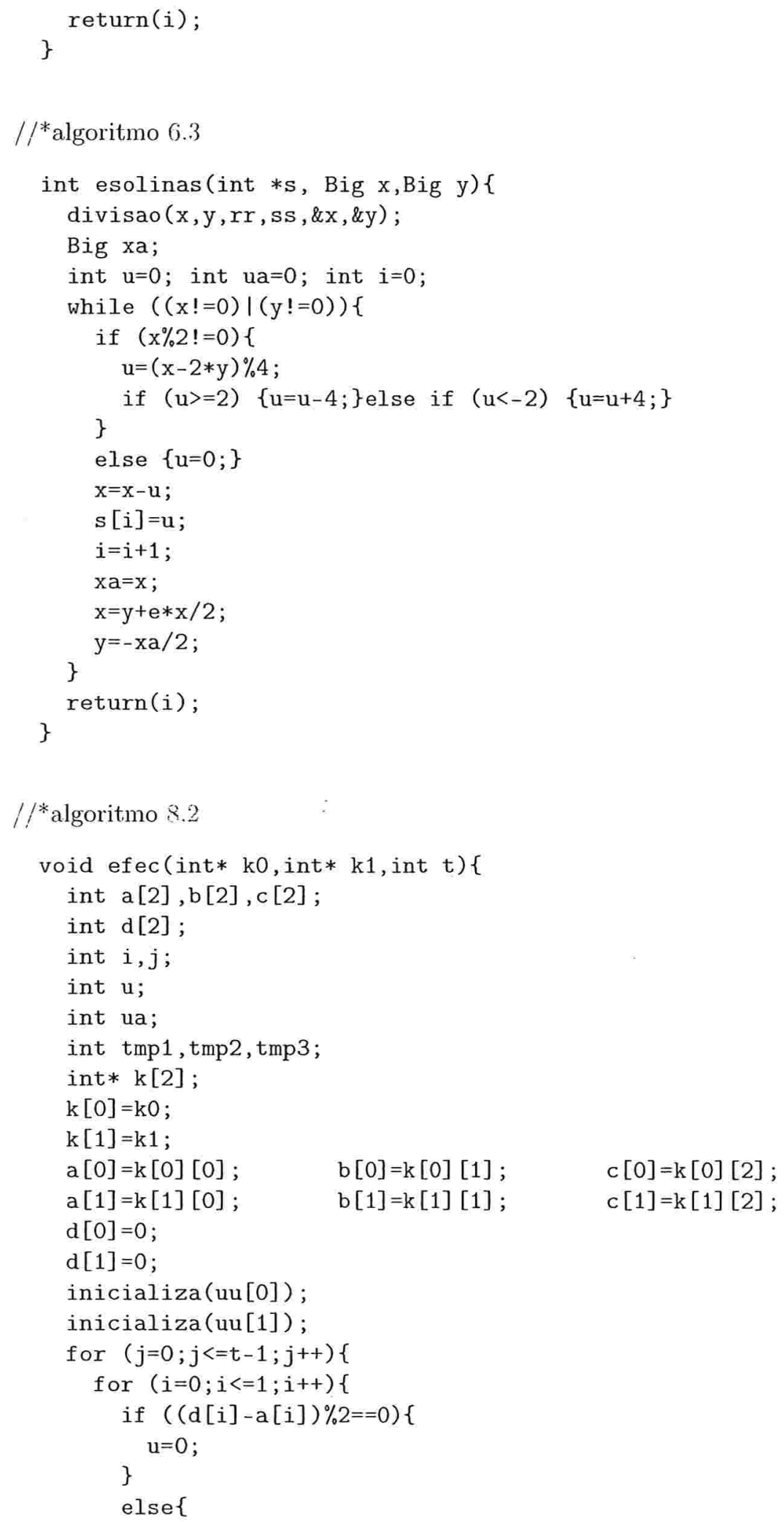




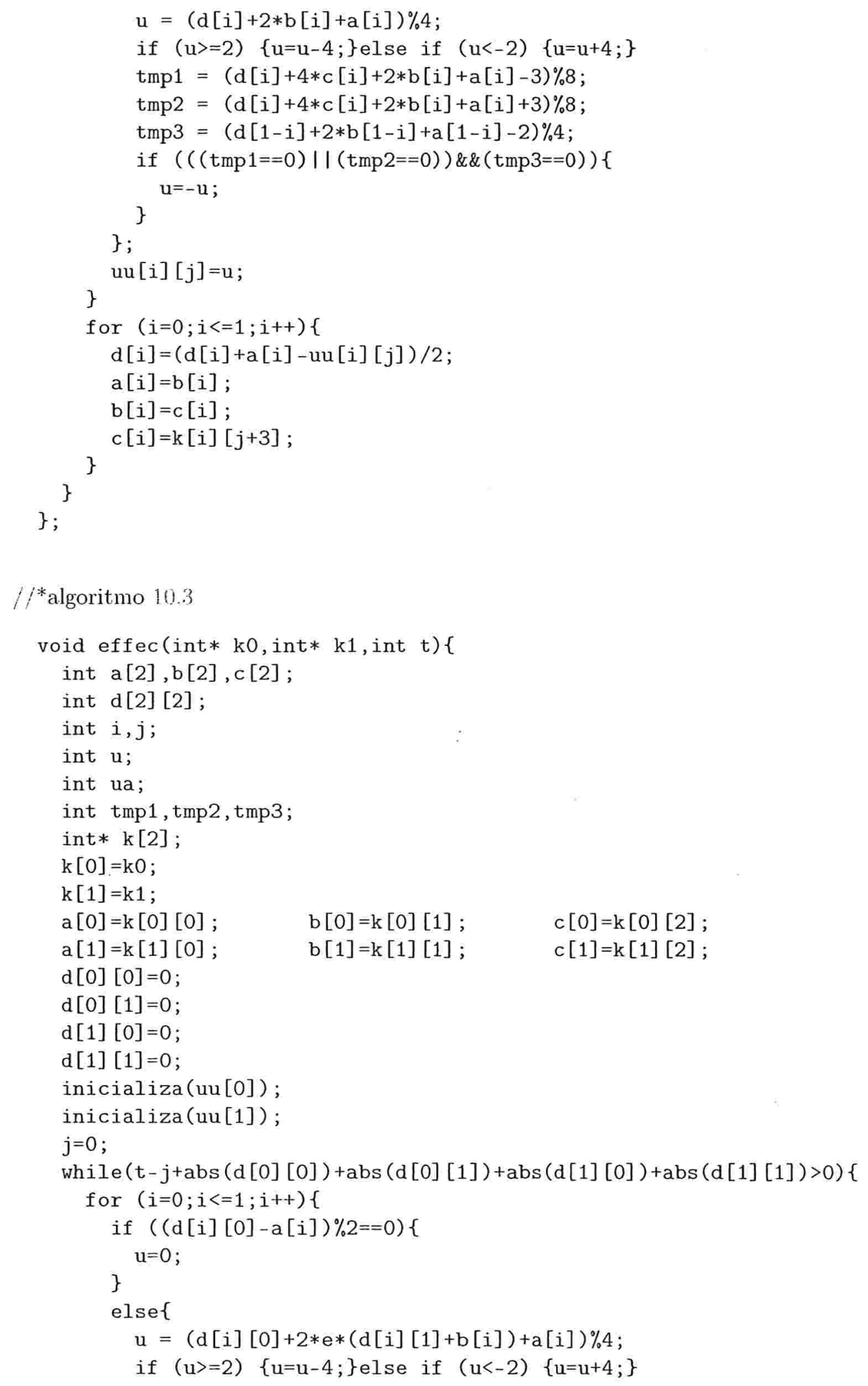




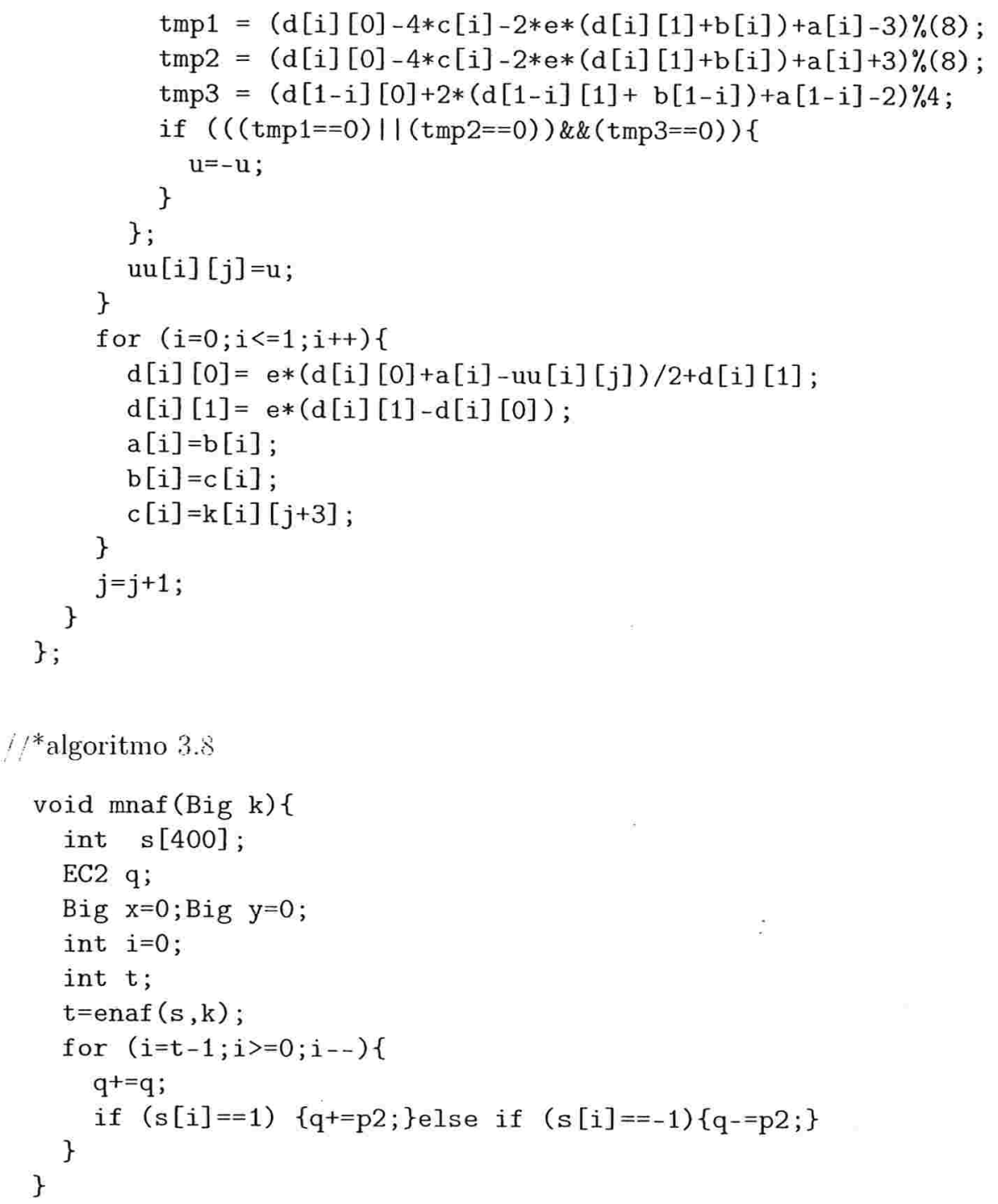




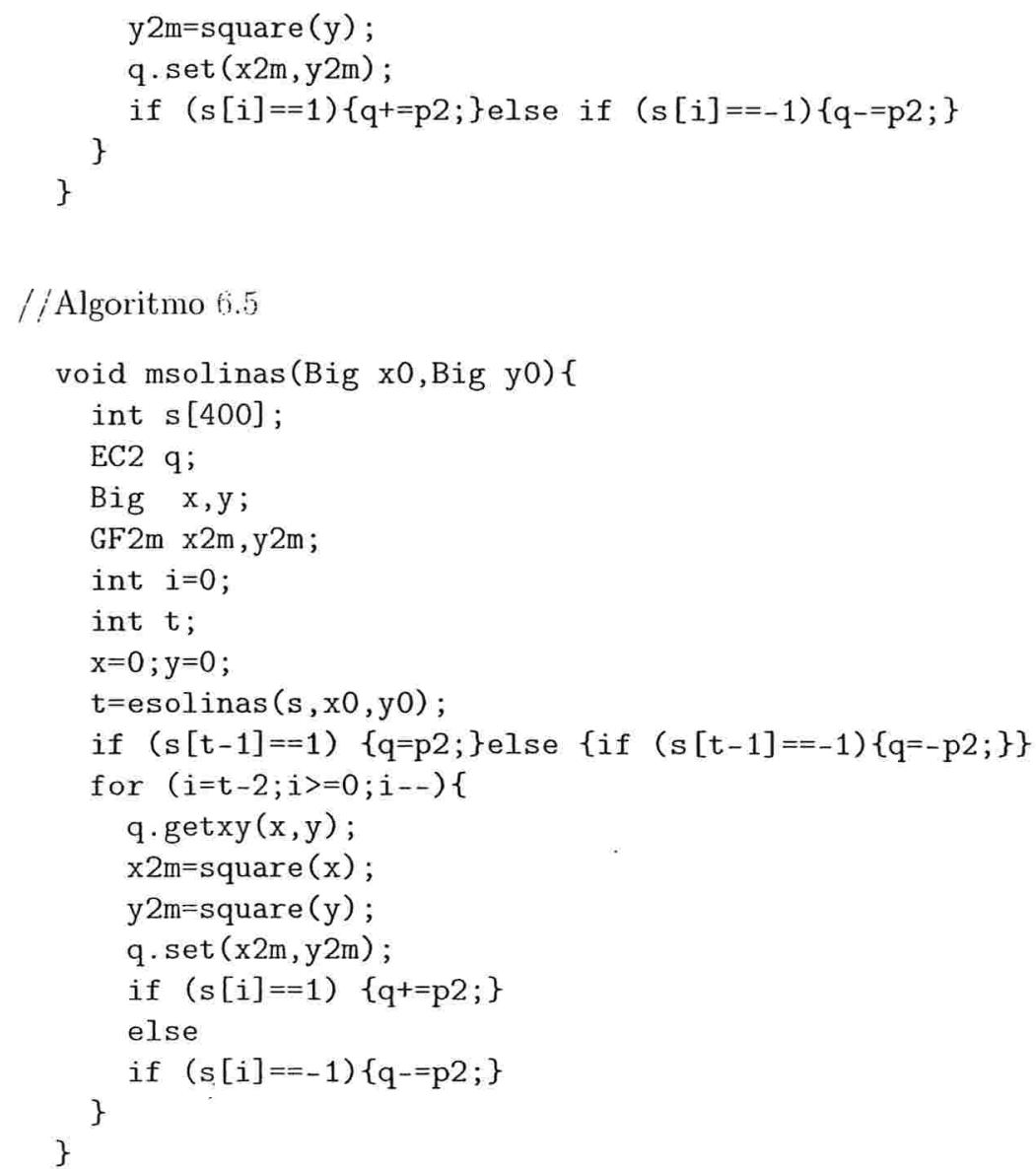




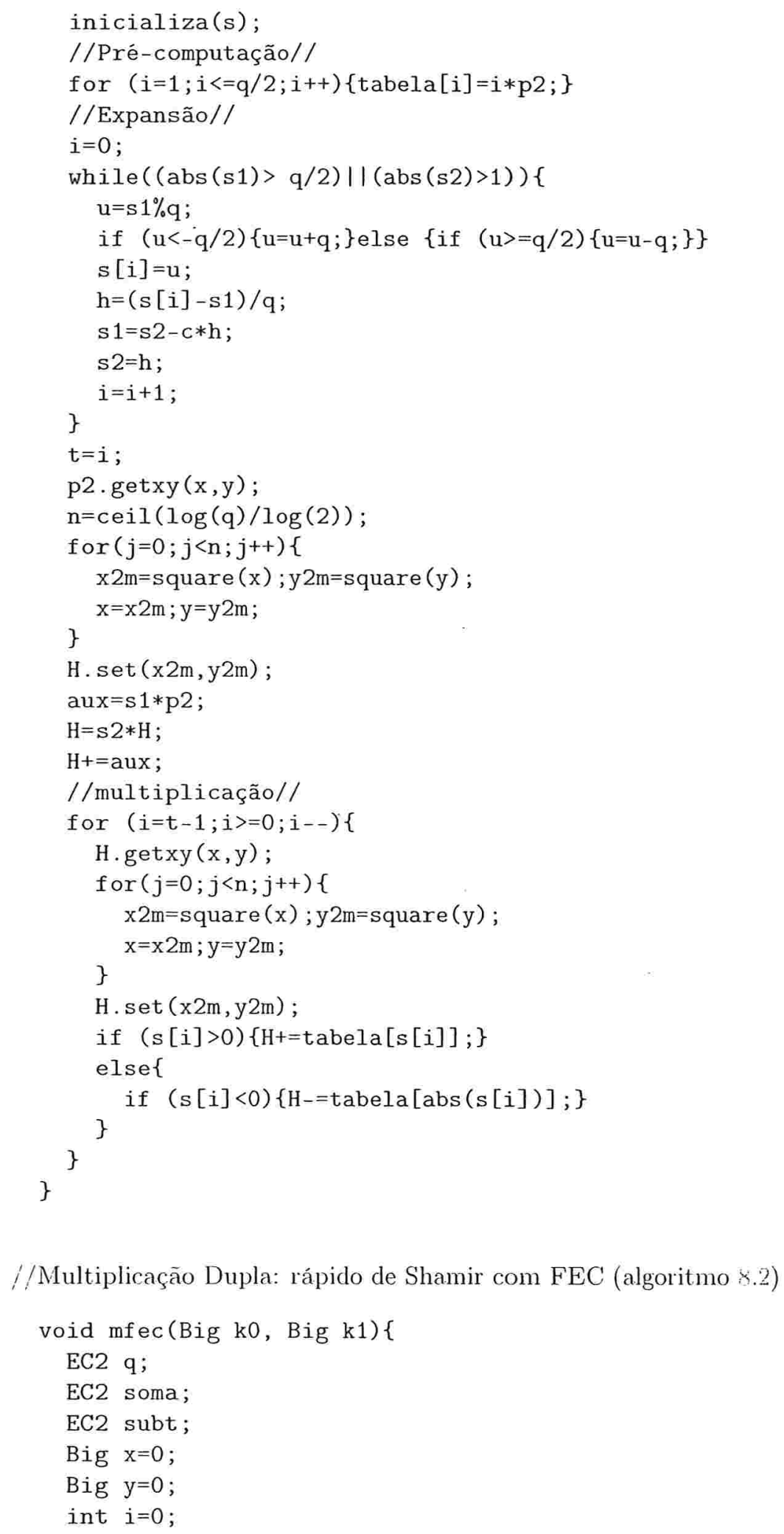

//Multiplicação Dupla: rápido de Shamir com FEC (algoritmo 8.2) 


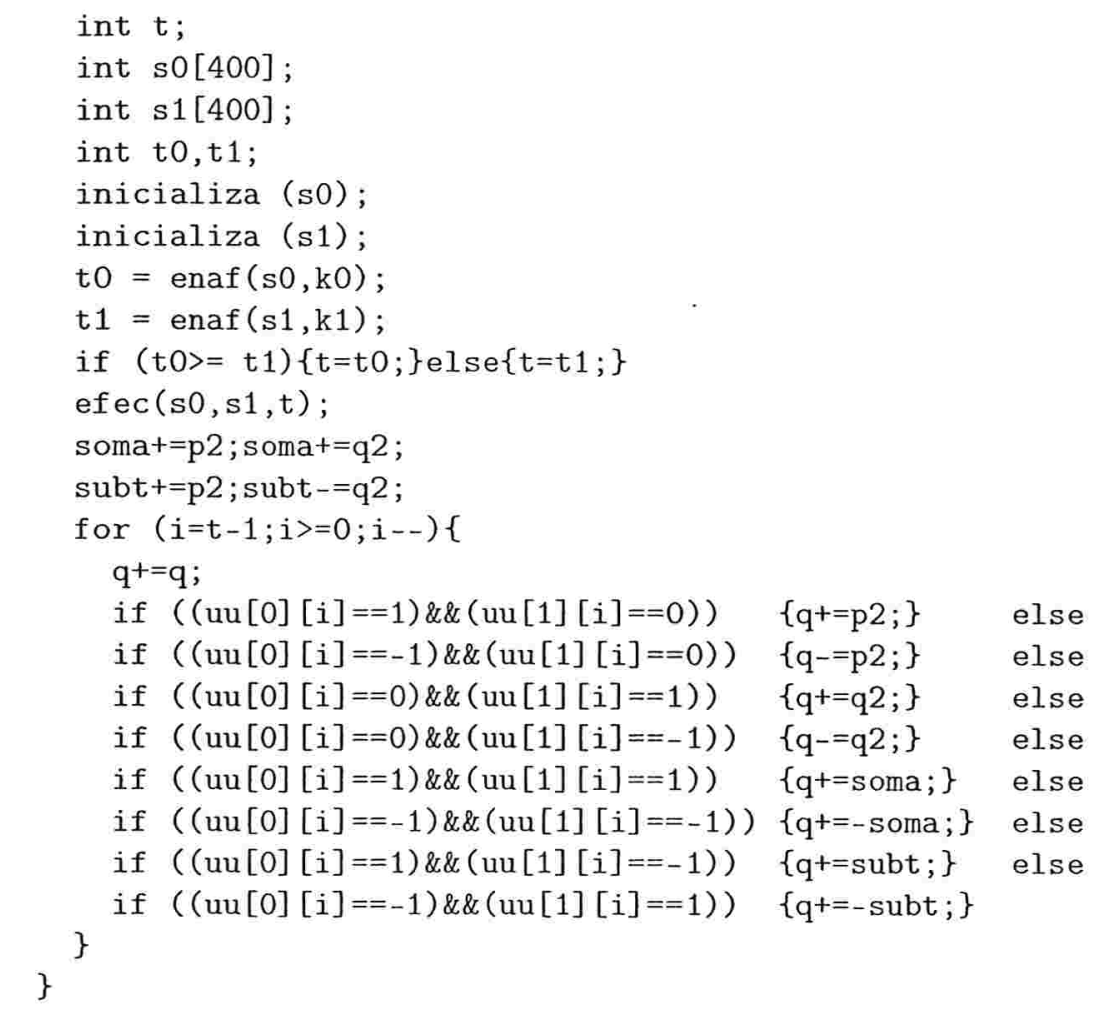

//Multiplicação Dupla: rápido de Shamir com $\phi$-FEC (algoritmo 10.3)

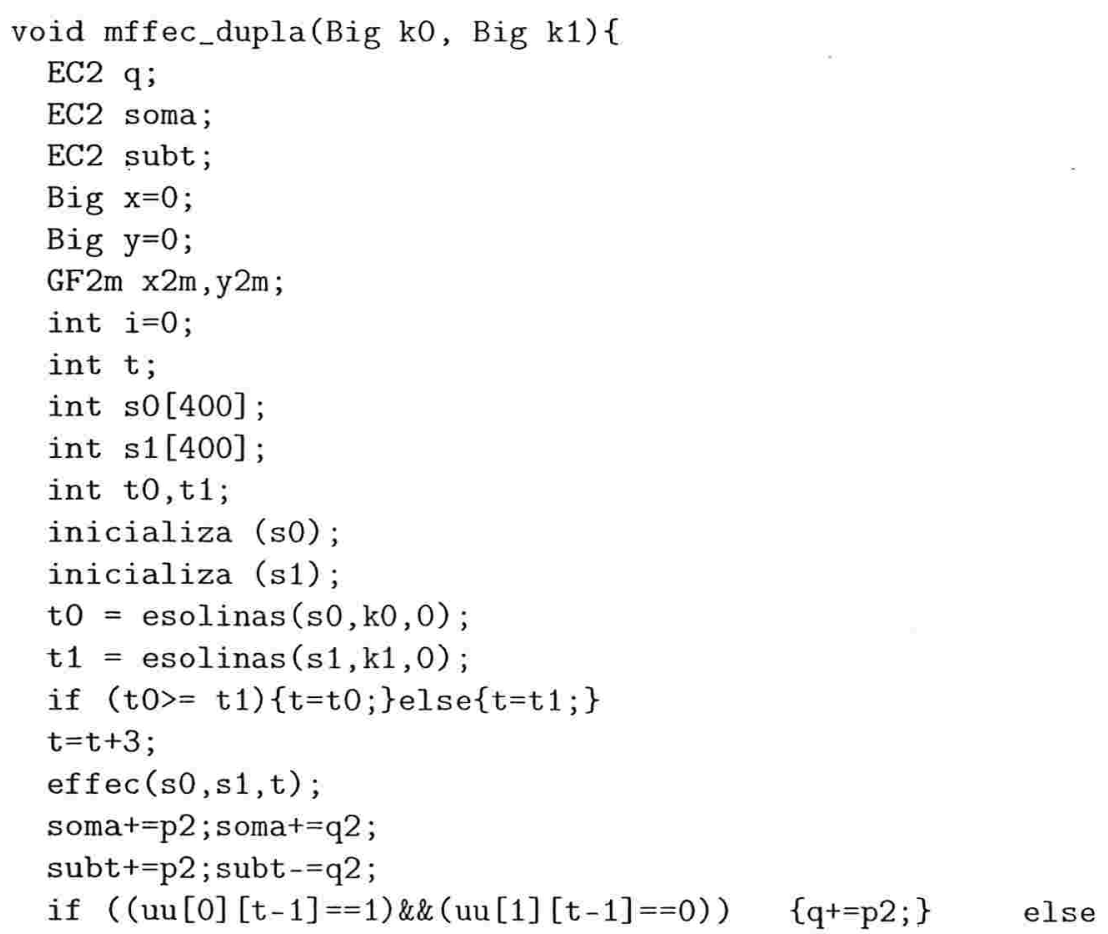




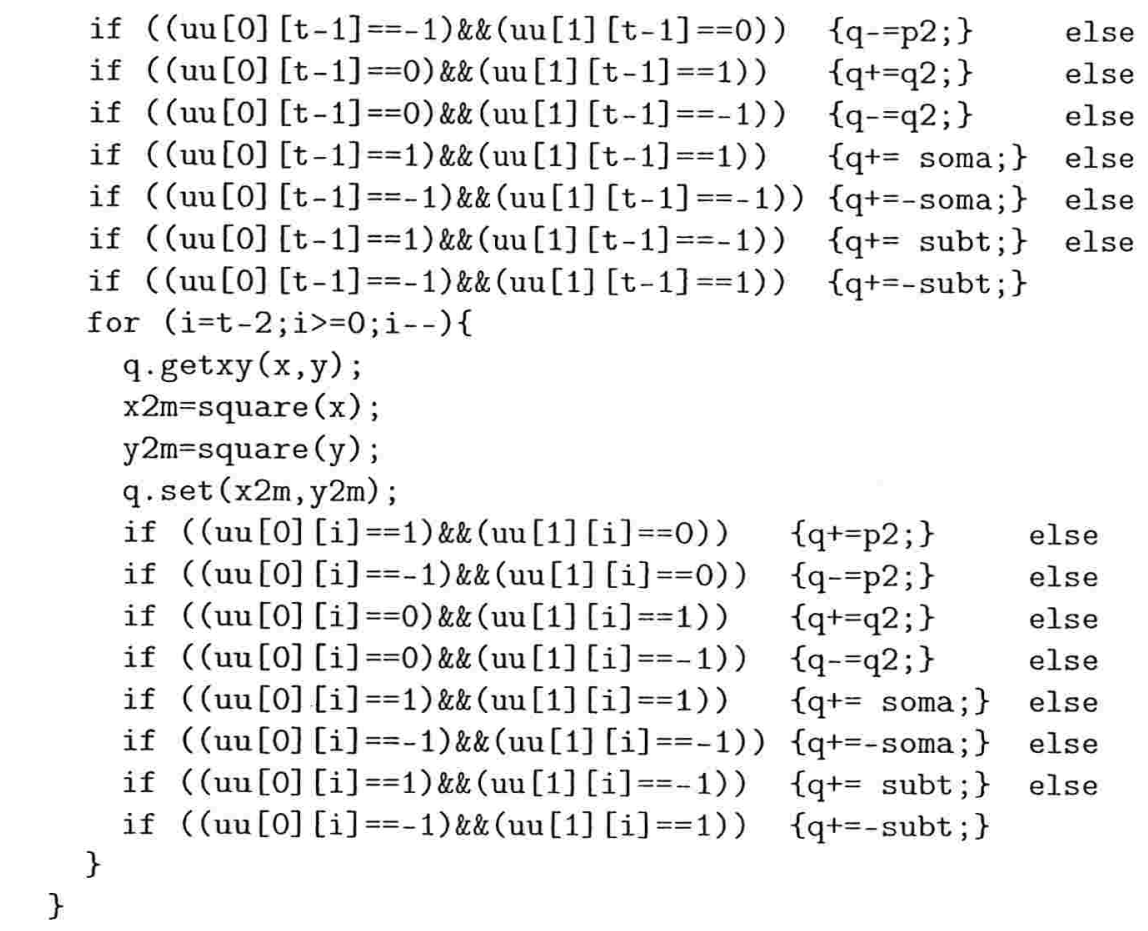

//Multiplicação Simples: $\phi$-FEC (algoritmo 10.3)

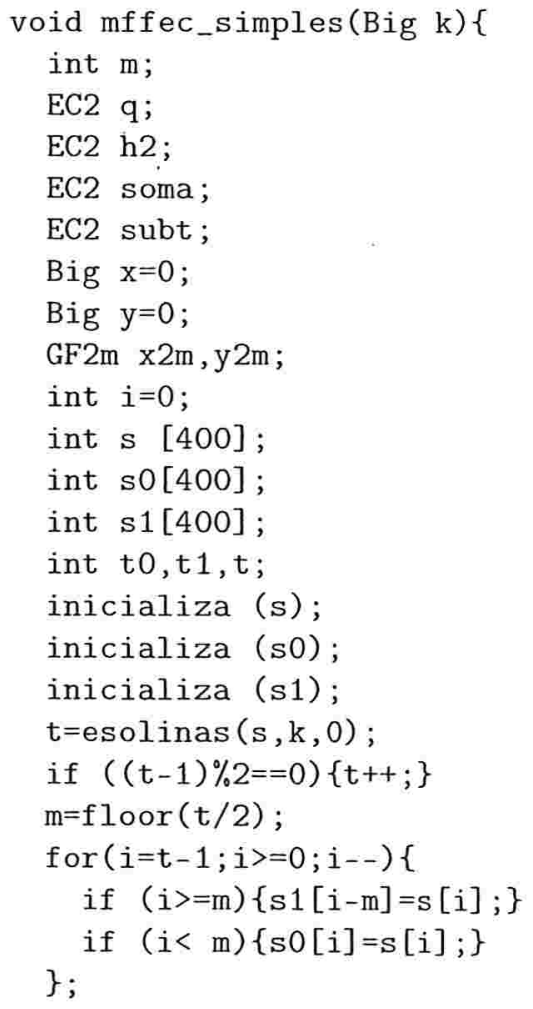




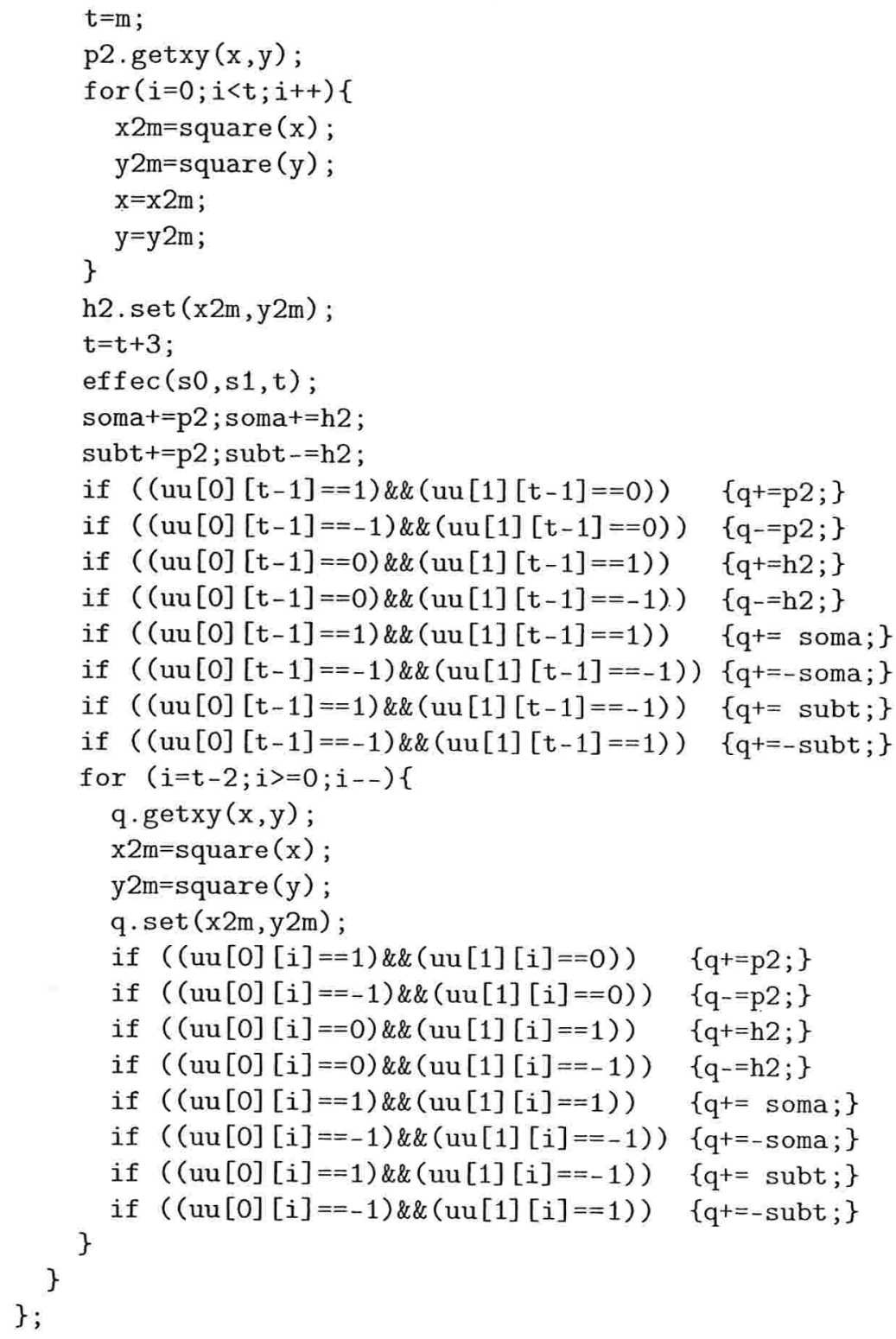




\section{Método de multiplicação nas curvas $y^{2}=x^{3}+a a x+b$}

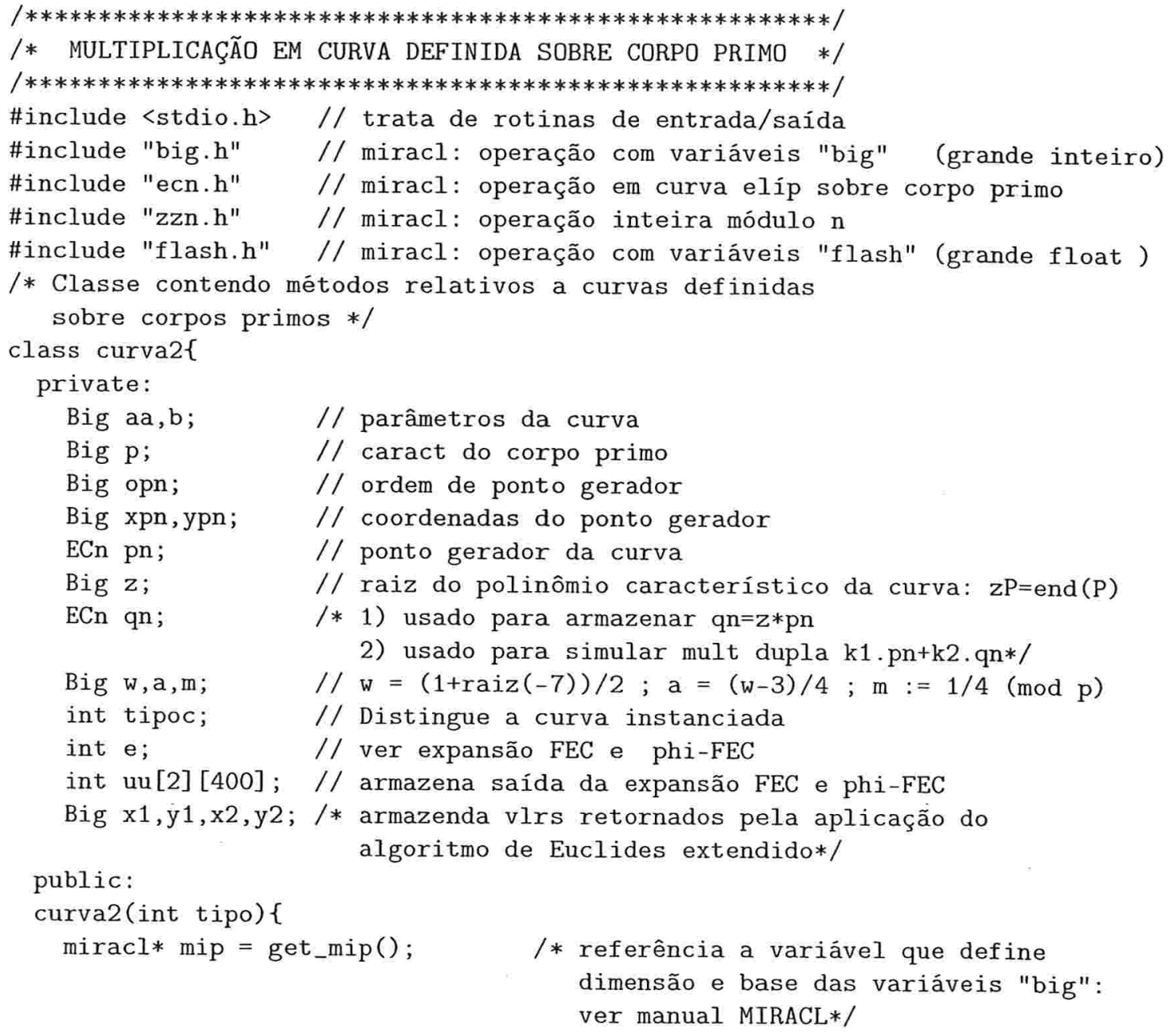

/*Curva do exemplo 10.1 -> parâmetros obtidos com o MAGMA*/

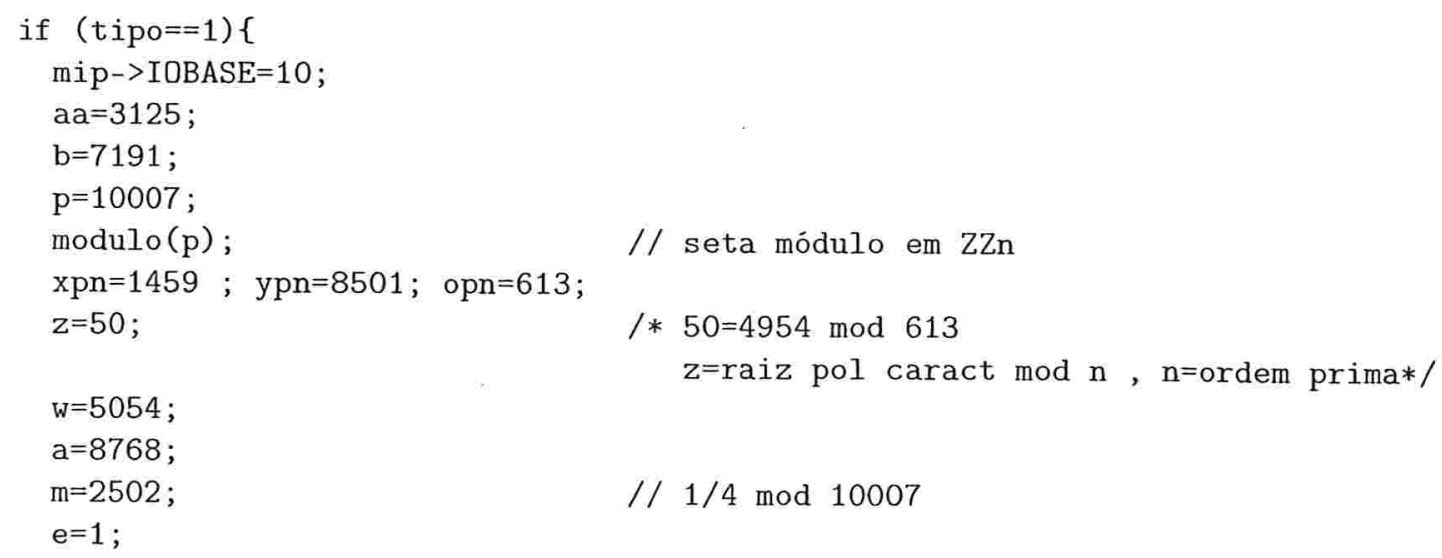




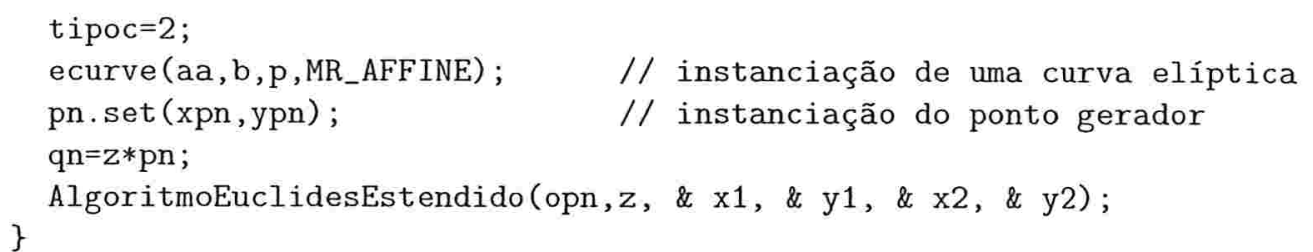

/*Curva do exemplo 10.1 -> parâmetros obtidos com o MAGMA*/

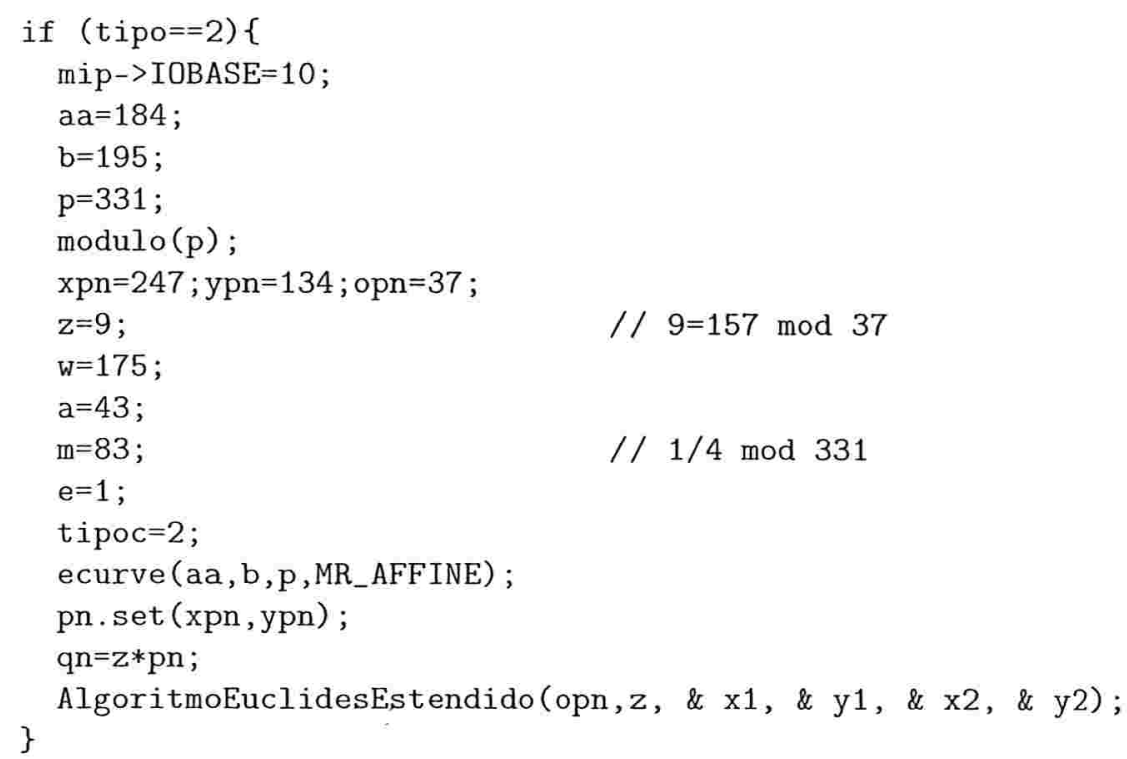

$/$ Curva do exemplo 10.2 -> parâmetros obtidos com o MAGMA*/

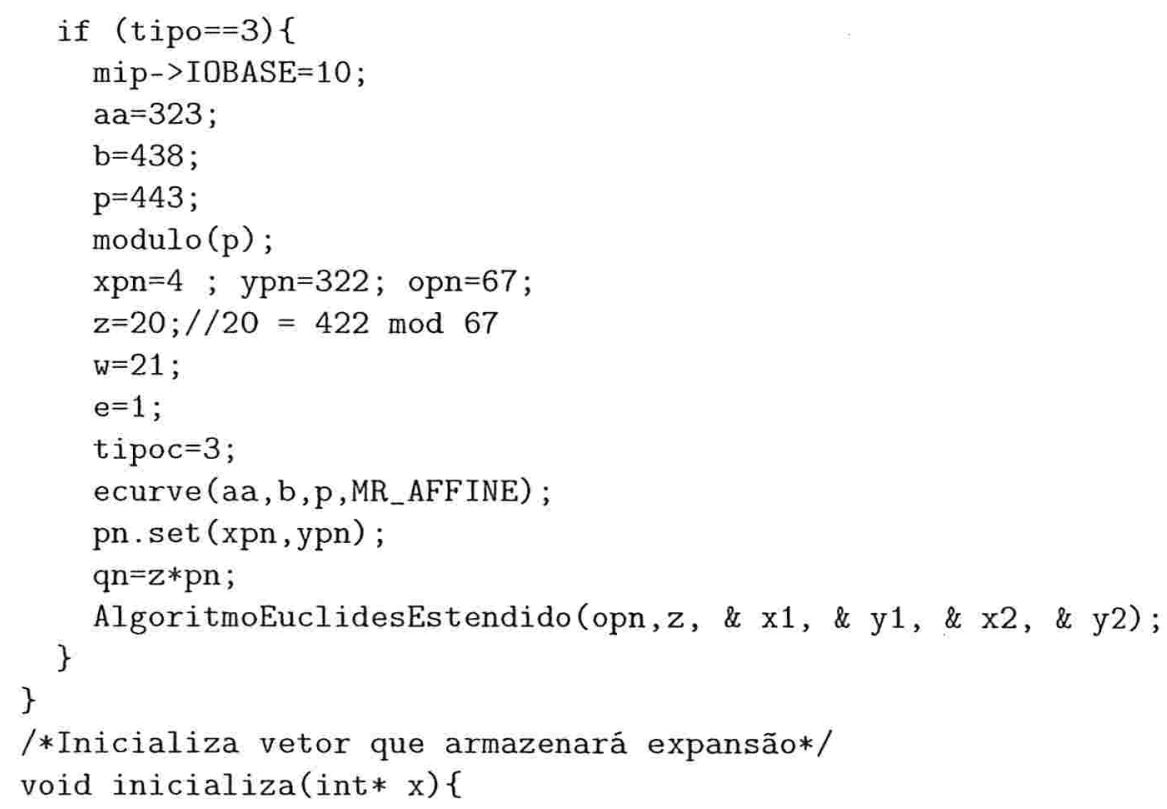




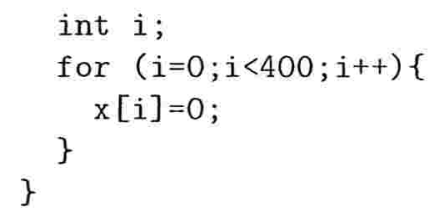

${ }^{*}$ Computação dos endomorfismos das curvas dos exemplos 10.1 e $10.2^{*} /$

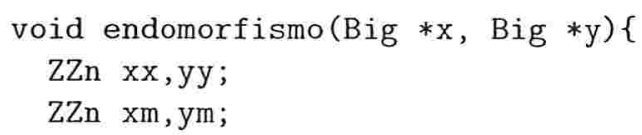

Exemplo 10.1

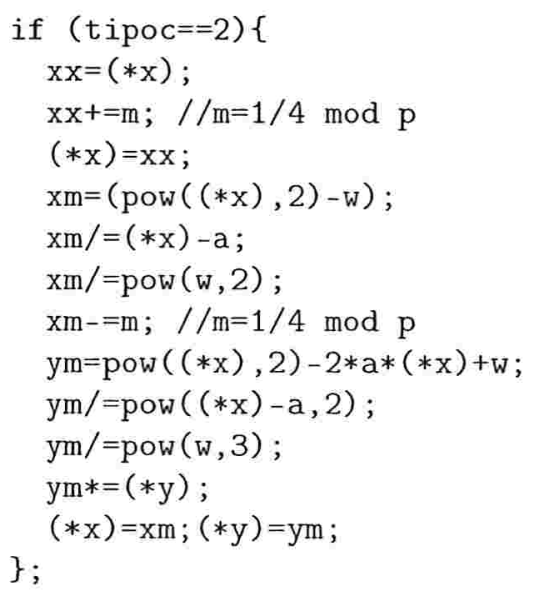

Exemplo 10.2

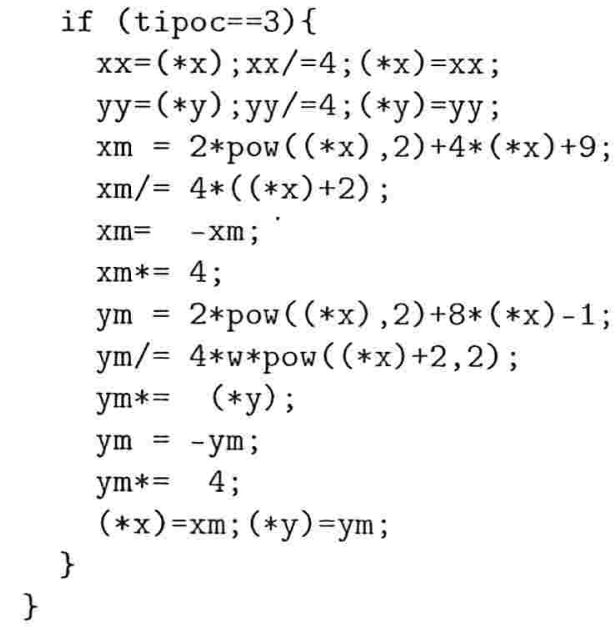

//Algoritmo 9.2 


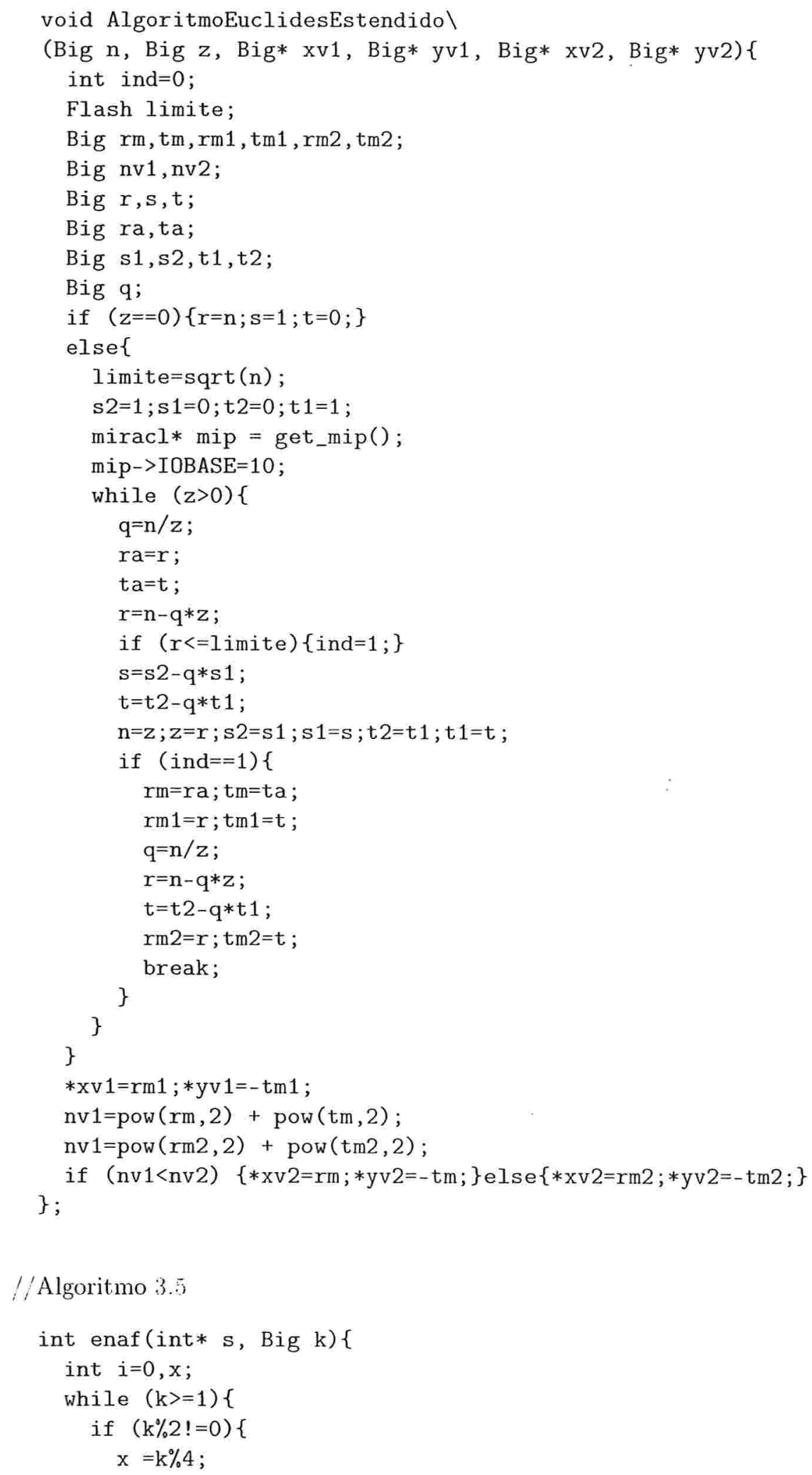




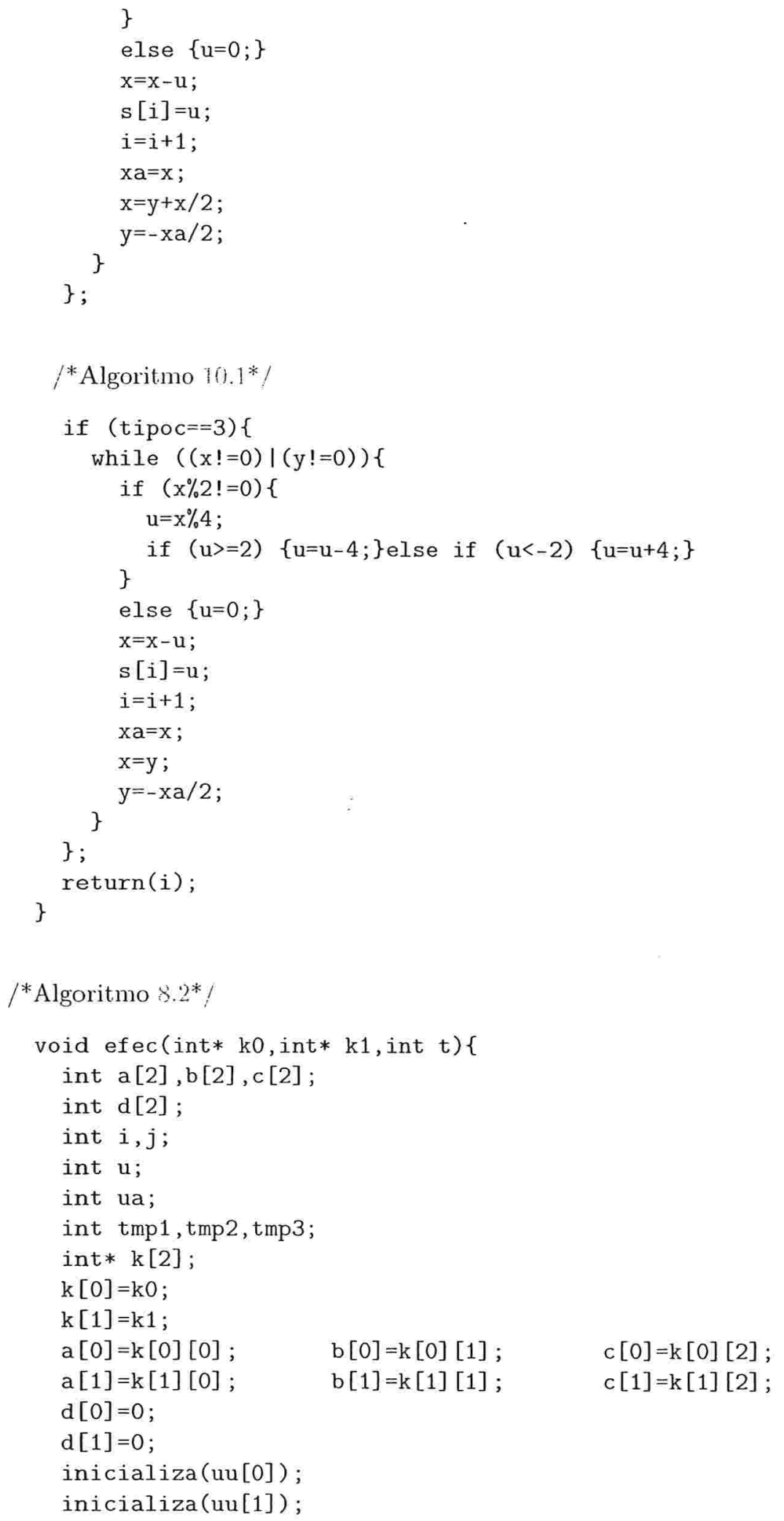




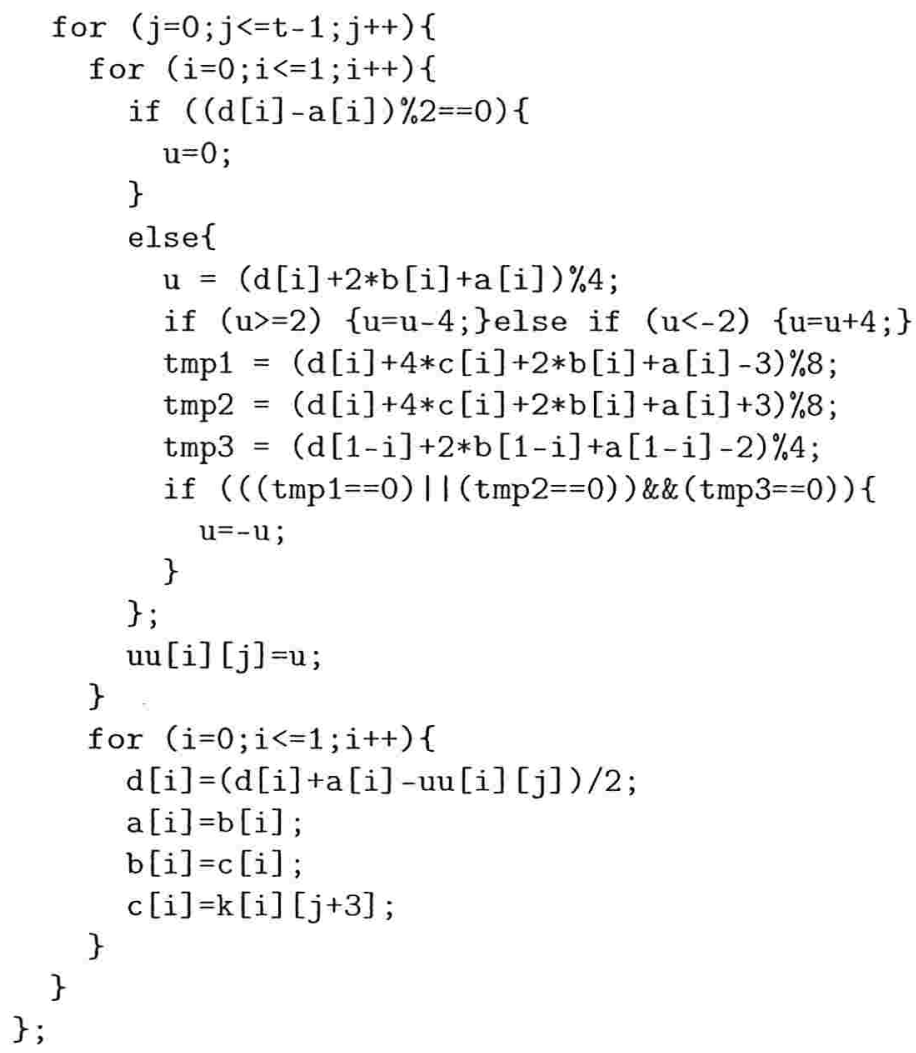




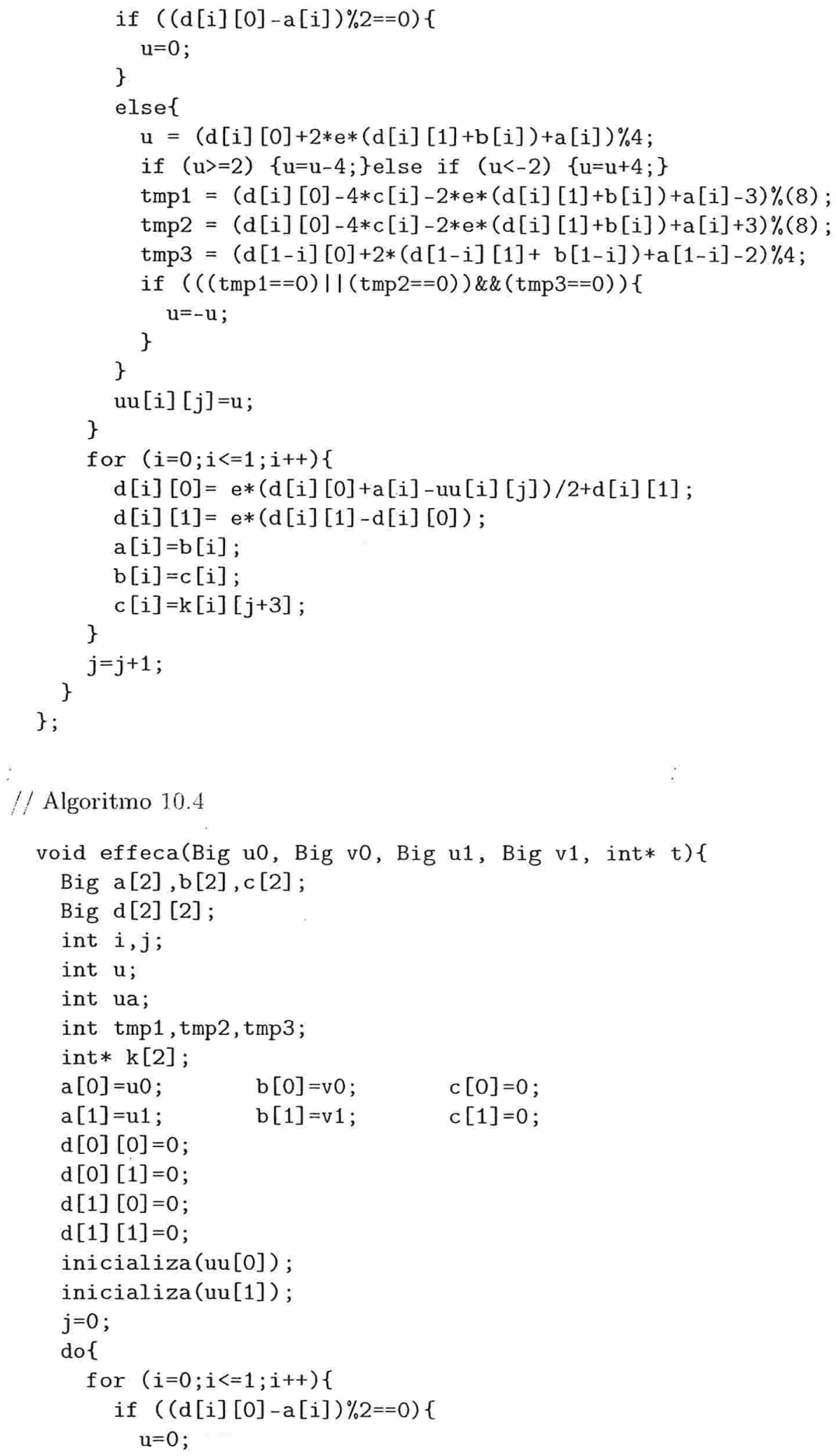




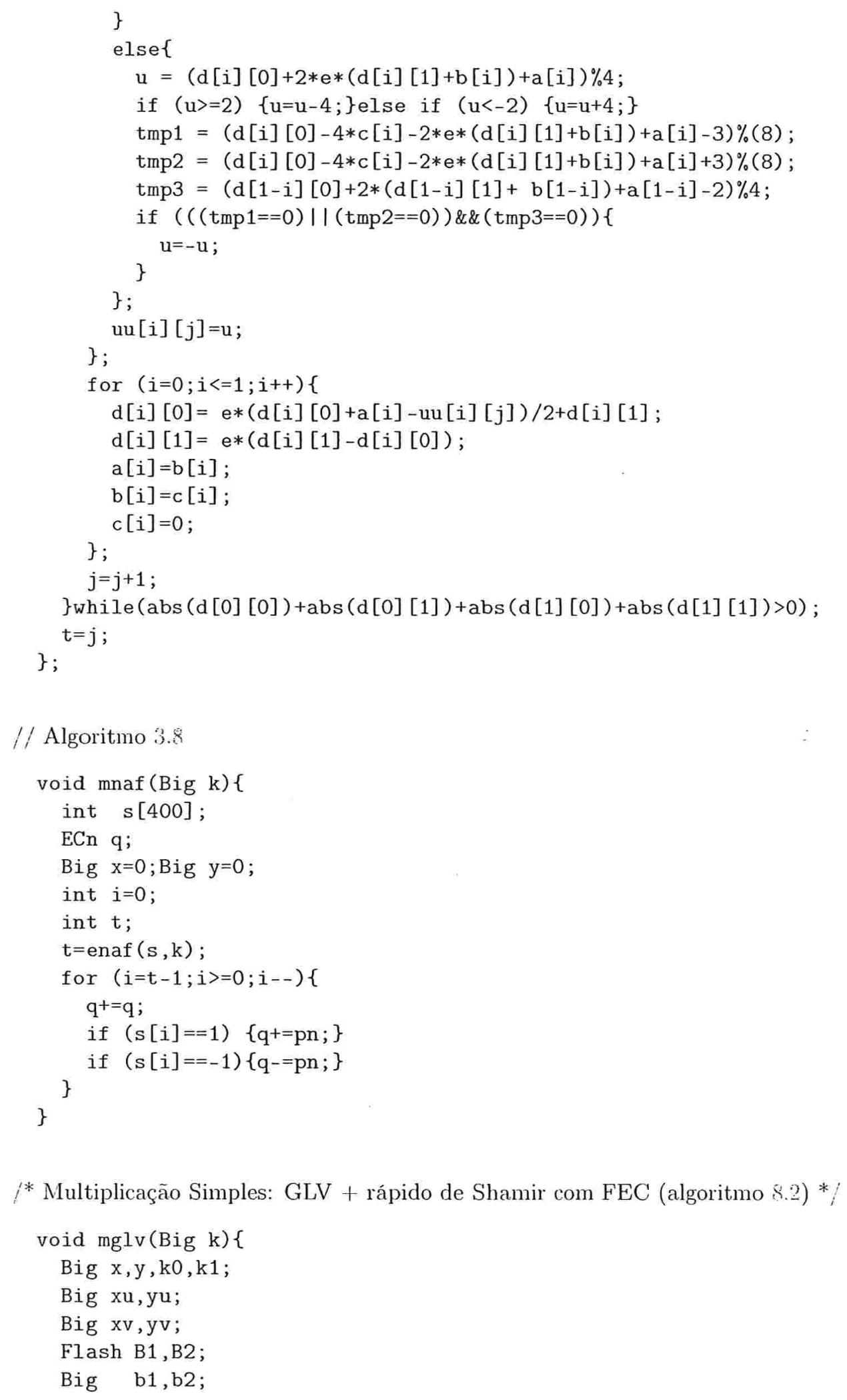




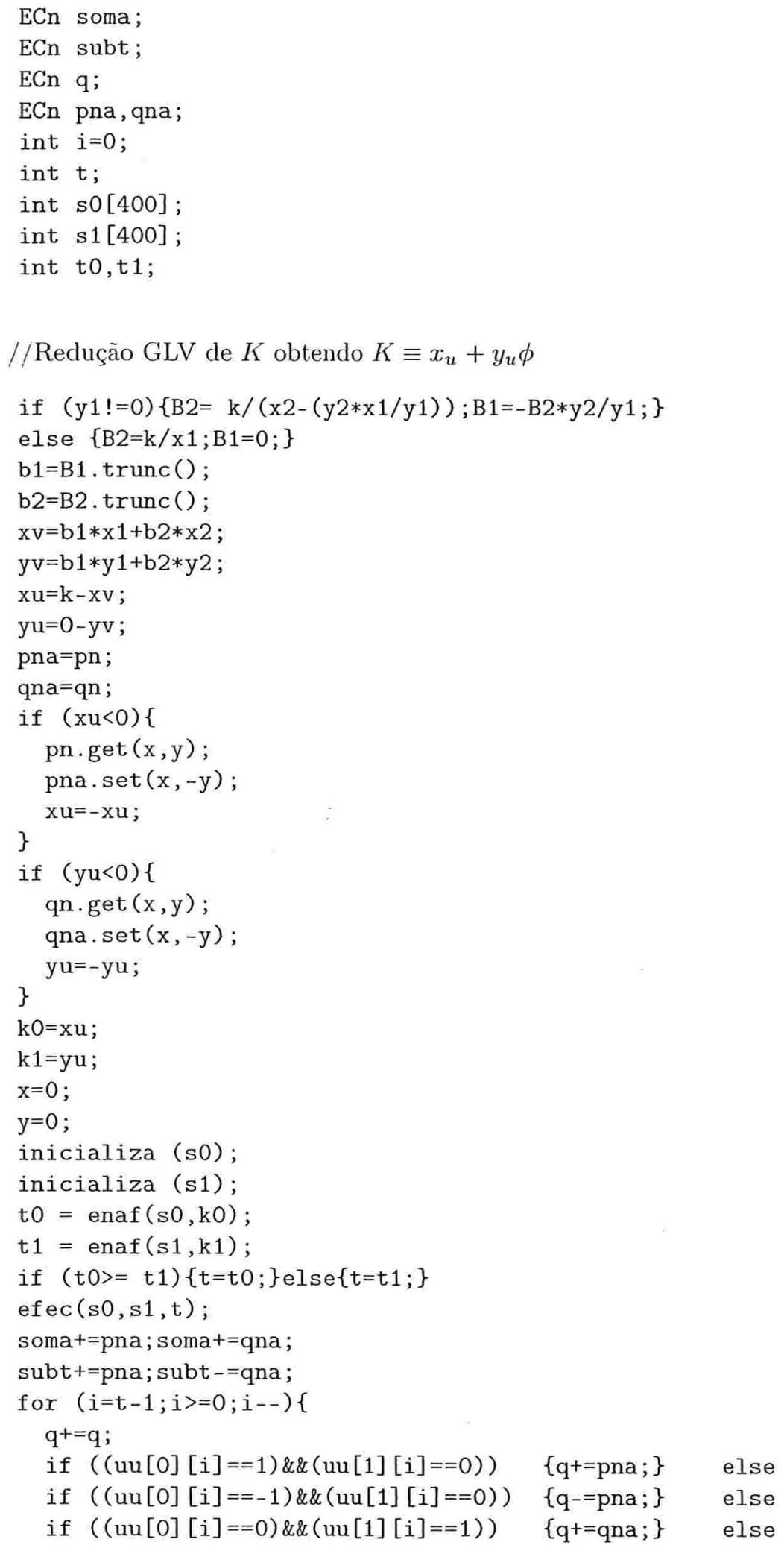




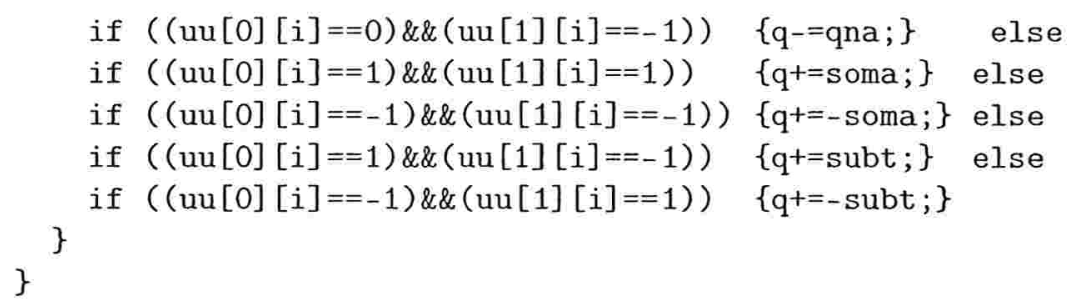

// Multiplicação Dupla: rápido de shamir com FEC (algoritmo \$.2)

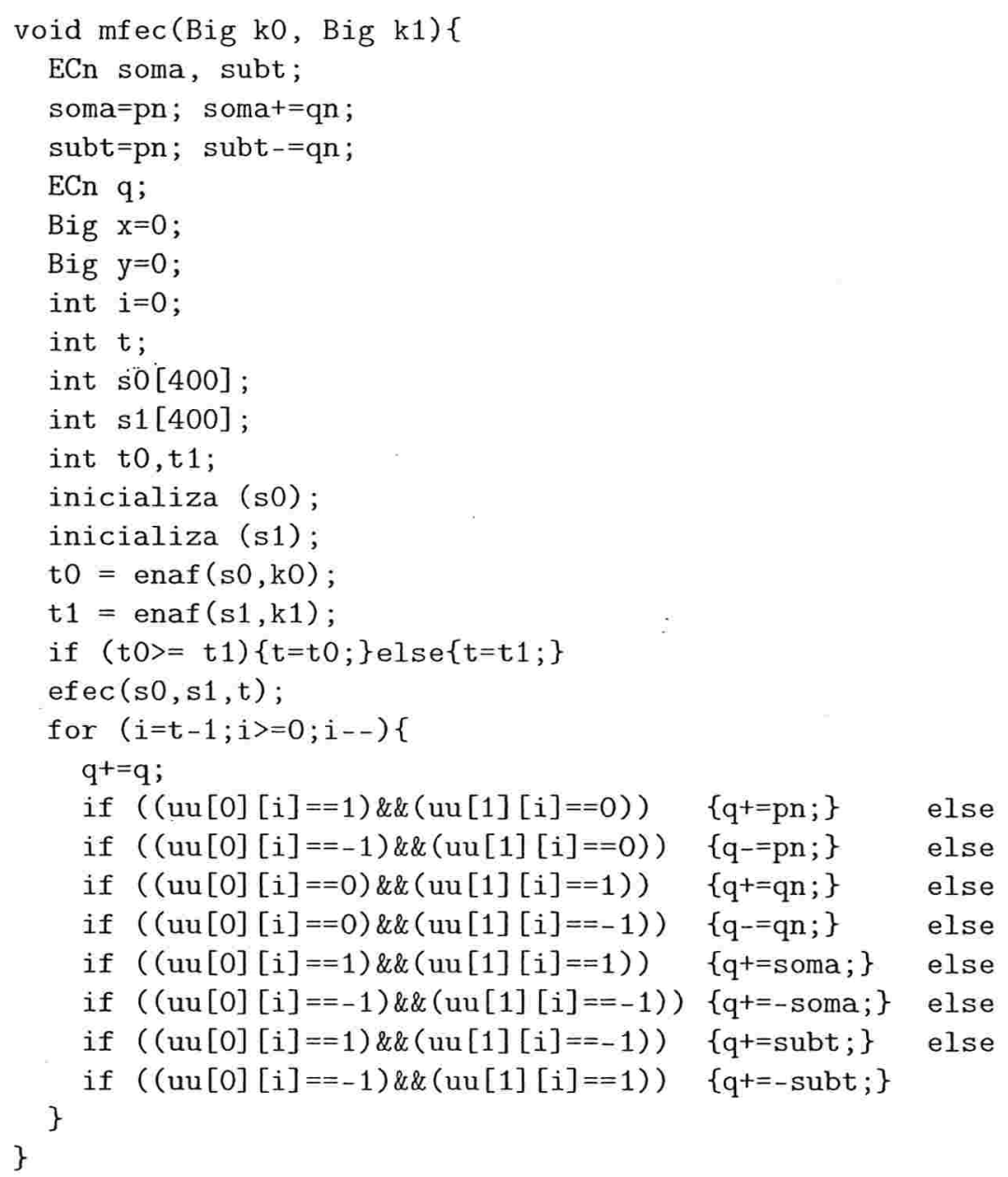

/*Multiplicação Simples: $\phi$-ádica de Ciet, Lange, Sica e Quisquater para curvas dos exemplos 10.1 e $10.2 \%$;

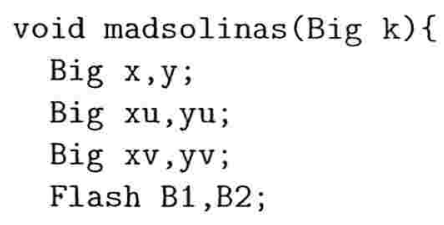


if $($ ipoc $==2)\{$

//Redução GLV de $K$ obtendo $K \equiv x_{u}+y_{u} \phi$

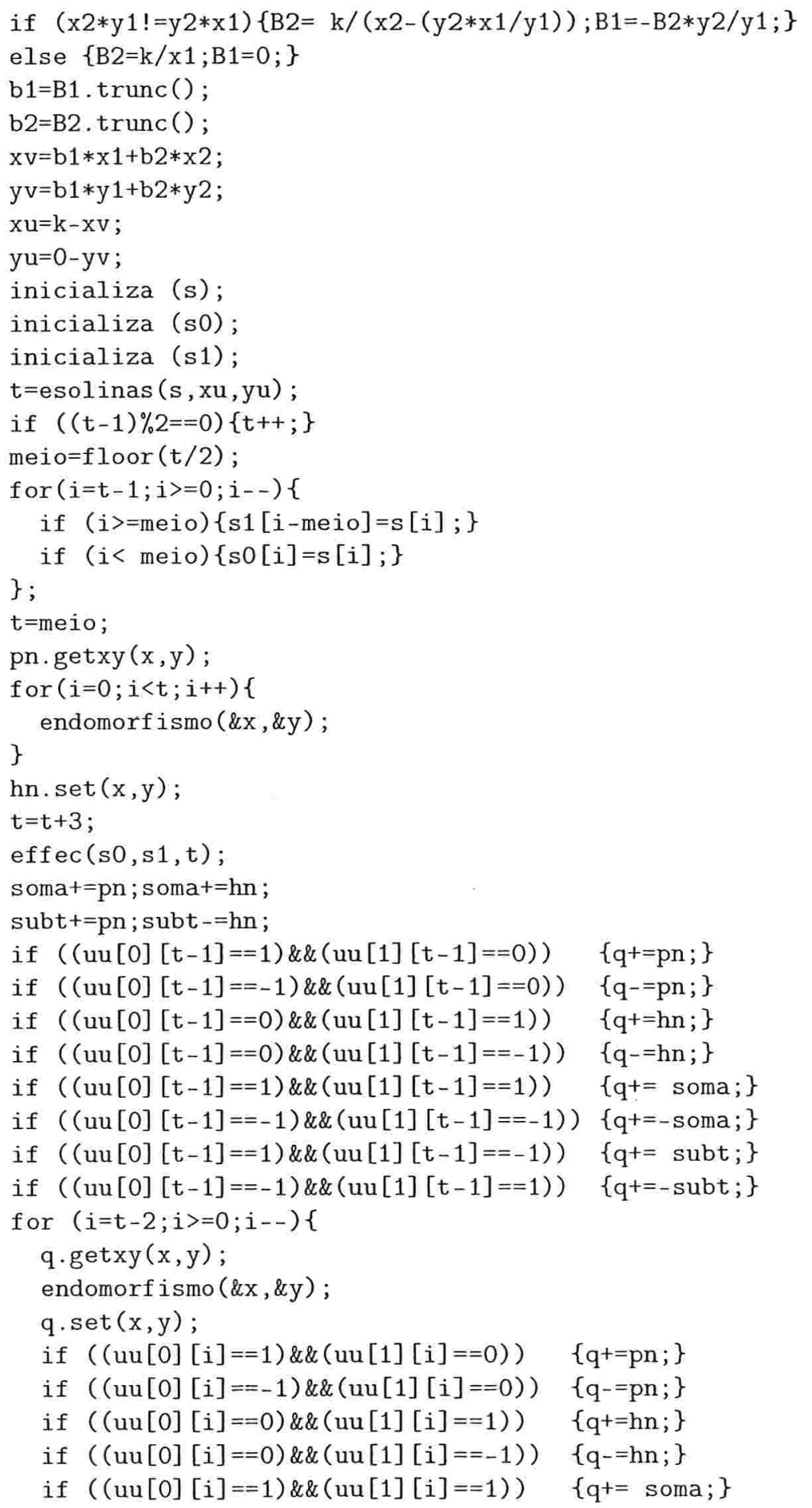




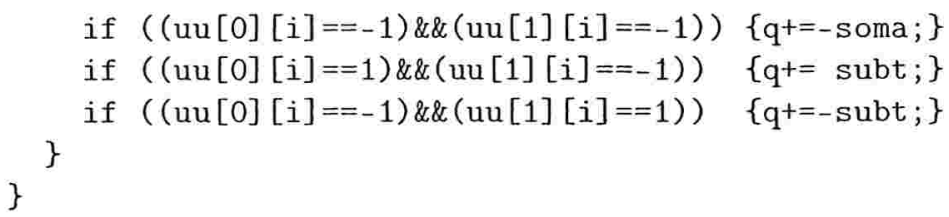




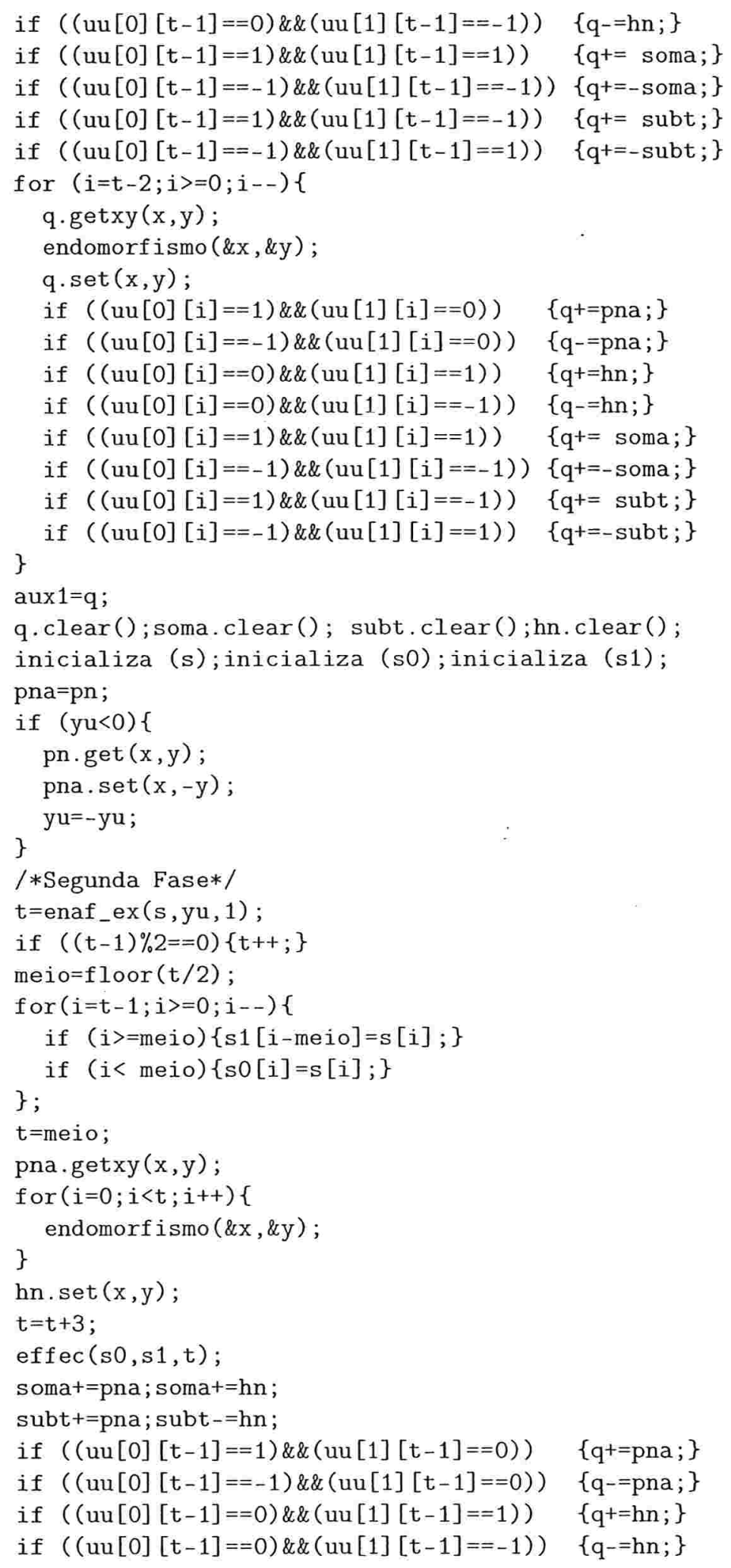




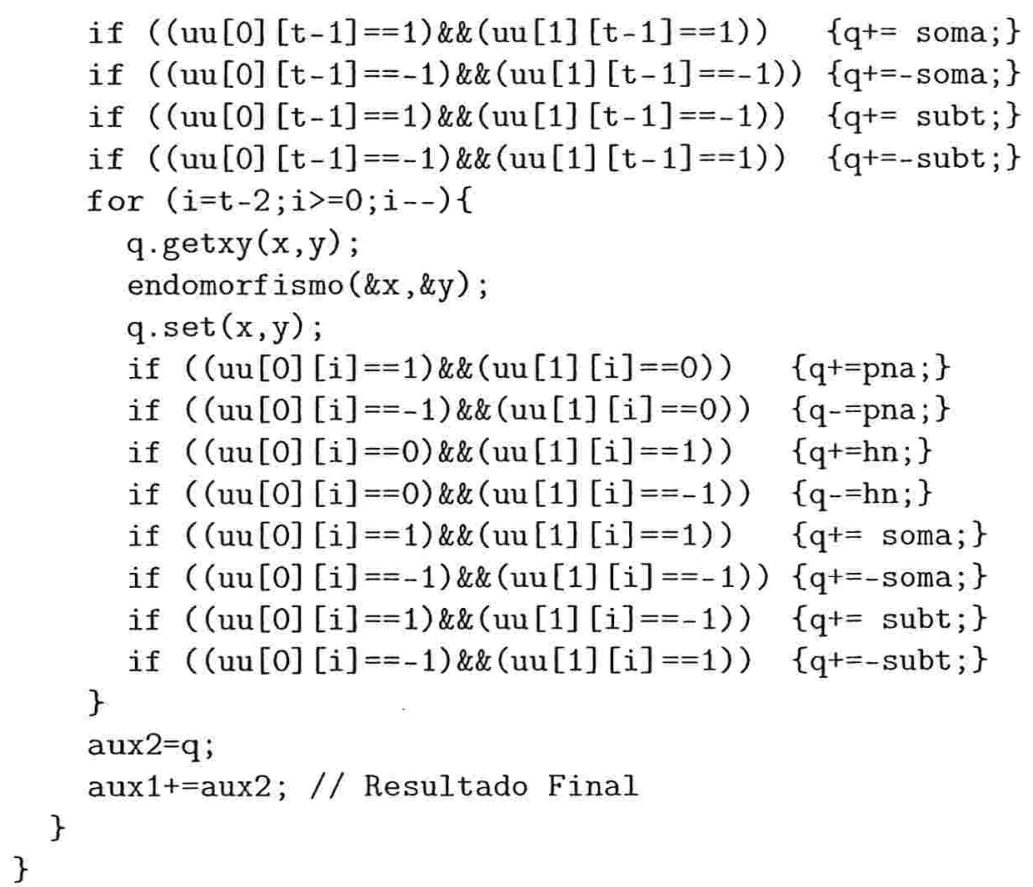

// Multiplicação Dupla: rápido de Shamir com $\phi$-FEC (algoritmo 10.3) nas curvas dos exemplos 10.1 e 10.2

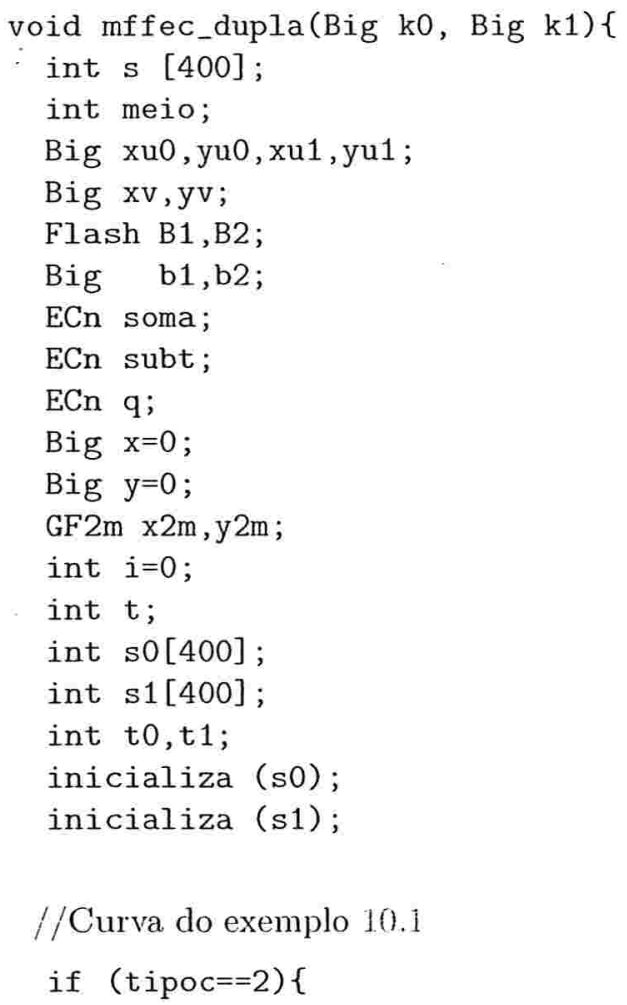




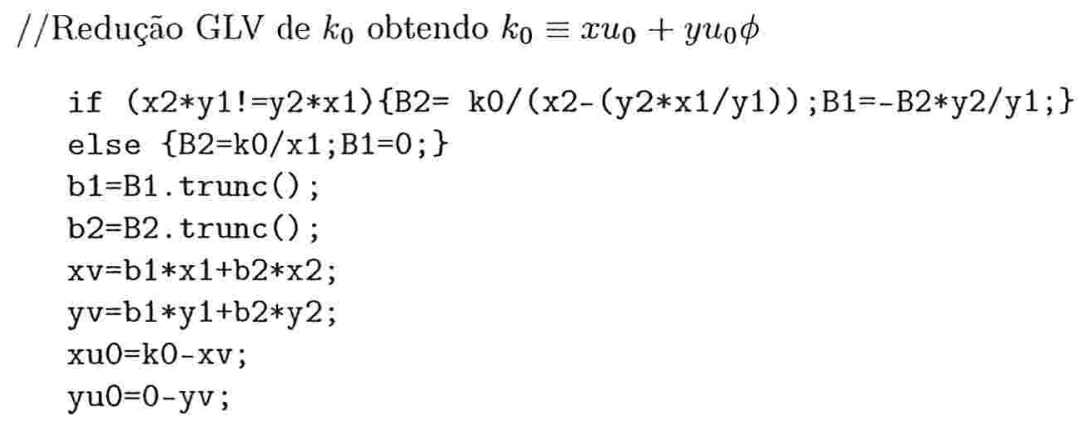

//Redução GLV de $k_{1}$ obtendo $k_{1} \equiv x u_{1}+y u_{1} \phi$

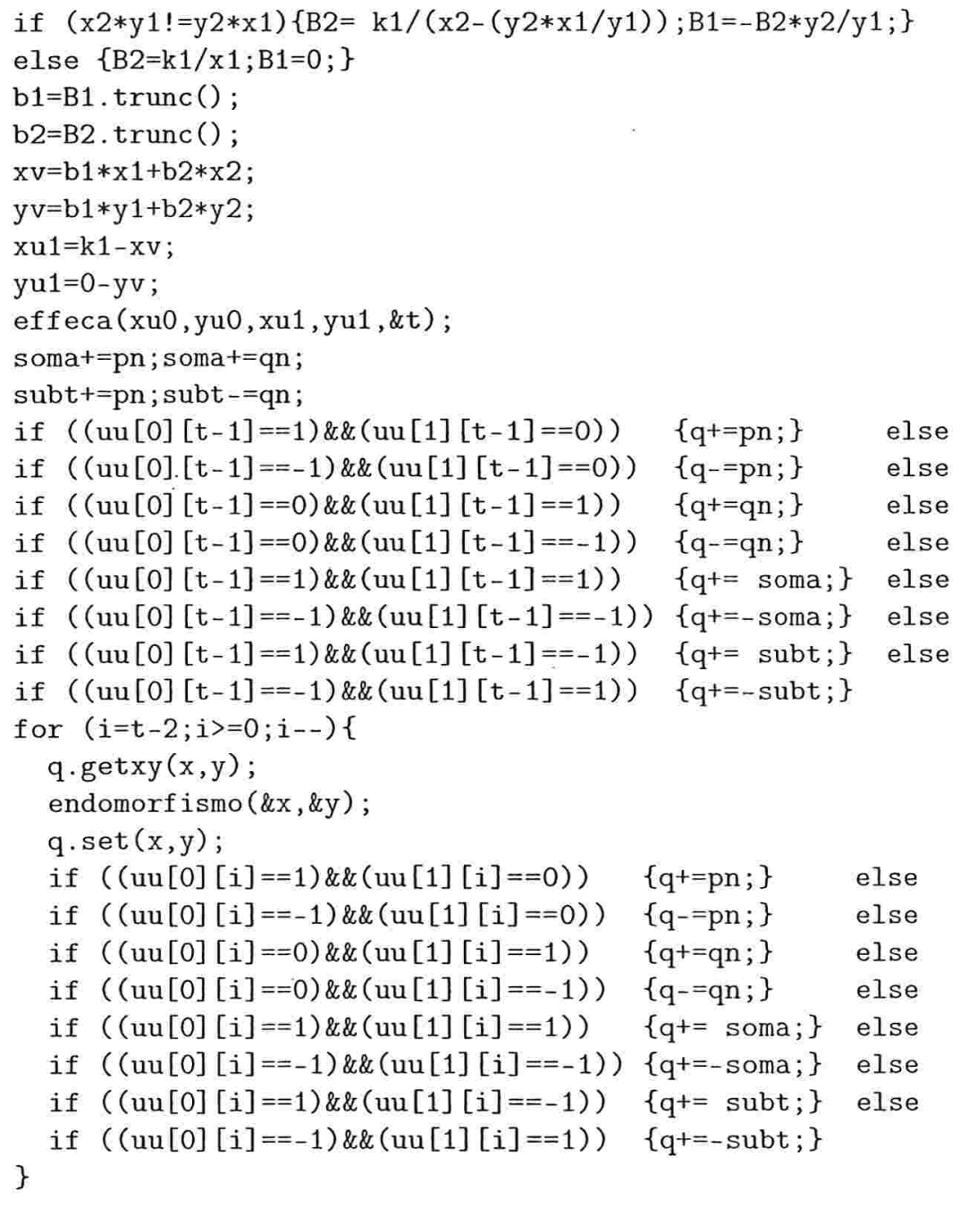

//Curva do exemplo 10.2 (ver seção10.6) 


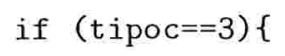




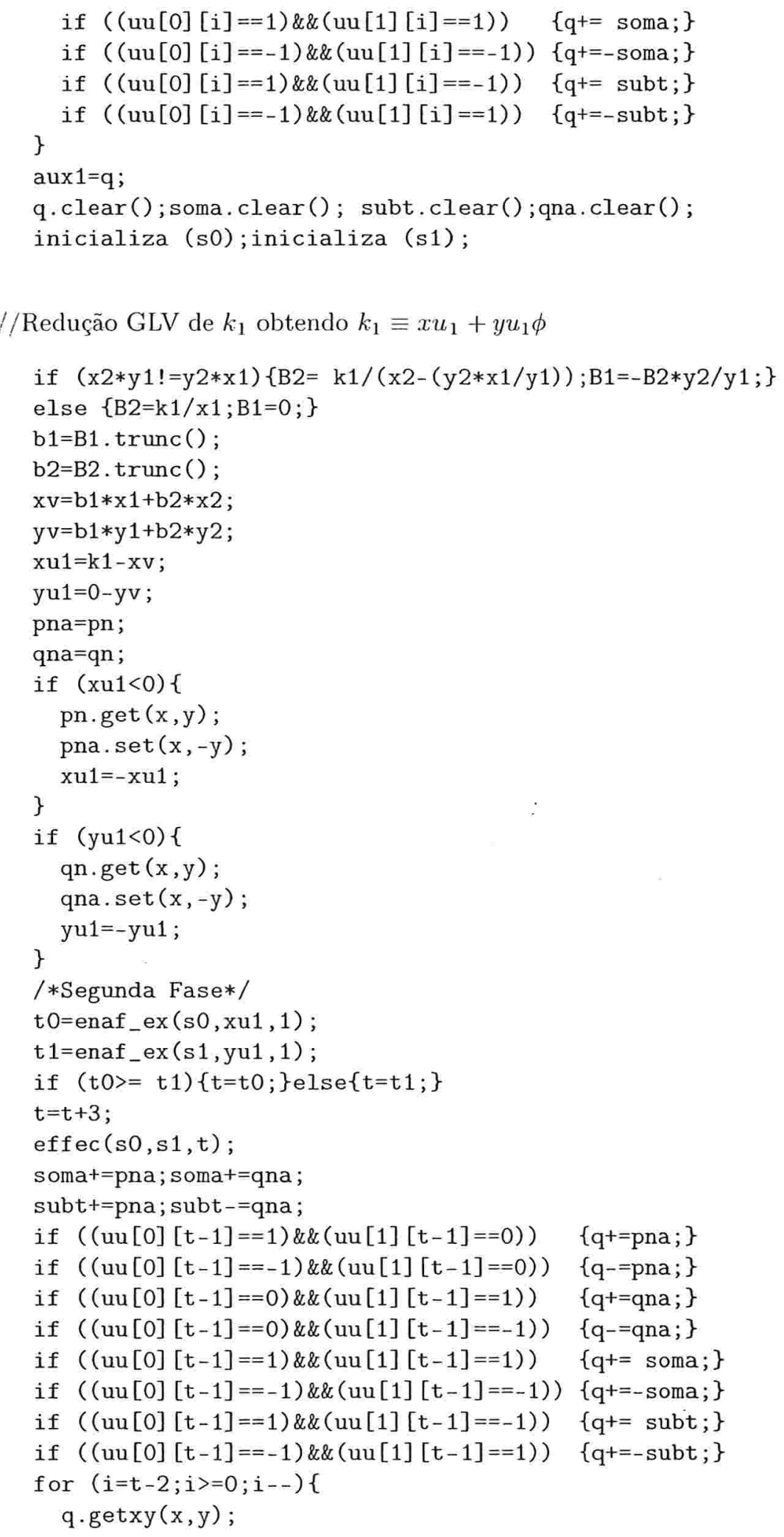




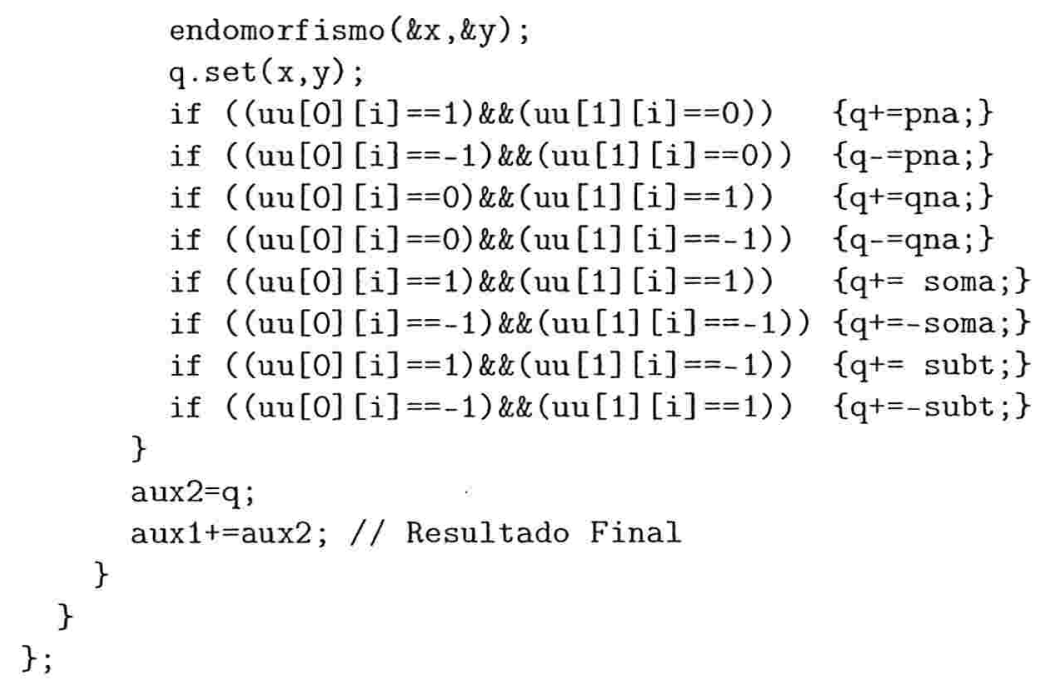




\section{Obtenção de parâmetros com o software MAGMA}

1. Verificando valor do traço de Frobenius de uma curva da seção 4.4.3: $E: y^{2}+x y=x^{3}+t$, onde $5 t \in \mathbb{F}_{4}$ satisfaz $t^{2}=t+1$

\section{Programa:}

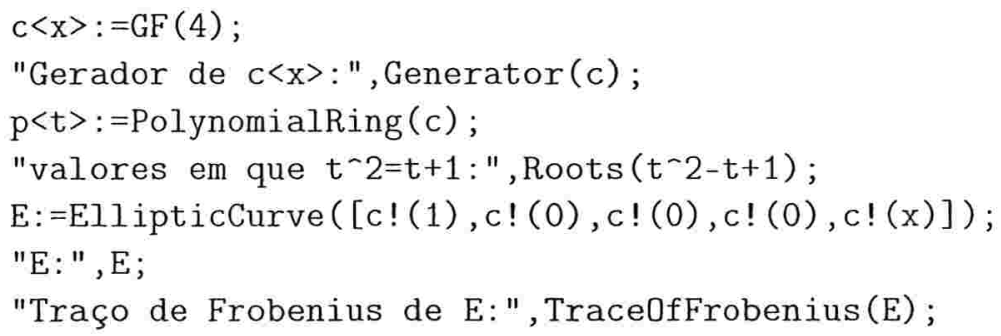

saída:

Gerador de $c<x>$ : $x$

valores em que $t^{\wedge} 2=t+1:\left[\langle x, 1\rangle,\left\langle x^{\wedge} 2,1\right\rangle\right]$

E: Elliptic Curve defined by $\mathrm{y}^{\wedge} 2+\mathrm{x} * \mathrm{y}=\mathrm{x}^{\wedge} 3+\mathrm{x}$ over GF(2^2)

Traço de Frobenius de E: 1

2. Verificando valor do traço de Frobenius de uma curva da seção 4.4.3: $E: y^{2}+x y=x^{3}+t x^{2}+t$, onde $t \in \mathbb{F}_{4}$ satisfaz $t^{2}=t+1$

\section{Programa:}

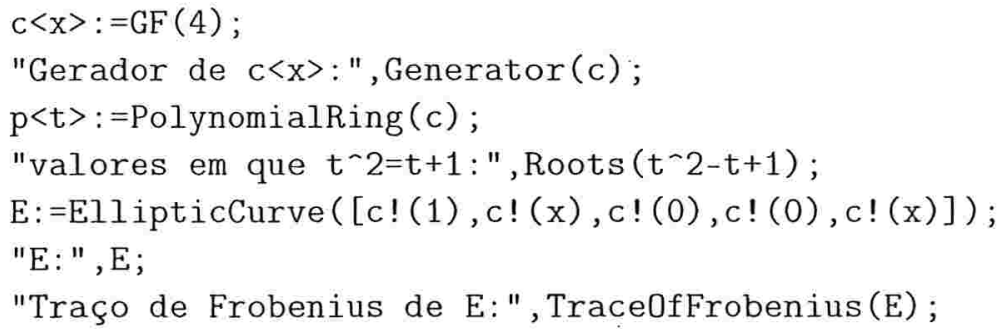

Saída:

Gerador de $c<x>$ : $x$ valores em que $t^{-} 2=t+1:\left[\langle x, 1\rangle,\left\langle x^{\wedge} 2,1\right\rangle\right]$

E: Elliptic Curve defined by $\mathrm{y}^{\wedge} 2+\mathrm{x} * \mathrm{y}=\mathrm{x}^{\wedge} 3+\mathrm{x} * \mathrm{x}^{\wedge} 2+\mathrm{x}$ over GF (2^2) Traço de Frobenius de E: -1 
3. Sabemos que $\phi(P)=\lambda P$ (seção 9.2), onde $\lambda$ é uma raiz do polinômio característico de $\phi$. Na curva do Exemplo 10.1 com $p=10007$, o algoritmo encontra $\lambda$.

\section{Programa:}

$$
\begin{aligned}
& \mathrm{p}:=10.007 \\
& \mathrm{~F}:=\mathrm{GF}(\mathrm{p}) \\
& \mathrm{p}<\mathrm{x}>:=\text { PolynomialRing }(\mathrm{F}) \\
& \mathrm{p}, \text { Roots }\left(\mathrm{x}^{-} 2-\mathrm{x}+2\right)
\end{aligned}
$$

\section{Saída:}

Univariate Polynomial Ring in $\mathrm{x}$ over GF(10007) $[\langle 4954,1\rangle,\langle 5054,1\rangle]$

4. Endomorfismo na curva do Exemplo $10.1 \mathrm{com} p=10007$.

\section{Programa}

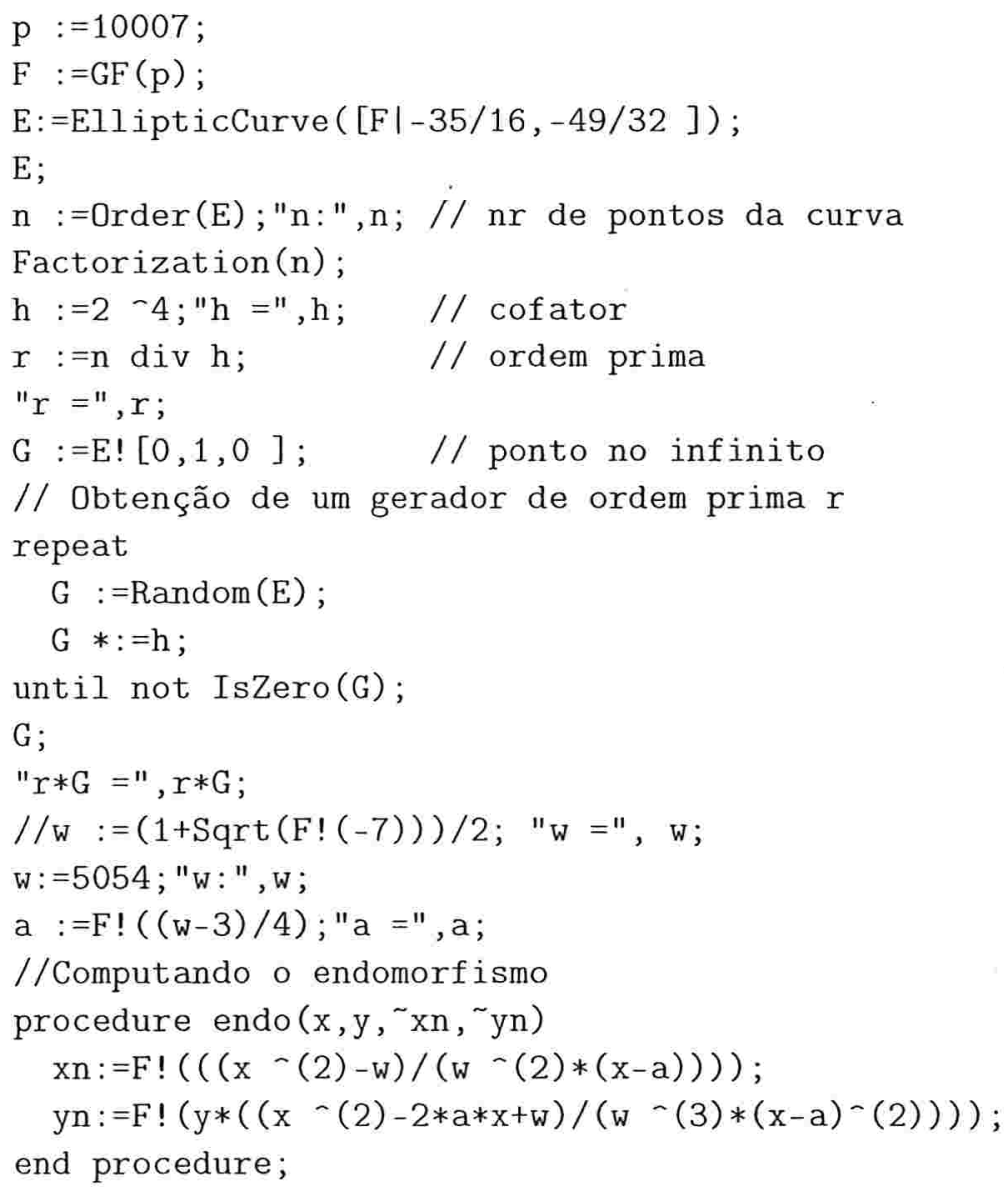




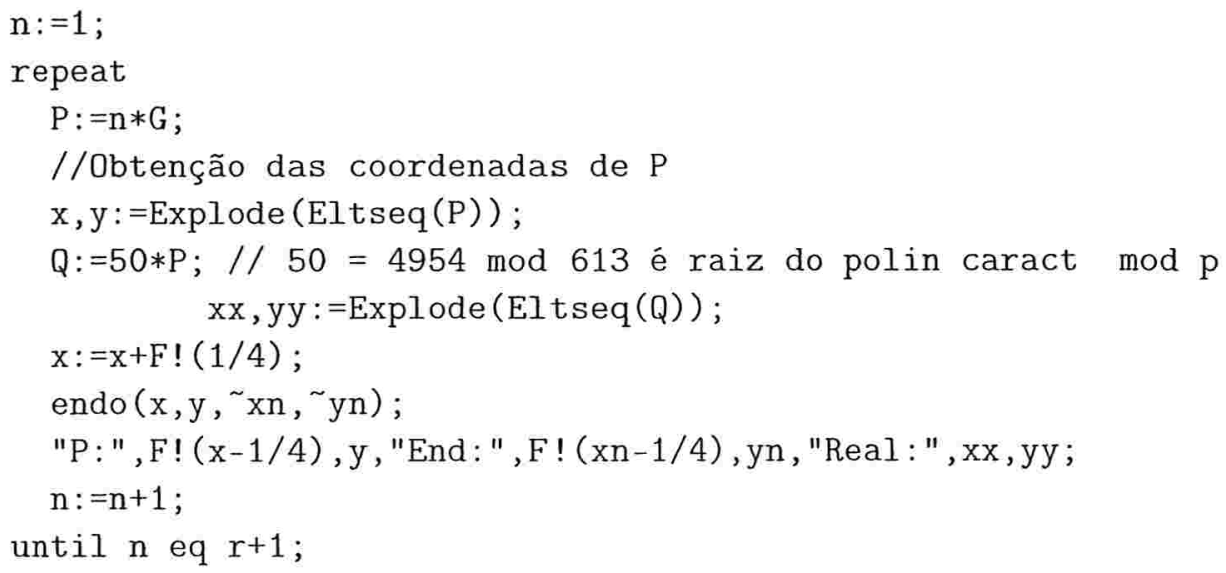

\section{Saída:}

Elliptic Curve defined by $\mathrm{y}^{\wedge} 2=\mathrm{x}^{\wedge} 3+3125 * \mathrm{x}+7191$ over $\mathrm{GF}(10007)$ $\mathrm{n}: 9808$

5. Endomorfismo na curva do Exemplo $10.2 \operatorname{com} p=443$.

\section{Programa:}

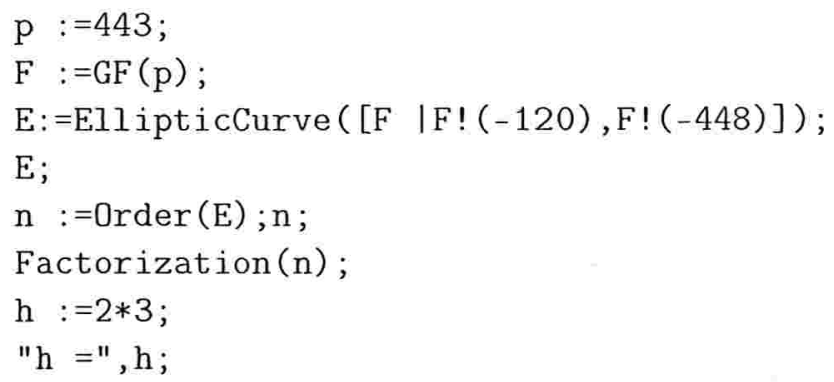




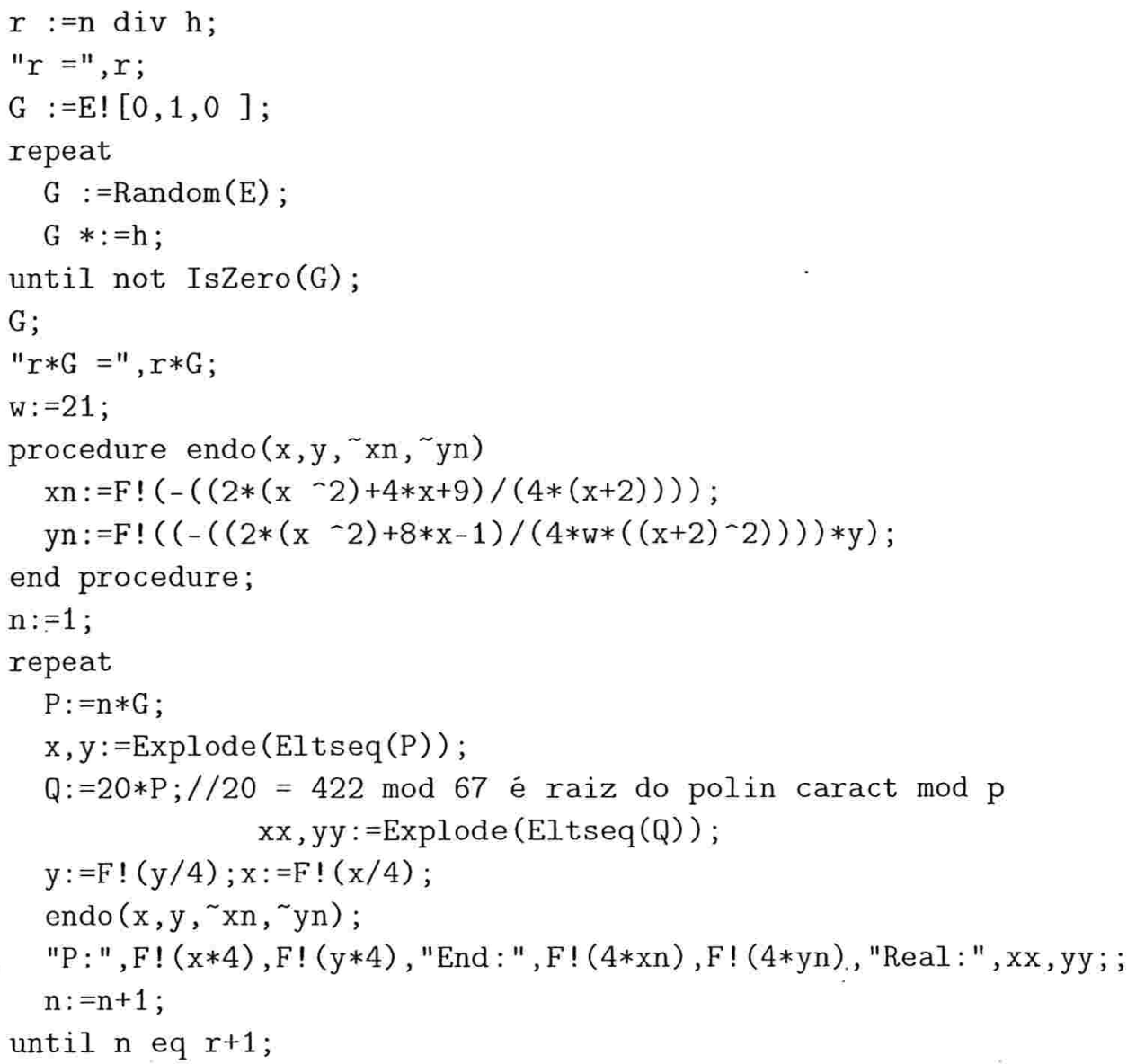

\section{Saída:}

Elliptic Curve defined by $\mathrm{y}^{\wedge} 2=\mathrm{x}^{-} 3+323 * \mathrm{x}+438$ over GF(443) 402

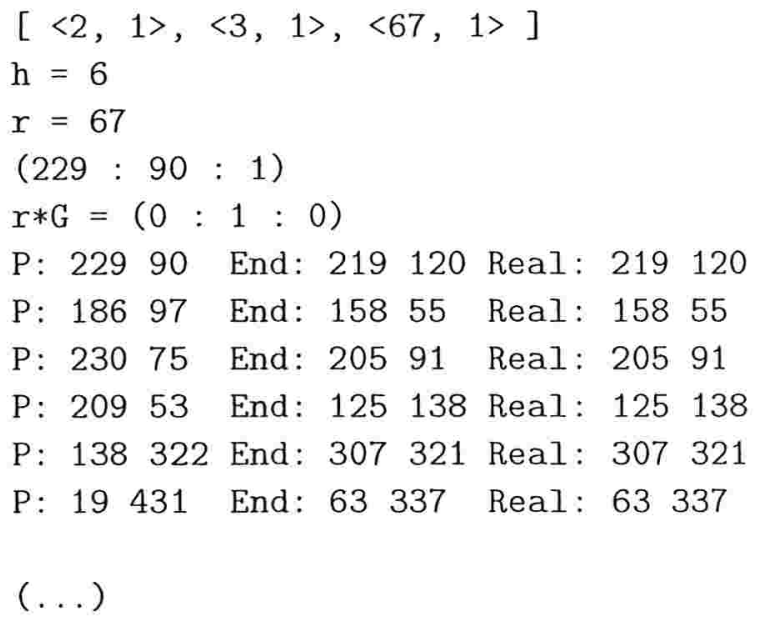


6. Nem todos os valores de $p$ são úteis. Na maioria das vezes o mapeamento endomórfico necessita ser aplicado mais de uma vez para que se obtenha o resultado correto, conforme é descrito abaixo (curva do exemplo 10.1)

\section{Programa:}

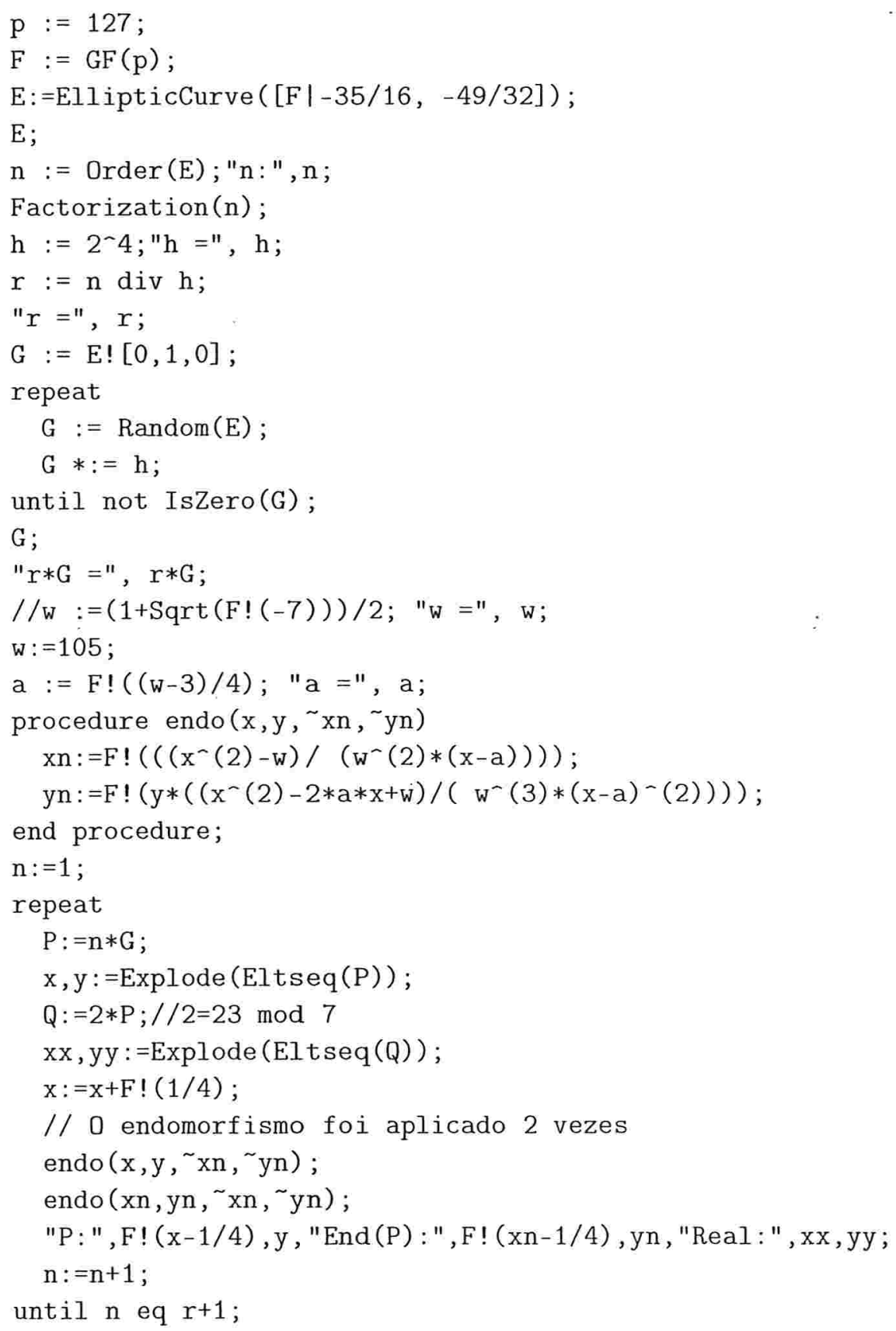

Saída: 


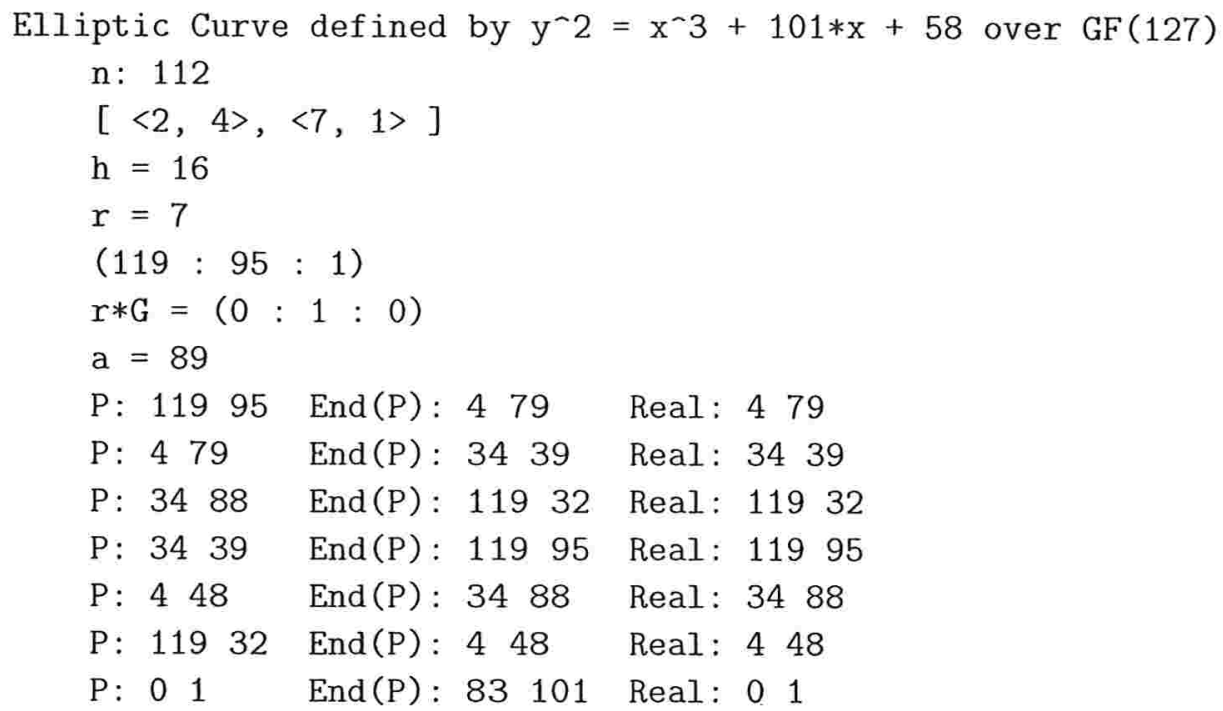




\section{Referências}

[1] Jurjen Bos and Matthijs Coster, Addition chain heuristics, Advances in Cryptology-Proceedings of Crypto'89, vol. 435, Springer-Verlag, 1990, pp. $400-407$.

[2] E. F. Brick, D. M. Gordon, K. S McCurley, and D. B. Wilson, Fast exponentiation with precomputation, Advances in Cryptology - Proceedings of Crypto'92, vol. 658, Springer-Verlag, 1992, pp. 200-207.

[3] Mathieu Ciet, Tanja Lange, Francesco Sica, and Jean-Jacques Quisquater, Improved algorithms for efficient arithmetic on elliptic curves using fast endomorfism, EUROCRYPT 2003, LNCS 2656 (2003), 388-400.

[4] Mathieu Ciet, Jean-Jacques Quisquater, and Francesco Sica, Analysis of the Gallant-Lambert-Vanstone method based on efficient endomorphism: elliptic and hyperelliptic curves, Proceedings of Selected Areas in Cryptography (SAC 2002), Lecture Notes in Computer Science, Springer, 2002 (To appear).

[5] Paul Erdös, Remarks on number theory III. on addition chains, Acta Arith., $77-81$.

[6] R. P. Gallant, J. L. Lambert, and S. A Vanstone, Faster point multiplication on elliptic curves with efficient endomorphism, Advances in CryptologyProceedings of Crypto 2001, vol. 2139 of Lecture Notes in Computer Science (J. Kilian, ed.), Springer, 2001, pp. 190-200.

[7] D. Gordon, A survey of fast exponentiation methods, Journal of Algorithms 27 (1998), 129-146.

[8] Darrel Hankerson, Alfred J. Menezes, and Scott Vanstone, Guide to elliptic curve cryptography, Springer.

[9] Donald E. Knuth, Seminumerical algorithms, 2nd ed., The art of computer programming, vol. 2, Addison-Wesley, Massachusetts, 1981.

$[10]$ N. Koblitz, CM Curves with good cryptographic properties, Advances in Cryptology-Crypto'91 (1992), 279-278.

[11] MAGMA, Interface Web do software MAGMA

(http://magna.maths .usyd, edu. au/calc)

e seu manual

(http://magna.maths .usyd edu. au/magma/htminelp/MAGMA.htm).

[12] Willi Meier and Othmar Staffelbach, Efficient multiplication on certain non-supersingular elliptic curves, Advances in Cryptology-Proceedings of Crypto'92, vol. 740, Springer Verlag, 1993, pp. 333-344.

[13] A. Menezes, T. Okamoto, and S. A. Vanstone, Reducing elliptic curve logarithms to logarithms in a finite field, Proceedings of the $23^{\text {rd }}$ ACM Symp. Theory of Computing, 1991.

[14] A. Menezes, P. van Oorschot, and S. A. Vanstone, Handbook of Applied Cryptography, CRC Press, 1996.

[15] A. Menezes and S. A. Vanstone, Elliptic curve cryptosystems and their implementation (to appear in J. Cryptology).

[16] MIRACL, Biblioteca $C / C++$ MIRACL, (ht.tp://indigo.ie/vrsscott/).

[17] F. Morain and J. Olivos, Speeding up the computations on an elliptic curve using addition-subtraction chains, vol. 24, 1990, pp. 531-544. 
[18] V. Müller, Fast multiplication on elliptic curves over small fields of characteristic two, Journal of Cryptology 1 (1998), 219-234.

[19] R. C. Mullin, I M. Onyszchuk, S. A. Vanstone, and R. M Wilson, Optimal normal bases in GF $\left(2^{n}\right)$, Discrete Appl. Math. 22 (1998/99), 149-161.

[20] Niger P. Smart, Elliptic curves over small fields of odd characteristics, Network System Department. HP Laboratories Bristo. HPL-09-126, October, 1997.

[21] J. A. Solinas, An improved algorithm for arithmetic on a family of elliptic curves, Advances in Cryptology-Crypto'97 (1997), 357-371.

[22] _ Low-weight binary representation for pairs of integers, Technical Report CORR 2001-41, CACR, 2001, available at

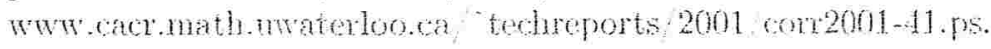

$[23] \ldots$, Efficient Arithmetic on Koblitz curves, Designs Codes and Cryptography 19 (2000), 195-249.

[24] _ Improved Algorithms for Arithmetic on Anomalous Binary curves, National Security Agency, Centre for Applied Cryptography Research.

[25] Routo Terada, Segurança de dados-criptografia em redes de computador, Edgard Blücher, 2001.

[26] A. C. Yao, On the evaluation of powers, SIAM J. Comput. 5 (1976), 100-103.

[27] WAP WTLS, Wireless Aplication Protocol Wireless Transport Layer Security Specification, Wireless Application Protocol Forum, February 1999, drafts available at:

Lit1), www.waponume.

[28] Onur Acíiçmez, Çetin Kaya Koç, and Jean-Pierre Seifert, On the Power of Simple Branch Prediction Analysis, CRYPTO 2006 (2006). 